Les infrastructures sont partout. Comme leur nom lindique, elles supportent nos vies quotidiennes, ce sont des ponts, des aéroports, des réseaux d'eau ou d'électricité, mais aussi des câbles sous-marins et des fermes de serveurs informatiques. Alors que l'on constate, en général, un clivage prononcé entre les chercheurs qui s'intéressent aux nouvelles infrastructures (autour du numérique) et les « fidèles » des infrastructures liées de prime abord à la première et la seconde révolution industrielle, les auteurs de cet ouvrage s'inscrivent indistinctement dans les deux groupes. Certains d'entre eux s'essaient même à explorer ce qu'il advient des infrastructures classiques à l'ère du numérique. Ce rapprochement permet d' approfondir ce qu'il y a de commun dans des infrastructures variées et de discuter de la pertinence de l'extension de la notion à d'autres domaines. Il permet également de souligner certaines évolutions partagées en particulier autour de l'affirmation de l'individu et de l'émergence d'une dimension politique jusque-là souvent enterrée, comme le sont bien des réseaux d'infrastructures.

Konstantinos Chatzis est chercheur à l'IFSTTAR affecté au LATTS (CNRS/UPEM/ENPC) et enseigne à l'École des Ponts ParisTech. Ses travaux portent sur la figure de l'ingénieur en France, en Grèce et auX États-Unis auX XIXe-XX|e siècles.

Gilles Jeannot est chercheur à l'École des Ponts ParisTech au LATTS (CNRS/UPEM/ENPC). Ses travaux portent sur le service public et la fonction publique.

Valérie November est directrice de recherche au CNRS et dirige le LATS (CNRS/UPEM/ENPC). Elle est spécialisée dans l'analyse des risques urbains, environnementaux et naturels, en particulier leurs effets sur le territoire.

Pascal Ughetto est professeur de sociologie à l'université Paris-Est Marne-la-Vallée, membre du LATTS (CNRS/UPEM/ENPC). Ses recherches portent sur les transformations du travail en lien avec les évolutions des stratégies, des outils de gestion et des dispositifs d'organisation. 




\section{Les métamorphoses des infrastructures, entre béton et numérique}

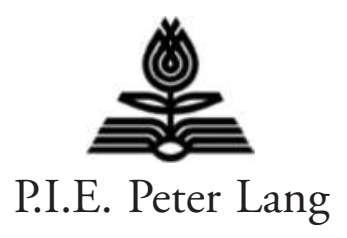

Bruxelles $\cdot$ Bern $\cdot$ Berlin $\cdot$ Frankfurt am Main $\cdot$ New York $\cdot$ Oxford $\cdot$ Wien 

Konstantinos Chatzis, Gilles Jeannot, Valérie November, Pascal Ughetto (dir.)

\section{Les métamorphoses des infrastructures, entre béton et numérique}


Cet ouvrage a été conçu et réalisé comme un projet collectif au sein du Laboratoire Techniques, Territoires et Sociétés (LATTS), laboratoire de recherche commun au Centre national de la recherche scientifique (CNRS), à l'Université Paris-Est Marne-la-Vallée (UPEM) et à l'Ecole des Ponts ParisTech (ENPC).

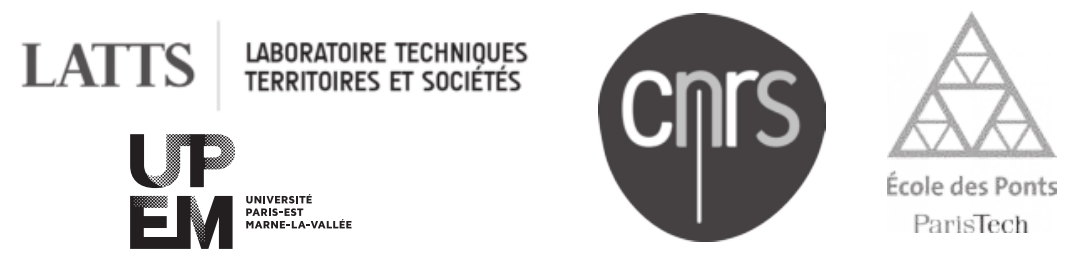

Illustration de couverture : Mélanie Pitteloud, film "Les gardiens du risque », 2012. Cette publication a fait l'objet d'une évaluation par les pairs.

Toute représentation ou reproduction intégrale ou partielle faite par quelque procédé que ce soit, sans le consentement de l'éditeur ou de ses ayants droit, est illicite. Tous droits réservés.

(C) P.I.E. PETER LANG s.a.

Éditions scientifiques internationales

Bruxelles, 2017

1 avenue Maurice, B-1050 Bruxelles, Belgique

www.peterlang.com ; brussels@peterlang.com

ISBN 978-2-8076-0599-2

ePDF 978-2-8076-0600-5

ePub 978-2-8076-0601-2

Mobi 978-2-8076-0602-9

DOI $10.3726 / \mathrm{b} 13091$

D/2017/5678/83

Information bibliographique publiée par "Die Deutsche Bibliothek »

"Die Deutsche Bibliothek " répertorie cette publication dans la "Deutsche Nationalbibliografie "; les données bibliographiques détaillées sont disponibles sur le site <http://dnb. ddb.de>. 


\section{Sommaire}

Introduction générale. Le nouveau monde des infrastructures.....19

Konstantinos Chatzis

INFRASTRUCTURE ET IMAGINAIRE

Infrastructure et imaginaires.

Une lecture alternative du changement technique

Antoine Picon

L'agrandissement de la métropole par l'infrastructure.

L'aéroport XXL de Hong Kong....

Nathalie Roseau

Les architectures événementielles, des infrastructures de la ville ?......95

Sarra Kasri

Naissance d'une infrastructure

Elsa Vivant

INFRASTRUCTURES URBAINES : LE PUBLIC ET LE PRIVÉ

Conglomérats familiaux et infrastructures essentielles.

Le cas des Philippines.

Dominique Lorrain et Morgan Mouton

La firme d'infrastructures locale et le rééchelonnement.

Daniel Florentin, Pauline Gabillet et Catalina Duque Gomez

LA VILLE POST-RÉSEAU

Au-delà de la ville des réseaux 163

Olivier Coutard et Jonathan Rutherford

Politiques d'infrastructures en Afrique subsaharienne.

Le réseau est-il soluble dans la transition urbaine?

Sylvy Jaglin 
Infrastructures urbaines et transition bas-carbone.

Spatialiser les processus de diffusion d'innovations de niche :

le cas des réseaux de chaleur en Alberta

Aïda Nciri

\section{LES INFRASTRUCTURES DE GESTION}

Les assemblages d'outils de gestion comme infrastructures de connaissance.

Gilles Jeannot et Pascal Ughetto

Les plateformes d'innovation, de marque, de conseil.

Vers une infrastructure numérique de la relation-client ?

Émile Gayoso, Thomas Jammet et Nicolas Klein

Les infrastructures numériques comme problème professionnel

Denis Duplan et Pascal Ughetto

Plateformes et infrastructures virtuelles

Les plateformes, étude d'une infrastructure numérique

Patrice Flichy

La gestion de crise : une infrastructure à part entière ?

Valérie November et Servane Gueben-Venière

Le processus d'infrastructuralisation de la télémédecine et de la télésanté. Vers une endogénéisation des dispositifs dans les standards existants?

Alexandre Mathieu-Fritz

\section{INFRASTRUCTURES ET POLITIQUE}

Les communs et les infrastructures des villes

Gilles Jeannot

Infrastructures numériques et production d'environnements personnalisés

Dominique Cardon 


\section{Avant-propos}

Cet ouvrage est le fruit d'une mobilisation collective des membres d'une unité de recherche française, le Laboratoire Techniques, Territoires et Sociétés (LATTS) (Centre national de la recherche scientifique, École des Ponts ParisTech, Université Paris-Est Marnela-Vallée). À une exception près, tous les auteurs sont ou ont été membres de ce laboratoire. L'idée de ce projet éditorial singulier est née d'un rapprochement inattendu : les chercheurs travaillant sur le numérique découvraient dans leur domaine l'émergence du thème des infrastructures, classiquement abordé par les collègues de leur laboratoire s'intéressant depuis de longues années à l'activité des ingénieurs et aux réseaux urbains. Le projet a été lancé par Olivier Coutard, alors directeur, qui a porté deux séminaires résidentiels sur ce thème et qui a su entraîner l'ensemble des composantes du laboratoire. Ces échanges ont aussi permis d'étendre l'interrogation sur les infrastructures vers des domaines moins explorés : la gestion des risques, le travail et les organisations. Cet ouvrage relie également de fait les deux revues soutenues par le LATTS : Réseaux, sur les enjeux du numérique, et Flux, sur les dimensions matérielles de l'urbain. Il permet de témoigner du renouvellement des thèmes de recherche du LATTS au moment où ce laboratoire vient de fêter ses trente ans. Les éditeurs de l'ouvrage tiennent à remercier Olivier Coutard, l'ensemble des chercheurs qui ont accepté de se plier à l'exercice, l'équipe administrative qui a participé à l'organisation des séminaires résidentiels et Aurélie Bur qui a assuré le suivi éditorial du projet, ainsi que les établissements scientifiques dont le LATTS relève et qui ont contribué au soutien à l'édition. 



\section{Les auteurs}

Cet ouvrage a été conçu et réalisé comme un projet collectif au sein du Laboratoire Techniques, Territoires et Sociétés (LATTS) ; tous les auteurs du recueil, à l'exception de Denis Duplan, co-auteur d'un des chapitres, sont ou ont été membres de ce centre de recherche commun au Centre national de la recherche scientifique (CNRS), à l'Université Paris-Est Marne-la-Vallée (UPEM) et à l'École des Ponts ParisTech (ENPC).

Dominique Cardon est professeur de sociologie à Sciences Po (Paris) où il dirige le médialab de Sciences Po. Ses travaux portent sur les usages d'Internet et les transformations de l'espace public numérique. Il étudie, en particulier, les algorithmes permettant d'organiser l'information sur le Web. Il a publié récemment $\grave{A}$ quoi rêvent les algorithmes. Nos vies à l'heure des big data (2015) et, avec Jean-Philippe Heurtin, Chorégraphier la générosité. Le Téléthon, le don, la critique (2016).

Konstantinos Chatzis est chercheur à l'IFSTTAR et enseigne à l'ENPC. Membre correspondant de l'International Academy of the History of Science, il a été chercheur invité au Georgia Tech (20102011) et à UCLA (2011-2012). Ses travaux portent sur la figure de l'ingénieur en France, en Grèce et aux États-Unis aux $\mathrm{XIX}^{\mathrm{e}}-\mathrm{XXI}^{\mathrm{e}}$ siècles. Éditeur associé de la revue Engineering Studies, auteur de plus de 130 publications, il est sur le point d'achever, pour le MIT Press, une histoire de la modélisation des déplacements urbains aux États-Unis de l'entre-deux-guerres à nos jours et prépare une biographie de l'ingénieur des ponts et chaussées Jules Dupuit (1804-1866).

Olivier Coutard est directeur de recherches au CNRS. Ses recherches portent sur les enjeux sociaux, spatiaux et environnementaux de la régulation des services urbains. Il travaille actuellement sur les politiques énergétiques urbaines et les enjeux urbains des transitions énergétiques ainsi que sur les transformations de l'organisation des services urbains ; il s'intéresse en particulier à la remise en cause des grands systèmes 
techniques, au développement de configurations sociotechniques alternatives et aux enjeux politiques de la "ville post-réseaux ». Parmi ses publications : (co-dirigé avec J. Rutherford) Beyond the networked city. Infrastructure reconfigurations and urban change in the North and South (2016) ; "Urban Energy Transitions: Places, Processes and Politics of Socio-Technical Change " (numéro co-dirigé avec J. Rutherford), Urban Studies (2014) ; (co-dirigé avec J.-P. Lévy) Écologies urbaines, Paris, Economica-Anthropos (2010).

Denis Duplan est informaticien et sociologue des organisations. Il intervient en tant que consultant (SOCIOPOLE) en entreprise sur des problématiques de conditions de travail.

Catalina Duque Gomez est urbaniste. Dans le cadre de ses études doctorales, elle a mené des recherches sur la gouvernance urbaine à travers les modèles de gestion et de production des services en réseau, avec un intérêt particulier sur le service d'électricité. Ses principaux terrains d'étude ont été Medellín (Colombie) et Nairobi (Kenya). Parmi ses publications : (avec S. Jaglin) "When urban modernisation entails service delivery co-production: a glance from Medellin ", Urban Research \& Practice (2016) ; (avec D. Florentin et P. Gabillet) «Larger, diversified and courted: the new triad of local firms of infrastructure in transition? ", Network Industries Quarterly (2015) ; "L'accès pérenne à l'électricité à Nairobi. Diversité d'expériences et tensions sur le réseau ", dans Zélem M.-C., Beslay C. (éds), Sociologie de l'énergie : gouvernance et pratiques sociales (2015).

Patrice Flichy est professeur émérite de sociologie à l'UPEM, directeur de la revue Réseaux. Il travaille principalement sur la sociologie d'Internet et des technologies numériques. Il a notamment publié : L'imaginaire d'Internet (2001) ; Le sacre de l'amateur (2010) ; "Rendre visible l'information. Une analyse socio-technique du traitement des données ", Réseaux (2013) ; Les nouvelles frontières du travail à l'ère numérique (2017).

Daniel Florentin est maître-assistant à l'École des Mines de Paris, au sein de l'Institut Supérieur d'Ingénierie et de Gestion de l'Environnement, PSL Research University. Il mène des recherches sur les changements dans les modes de fonctionnement des réseaux techniques urbains et sur les questions de maintenance et de gestion patrimoniale des réseaux d'eau 
et d'assainissement. Il a notamment publié : «La vulnérabilité des objets lents : les réseaux d'eau. Les enjeux des diminutions de consommation d'eau vus à travers un exemple allemand ", Les Annales de la recherche urbaine (2015); " La grande transformation infrastructurelle : les réseaux techniques face à la transformation post-socialiste. Les cas de l'eau et du chauffage urbain dans l'Est de l'Allemagne ", Revue géographique de l'Est (2016).

Pauline Gabillet est docteure en aménagement de l'espace et urbanisme (Les entreprises locales de distribution à Grenoble et Metz : des outils de gouvernement énergétique urbain partiellement appropriés, Université Paris-Est, 2015). Ses recherches portent sur les politiques énergétiques territoriales et sur le rôle des opérateurs énergétiques de réseaux dans leur élaboration et leur mise en œuvre. Elle est l'auteure de l'article « Energy supply and urban planning projects: analysing tensions around district heating provision in a French eco-district ", Energy Policy (2014).

Émile Gayoso a eu un parcours universitaire pluridisciplinaire, au croisement des sciences physiques et des sciences humaines et sociales. C'est finalement en sociologie de l'innovation qu'il réalisera sa thèse, entre le LATTS et le laboratoire de sociologie d'Orange Labs (SENSE). Ses recherches actuelles portent principalement sur la sociologie du numérique : plateformes de co-innovation, mouvement de l'Open Access, essor des métamarchés, etc. Parmi ses articles, on pourra lire en particulier : "Les plateformes de co-innovation. Enjeux gestionnaires et marchands de la participation des individus à l'innovation ", Réseaux (2015).

Servane Gueben-Venière est géographe, post-doctorante au Labex Futurs Urbains. Elle a soutenu, en 2014, une thèse sur le rôle des ingénieurs dans la gestion des risques littoraux (Pays-Bas, Angleterre et France) et travaille depuis 2015 sur les risques et les crises survenant en Île-de-France, au sein du programme de recherche dirigé par Valérie November et monté en partenariat avec la Préfecture de Police. Plus précisément, elle s'intéresse aux effets dominos et aux modes de représentations cartographiques utilisés en situation de crise. Parmi ses publications : (avec F. Sépot) «Sequana, grand échiquier des effets dominos ? ", dans November V., Créton-Cazanave L. (dir.), La gestion de crise à l'épreuve de l'exercice EU, Sequana 2016 (2017) ; " How do civil 
engineers see the coast they manage? A comparative approach between the Netherlands, England and France ", Coastal Management (2016) ; "How can mental maps, applied to the coast environment, help in collecting and analyzing spatial representations ", EchoGéo (2011).

Sylvy Jaglin est professeur à l'École d'Urbanisme de Paris à l'UPEM. Ses recherches portent sur les infrastructures urbaines en Afrique subsaharienne et interrogent les processus sociotechniques d'évolution des services en réseau. Ses travaux récents s'intéressent aux systèmes énergétiques. Elle a notamment publié: «Métropoles des pays émergents : des acteurs de la transition énergétique ? Leçons du Cap (Afrique du Sud) ", Géographie, Économie, Société (2017) ; (avec A. Dubresson) Eskom. Electricity and technopolitics in South Africa (2016).

Thomas Jammet a effectué une thèse de sociologie (Université Paris-Est, 2016) consacrée à la communication des entreprises et des organisations sur le Web 2.0, au prisme de l'activité de community management. Ses travaux portent sur l'activité professionnelle d'animation de communautés en ligne, la relation client numérique et les phénomènes de communication politique numérique. Parmi ses publications : "Les habits neufs de la communication de marque sur le Web social. Le travail d'intermédiation des community managers ", Sociologies pratiques (2017) ; (avec D. Guidi) «Observer Les Observateurs. Du pluralisme médiatique au populisme anti-islam, analyse d'un site de réinformation suisse et de ses connexions ", Réseaux (2017).

Gilles Jeannot est sociologue. Ses recherches portent sur le travail des fonctionnaires, les évolutions du service public et des administrations, en particulier face aux outils de gestion. Il a récemment publié : (avec O. Coutard) Revenir au service public? (2015) ; (avec P. Bezes), "Autonomy and managerial reforms in Europe : let or make public managers manage ? ", Public Administration (2017).

Sarra Kasri est architecte. Après un master de recherche en architecture sur " Le système des concours en tant qu'expression de l'architecture officielle actuelle en Tunisie », elle a complété sa formation par un Master d'ingénierie dans le cadre du Projet européen Tempus. Actuellement, Sarra Kasri est doctorante au LATTS où elle mène une recherche sur les logiques d'interactions entre l'architecture en tant que matérialités signifiantes habitées et les risques. Enseignante à l'École Nationale 
Supérieure d'Architecture de Paris-Belleville et à celle de Val-de-Seine, elle intervient sur la thématique de l'architecture et des risques majeurs en prolongement de ses travaux de recherche.

Nicolas Klein est doctorant au LATTS. Ses recherches portent sur le travail, l'organisation, les groupes professionnels et l'activité commerciale. Parmi ses publications : (avec M. Benedetto-Meyer), "Entre pratique commerciale et travail réputationnel : l'invention d'une nouvelle forme de relation client ?", Terrains \& Travaux (2017) ; (avec M. BenedettoMeyer), «Du partage de connaissances au travail collaboratif : portées et limites des outils numériques ", Sociologies pratiques (2015).

Dominique Lorrain, directeur de recherche émérite au CNRS, est socio-économiste. Il travaille depuis le début des années 1970 sur l'action publique urbaine. Parmi ses derniers travaux : (dir.) Métropoles en Méditerranée. Gouverner par les rentes, Paris, Presses de Sciences Po (2017) ; (co-édition avec F. Poupeau) Water regimes. Beyond the public and private sector debate (2016) ; (éd.) Governing megacities in emerging countries (2014).

Alexandre Mathieu-Fritz est professeur de sociologie à l'UPEM. Sociologue du travail, des organisations et des groupes professionnels, il développe des travaux se situant à la croisée des sociologies de l'activité, des TIC et de l'innovation, et se consacre à l'étude des mondes de la télésanté et de la télémédecine. Il a publié notamment : (avec L. Esterle), «Les transformations des pratiques professionnelles lors des téléconsultations médicales. Coopération interprofessionnelle et délégation des tâches ", Revue française de sociologie (2013) ; "Les médecins et le dossier santé informatisé communiquant. Analyse d'une expérimentation du dossier médical personnel (DMP) ", Réseaux (2013) ; "Teleconsultation in geriatrics: impact on professional practice ", International Journal of Medical Informatics (2013).

Morgan Mouton vient de soutenir (Université Paris-Est, 2017) une thèse qui porte sur la relation entre service électrique et changement urbain dans le Grand Manille (Philippines). Ses travaux, qui s'inscrivent dans les études urbaines et les Science and Technology Studies, interrogent les dynamiques d'émergence dans les grandes métropoles du Sud. Plus récemment, il a commencé une recherche sur la notion de " qualité " dans la production de l'espace urbain dans les villes nord-américaines. 
Aïda Nciri est doctorante et conduit ses recherches entre le LATTS et le Département de Géographie à l'Université de Calgary. Sa thèse porte sur la construction sociale, politique et matérielle des transitions bas-carbone dans les villes. En interrogeant la résurgence des réseaux de chaleur en Île-de-France et l'échec de leur diffusion en Alberta (Canada), cette recherche comparative montre que la transition bas-carbone est un processus contesté, soutenu par des politiques publiques sélectives et influencé par les intérêts économiques d'acteurs dominants dans le secteur de l'énergie et de la fabrique urbaine.

Valérie November est directrice de recherche au CNRS et dirige le LATTS. Elle est spécialisée dans l'analyse des risques urbains, environnementaux et naturels, en particulier leurs effets sur le territoire. Depuis quelques années, elle travaille sur la gestion de crise, et dirige depuis septembre 2015 le programme de recherche Euridice, centré sur les dispositifs de gestion de crise en partenariat avec la Préfecture de Police. Elle a publié plusieurs ouvrages, dont : Les territoires $d u$ risque : le risque comme objet de réflexion géographique (2002) ; (avec Y. Leanza), Risk, disaster and crisis reduction: Mobilizing, collecting and sharing information (2015) ; (co-dirigé avec L. Creton-Cazanave), La gestion des crises à l'épreuve de l'exercice EU Sequana (2017).

Antoine Picon est ancien élève de l'École polytechnique et de l'ENPC, architecte DPLG et docteur en histoire. Il est actuellement professeur à l'ENPC et à Harvard University, et président de la Fondation Le Corbusier. Ses recherches portent sur l'histoire des techniques et de l'architecture du XVIII siècle à nos jours. Il est l'auteur de nombreuses publications dont une vingtaine d'ouvrages. Parmi ces derniers : L'invention de l'ingénieur moderne. L'École des Ponts et Chaussées, 17471851 (1992) ; Les Saint-Simoniens : raison, imaginaire, et utopie (2002); Digital culture in architecture: an introduction for the design professions (2010) ; Ornament: the politics of architecture and subjectivity, (2013) ; Smart cities: a spatialised intelligence (2015).

Nathalie Roseau, est Associate professor de l'Ecole des Ponts. Ancienne élève de l'Ecole Polytechnique et de l'ENPC, elle est architecte DPLG et docteur en urbanisme. Ses recherches portent sur les dynamiques contemporaines des métropoles, les représentations urbaines et la question de l'infrastructure. Publié en 2012, Aerocity. Quand l'avion 
fait la ville porte sur l'influence de la mobilité aérienne et de ses infrastructures sur la ville. Elle a depuis co-publié plusieurs programmes de recherche sur l'histoire de la culture aérienne - (avec M. ThébaudSorger) L'emprise du vol : de l'invention à la massification, histoire d'une culture moderne (2013) -, la gouvernance des grandes métropoles (De la ville à la métropole. Les défis de la gouvernance métropolitaine (2013)), et actuellement sur l'histoire de l'aménagement du Grand Paris (www. inventerlegrandparis.fr, 2017).

Jonathan Rutherford est chercheur à l'ENPC. Il travaille sur les infrastructures urbaines et les dimensions matérielles et politiques du changement sociotechnique. Il a récemment codirigé des numéros spéciaux des revues Urban Studies (2014) et Energy Policy (2015) sur les transitions énergétiques urbaines ainsi que l'ouvrage Beyond the networked city: Infrastructure reconfigurations and urban change in the North and South (2016).

Pascal Ughetto est professeur de sociologie à l'UPEM. Ses recherches s'intéressent aux transformations du travail et aux enjeux d'organisation dans de nombreux secteurs d'activité, des services et de l'industrie, dans le privé comme dans le public. Il a notamment publié Faire face aux exigences du travail contemporain (2007).

Elsa Vivant est enseignante-chercheuse en urbanisme à l'UPEM. Ses recherches actuelles portent sur les collaborations entre artistes, urbanistes et chercheurs. Elle a récemment publié, avec Nadia Arab et Burcu Ozdirlik, Expérimenter l'intervention artistique en urbanisme (2016). 



\title{
Introduction générale
}

\section{Le nouveau monde des infrastructures}

\author{
Konstantinos Chatzis
}

\section{Une profusion d'objets et de textes}

Depuis une quinzaine d'années au moins, le terme d'infrastructure semble connaître une ascension fulgurante au sein de plusieurs discours, qu'ils soient politiques, économiques ou médiatiques ${ }^{1}$. Cette prolifération, loin de se cantonner dans un univers purement intellectuel, est, en revanche, en prise directe avec des réalités matérielles observées à grande échelle ; les mots renvoient, en effet, à des réalisations massives, voire à des situations critiques, les infrastructures devenant soudainement synonymes de risques et de catastrophes. Particulièrement inclusifs, discours et réalités accueillent aussi bien les vielles infrastructures, tels les réseaux d'eau et d'assainissement ou les réseaux électriques, qui ont tant marqué de leur sceau la ville du XIX et du premier XX $X^{\mathrm{e}}$ siècle (Tarr et Dupuy, 1988 ; Dupuy, 1991), que les infrastructures informationnelles d'extraction plus récente, qui produisent et véhiculent toutes sortes de données "immatérielles ", par l'intermédiaire, précisons-le immédiatement, d'objets parfaitement matériels - pensons aux parcs de serveurs, forts consommateurs en énergie au demeurant. Le nouveau vient souvent, par ailleurs, au secours de l'ancien pour le rendre enfin smart (Royal Academy of Engineering, 2012), à l'instar de ces autoroutes urbaines équipées désormais d'une multitude de capteurs et de panneaux à messages variables, qui font circuler en temps réel de l'information sur l'état du trafic entre des centres de régulation et les conducteurs

Je souhaite remercier ses coéditeurs ainsi que le rapporteur anonyme, qui a expertisé ce recueil pour le compte de la maison d'édition, pour leurs commentaires et suggestions lors d'une version antérieure du chapitre. Il va de soi que je porte seul la responsabilité du texte final. 
dans l'objectif d'une exploitation maximale des potentialités du réseau existant (Chatterjee et McDonald, 2004)2 . Pièce intéressante à verser également au dossier, les infrastructures ne sont plus l'apanage d'un Premier Monde déjà riche et puissant. Imposant, de façon variable il est vrai, leur présence dans un nombre croissant de pays caractérisés par des trajectoires sociopolitiques et des niveaux économiques variés (Lorrain, 2011), elles parcourent, par ailleurs, toutes les échelles spatiales : locale, nationale, internationale (Brenner, 2004).

Cette ubiquité des infrastructures, dans les discours et dans les faits, ne risque pas d'être un phénomène passager, tant elle est portée par des forces à l'œuvre dans les sociétés contemporaines, entraînées aujourd'hui par les courants de la globalisation. Dans les parties du monde qui bénéficient déjà d'une prospérité économique pluriséculaire, l'âge des infrastructures déjà installées pose en termes urgents les questions de leur remplacement et de leur renouvellement, alors que dans le reste de la planète l'absence ou la rareté des infrastructures sur place appellent à leur développement. Posée avec une grande intensité dans tous les pays, anciennement et nouvellement industrialisés, la question du développement durable commande à son tour des politiques qui bousculent des trajectoires technologiques établies de longue date, dans les secteurs de l'énergie et des transports notamment, tout en suscitant de nouveaux développements infrastructurels. Ceux-ci sont soutenus par des puissants acteurs économiques, qui voient dans les infrastructures des assets rentables et peu risqués ou des nouvelles sources d'efficacité - pensons ici au recours de la part de grandes organisations à des systèmes d'information de plus en plus intégrés. Surfant sur la vague de la seconde mondialisation ${ }^{3}$, profitant à la fois de la pénurie financière des acteurs publics et des politiques pro-business d'un certain nombre

2 Un autre réseau traditionnel, celui des égouts, est également toujours davantage équipé aujourd'hui de capteurs transmettant en temps réel à des centres de contrôle et de pilotage des informations sur l'état du réseau et des flux qui y circulent. Voir, entre autres, le numéro spécial de la revue Urban Water Journal (2013). Sur les mutations organisationnelles du service de la Seine-Saint-Denis suite à l'introduction de la gestion automatisée du réseau d'assainissement du département éponyme, une première dans la France de l'époque, voir Chatzis (2000).

3 Pour la première vague, voir, par exemple, Marnot (2012). L'auteur consacre par ailleurs plusieurs développements (ch. 7, 8 et 9) aux infrastructures, celles de transport notamment, impliquées dans la première mondialisation. 
d'acteurs supranationaux puissants ${ }^{4}$, de grandes firmes font par ailleurs circuler de véritables modèles infrastructurels à travers le globe (Lorrain, 2011b ; Coutard et Rutherford 2016).

Face à des changements de cette ampleur, il aurait été vraiment étonnant que le monde académique restât silencieux. Depuis un certain temps, on enregistre, en effet, un flux continu de travaux où l'infrastructure est présente sous forme d'objet d'étude propre, mais aussi en tant que terrain au service d'une question particulière, comme armature conceptuelle et grille de lecture du réel enfin (Howe et al., 2016; Wegrich et al., 2017; Harvey et al., 2017). L'inflation notionnelle qui entoure le terme d'infrastructure témoigne à sa façon de l'ampleur de cette production scientifique. On parle ainsi du champ émergent d' « infrastructure studies »-dans le domaine de réseaux informationnels notamment (Edwards et al., 2009) -, on inscrit ses propres travaux dans le " new infrastructural turn »(Amin, 2014), on définit des agendas de recherche visant à l'étude systématique des infrastructures, on se forge des outils et des approches particulières pour les explorer (Star, 1999 ; Pollock et Williams, 2010). Des expressions plus ou moins élaborées conceptuellement comme " infrastructural inversion " (Bowker, 1994), " knowledge infrastructures » (Edwards et al., 2012), " infrastructural power » (Mann, 2012), " infrastructure state » Guldi, 2012), " critical infrastructures " " " pouvoirs des infrastructures " (Roseau, 2016), " infrastructures de la vie sociale " (Urry, 2005), " infrastructural event » (Bishop et Phillips, 2014), " infrastructuring aid " (Donovan, 2015), " revolutionary infrastructure » (Boyer, 2017), « infrastructural relation » (Harvey, 2012), « multinatural infrastructure » (Jensen, 2017), « infrastructural violence » (Ethnography, 2012), " infrastructural fetishism » (Namba, 2017), " urban infrastructures regimes " (Monstadt, 2009), " infrastructuring " (Le Dantec et DiSalvo, 2013), " infrastructuralization »(Chu, 2014 ; Day, 2014), « infrastructural lives » (Graham et McFarlane, 2015), « infrastructural semiosis » (Mackenzie, 2017) figurent dans des articles et des ouvrages

4 On parle souvent alors de « Washington consensus ». Sur ce dernier, voir en premier lieu, les réflexions de son père spirituel, John Williamson, à distance de plusieurs années après la première formulation dudit consensus en 1989 (Williamson, 2008).

5 Une revue dédiée à ce type d'infrastructure, The International Journal of Critical Infrastructures, a vu même le jour en 2004. 
appartenant à des horizons intellectuels et disciplinaires variés, alors que des réponses à la question frontale " qu'est-ce qu'une infrastructure ? " sont aussi tentées (Neuman, 2006). L’entrée "infrastructure », comme terrain ou comme armature conceptuelle, est régulièrement mobilisée pour penser sur de nouveaux frais aussi bien une série de questions qui ont fait déjà coulé beaucoup d'encre en sciences humaines - l'État en action (Rowland et Passoth, 2015 ; Chu, 2014 ; Harvey et Knox, 2015), les réalités " techno-politiques" (rapports entre politique et expertise technique) et "biopolitiques " (le gouvernement des populations et, plus généralement, de la vie $)^{6}$ - que des problèmes plus modestes et circonstanciés, comme la mise en place d'un système de bio-indicateurs dans le Sahel (Tousignant, 2013) ou les politiques de gestion hydrique du bassin versant autour du canal de Panama (Carse, 2012, 2014). Dans d'autres travaux, les infrastructures sont dépeintes comme les organes principaux du métabolisme de nos sociétés urbaines (Graham, 2010) et, à en croire certains chercheurs, les infrastructures et (notre) condition moderne sont prises, ni plus ni moins, dans des rapports de construction mutuelle (Graham et Marvin, 2001 ; Edwards, 2003 ; Larkin, 2013 ; Coutard et Rutherford, 2016).

\section{Un nouvel ouvrage sur les infrastructures aujourd'hui}

Le présent ouvrage s'inscrit dans cette double conjoncture, à la fois intellectuelle et factuelle, marquée par la prolifération de discours et de réalisations matérielles. Au-delà de la langue de publication adoptée ${ }^{8}$, notre entreprise éditoriale se singularise aussi au sein de la littérature

$6 \quad$ Voir rapidement le bel article de synthèse de Larkin (2013), qui contient une riche littérature sur l'usage de l'" infrastructure " dans plusieurs travaux anthropologiques récents. À la liste biblioraphique proposée par l'auteur, on peut ajouter l'ancien article de Michel Marié paru en 1984 (Marié, 1984).

7 Des auteurs comme Stephen Graham et Simon Marvin parlent plus particulièrement de "modern infrastructural ideal ", pour qualifier la place et le rôle (réel ou projeté) des réseaux techniques urbains dans la ville des temps modernes.

8 Rappelons que la littérature en matière d'infrastructures est essentiellement écrite en anglais. S'agissant de la langue de publication, signalons toutefois ici deux entreprises éditoriales françaises : l'ouvrage collectif intitulé Les temps des infrastructures (Prelorenzo et Rouillard, 2007) et le numéro en préparation dédié aux infrastructures de la revue Tracés (https://traces.hypotheses.org/1885, consulté le 15 septembre 2017). 
existante par une série de traits particuliers, parmi lesquels il faut citer, en premier lieu, la décision de traiter en un seul lieu plusieurs " générations » et types d'infrastructures, la volonté d'être aussi global (spatialement parlant) que possible en s'intéressant à une pluralité de contextes géographiques, la présence, enfin, comme élément unificateur, d'un ensemble de sensibilités et de vues générales partagées qui parcourent le recueil, collectivement construites par les contributeurs au fur et à mesure de leurs échanges et interactions en tant que membres, présents ou passés, du même centre de recherche.

Ainsi, du point de vue thématique, alors qu’on constate, en général, un clivage prononcé entre ceux qui s'intéressent davantage aux " nouvelles » infrastructures (autour du numérique) et les « fidèles » des infrastructures liées de prime abord à la première et la seconde révolution industrielle ${ }^{9}$, les auteurs de cet ouvrage se recrutent indistinctement dans les deux groupes mentionnés, et pour certains d'entre eux, ils essaient même d'explorer ce qu'il advient des infrastructures classiques à l'ère du numérique. Cet intérêt pour la " numérisation » des infrastructures classiques trouve son pendant dans une réflexion sur la question de l'" infrastructuralisation du numérique ", à savoir la transformation de dispositifs locaux et disparates de production et de gestion de données en des systèmes de plus en plus étendus, capables de capter, stocker, traiter et diffuser des masses d'informations et en opérant à des échelles tant spatiales que sociales inconnues jusqu'à présent. De même, la barrière intellectuelle qui sépare aussi souvent au sein du monde académique les villes du « Nord » des villes $\mathrm{du}$ "Sud » n'est pas ici de mise non plus : ainsi, Alberta côtoie Hong Kong et Manille alors que l'Europe figure à côté de l'Afrique subsaharienne et de l'Amérique latine. Ce pluralisme en matière d'espace trouve également son pendant temporel dans l'intérêt porté sur la longue durée, la seule qui permet d'établir les continuités qui peuvent caractériser le monde des infrastructures à travers le temps et de souligner en même temps les bifurcations opérées sous nos yeux et les originalités de la situation présente. Il faut noter aussi que, pour la plupart des auteurs du recueil, quoiqu'à des degrés divers, l'infrastructure fonctionne à la fois comme objet propre d'analyse - c'est le cas pour la majorité des études ici réunies et comme outil, sous forme de terrain empirique ou de grille d'analyse, au

9 Voir aussi le texte de présentation de la collection «Infrastructures » chez The MIT Press. 
service de questionnements et de problématiques variés, regroupés pour l'essentiel autour du thème général de l'" action organisée " développée par des (grandes) firmes privées et des organisations publiques ${ }^{10}$. Autre caractéristique de notre entreprise éditoriale enfin, son côté résolument pluridisciplinaire et "pluri-public ", ce qui ne va pas sans imposer aux auteurs une série de contraintes formelles et stylistiques : les contributions sont voulues ainsi courtes, certes rigoureuses dans l'administration de la preuve, mais sans l'apparat d'érudition qui convient à des articles destinés à une revue disciplinaire, en somme lisibles à la fois par l'honnête homme et par le chercheur non intéressé par les mille et un détails qui chargent les débats académiques au sein d'une discipline qui n'est pas la sienne.

Si l'ouvrage que voici est caractérisé, nous venons de le voir, par une pluralité de voix, une série de sensibilités communes et de traits partagés traversent, comme un écheveau de fils, parfois groupés, parfois de façon plus individuelle, les différentes contributions du recueil. Pour commencer, les auteurs du livre se sont finalement ralliés, non sans débats et échanges d'arguments et de contre-arguments, à une conception commune, somme toute assez classique des infrastructures et proche de celle qu'on trouve dans plusieurs travaux figurant dans la littérature spécialisée, conception qui met en avant à la fois la matérialité des infrastructures et leur capacité à affecter le "grand nombre " de façon plus ou moins pérenne ${ }^{11}$. Les infrastructures

10 Ainsi, pour donner un exemple, deux auteurs du recueil ont voulu se frotter à la littérature sur les infrastructures pour explorer le monde des outils de gestion dans les entreprises, ceux-ci n'étant pas pensés spontanément en termes d'infrastructure.

11 Pour des conceptions plus articulées, voir les contributions des Edwards, Star, Neuman, citées dans la bibliographie à la fin du chapitre, ainsi que le chapitre rédigé par Antoine Picon pour le présent ouvrage. Voici enfin un petit échantillon de conceptions récentes de l'infrastructure, assez proches de celle adoptée par les auteurs du recueil : « [...] infrastructures are extended material assemblages that generate effects and structure social relations, either through engineered (i.e. planned and purposefully crafted) or non-engineered (i.e. unplanned and emergent) activities " (Harvey et al., 2017b, p. 5) ; "Normally, one associates the term "infrastructure" with massive, durable works of material artifice: bridges, dams, highways, grids, to name a few. Even when such works are multiple and invisible (e.g. pipes, wiring), even when the term is used metaphorically, it still conjures images of material systems that are either singularly massive or near ubiquitous in their distributed networks " (Boyer, 2017, p. 174) ; "Infrastructures are conventionally understood as material, technological systems that allow for the interconnection and movement of people, things, and ideas" (Namba, 2017, p. 78). 
sont ainsi vues comme des réalités imposantes - matériellement et spatialement, mais aussi en termes organisationnels ainsi que du point de vue de leur impact économique, social et politique -, marquées par un degré élevé de stabilité et de pérennité, mobilisant pour et affectant par leur fonctionnement (et dysfonctionnement) des masses importantes d'individus qui sont impliqués à divers titres dans la production, distribution et consommation/usage des biens, services et autres activités dont les infrastructures servent justement de supports et de véhicules. Cet accent mis sur les caractéristiques matérielles et organisationnelles de l'infrastructure et son côté imposant, par ses formes et/ou ses effets, ont ainsi fait exclure du champ de l'ouvrage une série de dispositifs matériels mais confinés dans des espaces plus ou moins locaux, ou des réalités qui soutiennent l'action d'une multitude de personnes à travers l'espace mais qui manquent de matérialité forte (au sens ordinaire, physique, du terme), comme les règles de droit, les standards, les schémas classificatoires ou les diverses conventions sociales, par exemple. Cela étant, comme Antoine Picon le rappelle utilement dans sa contribution, la nature fortement matérielle et organisationnelle de l'infrastructure, qui la fait ressembler à un large système "socio-technique " (Thomas P. Hughes), ne doit pas conduire à négliger pour autant les aspects symboliques (« imaginaires ») dont les infrastructures sont souvent vêtues, et qui peuvent, quand un certain nombre de conditions historiques sont réunies, primer même leur côté fonctionnel (un réseau de chemin de fer peu efficace du point de vue économique, pour cause d'absence de trafic suffisant par exemple, peut néanmoins fonctionner comme symbole de modernité pour le pays qui l'a construit) ${ }^{12}$.

\footnotetext{
12 Pour des exemples historiques, mentionnons ici le cas d'une autoroute en Albanie, alors même que l'accès à la propriété privée de voitures était interdit aux Albanais de l'époque (ce cas est étudié par Dimitris Dalakoglou), ou la première "Americanstyle " autoroute au Pakistan, qui n’a pas réussi, à cause même de sa modernité, à attirer beaucoup d'automobilistes (cas étudié par Naveeda Khan) (je cite d'après Namba, 2017, p. 79. L'auteur parle d' "infrastructural fetishes").
} 


\section{Les métamorphoses des infrastructures, entre l'ancien et le nouveau}

Variété des disciplines mobilisées ${ }^{13}$ et des angles d'analyse adoptés ${ }^{14}$, multiplicité des infrastructures abordées et des sites géographiques visités, d'un côté ; une conception générale commune de ce qu'est une infrastructure et des sensibilités, voire des thèses partagées (voir aussi infra), de l'autre : c'est en misant sur ce mixte d'hétérogénéité, permettant de voir large, et d'une certaine unité de vues, atténuant l'intensité des forces centrifuges qui pourraient en résulter, que l'ouvrage propose une première vue panoramique des métamorphoses actuelles du paysage infrastructurel.

Le mot de métamorphose ${ }^{15}$ est choisi à dessein ; il suggère l'idée de changement sur fond de continuités, grâce auxquelles l'objet métamorphosé, dont on souhaite suivre les altérations, préserve son identité (en effet, à défaut d'une telle continuité, ne serait-ce minimale, le nouveau ne porterait en lui aucune trace de l'ancien, et on serait alors en face d'un objet inédit). La métamorphose semble être la modalité de transformation sinon propre à, du moins dominante des infrastructures. Il y a de bonnes raisons pour cela. Réalités massives et imposantes, mobilisant et affectant à travers leur conception, leur fonctionnement, les usages - officiellement consacrés mais aussi clandestins - dont elles sont le théâtre, leur maintenance enfin ${ }^{16}$, une multitude d'acteurs, collectifs et individuels, les infrastructures finissent par avoir une grande emprise sur l'espace qu'elles occupent et la société qu'elles irriguent. Du coup, leur disparition, voire leur transformation radicale, devient une affaire compliquée et un événement peu probable (mais non impossible : il arrive, en effet, à certaines infrastructures de

13 Celles-ci vont de la sociologie à l'aménagement du territoire et de l'architecture à la géographie et à l'histoire.

14 L'infrastructure comme objet propre et comme outil d'enquête aux vertus heuristiques.

15 Notons ici que Nathalie Roseau, qui signe l'un des chapitres de cet ouvrage, avait déjà employé le terme de métamorphose en association avec celui de l'infrastructure dans un texte intitulé de façon significative : «Les métamorphoses de l'infrastructure : New York et l'imaginaire de la ville aérienne» (Roseau, 2007).

16 Sur la question de maintenance des infrastructures, sujet relativement négligé dans la littérature, voir par exemple Graham et Thrift (2007). 
quitter la scène historique, quitte à réapparaître lors d'une nouvelle conjoncture historique) ${ }^{17}$. L'expression de " monopole naturel ${ }^{18}$, que les économistes associent à plusieurs types d'infrastructures, celles en réseaux en particulier, capte sur un mode métaphorique cette emprise dont disposent les infrastructures, capables d'empêcher des concurrents potentiels de pénétrer leur territoire. Mais si les infrastructures meurent difficilement et si leur transformation prend rarement la forme d'un changement radical se produisant d'un seul coup, elles peuvent se métamorphoser sous l'action de forces, selon des formes et à travers des voies que le présent ouvrage tente d'identifier et d'analyser.

L'exemple déjà mentionné de l'autoroute dotée désormais d'une multitude de capteurs et de panneaux à messages variables illustre comment une infrastructure vieille de plusieurs décennies peut être métamorphosée suite à son informatisation, et voir ainsi des rapports, bien établis et de longue date, entre l'entité gestionnaire et les usagers bouleversés. Dans d'autres cas, l'infrastructure peut garder son substrat technique plus ou moins intact et voir le statut juridique de l'entreprise gestionnaire changer. Le rôle, économique et politique, de cette dernière dans le territoire desservi par l'infrastructure peut aussi connaître des modifications substantielles si l'infrastructure dont elle assure le fonctionnement se met au service d'objectifs plus larges que sa fonction originelle. Ailleurs, la métamorphose du paysage infrastructurel peut prendre la forme d'hybridation, avec différents systèmes techniques se développant simultanément et coexistant au sein du même espace. Pour d'autres infrastructures, leur métamorphose peut concerner davantage la structure des organisations associées et la nature des métiers impliqués dans leur conception et leur fonctionnement. Et, comme nous allons le voir, dans le cas de plusieurs infrastructures d'extraction récente - celles

17 Sur ces questions, voir les réflexions, développées par le sociologue Howard S. Becker à partir d'un tout autre terrain, à savoir les « mondes de l'art " (Becker, 2010, ch. 10).

18 Rappelons qu'on se trouve en situation de monopole naturel quand l'efficacité économique est mieux assurée si la production d'un bien ou d'un service est assurée par une seule organisation, et cela grâce à l'existence de rendements croissants. C'est le cas notamment lorsque la production nécessite de lourdes infrastructures (en effet, il serait économiquement absurde d'équiper une ville de plusieurs réseaux d'eau et d'assainissement concurrents). Pendant longtemps, les monopoles naturels étaient des services publics. 
autour de plateformes numériques, par exemple -, le nouveau résulte en grande partie de la reconfiguration de l'existant, la mobilisation originale d'objets, voire d'infrastructures déjà en place.

Le lecteur ne va pas tarder à découvrir par lui-même plusieurs de ces métamorphoses. Permettons-nous une dernière fois de grouper un certain nombre d'entre elles sous deux thèmes particuliers, qu'on va retrouver déclinés dans différentes contributions du recueil.

\section{Une nouvelle place pour l'individu}

Ainsi, pour commencer, plusieurs chapitres sont animés par la conviction que de nouveaux rapports entre l'infrastructure et l'individu sont en train de se nouer devant nos yeux, rapports qui sont traduits par une implication croissante de l'individu dans la conception et le fonctionnement des infrastructures, qu'elles soient déjà bien installées ou prises dans un processus d' "infrastructuralisation " - à savoir, le processus de transformation de dispositifs locaux en infrastructures, via la stabilisation/pérennisation de leur fonctionnement et leur extension/ généralisation par la reproduction de la même unité de base à des échelles spatiales et sociales toujours plus grandes et/ou par la combinaison/ interconnexion de plusieurs systèmes différents ${ }^{19}$.

Qu'entend-on par ces phrases quelque peu sibyllines ? Visitons avec un peu plus de détail un exemple que nous avons déjà introduit, l'automobiliste se déplaçant à travers un réseau équipé de panneaux à messages variables qui l'informent de l'état du trafic. Le même automobiliste avant de se mettre au volant de sa voiture tout comme au long de son trajet ne manquera pas très probablement de consulter parallèlement son GPS ainsi que son smartphone. Ce dernier, connecté à d'autres dispositifs similaires, peut faire bénéficier à son propriétaire des informations détenues par d'autres utilisateurs du réseau, qui sont en train d'échanger en temps réel des données sur l'état de ce dernier ${ }^{20}$.

19 Comme nous l'avons vu, le terme d'infrastructuralisation circule déjà dans la littérature existante (Chu, 2014 ; Day, 2014). Son acception dans ce chapitre doit beaucoup à la contribution d'Alexandre Mathieu-Fritz publiée ici même.

20 Via des applications comme "Connected Citizens » de Waze, par exemple. Il s'agit ici d'un exemple de gestion " communaliste » d'une infrastructure par l'intermédiaire d'un système d'information, qui, contrairement à celui des panneaux aux messages variables, est décentralisé (pour une vue d'ensemble, voir Rifkin, 2016). 
Puisant dans ces sources d'information, notre conducteur sera en mesure de former ainsi sa " propre » infrastructure - réalité dynamique qu'il fait évoluer en fonction des flux de données qui lui parviennent en temps réel -, qui va l'amener entre son point de départ et la destination finale de son voyage. En fabriquant son réseau à lui, qui peut être différent de celui d'un autre conducteur avec qui il partage toutefois point de départ et point d'arrivée, notre individu participe, à côté des acteurs plus imposants, les producteurs des données qui informent ses choix par exemple, au fonctionnement de l'infrastructure.

Cette implication croissante des individus, à la fois moins homogènes et moins cadrés par les normes techniques que par le passé, dans les différents moments de la vie d'une infrastructure, cette fabrication de leur part d'un environnement infrastructurel propre et "sur mesure ", correspondant au mieux à leurs besoins (et aux contraintes qui pèsent sur eux), ne devraient pas être interprétées selon la perspective d'un « jeu à somme nulle " dont chaque acteur n'augmenterait ses prérogatives qu'aux dépens des autres, l'individu enlevant simplement ici à son profit des tâches et des fonctions qui revenaient auparavant à des acteurs spécialisés gravitant autour de l'infrastructure (l'ingénieurconcepteur et la firme-gestionnaire, par exemple) - cela étant, force est de constater que la tentation de faire assurer par les usagers des tâches et des activités qui étaient auparavant de leur ressort n'est pas absente de stratégies poursuivies par quelques gros acteur ${ }^{21}$. Comme l'exemple de l'automobiliste le montre, la nouvelle dialectique entre l'infrastructure et l'individu exige toute une panoplie d'outils et de dispositifs mis

Évidemment, la question de l'efficacité d'un système d'information décentralisé pour la gestion d'une infrastructure en réseau (qui multiplie les interdépendances) reste entière.

21 En avançant cette thèse, nous n'excluons pas la possibilité de situations inverses, où des individus subissent plutôt la montée en puissance de nouvelles infrastructures avec lesquelles ils doivent composer. C'est le cas d'une série d'outils informatiques aux acronymes désormais familiers aux personnels des entreprises et des administrations : ERP (Enterprise Resource Planning, logiciels de gestion intégrés), CRM (Customer Relationship Management), etc. Dans le vaste ensemble des outils présents en entreprise, ces personnels pouvaient faire usage de logiciels, de tableaux Excel ${ }^{\circ}$, voire de fichiers papier tout à fait spécifiques et locaux, ressentis comme bien adaptés aux besoins propres à chacun des métiers. Mais tout cela s'est vu substituer par des outils conçus autour de formats de données uniques sur une large transversalité (voir la contribution signée par Gilles Jeannot et Pascal Ughetto, dans ce recueil). 
à la disposition de ce dernier. Or la production et la distribution de ces outils et dispositifs auprès des individus déclenchent souvent le déploiement d'une série d'actions (nouvelles) de grande ampleur de la part de "gros " acteurs qui interviennent au long de l'arc allant de la conception des infrastructures à leur gestion au quotidien ${ }^{22}$, qu'il s' agisse de changements organisationnels ou de la création de métiers inédits chargés de rendre opératoires les nouveaux rapports entre individu et infrastructure, sans oublier le travail juridique que la puissance publique doit souvent effectuer dans le but d'encadrer justement ces nouveaux rapports.

\section{Le retour du politique}

Un autre thème qui traverse plusieurs chapitres du présent recueil, et qui parfois croise même cet autre fil commun représenté par la nouvelle dialectique entre l'individu et l'infrastructure, est celui du " politique ». Associer politique et infrastructures n'a rien d'inédit. Dans les villes du «Sud » où les infrastructures, rares et souvent au fonctionnement problématique, font l'objet de revendications populaires, voire d'affrontements violents avec les pouvoirs établis, leur caractère éminemment politique n'est que trop visible. Côté " Nord " cette fois, les historiens ont maintes fois exposé les luttes politiques qui ont entouré les réseaux et autres infrastructures pendant longtemps ${ }^{23}$, avant qu'ils ne deviennent par leur diffusion dans le corps social des objets banals, laissés aux mains des techniciens - rappelons que des mouvements politiques

22 Ce qu'on observe à propos des rapports (nouveaux) entre infrastructures et individus peut être lu à la fois comme une illustration locale et comme un site d'observation particulier d'un phénomène bien plus large. Plusieurs sociologues et autres penseurs de la modernité ont, en effet, analysé la montée en puissance de l'individu moderne - cet être autonome, propriétaire de soi, indépendant, capable d'initiatives, se donnant sa propre loi, libéré des chaînes qui liaient de force son homologue prémoderne à des communautés restreintes, dont la famille et la corporation, et au passé via le poids de la tradition... - comme un phénomène à double face : l'individualisation accrue des personnes est inséparable du développement de structures collectives, dont le marché et l'État moderne, qui fournissent justement à la personne les ressources nécessaires et les supports indispensables (salaire, éducation, biens collectifs...) grâce auxquels elle peut entreprendre sa transformation en individu moderne (voir par exemple Castel et Haroche, 2001).

23 Pour le cas emblématique des infrastructures d'eau, dans le "Nord " et dans le "Sud », voir, par exemple, Barraqué (2012). 
particuliers, connus sous les appellations de «municipalisme social» ou de " socialisme municipal », ont fait des réseaux et autres infrastructures urbains une des questions clés de la gestion des villes occidentales à la fin du XIX et aux premières décennies du XX ${ }^{\mathrm{e}}$ siècle (Dogliani, 2002). De même, le rôle, décisif mais souvent peu visible, aux yeux du plus grand nombre du moins, joué par les infrastructures dans la création et le fonctionnement de différentes sortes d'entités politiques, locales comme les villes, mais aussi nationales et transnationales (Lorrain, 2011 ; Badenoch et Fickers, 2010 ; Högselius et al., 2015), a fait l'objet de plusieurs travaux récents. Pourquoi revenir alors à une relation qui semble bien établie dans le monde de la recherche?

Notre conviction est que l'aspect politique des infrastructures, effacé pendant longtemps, dans le «Nord » en tout cas, derrièrel'universalisation des services et des biens délivrés par leur intermédiaire ainsi que par leur invisibilité due à leur (plus ou moins) bon fonctionnement, est en train de refaire surface. Plusieurs causes conjuguent leurs effets pour rendre de nouveau visible le caractère politique des infrastructures. Tout d'abord, la présence accrue de l'individu dans la conception et le fonctionnement des infrastructures à laquelle nous venons de nous référer ne manque pas de donner une plus grande coloration politique aux rapports désormais plus intenses et dynamiques que nous cultivons avec notre environnement infrastructurel. Devenues des sites de risques majeurs et associés à des catastrophes, naturelles ou de nature anthropique, survenues ou à craindre, les infrastructures, surtout celles qui sont labélisées " critiques ", sont en train d'obtenir une visibilité accrue en devenant l'objet de débats intenses dans l'espace public. Le mariage opéré entre les " anciennes " infrastructures avec les nouvelles technologies d'information rend aussi leur gestion bien plus politique que par le passé. En effet, la possibilité pour les acteurs de définir des programmes d'action en temps réel pose en termes nouveaux la question de la légitimité de leur action. Ainsi, alors que les performances des infrastructures étaient souvent en grande partie inscrites une fois pour toutes dans leur conception initiale (leur « hardware»), elles dépendent désormais de décisions prises face à des événements particuliers. Du coup, les auteurs de ces programmes sont soumis aujourd'hui bien davantage qu'hier à l'impératif de l'évaluation et de la justification, aux yeux du public et/ou des instances politiques, de leur action. Les 
infrastructures sont devenues enfin des objets chargés d'un surplus de sens politique par l'intermédiaire d'une série d'enjeux transversaux, comme le développement durable, l'aménagement du territoire, la question de recettes fiscales pour une municipalité ou le "droit à la ville ", voire le "Right to Infrastructure " (qui semble être menacé aujourd'hui même dans le Premier Monde, à en croire les critiques des formes actuelles du capitalisme) (Corsín, 2014). À cause de ces enjeux, plusieurs infrastructures ont, en effet, cessé d'être envisagées comme des réalités isolées, considérées dans leur spécialisation fonctionnelle, laissées aux soins d'organisations spécifiques : si elles sont et demeurent des pourvoyeurs et des supports des biens et des services particuliers, elles se transforment en même temps en outils au service d'objectifs globaux investis par toute une gamme d'acteurs de la scène politique et de la société civile, l'usager final et des mouvements sociaux y compris.

\section{Présentation générale de l'ouvrage}

Si ce qui précède indique quelques grandes avenues qui traversent l'ouvrage, celui-ci peut être aussi parcouru à l'aide d'une multitude de voies qui font découvrir au lecteur plusieurs scènes, grandes et petites, du nouveau paysage infrastructurel, plus diversifié, plus prégnant, plus visible dans son fonctionnement, qui s'étale devant nos yeux, notre Nouveau Monde. Voici donc à destination du lecteur une première carte générale du livre, dessinée grâce à une brève présentation de différentes contributions qui le forment.

La première section de l'ouvrage, intitulée Infrastructure et imaginaire, regroupe quatre contributions. "Infrastructure et imaginaires. Une lecture alternative du changement technique ", signée par Antoine Picon, se présente sous forme d'essai nourri par la profonde culture historique de son auteur. S'appuyant sur une série d'exemples historiques, des chemins de fer au XIX ${ }^{\mathrm{e}}$ siècle au Web 2.0 aujourd'hui, l'auteur propose une conceptualisation originale de l'infrastructure à l'aide d'une lecture tripartite de l'objet : une infrastructure renverrait ainsi à la fois à : 1) une réalité matérielle - l'équivalent du hardware d'un ordinateur -, 2) un ensemble de standards et de règles, de protocoles et d'usages spécialisés permettant de remplir sa mission - ceux-ci constituant en quelque sorte le software de l'infrastructure, pour reprendre l'analogie avec l'univers numérique -, 3) une troisième dimension enfin, l'imaginaire, ou plutôt 
les imaginaires des différents acteurs qui ont affaire à l'infrastructure, depuis les équipes chargées de sa conception jusquà ses utilisateurs ordinaires en passant par les opérateurs qui lui permettent de fonctionner. C'est cette troisième dimension de l'infrastructure qui capte particulièrement l'intérêt d'Antoine Picon, qui voit dans l'imaginaire une voie privilégiée, et peu empruntée jusqu'alors, pour étudier de façon originale la question du changement technique. Centrée sur une infrastructure particulière, le nouvel aéroport de Hong Kong ouvert en 1998, la contribution de Nathalie Roseau ("L'agrandissement de la métropole par l'infrastructure. L'aéroport XXL de Hong Kong ") montre que loin d'être pensé comme un simple dispositif fonctionnel et spécialisé dans ses missions, le nouvel aéroport, couplé avec toute une série d'autres infrastructures, qu'elles soient architecturales ou relevant des transports, avec lesquelles il entre en synergie, marque l'image même qu'on se fait du paysage urbain dans lequel il s'insère et qui ne manque pas de le marquer à son tour : " "City as airport" et "Airport as city" constituent, d'après Nathalie Roseau, les deux faces irréductibles de la figure métropolitaine aujourd'hui incarnée par Hong Kong. " Entrant en résonance avec les contributions précédentes, les deux autres textes qui complètent cette première section de l'ouvrage abordent les rapports, consubstantiels à en croire A. Picon et N. Roseau, entre infrastructures et imaginaire à travers la notion de projet. Projet architectural pour Sarra Kasri ( "Les architectures événementielles, des infrastructures de la ville ?»), projet d'ingénieur dans le cas d'Elsa Vivant (" Naissance d'une infrastructure »). Utilisant comme terrain empirique ce que l'auteur appelle les «Projets présidentiels », à savoir des constructions aspirant à devenir des infrastructures marquant symboliquement à la fois le paysage urbain et le temps historique, S. Kasri se donne comme objectif d'analyser, à travers une série de projets français, mais aussi tunisiens, le processus d'émergence et la carrière sociale d'une architecture à la fois " événementielle " et monumentale, voulus par un "Président " soumis aux exigences de la règle démocratique et obligé de ce fait de recourir au système de concours, garant d'objectivité ; la question des imaginaires multiples, ceux des commanditaires, des concepteurs participant au concours, mais aussi des usagers de ces projets présidentiels constitue alors l'un des éléments cardinaux de l'étude. E. Vivant invite dans sa contribution à lire avec elle la façon dont la romancière Maylis de Kerangal met en récit la construction d'un pont à Coca, petite 
ville ordinaire de Californie. Cette infrastructure romanesque et ses péripéties de construction apparaissent ici comme un moyen d'explorer les transformations territoriales induites, l'articulation des différentes échelles de l'action collective déployée, les modes d'organisation du travail par projet, bref le Nouveau Monde des infrastructures à l'ère de la mondialisation. L'imaginaire de la nouvelle infrastructure est mis en scène à travers le personnage du maire notamment, pour qui le pont de Coca n'est pas qu'une banale construction reliant deux rives, mais l'objet destiné à marquer une rupture dans le récit de sa ville, laquelle, bourgade du Far West pendant longtemps, se projette, grâce à la nouvelle infrastructure, désormais comme métropole aux allures mondiales.

Les rapports, variables, mais toujours extrêmement chargés politiquement et idéologiquement, noués entre les compagnies privées et la puissance publique autour des réseaux urbains sont maintes fois passés au crible de l'analyse de l'historien et d'autres praticiens des sciences sociales. Dans la seconde section de l'ouvrage (Infrastructures urbaines : le public et le privé), un tandem, Dominique Lorrain et Morgan Mouton ("Conglomérats familiaux et infrastructures essentielles. Le cas des Philippines »), et un triumvirat composé de Daniel Florentin, Pauline Gabillet et Catalina Duque Gomez ("La firme d'infrastructures locale et le rééchelonnement ») apportent leurs lots d'analyses sur les relations entretenues par l'" Entrepreneur » et le " Prince » (Defeuilley, 2017) à partir de plusieurs terrains qui nous amènent de l'Asie à l'Amérique latine en passant par l'ex-Allemagne de l'Est et la ville de Grenoble. Une position commune traverse les deux chapitres : penser les relations entre l'intérêt général, dont les institutions publiques sont censées être les garantes, et les intérêts particuliers, poursuivis par le secteur privé, en termes d'opposition frontale peut s'avérer parfois contre-productif pour l'intelligence de ces mêmes relations. Le cas philippin montre, en effet, qu'une poignée d'acteurs privés, sous forme de conglomérats familiaux pour l'essentiel, sont en train de mettre en place et de gérer des infrastructures urbaines en se substituant à la puissance publique - vu de Paris, l'engagement du groupe «Ayala » dans les quartiers centraux de Manilla serait comme si le baron Haussmann et la Compagnie Générale des Eaux avaient fusionné leurs intérêts et modelaient ensemble le centre de la capitale française, écrivent les auteurs -, en affichant même la volonté d'apparaître comme la seule force capable d'emmener le pays 
sur la voie du développement. Comment expliquer cette omniprésence de grands groupes privés dans des activités qui en Occident relèvent de la maîtrise de la puissance publique ? Des trajectoires historiques différentes expliqueraient alors les divergences constatées, répondent nos auteurs, au terme d'une analyse qui fait des infrastructures un outil particulièrement efficace d'analyse comparative. Avec la contribution de Daniel Florentin et de ses coauteurs, le lecteur découvre d'autres configurations possibles du couple " public/privé " en matière d'infrastructures urbaines. Les trois auteurs réunissant et confrontant les résultats de leurs recherches sur les politiques suivies depuis quelques années par trois services municipaux d'infrastructures : le Stadtwerk de la ville de Magdebourg (SWM), organisation assez typique du modèle allemand de gestion des services urbains, dans la mesure où il gère plusieurs réseaux (réseaux d'eau et d'assainissement, mais aussi réseaux d'énergie), le «Gaz Électricité de Grenoble » (GEG) et les «Empresas Publicas » de Medellin (EPM), ces dernières gérant, à l'instar de SWM, plusieurs réseaux urbains pour le compte de la seconde ville de Colombie. Malgré toute une série de spécificités locales, les auteurs décèlent plusieurs évolutions communes. Obligées d'œuvrer désormais au sein de marchés d'énergie très agressifs, libéralisation oblige, les trois organisations ont adopté une politique caractérisée par trois composantes majeures : une extension de leur périmètre d'action, qui les fait sortir de leur base territoriale historique ; une diversification de leurs activités, bien au-delà de leurs missions traditionnelles; et une redéfinition des rapports noués avec leurs villes et municipalités respectives, faisant d'elles des acteurs centraux en matière de politiques urbaines. Suite à cette politique, les trois organisations sont aujourd'hui en mesure de participer bien plus que par le passé au bien-être des habitants de leurs villes, en continuant ainsi d'assurer leur mission de service public, grâce, en grande partie du moins, à la poursuite d'activités lucratives, se comportant alors comme des compagnies privées, déployées en dehors de leur base territoriale historique.

La troisième section ( $L a$ ville post-réseau) vient dans la continuité directe de ce qui précède, tout en déplaçant la focale. Les trois contributions qui la composent se réfèrent, elles aussi, à des infrastructures urbaines sous forme de réseau, mais elles se posent la question de la place que celles-ci sont appelées désormais à occuper dans 
le paysage urbain qui se déploie sous nos yeux. Comme Olivier Coutard et Jonathan Rutherford le rappellent dans leur texte ( Au-delà de la ville des réseaux »), depuis le XIX siècle les réseaux urbains tels qu'on les a connus jusqu'aujourd'hui - à savoir de grands systèmes sociotechniques marqués par une forte centralisation (technique, organisationnelle et financière), fonctionnellement spécialisés, offrant progressivement, sous forme de monopole régulé par la puissance publique, à l'ensemble des citadins des biens et des services standardisés - et le paysage urbain moderne avaient scellé une alliance forte au point de qualifier la ville issue des révolutions industrielles successives de «networked city» (supra). Or, pour un ensemble de raisons, qui peuvent varier d'un contexte urbain à l'autre - faibles croissances économique, démographique et urbaine, contestation des formes d'organisation centralisées et hiérarchisées, préoccupations environnementales qui s'accompagnent d'une injonction à plus de sobriété dans la consommation des ressources -, cette alliance ne va plus de soi ; il en résulte que la forme de grand réseau centralisé a cessé d'être considérée automatiquement comme " the one best way ", économiquement, socialement, écologiquement, pour délivrer des services jugés essentiels aux citadins. Un nombre croissant de configurations sociotechniques, dont plusieurs caractéristiques les placent aux antipodes des grands réseaux centralisés existants - des dispositifs locaux et décentralisés, par exemple -, entrent ainsi en compétition directe ou essaient de vivre en symbiose avec ces derniers, à la fois dans les villes du "Nord » et dans celles du "Sud », où la forme " réseau " n'a pas encore obtenu la place hégémonique qu’elle bénéficie depuis longtemps dans l'Occident riche. Sylvy Jaglin ("Politiques d'infrastructures en Afrique subsaharienne. Le réseau est-il soluble dans la transition urbaine ? ») partage avec les auteurs d' "Au-delà de la ville des réseaux " plusieurs constats et analyses. Mais son terrain d'enquête, les villes africaines, associant depuis longtemps réseau conventionnel et dispositifs hors réseau dans des configurations de fourniture composite, l'incite à formuler une série de thèses marquées par une plus grande circonspection quant à l'émergence d'une ère urbaine définitivement et résolument " post-réseau ". L'auteure considère les configurations de fourniture comme des assemblages urbains constitués de nombreuses entités, humaines et non humaines, qui incorporent les caractéristiques des contextes urbains spécifiquement africains, qu'il s'agisse de leur croissance démographique soutenue parmi les plus fortes du monde, 
de l'émergence de nouvelles demandes et pratiques de consommation en provenance des "classes moyennes ", ou de leur pluralité de normes et de règles. Peu propices à l'homogénéisation des cadres et conditions de l'action collective, ces caractéristiques semblent alors favoriser des assemblages durablement hétérogènes plutôt que des logiques binaires de substitution ou de transition d'une ville pré-réseau vers une ville d'après-réseau. Aïda Nciri ("Infrastructures urbaines et transition bascarbone : spatialiser les processus de diffusion d'innovations de niche. Le cas des réseaux de chaleur en Alberta ») offre au lecteur une étude de cas qui illustre la cohabitation possible au sein du même espace urbain de plusieurs dispositifs sociotechniques, dont le grand réseau traditionnel, fournissant des services urbains. L'auteure a étudié la trajectoire de deux projets récents d'innovation qui visent à équiper les villes canadiennes de Calgary et d'Okotoks avec des systèmes énergétiques alternatifs (il s'agit d'un réseau local de chaleur). Alors que celui d'Okotoks est resté au stade de la démonstration technologique, le projet du downtown de Calgary a réussi à s'étendre en attirant de nouveaux clients. A. Nciri propose alors une série de raisons qui rendraient compte de ces trajectoires divergentes et, ce faisant, elle met, elle aussi, en évidence les rôles multiples que les infrastructures urbaines sont appelées à jouer désormais dans les espaces qu'elles irriguent : loin d'être de simples supports pour la fourniture de services et biens spécialisés, elles deviennent des outils politiques au service d'objectifs globaux, comme la densification et le redéveloppement urbains dans le cas de la ville de Calgary.

La quatrième section de l'ouvrage (Les infrastructures de gestion) nous fait quitter le monde des infrastructures marquant de leur empreinte matérielle le paysage urbain pour l'univers de la (grande) entreprise et de ses infrastructures de gestion. Comme leurs homologues dans les sections précédentes, tous les auteurs qui contribuent à cette partie de l'ouvrage se réferent eux aussi à des réalités imposantes en termes organisationnels et d'impact, mobilisant pour et affectant par leur fonctionnement (et dysfonctionnement) des masses importantes d'individus. Les usages qu'ils font de la notion d'infrastructure n'en opèrent pas moins un certain nombre de déplacements. En s'appuyant notamment sur l'exemple des progiciels intégrés de gestion (ou Entreprise Ressource Planning, ERP) et du Lean manufacturing, Gilles Jeannot et Pascal Ughetto (« Les assemblages d'outils de gestion comme 
infrastructures de connaissance ") ont voulu se frotter à la littérature sur les infrastructures pour explorer le monde des outils de gestion; ceux-ci étant rarement utilisés de façon isolée au sein de l'entreprise, les auteurs parlent d' "assemblages " pour souligner leur mise en relation et de ce qui en résulte. Comment ces " assemblages " émergent-ils ? Qu'est-ce qui les fait tenir ? Quels effets autres que ceux de leurs composantes ont-ils ? Sont ainsi convoquées les réflexions d'un Thomas Hughes sur le développement des Large Technological Systems, celles de Geoffrey Bowker et Susan Leigh Star sur le rôle des standards et, surtout, les développements récents de Paul Edwards sur les « infrastructures de connaissance " ("Knowledge infrastructures»). Pour les deux auteurs, la lecture des outils de gestion avec les lunettes des infrastructures permet de "saisir tout ce qu'il y a de dynamique instable, de modularité dans ces assemblages ", tout en attirant " l'attention sur les échafaudages et le travail d'individus singuliers qui ont été nécessaires à la production d'ensembles qui semblent aujourd'hui se tenir par eux-mêmes ". Malgré tout ce qui sépare les infrastructures urbaines de leurs objets d'étude, G. Jeannot et P. Ughetto croient déceler aussi dans l'univers de la gestion une certaine montée en puissance de l'individu, montée que d'autres auteurs, appartenant à ce recueil ou non, avaient soulignée de leur part à partir d'analyses centrées sur des infrastructures assurant la prestation des services publics. La question de l'individu et ses rapports avec l'infrastructure occupe une place de choix dans la contribution d'Émile Gayoso, Thomas Jammet et Nicolas Klein ("Les plateformes d'innovation, de marque, de conseil. Vers une infrastructure numérique de la relation-client ?»). Les auteurs ont examiné plusieurs dispositifs en ligne mis en place par des grandes entreprises des télécommunications, du transport de voyageurs, de la banque et de l'assurance dans le domaine de la gestion de la relation - client (GRC) - ou plus communément CRM (Customer Relationship Management). Ces enquêtes les ont amenés à se poser la question de savoir si l'on a affaire à un archipel de plateformes numériques relativement indépendantes dans leur rattachement fonctionnel à l'entreprise comme dans leur fonctionnement technique et organisationnel ou si, au contraire, on assiste au spectacle d'une dynamique de généralisation et de standardisation de dispositifs et de pratiques en ligne, autrement dit à la naissance et à la consolidation progressive d'une (nouvelle) infrastructure numérique du lien entrepriseindividu. C'est la thèse d'une extension et d'une transversalité des 
dispositifs en ligne, développés de façon transverse à plusieurs grandes fonctions de l'entreprise (innovation, marketing et vente) et à l'origine des nouveaux métiers de community managers et de webconseillers, que les auteurs défendent. La question des métiers relatifs aux infrastructures numériques est au centre de la dernière contribution de cette section, signée par Denis Duplan et Pascal Ughetto ("Les infrastructures numériques comme problème professionnel»). Les auteurs constatent que si le nombre des utilisateurs des outils numériques ne cesse d'augmenter, l'activité sous-jacente au développement et à l'entretien des infrastructures numériques demeure peu décrite et interrogée. Or ces infrastructures « n' existent en partie que dans la mesure où du travail leur est consacré, un travail qui n'est pas simplement un travail de réalisation et de maintenance des équipements [...], mais aussi d'élaboration des doctrines de ce que doivent être ces infrastructures et les professions légitimes pour les prendre en charge ". Ce sont les directions des systèmes d'information (DSI), habitées par des développeurs de logiciels et des gestionnaires de l'infrastructure matérielle - les devs et les ops dans le vocabulaire indigène -, collaborateurs, mais aussi rivaux, qui constituent le principal terrain d'investigation des deux auteurs, qui soulignent également que tout ne se joue pas au seul niveau des entreprises, les enjeux professionnels autour des infrastructures numériques étant en partie arbitrés par l'État et les politiques publiques.

Tout en restant largement dans l'univers du numérique, les auteurs de l'avant-dernière section de l'ouvrage (Plateformes et infrastructures virtuelles) se penchent sur un certain nombre de dimensions des infrastructures d'aujourd'hui, déjà évoquées pour partie, mais non pas explorées de façon systématique dans les contributions précédentes. À partir de terrains très différents les uns des autres, les signataires des trois textes qui composent cette section ne s'intéressent pas moins à un type particulier d'infrastructures 1) dont le fonctionnement, à l'instar d'Internet, dépend de façon critique d'autres infrastructures déjà en place ; et 2) qui sont les supports de biens et de services livrés de façon essentiellement discontinue dans la mesure où elles sont activées en fonction d'événements particuliers (d'où le caractère virtuel de cette classe d'infrastructures : elles rendent possibles des «transactions " le plus souvent, mais pas toujours, sans qu'une autorité centrale et " experte » intervienne autrement que pour faciliter ces transactions). Patrice Flichy 
(«Les plateformes, étude d'une infrastructure numérique ») s’est penché sur une dizaine de plateformes, allant de la mise en ligne de vidéos au transport des personnes et à l'hébergement de tourisme, en passant par la vente d'objets d'artisan et la réparation à domicile. Alors que ces plateformes sont souvent analysées en termes d'économie collaborative, elles sont traitées par P. Flichy comme des infrastructures, l'accent étant mis sur le mode d'articulation entre l'offre et la demande opérée par ces plateformes, l'organisation des bases de données, les algorithmes à l'œuvre enfin, ces derniers étant qualifiés d'élément infrastructurel clé de la plateforme dans la mesure où ce sont eux qui organisent les transactions entre les internautes. Sensible à la diversité des plateformes en place - P. Flichy distingue ainsi deux types de celles-ci : plateformes de mise en visibilité de l'information et dispositifs de mise en relation d'une offre et d'une demande de biens ou de services -, attentif à leur genèse et à leur évolution, l'auteur met en évidence plusieurs changements dont ces dispositifs ont été à la fois le théâtre et l'acteur. Alors que leur économie s'est d'abord développée dans le secteur non marchand, les plateformes habitent de plus en plus le monde du marché sans perdre pour autant leur rôle d'organisation du travail gratuit ; si des dispositifs locaux continuent à fonctionner, les plateformes se structurent de plus en plus au niveau mondial et défient les régulations étatiques; elles ouvrent enfin de nouvelles frontières à des formes de travail autres que le salariat et le travail indépendant sans pour autant, d'après l'auteur, pouvoir s'ériger en la forme dominante du travail de demain. Valérie November et Servane Gueben-Venière (" La gestion de crise : une infrastructure à part entière ? ") ont décidé de travailler sur une infrastructure bien particulière, invisible la plupart de temps, car activée lors des situations de crises graves, comme un attentat ou une inondation. Avec deux exemples à l'appui - l'attaque terroriste de novembre 2015 à Paris et la crue de la Seine de juin 2016 -, les auteures montrent que l'infrastructure de gestion de crise, si elle s'appuie sur plusieurs autres infrastructures bien identifiées (réseaux de téléphonie, d'électricité, d'hôpitaux, de transports, etc.), ne se contente pas de les utiliser, mais a une identité propre faisant appel à une matérialité et une transversalité dont leur chapitre propose de tracer les contours. Restant "dormant " la plupart de temps, ce type d'infrastructure mobilise, de façon chaque fois variable en fonction de l'événement qui doit être géré, plusieurs éléments constitutifs (humains, matériels, organisationnels, 
décisionnels) qui doivent s'assembler et se déployer efficacement dans l'espace urbain et ceci au moment adéquat. La gestion du temps et, en particulier, la gestion toujours délicate du passage de l'invisibilité à la visibilité figure ainsi parmi ses caractéristiques centrales, et elle la différencie d'autres types d'infrastructure moins sensibles à la dimension du temps réel. Il en va de même pour les questions de la maintenance et de l'amélioration qui se posent aussi en des termes originaux à cause du caractère discontinu du fonctionnement de ce type d'infrastructure : comment, en effet, maintenir les bons réflexes et améliorer l'existant alors que le retour de l'expérience est par définition si rare ? Alexandre Mathieu-Fritz ( Le processus d'infrastructuralisation de la télémédecine et de la télésanté. Vers une endogénéisation des dispositifs dans les standards existants ? ") s'est penché sur deux dispositifs de télémédecine et de télésanté - le premier mettant en lien un CHU et un hôpital gériatrique ; le second, mis en place par un réseau de psychiatres et de psychologues cliniciens, propose via un site Internet des téléconsultations à des patients français expatriés. À l'instar de G. Jeannot et de P. Ughetto dans leur contribution sur les outils de gestion (supra), l'auteur a décidé de recourir à la notion d'infrastructure et à la littérature afférente afin de penser sous un angle nouveau son propre objet d'étude. L'auteur conclut que si les dispositifs de télémédecine et de télésanté actuellement observés peuvent difficilement être considérés, à cause de leur taille réduite et d'une certaine instabilité qui les caractérise pour le moment, comme des infrastructures achevées, ils sont déjà entrés, en revanche, dans un processus d'infrastructuralisation - dans la mesure où les soubassements, industriels, mais aussi organisationnels et juridiques, de ces dispositifs qui concourent à leur stabilisation, pérennisation et extension sont en cours de constitution -, processus qui prend ici la forme d'endogénéisation, notion choisie par l'auteur pour mettre en relief le fait que ces nouveaux dispositifs médicaux viennent se fondre pour une large part dans les standards des infrastructures existantes (comme "Skype " pour la consultation à distance, par exemple).

Deux contributions forment enfin la dernière section de l'ouvrage, qui se veut le pendant de l'introduction générale : cette dernière avait été conçue comme une entrée générale au Nouveau Monde des infrastructures ; la dernière section invite à sortir de ce dernier. Pour ce faire, elle jette des ponts entre les infrastructures d'aujourd'hui 
et une série d'évolutions sociétales plus générales, d'où son titre : "Infrastructures et politique ». Dominique Cardon ("Infrastructures numériques et production d'environnements personnalisés ») revient, pour le développer, sur un thème évoqué dans l'introduction générale : les nouvelles relations que l'individu noue avec l'infrastructure, notamment par l'intermédiaire des techniques de calcul assurées par des dispositifs numériques. À partir de plusieurs terrains - allant des algorithmes mis en œuvre par Google (PageRank) et Facebook (EdgeRank) aux nouvelles pratiques, encore largement expérimentales, dans le domaine de l'assurance automobile (" pay how you drive ») en passant par des politiques de marketing numérique mobilisant des automates de real time bidding-, l'auteur met en évidence une différence, aux multiples ramifications politiques, entre les grandes infrastructures techniques traditionnelles et les infrastructures numériques : alors que celles-là ont souvent été " dédiées à la production d'un espace organisé et régulé pour l'ensemble des bénéficiaires du service ", celles-ci " cherchent à reconfigurer l'espace de façon personnalisée pour chacun d'eux ". Au lieu " de mesurer et d'organiser l'espace pour tous ", les infrastructures numériques, en épousant étroitement les dynamiques d'individuation de nos sociétés modernes, ambitionneraient alors de fabriquer, à partir du comportement et des actions antécédentes de l'individu, "un environnement calculé pour chacun qui se manifeste sous la forme de listes, de recommandations, de guidages géographiques ou de notifications ". En fabriquant pour chaque individu des univers informationnels qui présentent et hiérarchisent l'information différemment en fonction de ses caractéristiques, les grandes plateformes numériques ne manquent pas, d'après $\mathrm{D}$. Cardon, qui mobilise ici les analyses de Michel Foucault sur la "gouvernementalité ", d'exercer sur lui un pouvoir " doux », en intervenant " non pas sur les joueurs du jeu, mais sur les règles du jeu ». On peut se poser, par ailleurs, la question de savoir si la smartification des infrastructures plus traditionnelles, équipées aujourd'hui de toutes sortes de capteurs et gérées à l'aide de dispositifs de calcul numérique, ne produirait pas des effets analogues - pensons à l'impact d'un compteur intelligent ou d'un système d'information en matière de transport sur le comportement de l'usager du réseau en question. Gilles Jeannot ("Les communs et les infrastructures des villes ") s'intéresse à une gamme plus large d'infrastructures, pour partie traditionnelles comme le réseau d'eau, pour partie d'extraction 
plus récente comme ces cartes des services urbains établies de manière collaborative en Afrique à l'aide de smartphones face à l'absence d'offre publique. Il n'en partage pas moins avec D. Gardon le même intérêt pour les questions du pouvoir et du politique. L'engouement récent pour les commons, ces biens matériels partagés dans une communauté et présentés par leurs supporteurs comme un point d'appui et une source de réflexion pour un réinvestissement du politique par la société, offre à G. Jeannot l'occasion de développer un plaidoyer en faveur de la dignité politique des infrastructures urbaines. Contrairement aux " communs ", qui sont par nature l'objet de conflits d'usages qui leur confèrent un degré élevé de visibilité au sein de l'espace public, les infrastructures urbaines, surtout quand elles fonctionnent sans heurts, finissent par disparaître de l'œil du public. Alors que leur universalisation a été le résultat de luttes politiques, souvent acharnées et étalées sur de longues périodes historiques, une fois entrées et installées durablement dans notre quotidien, elles ont vite cessé d'être perçues comme la condensation matérielle des rapports sociaux qu'elles sont pour devenir des objets certes présents par les services et les biens qu'elles délivrent, mais dont le fonctionnement et la maintenance sont laissés aux mains d'organisations lointaines, éloignées du champ de vision de l'usagercitoyen. Or plusieurs événements récents, à l'instar du référendum d'initiative citoyenne de 2011 à Berlin contre des clauses secrètes incluses dans le contrat de concession de l'eau et qui a conduit indirectement à un retrait de l'opérateur privé, témoignent, d'après G. Jeannot, d'une certaine repolitisation de la question des infrastructures. D'où le double appel lancé par l'auteur : aux défenseurs des " communs » à élargir leur espace d'action pour y inclure les services dont les infrastructures urbaines sont les supports privilégiés, aux citadins-citoyens à s'associer, par l'intermédiaire de formes d'action qui restent largement à inventer, à la gestion des infrastructures afin de conférer de nouveau le caractère public à un objet qui n'a jamais cessé de l'avoir dans les faits. 


\section{Références}

Amin A. (2014), "Lively infrastructure ", Theory, Culture \& Society, vol. 31, $\mathrm{n}^{\circ}$ 7-8, p. 137-161.

Badenoch A., Fickers A. (éds) (2010), Materializing Europe. Transnational infrastructures and the project of Europe, Basingstoke, Palgrave Macmillan.

Barraqué B. (éd.) (2012), Urban water conflicts, Paris et Leiden, Unesco et Taylor \& Francis.

Becker H. S. (2010), Les mondes de l'art, Paris, Flammarion.

Bishop R., Phillips J. WP (2014), "The urban problematic II », Theory, Culture \& Society, vol. 31 n $^{\circ}$ 7-8, p. 121-136.

Bowker G. C. (1994), Science on the run. Information management and industrial geophysics at Schlumberger, 1920-1940, Cambridge (Mass), The MIT Press.

Boyer D. (2017), "Revolutionary infrastructure ", dans Harvey P., Jensen C. B., Morita A. (éds) (2017), Infrastructures and social complexity. A companion, New York, Routledge, p. 174-186.

Brenner N. (2004), New state spaces. Urban governance and the rescaling of statehood, Oxford, Oxford University Press.

Carse A. (2012), "Nature as infrastructure: making and managing the Panama Canal watershed », Social Studies of Science, vol. 42, n 4, p. 539563.

Carse A. (2014), Beyond the big ditch. Politics, ecology, and infrastructure at the Panama Canal, Cambridge (Mass.), The MIT Press.

Castel R., Haroche Cl. (2001), Propriété privée, propriété sociale, propriété de soi. Entretiens sur la construction de l'individu moderne, Paris, Fayard.

Chatterjee K., McDonald M. (2004), "Effectiveness of using variable message signs to disseminate dynamic traffic information: evidence from field trails in European cities ", Transport Reviews, vol. 24, n 5, p. 559585.

Chatzis K. (2000), La pluie, le métro et l'ingénieur. Contribution à l'histoire de l'assainissement et des transports urbains (XIX $-X X^{e}$ siècles), Paris, L'Harmattan. 
Chu J. Y. (2014), « When infrastructures attack: the working of disrepair in China ", American Ethnologist, vol. 41, n 2, p. 351-367.

Corsín J. A. (2014), "The right to infrastructure: a prototype for open source urbanism ", Environment and Planning D, vol. 32, n 2, p. 342362.

Coutard O., Rutherford J. (2016), "Beyond the networked city: an introduction ", dans Coutard O., Rutherford J. (éds), Beyond the networked city: infrastructure reconfigurations and urban change in the North and South, New York, Routledge, p. 1-25.

Day R. E. (2014), Indexing it all: The subject in the age of documentation, information, and data, Cambridge (Mass.), The MIT Press.

Defeuilley C. (2017), L'Entrepreneur et le Prince. La création du service public de l'eau, Paris, Presses de Sciences Po.

Dogliani P. (2002), "European Municipalism in the first half of the twentieth century: the socialist network ", Contemporary European History, vol. 11, n 4, p. 573-596.

Donovan K. P. (2015), «Infrastructuring aid: materializing humanitarianism in norhern Kenya ", Environment and Planning D, vol. 33, n 4, p. 732748.

Dupuy G. (1991), L'urbanisme des réseaux : théories et méthodes, Paris, Armand Colin.

Edwards P. N. (2003), "Infrastructure and modernity: force, time, and social organization in the history of sociotechnical systems ", dans Misa T. J., Brey P., Feenberg A. (éds), Modernity and technology, Cambridge (Mass.), The MIT Press, p. 185-225.

Edwards P. N., Bowker G. C., Jackson S. J., Williams R. (2009) "Introduction: An agenda for Infrastructure Studies ", Journal of the Association for Information Systems, vol. 10, n ${ }^{\circ}$ 5, p. 364-374.

Edwards P. N., Jackson S. J., Chalmers M. K., Bowker G. C., Borgman C. L., Ribes D., Burton M., Calvert S. (2012), Knowledge infrastructures: intellectual frameworks and research challenges, Report of a workshop sponsored by the National Science Foundation and the Sloan Foundation, University of Michigan School of Information 25-28 May, http://pne.people.si.umich.edu/PDF/Edwards_etal_2013_Knowledge_ Infrastructures.pdf (consulté le 26 juin 2017). 
Ethnography (2012), numéro spécial : " On infrastructural violence », vol. $13, \mathrm{n}^{\circ} 4$.

Graham S. (2010), «When infrastructures fails », dans Graham S. (éd.), Disrupted cities: when infrastructure fails, New York, Routledge, p. 1-26.

Graham S., Marvin S. (2001), Splintering urbanism:networked infrastructure, technological mobilities and the urban condition, New York, Routledge.

Graham S., Thrift N. (2007), « Out of order. Understanding repair and maintenance ", Theory, Culture \& Society, vol. 24, n 3, p. 1-25.

Graham S., McFarlane C. (éds) (2015), Infrastructuaral lives. Urban infrastructures in context, New York, Routledge.

Guldi J. (2012), Roads to power: Britain invents the infrastructure state, Cambridge (Mass.), Harvard University Press.

Harvey P. (2012), " The topological quality of infrastructural relation: An ethnographic approach ", Theory, Culture \& Society, vol. 29, n 4-5, p. 76-92.

Harvey P., Knox H. (2015), Roads: an anthropology of infrastructure and expertise, Ithaca, Cornell University Press.

Harvey P., Jensen C. B., Morita A. (éds) (2017), Infrastructures and social complexity. A companion, New York, Routledge.

Harvey P., Jensen C. B., Morita A. (2017b), «Introduction. Infrastructural complications ", dans Harvey P., Jensen C. B., Morita A. (éds), Infrastructures and social complexity. A companion, New York, Routledge, p. 1-22.

Högselius P., Kaijser A., van der Vleuten E. (2015), Europe’s infrastructure transition: economy, war, nature, Basingstoke, Palgrave Macmillan.

Howe C., Lockrem J., Appel H. et al. (2016), « Paradoxical infrastructures: ruins, retrofit, and risk ", Science, Technology, \& Human Values, vol. 41, $\mathrm{n}^{\circ} 3$, p. 547-565.

Jensen C. B., "Multinatural infrastructure: Phnom Penh sewage ", dans Harvey P., Jensen C. B., Morita A. (éds) (2017), Infrastructures and social complexity. A companion, New York, Routledge, p. 227-241.

Larkin B. (2013), "The politics and poetics of infrastructure ", The Annual Review of Anthropology, vol. 42, p. 327-343. 
Le Dantec C. A., DiSalvo C. (2013), «Infrastructuring and the formation of publics in participatory design ", Social Studies of Science, vol. 43, n 2, p. 241-264.

Lorrain D. (éd.) (2011), Métropoles XXL en pays émergents, Paris, Presses de Sciences Po.

Lorrain D. (2011b), "La main discrète. La finance globale dans la ville ", Revue française de science politique, vol. 61, nº 6, p. 1097-1122.

Mackenzie A. (2017), «Infrastructures in name only? Identifying effects of depth and scale ", dans Harvey P., Jensen C. B., Morita A. (éds) (2017), Infrastructures and social complexity. A companion, New York, Routledge, p. 379-390.

Mann M. (2012), The sources of social power. Vol. 2. The rise of classes and Nation-states, 1760-1914, New York, Cambridge University Press.

Marié M. (1984), « Pour une anthropologie des grands ouvrages. Le canal de Provence ", Les Annales de la recherche urbaine, n 21, p. 5-35.

Marnot B. (2012), La mondialisation au XIX siècle (1850-1914), Paris, Armand Colin.

Monstadt J. (2009), "Conceptualizing the political ecology of urban infrastructures: insights from technology and urban studies ", Environment and Planning A, vol. 41, p. 1924-1942.

Namba M. (2017), "Becoming a city. Infrastructural fetishism and scattered urbanization in Vientiane, Laos ", dans Harvey P., Jensen C. B., Morita A. (éds), Infrastructures and social complexity. A companion, New York, Routledge, p. 76-86.

Neuman M. (2006), "Infiltrating infrastructures: on the nature of networked infrastructure ", Journal of Urban Technology, vol. 13, $\mathrm{n}^{\circ} 1$, p. 3-31.

Pollock N., Williams R. (2010), « e-Infrastructures: how do we know and understand them? Strategic ethnography and the biography of artefacts ", Computer Supported Cooperative Work, vol. 19, p. 521-556.

Prelorenzo C., Rouillard D. (éds) (2007), Les temps des infrastructures, Paris, L'Harmattan.

Rifkin J. (2016), La nouvelle société du cồt marginal zéro. L'Internet des objets, l'émergence des communaux collaboratifs et l'éclipse du capitalisme, Arles, Babel. 
Roseau N. (2007), «Les métamorphoses de l'infrastructure : New York et l'imaginaire de la ville aérienne ", dans Prelorenzo C., Rouillard D. (éds), Les temps des infrastructures, Paris, L'Harmattan, p. 57-70.

Roseau N. (2016), « Pouvoirs des infrastructures », Histoire urbaine, ${ }^{\circ} 45$, p. 5-16.

Rowland N. J., Passoth J.-H. (2015), "Infrastructure and the state in Science and Technology Studies ", Social Studies of Science, vol. 45, ${ }^{\circ} 1$, p. 137-145.

Royal Academy of Engineering (The) (2012), Smart infrastructure: the future, Londres, The Royal Academy of Engineering.

Star S. L., (1999), "The ethnography of infrastructure ", American Behavioral Scientist, vol. 43, n 3, p. 377-391.

Tarr J. A., Dupuy G. (éds) (1988), Technology and the rise of the networked city in Europe and America, Philadelphie, Temple University Press.

Tousignant N. (2013), "Insects-as-infrastructure: indicating, project Locustox and the sahelization of ecotoxicology ", Science as Culture, vol. 22, n 1, p. 108-131.

Urban Water Journal (2013), numéro spécial : «Real time control of urban drainage systems ", vol. 10, $\mathrm{n}^{\circ} 5$.

Urry J. (2005), "Les systèmes de la mobilité ", Cahiers internationaux de sociologie, $\mathrm{n}^{\circ} 118$, p. 23-35.

Wegrich K., Kostka G., Hammerschmid G. (éds) (2017), The Governance of infrastructure, Oxford, Oxford University Press.

Williamson J. (2008), "A short history of the Washington Consensus ", dans Serra N., Stiglitz J. E. (éds), The Washington consensus reconsidered. Towards a new global governance, New York, Oxford University Press, p. 14-30. 
INFRASTRUCTURE ET IMAGINAIRE 



\title{
Infrastructure et imaginaires
}

\section{Une lecture alternative du changement technique}

\author{
Antoine Picon
}

\section{Les infrastructures : base matérielle, régulation, imaginaires}

On qualifie généralement d'infrastructure un objet ou un système technique de grande ampleur destiné à servir de support à certaines activités, souvent de nature circulatoire comme le trafic automobile ou encore l'échange de données. L'échelle a son importance : pour pouvoir parler d'infrastructure, il faut qu'on se trouve en face d'un objet ou d'un système possédant un caractère imposant soit par sa taille, soit par l'étendue de son déploiement. Un pont apparaît comme une infrastructure ; un réseau téléphonique également. Quant au caractère circulatoire des activités supportées par l'infrastructure, il constitue un cas fréquent, on l'a dit, sans être pour autant systématique, songeons aux murs de soutènement, aux digues ou aux barrages dont la fonction première consiste à retenir la terre ou l'eau.

À partir de cette définition très générale, deux grands types d'approches semblent a priori possibles. La première consiste à privilégier la dimension matérielle de l'infrastructure. L'ingénieur, surtout s'il exerce dans un secteur relativement traditionnel comme le génie civil, sera tenté d'adopter ce point de vue. Terre-pleins destinés à recevoir des containers, routes et ponts lui apparaissent comme des infrastructures. À ses yeux, les standards et les protocoles de nature plus immatérielle qui orientent la conception et organisent l'usage de ces équipements techniques n’appartiennent pas à la même catégorie. Notons immédiatement que cette opposition se révèle déjà beaucoup 
moins pertinente dans un domaine comme le numérique, dans la mesure où les protocoles qui organisent la circulation de l'information comme le TCP/IP de l'Internet jouent un rôle déterminant.

Mais on peut aborder les infrastructures d'une autre manière, sous l'angle des régularités qu'elles introduisent dans les pratiques de ceux qui ont affaire à elles. Dans le cas du pont, par exemple, si l'ingénieur en génie civil étudie avec attention ses dimensions et les techniques employées pour le réaliser, l'usager y voit surtout une commodité de parcours. Aux yeux de ce dernier, les itinéraires qu'il autorise l'emportent sur ses caractéristiques matérielles. Plus généralement, une infrastructure se révèle d'autant plus efficace qu'elle semble en quelque sorte s'effacer derrière les usages qu'elle permet, usages qui se traduisent par toute une série de régularités comportementales. Cet effacement semble atteindre son paroxysme avec les technologies de l'information et de la communication. Cela avait permis au gourou de la Silicon Valley Mark Weiser d'affirmer dans un article demeuré célèbre que " les technologies les plus profondes sont celles qui disparaissent. Elles s'intègrent à la trame de l'existence quotidienne jusqu'à ne plus s'en distinguer »(Weiser, 1991). Cette capacité à s'intégrer à la trame de l'existence quotidienne n'avait pas attendu toutefois le téléphone et l'Internet pour se manifester. Les terre-pleins, les routes et les ponts la possèdent également.

Si les ingénieurs, mais aussi certains historiens des techniques, demeurent attachés à la dimension matérielle des infrastructures, les anthropologues ou les sociologues dont la tâche consiste en priorité à scruter les relations et les pratiques sociales tendent à privilégier les régularités qu'elles induisent. L'alternative tend toutefois à s'émousser sitôt que l'on prête attention à l'existence d'une couche en quelque sorte intermédiaire de standards et de règles, de protocoles et d'usages spécialisés qui permettent à l'infrastructure de remplir ses missions. Entre l'infrastructure comme substrat physique, comme masse de béton et d'acier dans le cas d'un pont, ou encore comme ensemble de circuits électroniques et de câbles dans le cas de l'Internet, et l'infrastructure comme ensemble de pratiques régulières qui voit la dimension technique s'estomper, voire disparaître presque complètement aux yeux de ses 
utilisateurs, s'introduit un ensemble de prescriptions, généralement relayées par des pratiques spécialisées comme celles des opérateurs, sans lequel il serait difficile de parler d'infrastructure.

L'existence de cette couche est évidente dans le cas de l'Internet au point qu'on peut se demander si la véritable infrastructure ne tient pas aux protocoles de type TCP/IP sans lesquels le réseau ne pourrait pas fonctionner. Mais elle s'avère presque aussi déterminante dans le cas des infrastructures traditionnelles. La conception et l'usage des routes et des ponts se révèlent par exemple indissociables des règles qui régissent le trafic automobile ; la chose est encore plus évidente dans le cas des infrastructures de transport en site propre comme le chemin de fer. Les rails ne font pas le réseau ; celui-ci n'existe qu'au travers d'un assemblage de standards, de règles et de pratiques, depuis les vitesses autorisées dont dépend le rayon de courbure maximal des voies jusqu'aux horaires des rames en passant par les pratiques étroitement encadrées des conducteurs, des aiguilleurs ou encore des opérateurs d'entretien. À la suite de Konstantinos Chatzis, utilisons le terme de régulation afin de désigner l'effet de ces standards, de ces règles et de ces pratiques spécialisées (Chatzis, 2000). Il ne saurait y avoir d'infrastructure sans un certain degré de régulation de son usage.

En revenant au numérique, il serait tentant d'utiliser l'analogie entre hardware et software afin de qualifier les relations entre l'infrastructure comme objet ou système technique et l'infrastructure comme ensemble de standards et de règles, de protocoles et d'usages spécialisés qui concourent à sa régulation. En poussant encore plus loin l'analogie et en observant que le software, le système d'exploitation qui tourne sur un ordinateur, qu'il s'agisse de Windows, Mac OS ou encore Linux, s'avère plus déterminant que la configuration matérielle de la machine, on peut aller jusqu'à considérer que c'est la régulation qui fait l'infrastructure. Pourquoi ne pas aller plus loin dans ce cas et étendre la notion d'infrastructure à des dispositifs de régulation faiblement corrélés à une base technique, voire totalement dépourvus de celle-ci : systèmes juridiques ou réglementaires, modèles de gestion, principes d'organisation du travail ? Ce pas en direction d'une conception élargie de l'infrastructure a été franchi par plusieurs contributeurs de cet ouvrage. Tout en reconnaissant l'importance de la régulation, nous préférons quant à nous conserver à l'infrastructure une acception 
plus limitée, renvoyant à une base technologique clairement identifiée à l'exclusion d'infrastructures qui ne seraient pratiquement que du software de gestion et d'organisation. Cette limitation se révèle de toute manière très relative. Car les infrastructures considérées ici peuvent faire appel à une gamme extrêmement étendue de matériaux et de techniques du béton aux fibres optiques, des rails de chemins de fer aux satellites. La part prise par les aspects managériaux et organisationnels peut se révéler d'autre part essentielle. C'est par exemple le cas de la plupart des systèmes de transports.

Base technique et régulation : l'assemblage de ces deux volets permet-il de couvrir l'ensemble des dimensions à l'œuvre dans l'infrastructure au sens défini précédemment? Il nous semble nécessaire de faire intervenir une troisième composante, l'imaginaire, ou plutôt les imaginaires des différents acteurs qui ont affaire à une infrastructure, depuis les équipes chargées de sa conception jusqu'à ses utilisateurs ordinaires en passant par les opérateurs qui lui permettent de fonctionner.

L'imaginaire : pourquoi faire appel à une notion dont la définition souffre toujours d'un certain flou, sans même parler de la difficulté qu'on rencontre lorsqu'on cherche à la manier avec un minimum de rigueur ? Plusieurs raisons justifient à notre sens ce choix, raisons sur lesquelles il nous semble important de s'attarder. On les retrouve à l'arrière-plan de plusieurs études récentes faisant intervenir l'imaginaire dans des disciplines aussi différentes que la philosophie, l'histoire, l'anthropologie, la sociologie ou les sciences politiques ${ }^{1}$.

La première tient à la volonté de faire intervenir « ce que les acteurs ont dans la tête " et cela d'une autre manière qu'en interprétant leurs comportements de manière mécaniste, que ce soit en les réduisant à la maximisation d'une fonction d'utilité, comme le fait la théorie économique, ou en en faisant la simple conséquence d'un jeu social qui leur assignerait en quelque sorte de l'extérieur un rôle à tenir. Des concepteurs aux usagers en passant par les opérateurs, le rapport entretenu avec une infrastructure fait appel à bien d'autres contenus mentaux, à des chaînes d'images en particulier au travers desquelles

1 Par exemple les travaux réunis sous l'égide de la chaire " Modélisation des imaginaires, innovation et création " de l'École Télécom Paris-Tech animée par Pierre Musso. Voir également l'ouvrage coordonné par Sheila Jasanoff et Sang-Hyun Kim (2015). 
s'expriment des désirs et des attentes, ainsi que des valeurs qui contribuent à orienter l'action. Même lorsqu' elle se prétend rationnelle, cette dernière fait intervenir des déterminations irréductibles au calcul. Rien de plus symptomatique à cet égard que les dérives de la pensée stratégique à l'époque de la Guerre froide, dérives dont une équipe dirigée par l'historienne des sciences américaine Lorraine Daston a démontré récemment avec brio les présupposés, présupposés dont certains se révèlent pour le moins déconcertants (Erickson et al., 2013). Paul Edwards avait déjà esquissé la démonstration avec son livre consacré précisément à une infrastructure, le système de défense antiaérienne SAGE, conçu et réalisé par les États-Unis dans les années 1950 au carrefour d'un ensemble de déterminations témoignant là encore de l'influence exercée par des imaginaires à la fois techniques, politiques et anthropologiques, imaginaires véhiculés par toute une série d'acteurs, scientifiques, militaires, ingénieurs (Edwards, 1996).

Un deuxième intérêt des imaginaires tient aux liens étroits qu'ils entretiennent avec la question du projet. La notion de projet connaît un vif succès dans différents mondes professionnels depuis plusieurs décennies. Les entreprises et les administrations ne parlent que de démarche de projet et les design school destinées à favoriser ce type d'approche se multiplient sur le modèle de structures pionnières comme la design school de l'Université Stanford. Paradoxalement, ainsi que le fait observer l'historien des sciences français Frédéric Graber, cette notion n'occupe qu'une place relativement marginale dans les sciences sociales (Graber, 2011). À l'instar de l'anthropologue anglais Tim Ingold, certains auteurs vont même jusqu'à remettre en cause sa pertinence analytique, et cela au nom d'une conception de l'action qui se refuse à séparer intellection et tâches concrètes de transformation de l'environnement humain (Ingold, 2013). S'il entre à coup sûr beaucoup de parti pris dans ce type de position extrême, il n'en demeure pas moins nécessaire d'affiner la définition de la notion de projet afin de pouvoir en faire usage dans les sciences sociales en dépassant notamment une approche encore trop centrée sur la figure de l'individu créateur. Les projets d'infrastructure sont généralement véhiculés par des collectifs et ils portent l'empreinte de leurs imaginaires. Certains imaginaires possèdent de surcroît un caractère autoréalisateur, ainsi qu'on le verra 
un peu plus loin à propos du numérique, c'est-à-dire qu'ils semblent dotés de la capacité de donner naissance à des successions de projets, véritables programmes d'action porteurs de changements concrets.

Le dernier intérêt des imaginaires réside dans la prise différente qu'ils offrent sur la question du politique. D'un côté, les imaginaires contribuent à stabiliser l'ordre existant en reflétant sa structure dans la sphère des représentations imagées. De l'autre, ils facilitent l'éclosion de projets dont certains viennent bousculer cet ordre existant. De ce point de vue, le fonctionnement des imaginaires présente de fortes ressemblances avec celui du couple idéologie/utopie, tel qu'ont pu le caractériser Karl Mannheim ou encore Paul Ricour, si l'on admet avec eux que l'idéologie tend à conforter les institutions en place tandis que l'utopie suggère la possibilité d'une organisation différente de la société (Mannheim, 1936).

Base matérielle, régulation, imaginaires ou encore hardware, software et ontologies, pour utiliser à nouveau une analogie avec l'univers numérique : c'est à l'intersection de ces trois dimensions que nous souhaitons en définitive poser la question des infrastructures. Il nous semble en particulier nécessaire de prendre en compte les imaginaires dans l'analyse de certaines transitions techniques comme l'émergence de la notion de réseau à la charnière des XVIII ${ }^{\mathrm{e}}$ et XIX siècles, ou encore les transformations qu'on cherche à caractériser aujourd'hui en évoquant l'avènement de la smart city ou "ville intelligente".

\section{Contenus imaginaires et notions imaginaires- pratiques : le cas des réseaux}

Comme son nom l'indique, l'imaginaire est peuplé d'images plus ou moins proches de la réalité, auxquelles se trouvent associés des affects, des émotions, des valeurs positives ou négatives. Ces images possèdent un caractère synthétique. À l'instar des rêves, elles peuvent faire cohabiter des éléments contradictoires. Cette capacité se révèle essentielle dans les processus de conception qui font intervenir des phases paradoxales au cours desquelles on attribue des propriétés a priori incompatibles 
entre elles, aux objets ou aux systèmes dont on cherche à définir les caractéristiques. L'innovation passe fréquemment par l'identification et la résolution de tels conflits, on y reviendra par la suite.

Les imaginaires sont partagés par des collectifs de taille variable dont ils contribuent à structurer l'identité. Leur rôle identitaire a été souligné de longue date par les anthropologues et les sociologues (Anderson, 1983). Outre leur caractère plus synthétique qu'analytique et leur capacité à accueillir des éléments contradictoires, les images dont ils sont constitués ont pour propriété d'être liées les unes aux autres par des chaînes le long desquelles s'accumulent les affects, les émotions et les valeurs de manière à produire des effets de sens. Ces chaînes contribuent à structurer les imaginaires à la façon dont un système de géodésiques permet de rendre compte de la géométrie d'une surface. En se croisant, elles esquissent des topologies, avec des zones saillantes et des dépressions, des régularités et des singularités, des effets de rapprochement et d'éloignement. Ces topologies peuvent être interprétées comme des sortes de paysages où se mêlent réalité et fiction et qui permettent à des récits de se constituer. Leur fonction matricielle fait de ces chaînes d'images et de ces topologies ou paysages les contenus véritables de l'imaginaire. De tels contenus sont généralement appréhendés par les observateurs extérieurs au travers des récits qu'ils engendrent. Dans le cas des infrastructures, certains des récits tenus par leurs concepteurs concernent la réalisation de projets qui se veulent des améliorations parfois radicales de l'existant. Notons dès à présent que ce type d'imaginaire n'est pas le seul à se développer à propos des infrastructures. L'imaginaire des concepteurs n'est pas celui des opérateurs ou celui des usagers, même s'ils partagent souvent des images, des chaînes imagées, des topologies et des récits qui acquièrent du même coup un caractère dominant. Un certain nombre d'imaginaires se caractérisent en outre par la rupture qu'ils opèrent par rapport aux contenus imaginaires dominants. Dans le cas des infrastructures toujours, signalons l'existence d'imaginaires du détournement d'usage ou encore de la déconnexion. Les imaginaires de la déconnexion n’ont d'ailleurs jamais été aussi présents dans le débat public qu'à l'heure où le numérique semble triompher sans partage ${ }^{2}$.

Ainsi qu'on pourra facilement s'en convaincre en se promenant sur le site de la librairie en ligne d'Amazon, cet imaginaire s'exprime notamment au travers de la 
Certains contenus imaginaires sontà l'origine de notions qui possèdent une portée pratique toute particulière. Cette portée pratique explique qu'on ait tendance à naturaliser ces notions en oubliant au passage ce qu'ils doivent à l'imaginaire. Nous les appellerons notions imaginairespratiques. Parmi leurs caractéristiques, notons leur inséparabilité de récits et de projets dont les racines imaginaires se trouvent là encore fréquemment occultées. Dans le domaine des infrastructures, le réseau fait à notre sens partie de ces notions imaginaires-pratiques, même si son usage intensif conduit souvent à considérer que cette catégorie d'infrastructures a toujours existé, alors même qu'on connaît assez bien les circonstances qui ont entouré son émergence.

Dans le cas français, mais la plupart de ses enseignements sont susceptibles de généralisation, l'émergence d'une lecture en termes de réseaux des infrastructures de transport et des systèmes urbains, se fait jour en moins d'un siècle, entre 1750 et les années 1820-1830 pour être plus précis, sous l'effet d'un certain nombre de transformations concrètes. Les routes et les canaux, puis des infrastructures urbaines comme l'adduction d'eau et l'assainissement, vont être tout d'abord pensés comme des systèmes intégrés au lieu d'être envisagés comme des juxtapositions de tronçons relativement indépendants les uns des autres (Lepetit, 1992). L'essor de cette pensée systémique s'appuie sur de nouvelles pratiques techniques, comme l'adoption des chaussées à la Mac Adam dans les années 1820, qui conduit à réorganiser l'entretien des routes. Surtout, les structures professionnelles évoluent, avec par exemple en France la création d'un corps spécialisé d'ingénieurs d'État chargé de la construction et de la maintenance des voies de communication territoriales, le corps des Ponts et Chaussées, qui étendra par la suite ses compétences à l'infrastructure technique de grandes villes comme Paris (Picon, 1988). En matière de routes, les cantonniers de station font leur apparition dans les années 1830 tandis que les compagnies de transport évoluent pour s'adapter à l'organisation en réseau de l'infrastructure routière. De nouveaux savoirs se constituent en parallèle : savoirs techniques, administratifs, mais aussi économiques avec le développement des premiers calculs concernant l'« utilité » des infrastructures.

prolifération d'ouvrages se proposant comme des guides de la vie " off the grid ", c'est-à-dire déconnectée des principaux réseaux d'énergie et d'information. 
Mais cette évolution n'aurait pas été possible sans une mutation tout aussi profonde des représentations des infrastructures fondée sur l'association de deux types d'images clefs : celle d'une trame régulière faisant songer à la maille d'un filet de pêche - comme l'anglais network, le mot français "réseau " dérive d'un terme employé pour désigner un filet -, celle surtout du système sanguin dont la circulation vient vivifier le corps (Guillerme, 1988). L'imaginaire fonctionne au moyen de chaînes d'images le long desquelles se propagent et s'enrichissent les effets de sens au point d'acquérir la même évidence que des réalités plus tangibles. Au travers de la figure du système des artères et des veines, le territoire puis la ville apparaissent progressivement comme des sortes d'organismes qui dépériraient sans les diverses circulations dont les infrastructures constituent les supports. La métaphore circulatoire renvoie à son tour à un imaginaire beaucoup plus général du flux et de ses bienfaits dans la nature comme en société. Les Lumières dans leur ensemble portent la marque de cet imaginaire circulatoire. "Tout est sans cesse en mouvement dans la nature ; tous les corps sont dans une continuelle fluctuation ", peut écrire le médecin et philosophe PierreJean-Georges Cabanis, avant d'ajouter qu'une telle circulation conserve "l'éternelle jeunesse de l'univers " (Cabanis, 1980). Les bienfaits de la circulation naturelle sont censés être prolongés et amplifiés par la circulation des hommes et des marchandises. L'ingénierie, comme la pensée économique naissante, s'enracine dans cet imaginaire des réseaux et des flux qui donne naissance à toute une série de récits concernant le caractère vivifiant des circulations.

Dès le départ, la notion de réseau possède une dimension évidente de projet. Comme le rappelle le géographe urbain Gabriel Dupuy, la genèse d'un réseau correspond presque toujours à un projet de transaction (Dupuy, 1991). Quelque chose est censé circuler et s'échanger entre les nœuds du réseau, qu'il s'agisse de véhicules, d'eau, d'énergie ou encore d'information. Un tel projet revêt presque inévitablement une portée sociale et politique. Le « laissez faire, laissez passer » des Lumières imprègne la pensée naissante des réseaux de transport. Ce sont d'ailleurs les mêmes administrateurs " éclairés " qui tentent de promouvoir la construction de routes et de canaux modernes et le laisser-faire économique. La transition qui mène d'un ensemble de voies terrestres 
et navigables pensées comme des tronçons indépendants à une série de mailles couvrant le territoire se révèle ainsi indissociablement technique et politique.

L'infrastructure au sens physique du terme ne fait pas le réseau. À côté des routes et des canaux, les phares offrent un autre exemple, peutêtre plus révélateur encore, de la transition permettant de passer d'une série d'équipements techniques à un réseau. Longtemps conçus comme des tours de signalisation isolées, les phares se voient progressivement réinterprétés comme les nœuds d'un réseau d'éclairage et de balisage des côtes de France au cours de la première moitié du XIX ${ }^{\mathrm{e}}$ siècle. Ainsi que l'a montré l'historien des phares Vincent Guigueno, une telle évolution est là encore tributaire d'innovations techniques comme les lentilles de Fresnel, de pratiques d'entretien, de cultures professionnelles d'administrateurs et d'ingénieurs, de constructeurs, de navigateurs et de gardiens (Guigueno, 2001).

L'exemple des réseaux vient en définitive à l'appui de la lecture tripartite de l'infrastructure que nous avons proposée. À partir d'une base matérielle, chaussées, canalisation, tours de signalisation ou encore câbles et serveurs, se déploie un second niveau d'organisation professionnelle et de pratiques sociotechniques stabilisées, niveau auquel s'opère la régulation des infrastructures. L'imaginaire constitue enfin un troisième niveau distinct des deux précédents. Mais il ne faut pas concevoir ce dernier comme au-dessus des deux autres. Il se situe bien plutôt à leur articulation ; il contribue à leur ajustement tout en contribuant à leur insuffler une portée politique plus générale que celle qui naît des services assurés au jour le jour par l'infrastructure. Pour prendre un exemple concret, le caractère politique d'un réseau de chemins de fer ne réside pas uniquement dans le fait qu'il dessert ou non telle ou telle localité ou dans la sélection qu'il opère de facto parmi les publics auxquels il serait susceptible de s'adresser par l'intermédiaire de ses règles de tarification. Les contenus imaginaires dont s'accompagnent sa conception et son usage, les notions imaginaires-pratiques qui contribuent à sa structuration, à commencer par les déclinaisons particulières du réseau dont il porte l'empreinte, possèdent également une dimension politique, celle d'un ensemble de projets de transactions jamais complètement réalisés et qui ne prennent véritablement fin qu'avec son démantèlement. 
Le chemin de fer se révèle paradigmatique de l'organisation tripartite des infrastructures. Au premier niveau, les rails, les gares et les locomotives. Au second, les règles d'exploitation, les horaires, les habitudes des voyageurs. Au dernier, une série d'images et de valeurs cristallisées pour partie autour de la notion de réseau et des différentes déclinaisons qu'en élaborent les administrateurs, les ingénieurs ou encore les usagers du chemin de fer. Parmi les évidences partagées par ces différents acteurs du réseau, une lecture en termes de circulation et de flux. Depuis le XVIII ${ }^{\mathrm{e}}$ siècle, la plupart des infrastructures se veulent circulatoires et leur régulation consiste pour l'essentiel à organiser les flux dont elles constituent le support. Comme les infrastructures territoriales, les infrastructures urbaines possèdent la même structure tripartite. À l'instar des grands réseaux territoriaux, les réseaux urbains ont été dès le départ pensés en termes de flux, et cela bien avant l'entreprise de théorisation d'un Manuel Castells.

\section{Changement technique et imaginaire}

La place des imaginaires dans les processus de changement technique se trouve systématiquement sous-estimée, et cela pour au moins deux raisons. La première tient à la difficulté d'assigner une place précise à une notion qui demeure, on l'a dit, entachée de flou. Animées d'un désir d'apparaître aussi objectives que les sciences de la nature, les sciences sociales préfèrent d'autre part se placer sur le terrain apparemment plus stable des déterminations socio-économiques. L'exemple des infrastructures peut inciter toutefois à dépasser ce qui ressemble beaucoup à une forme de positivisme qui n'ose pas dire son nom.

Les imaginaires jouent tout d'abord un rôle de tout premier plan dans les processus de conception. Ils permettent d'explorer des combinaisons a priori impossibles, de s'abstraire temporairement de certaines contraintes. Le célèbre " Architectes. [...] Oublient toujours l'escalier des maisons " du Dictionnaire des idées reçues de Flaubert se révèle beaucoup plus profond qu'un simple trait d'humour (Flaubert, 1952). Car il faut souvent oublier pour un temps qu'il faut des escaliers pour imaginer des dispositifs spatiaux innovants. 
Les imaginaires permettent à des acteurs et à des communautés professionnelles de se constituer. Dans la France des Lumières, ils jouent par exemple un rôle déterminant dans la structuration du corps des ingénieurs des Ponts et Chaussées en charge des voies de communication. Les ingénieurs de ce corps se distinguent davantage par un ensemble de représentations et de valeurs partagées concernant par exemple la nécessité de mettre en mouvement les hommes et les marchandises que par des savoirs qui demeurent en fait assez proches de ceux des architectes (Picon, 1988). Les imaginaires occupent une place tout aussi déterminante aujourd'hui dans l'émergence de certains des nouveaux acteurs de la ville intelligente comme ces programmeurs-développeursentrepreneurs que l'urbaniste américain Anthony Townsend qualifie de " civic hackers" dans un livre récent (Townsend, 2013).

Par-delà son impact sur des milieux professionnels restreints, l'imaginaire concerne bien d'autres acteurs, usagers des techniques notamment. Dans les processus de changement technique, il permet à des désirs et à des souhaits de se cristalliser. Au cours des dernières décennies, ce rôle a pris un nouveau relief avec le caractère autoréalisateur des imaginaires numériques, caractère que nous avons cherché à mettre en évidence à propos des logiques qui conduisent à la ville intelligente (Picon, 2015). Nos villes sont en train de devenir intelligentes parce que toute une série d'acteurs désire qu'il en soit ainsi, de la même façon que l'informatique ubiquitaire a vu le jour à la charnière des années 19902000 sous l'effet d'un ensemble de discours autoréalisateurs de la Silicon Valley que sont venues relayer expérimentations et innovations. Un tel caractère autoréalisateur se révèle ordinairement au cours de phases de changement technique accéléré. Avant le numérique, l'avènement du chemin de fer ou encore la diffusion de l'électricité se sont accompagnés du développement d'imaginaires autoréalisateurs, l'électricité surtout qui est contemporaine d'un essor sans précédent de la littérature d'anticipation (Beltran et Carré, 1991).

Mais la principale contribution des imaginaires au changement technique pourrait bien résider dans l'émergence de notions imaginairespratiques comme celle de réseau qui a coïncidé avec une réorganisation profonde des pratiques de régulation associées aux infrastructures territoriales et urbaines. Certes, on pourrait s'interroger sur ce qui vient en premier, la réorientation des imaginaires ou la transformation des 
pratiques. Est-il toutefois si nécessaire que cela de chercher un terme premier dont l'autre dériverait ? Il est peut-être plus intéressant de parler de " coproduction " des imaginaires, des pratiques de régulation et enfin d'un ensemble plus général de régularités sociales situées en aval des pratiques de régulation, à la façon dont Sheila Jasanoff parle de coproduction de la science et du social (Jasanoff, 2004). L'exemple des réseaux incite en revanche à placer certaines innovations techniques en aval de cette coproduction. Il est frappant par exemple que les chemins de fer se soient développés peu après l'émergence de la notion de réseau et la transformation des pratiques de régulation des infrastructures, comme si le passage de la curiosité de foire d'une machine à vapeur montée pour la première fois sur des roues et circulant sur des rails métalliques que propose l'ingénieur anglais Trevithick en 1808 avec sa "Catch-Me-Who-Can » à une infrastructure de transport qui va révolutionner les sociétés de l'ère industrielle naissante avait nécessité l'évolution préalable des imaginaires et des pratiques de régulation.

\section{Des flux aux événements : vers un nouvel imaginaire du web 2.0 et de la ville intelligente ?}

Interrogeons-nous, pour terminer, sur les notions imaginairespratiques qui émergent aujourd'hui en relation avec le développement du Web 2.0 et la montée en puissance de la thématique de la ville intelligente. Nous ferions volontiers l'hypothèse que la notion de " plateforme ", qui a connu un vif succès ces dernières années auprès des spécialistes des infrastructures numériques et que l'on accole généralement à des systèmes techniques comme ceux de Google, Amazon ou encore Uber, fait partie de ces entités imaginaires-pratiques qui viennent donner forme aux désirs d'innovation contemporains ${ }^{3}$. La ville intelligente est souvent présentée comme reposant sur le développement de plateformes.

Certains chercheurs parlent d'ailleurs aujourd'hui plutôt de plateforme que d'infrastructure. On trouvera une discussion approfondie de cette question dans Jean Christophe Plantin, Carl Lagoze, Paul N. Edwards, Christian Sandvig, "Infrastructure Studies Meet Platform Studies in the Age of Google and Facebook ", New Media \& Society, pré-publication, 2016, http://www.academia. 
Celles-ci témoignent d'un glissement progressif de la gestion globale des flux, qui avait caractérisé les infrastructures de l'ère industrielle, à une approche beaucoup plus fine, moléculaire ou atomistique si l'on veut d'événements élémentaires consistant en mise en relation de requêtes et d'offres de biens et de services suivant des protocoles prédéterminés. Ces requêtes peuvent être principalement informationnelles dans le cas de Google, concerner des biens culturels et des équipements en ce qui concerne Amazon, des demandes de transport ou d'hébergement s'agissant d'Uber, Lyft ou Airbnb, l'important étant de reconnaître leur caractère fondamentalement événementiel. Une rencontre entre demande et offre se trouve organisée par l'intermédiaire d'une plateforme numérique. Cette rencontre est enregistrée par la plateforme qui peut ensuite s'en servir comme d'une ressource afin de mieux ajuster la nature de ses offres et l'architecture de ses protocoles.

Plus généralement, le développement du numérique conduit à la multiplication d'enregistrements concernant de micro-occurrences assimilables à autant d'événements ponctuels, qu'il s'agisse de mesures environnementales comme le degré de pollution de l'air, de données de géolocalisation ou de consommations individuelles au sein d'un réseau électrique ou téléphonique. Tout se passe comme si les entités fortement agrégées auxquelles se référaient les infrastructures traditionnelles se trouvaient en quelque sorte pulvérisées pour donner naissance à des milliards d'informations concernant ces occurrences et événements élémentaires. Les big data dont il est si souvent question aujourd'hui ne constituent dans la plupart des cas que des traces de ces événements ${ }^{4}$. Dans ce contexte, on assiste à l'émergence d'un nouvel imaginaire technologique et urbain de " ce qui arrive " à la pensée essentiellement circulatoire qui avait vu le jour à la charnière

edu/27555302/Infrastructure_studies_meet_platform_studies_in_the_age_of_ Google_and_Facebook (consulté le 7 mars 2017).

4 Voir par exemple sur ce thème Dominique Boulier, "Les sciences sociales face aux traces du big data ? Société, opinion et répliques ", 10 avril 2015, https://halshs. archives-ouvertes.fr/halshs-01141120/document (consulté le 7 mars 2017).

5 Paul Virilio avait eu l'intuition de ce caractère de plus en plus événementiel, même s'il le réservait à de macro-événements catastrophiques tels que les attentats terroristes ou les désastres écologiques, dans Ce qui arrive, Paris, Fondation Cartier pour l'art contemporain, 2002. Nous avons développé cette question dans les deux ouvrages que nous avons consacrés aux villes intelligentes. 
des $\mathrm{XVIII}^{\mathrm{e}}$ et $\mathrm{XIX}^{\mathrm{e}}$ siècles, en même temps que se mettait en place la notion imaginaire-pratique de réseau. Il reste à étudier plus finement les implications de cet imaginaire en ce qui concerne les infrastructures et les projets dont elles font l'objet. Réseaux ou plateformes témoignent en définitive de l'interaction étroite entre substrat technique, régulation et imaginaire qui est au fondement même de la catégorie hétérogène de ces objets et de ces systèmes que nous qualifions d'infrastructure.

\section{Références}

Anderson B. (1983), Imagined Communities: Reflections on the Origin and Spread of Nationalism, Londres, Verso.

Beltran A., Carré P. A. (1991), La fée et la servante. La société française face à l'électricité XIX $-X X^{e}$ siècle, Paris, Belin.

Chatzis K. (2000), La pluie, le métro et l'ingénieur : contribution à l'histoire de l'assainissement et des transports urbains, XIX'-XX siècles, Paris, L'Harmattan.

Cabanis P.-J.-G. (1980), Rapports du physique et du moral de l'homme [1802], rééd. Paris, Genève, Slatkine.

Dupuy G. (1991), Lurbanisme des réseaux : théories et méthodes, Paris, Armand Colin.

Edwards P. (1996), The Closed World: Computers and the Politics of Discourse in Cold War America, Cambridge (Mass.), The MIT Press.

Erickson P., Klein J. L., Daston L., Lemov R., Sturm T., Gordin M. D. (2013), How Reason Almost Lost its Mind. The Strange Career of Cold War Rationality, Chicago, The University of Chicago Press.

Flaubert G. (1952), Dictionnaire des idées reçues, dans Flaubert G., Euvres, Paris, Gallimard, La Pléiade, t. 2, p. 991-1023 (p. 1000 en particulier).

Graber F. (2011), « Du faiseur de projet au projet régulier dans les Travaux Publics (XVIII $-\mathrm{XIX}$ siècles) : pour une histoire des projets ", Revue d'histoire moderne et contemporaine, vol. 58, $\mathrm{n}^{\circ} 3, \mathrm{p} .7-33$.

Guillerme A. (1988), "L'émergence du concept de réseau 1820-1830 ", dans Dupuy G. (éd.), Réseaux territoriaux, Caen, Paradigme.

Guigueno V. (2001), Au service des phares. La signalisation maritime en France $X I X^{e}-X X^{e}$ siècle, Rennes, Presses universitaires de Rennes. 
Ingold T. (2013), Making: Anthropology, Archeology, Art and Architecture, Milton Park, Abingdon, Oxon, Routledge.

Jasanoff S. (éd.) (2004), States of Knowledge: The Co-Production of Science and the Social Order, New York, Routledge.

Jasanoff S., Sang-Hyun K. (2015), Dreamscapes of Modernity: Sociotechnical Imaginaries and the Fabrication of Power, Chicago, University of Chicago Press.

Lepetit B. (1992), Les villes dans la France moderne (1740-1840), Paris, Albin Michel, 1988.

Mannheim K. (1936), Ideology and Utopia: An Introduction to the Sociology of Knowledge, Londres, Paul Trench, Trubner \& Co.

Picon A. (1988), Architectes et ingénieurs au Siècle des Lumières, Marseille, Parenthèses.

Picon A. (1992), L'invention de l'ingénieur moderne : l'École des Ponts et Chaussées, 1747-1851, Paris, Presses de l'École nationale des Ponts et Chaussées.

Picon A. (2015), Smart cities. Théorie et critique d'un idéal auto-réalisateur, Paris, Éditions B2.

Picon A. (2015), Smart Cities: A Spatialised Intelligence, Chichester, Wiley.

Ricour P. (1986), Lectures on Ideology and Utopia, New York, Columbia University Press.

Townsend A. (2013), Smart Cities: Big Data, Civic Hackers, and the Quest for a New Utopia, New York, Londres, W.W. Norton \& Company.

Weiser M. (1991), "The Computer for the 21st Century ", Scientific American, vol. 265, n³, p. 94-104 (p. 94 en particulier). 


\title{
L'agrandissement de la métropole par l'infrastructure
}

\section{L’aéroport XXL de Hong Kong}

\author{
Nathalie Roseau
}

Vilipendée comme lieu critique des failles de nos sociétés urbaines, l'infrastructure est aussi brandie comme rempart d'un urbanisme volontaire. Elle occupe un rôle prégnant dans les projets contemporains d'agrandissement métropolitain, comme en témoignent l'actualité du Grand Paris mais aussi celle de nombre de "métropoles XXL " (Lorrain, 2011 ; Roseau, 2012). Comment concrétise-t-elle ce processus d'agrandissement ? Et que dit ce mouvement de sa nature propre ? Pour comprendre ce méta-objet, sa construction et sa mutation, nous nous intéresserons au tournant qu'a connu à partir des années 1990 l'infrastructure aéroportuaire de Hong Kong dans le contexte d'une transformation profonde de la cité-État du sud-est du delta de la Rivière des Perles.

L'exemple de Hong Kong est à la fois atypique et emblématique. La création de son nouvel aéroport relève d'abord de l'exception. En un temps court, l'espace de neuf ans, s'est accompli un ambitieux programme qui a radicalement modernisé la ville-archipel. La réalisation de cette entreprise se mesure à son articulation étroite avec la planification urbaine qui a favorisé d'importants réaménagements au-delà de l'infrastructure stricto sensu. Enfin, l'événement est aussi géopolitique puisqu'il s'inscrit dans le cadre du repositionnement régional de Hong Kong. La décision relative à l'aménagement du nouvel aéroport intervient dix ans après la mise en place fin 1978 par Deng Xiao Ping des Zones économiques spéciales de Shenzhen et de Zhuhai dans le sud du delta de la Rivière des Perles et quelques années avant l'échéance en 1997 de la rétrocession de la colonie britannique à la République populaire de Chine. 
The Shape of things to come : le titre de l'œuvre de Herbert George Wells auquel le gouvernement de Hong Kong recourt en 1989 pour figurer le dessein poursuivi, est éclairant pour caractériser la ville que les autorités projettent : celle qui advient et sur laquelle ils entendent imprimer leur marque ${ }^{1}$. L'incarnation de ce futur volontaire repose sur l'instrument infrastructurel dont nous verrons qu'il se déploie, protéiforme, comme une pieuvre dans l'espace hongkongais. Si l'on peut a priori parler d'infrastructuralisation du milieu, le dispositif projeté, en même temps qu'il modifie la géographie urbaine, se modèle selon son contexte, la ville le colonisant, l'un et l'autre ne formant qu'une seule scène dont les frontières se déplacent et se recomposent. Ce processus se poursuit encore dans le contexte de l'expansion régionale du delta de la Rivière des Perles. De la métropole à la mégalopole : ces « quantum laps $»^{2}$, comme la cité-État les nomme, font de l'infrastructure un levier qui lui permet de se renouveler, transformant à chaque fois son propre système de référence territorial, politique, opérationnel.

Maintes fois soulignée dans la littérature scientifique, la dimension inédite de ce processus de mutation infrastructurel, urbain et régional ne saurait occulter les dynamiques parfois contradictoires qui le travaillent ${ }^{3}$. Si la figure aéroportuaire n'a cessé d'occuper une place dominante dans la littérature des Urban studies consacrée à l'hypermodernité, ce retour sur l'histoire de l'infrastructure aérienne de Hong Kong nous permettra de situer la question de la "ville-aéroport ", à distance des catégories la caractérisant habituellement - le "non-lieu " ou l' "Aérotropolis " par exemple (Augé, 1995 ; Castells, 1996 ; Ibelings, 2002 ; Thackara, 2005 ; Kasarda et Lindsay, 2011). L'étude qui suit vise à cerner d'une part les spatialités de l'infrastructure aux échelles qu'elle irrigue, d'autre part les causalités complexes par lesquelles ces espaces se construisent.

1 Hong Kong's Port and Airport Development Strategy: A Foundation for Growth, Hong Kong, Government Information Services, octobre 1991, p. 67.

2 «sauts quantiques", Ibid., p. 68.

3 La création d'un territoire par le programme aéroportuaire n'est pas une nouveauté, comme l'atteste la construction de l'aéroport d'Osaka au Japon sur une île artificielle au large de la baie du Kansai, dont le chantier s'est achevé en 1994 (Nadal, 1992). À propos de l'exemple hongkongais, cf. Matsuda (1997), Orsay-Lam (2002), McNeill (2014). 
"Setting the scene " : comme le sous-titrent les auteurs de la stratégie qui va donner naissance au projet - la Port and Airport Development Strategy -, les dés sont jetés de manière quasi théâtrale, sur une table dont les fondations sont politiques, faisant du dit l'un des instruments $\mathrm{du}$ processus à venir de transformation de l'infrastructure. Rompre avec l'amnésie spatiale et temporelle dont pâtissent les instruments qui façonnent le futur des villes : c'est en remontant le fil de l'analyse des images et des discours produits par les autorités, les constructeurs, les promoteurs, la presse, les experts et les critiques, que nous souhaitons observer la métamorphose de la ville-archipel en nous intéressant aux discours et aux projets, ainsi qu'à leurs effets sur ce qui advient.

\section{Une longue gestation}

Établi à l'origine dans la baie de Hong Kong, sur la péninsule de Kowloon, l'aéroport originel de la ville, Kai Tak, actif depuis 1924, ferme ses portes le 5 juillet 1998 à minuit, le transfert s'opérant dans la nuit vers le nouvel aéroport, créé sur l'île artificielle de Chek Lap Kok, à 34 kilomètres de l'hypercentre. Pour comprendre les origines de cet événement, il convient d'abord d'en rappeler les prolégomènes.

Envisagé par le gouvernement britannique dès 1946, le déménagement de l'aéroport constitue un sujet récurrent. Pris dans un faisceau de contraintes urbaines, le site de Kai Tak est bridé dans son développement. Aéronefs plus lourds et plus rapides, essor prévisible du trafic commercial, opérations plus complexes : au lendemain de la guerre, les mutations du transport aérien modifient la donne pour les aéroports, tandis que l'urbanisation de l'archipel connaît une croissance fulgurante, stimulée par les événements politiques que vit son grand voisin chinois. L'arrivée au pouvoir de Mao Zedong, et les politiques de répression qui s'ensuivent engendrent des vagues d'immigration dans l'archipel sous statut britannique ${ }^{4}$.

De 1945 à 1950, la population de Hong Kong croît de 750000 habitants à 2200000 habitants. Le rythme de cette croissance se poursuivra jusqu'aux années 1980 à raison d'un million de nouveaux habitants par décennie. La population de l'archipel a franchi la barre des 7 millions d'habitants en 2010. 
La réflexion aéroportuaire s'inscrit alors dans le cadre de la planification spatiale dont le rapport Abercrombie de 1948 forme un jalon important $\mathrm{t}^{5}$. En parallèle du redéveloppement de l'aéroport de Kai Tak s'engage la recherche de sites alternatifs. La première étude complète de relocalisation de l'aéroport est réalisée en 1973. Treize localisations sont étudiées parmi lesquelles le gouvernement retient la plus excentrée, Chek Lap Kok, îlot vierge de toute urbanisation situé à l'ouest de l'île de Hong Kong, face au delta de la Rivière des Perles entre Shenzhen et Macao sur l'autre rive. Le site constitue une opportunité inédite de développement, ouvrant la possibilité d'un déplacement du centre de gravité de la métropole vers le sud-ouest et l'embouchure du delta. Le choc pétrolier et la crise qui s'ensuit suspendent momentanément l'ambition des autorités. L'incertitude quant au futur politique de l'archipel joue également un rôle alors qu'une restructuration s'engage à la faveur de l'ouverture de la Chine aux capitaux extérieurs et de la création en 1979, de la Zone économique spéciale de Shenzhen, vers laquelle se délocalisent les activités manufacturières de Hong Kong.

Avec l' "Open door policy » engagée par la Chine s'annonce l'acte géopolitique qui va marquer le destin à venir de la colonie britannique bientôt cent cinquantenaire. L'accord de rétrocession à l'échéance du $1^{\text {er }}$ juillet 1997 fait l'objet d'une déclaration conjointe signée à Pékin le 19 décembre 1984 par les Premiers ministres Margaret Thatcher et Zhao Ziyang. Elle stabilise la situation politique de l'archipel pour une durée de 50 ans qui court au-delà de 1997. Hong Kong aura un statut spécial de région administrative, lui permettant de bénéficier d'une relative autonomie. Approuvé simultanément, un cadre de planification stratégique, la Territorial Development Strategy, fixe les ambitions de modernisation de l'archipel, poursuivant les logiques à l'œuvre qui gouvernent la cité (Kam Ng, 1993) ${ }^{6}$. À la fois libéral et autocratique, l'urbanisme de Hong Kong est l'héritier de son histoire doublement insulaire, géographiquement et politiquement. L'espace, façonné par un paysage maritime et montagneux, est exigu ; à peine $20 \%$ du territoire est urbanisé. Les caractéristiques topographiques de l'archipel

Pour une histoire de l'aménagement de Hong Kong, cf. Pryor et Pau (1993).

6 " Hong Kong Territorial Development Strategy, A Land-Use-Transport Strategy for the Growth of Hong Kong in the 1990s - Preliminary Submission », mai 1984 (source : Hong Kong University Library - HKUL). 
expliquent le mode de gestion quasi financière du sol comme actif dont le gouvernement a la propriété. Le sol est régi par un dispositif de baux emphytéotiques dont les ventes, limitées par des quotas annuels, se font aux enchères. Propriétaire terrestre et maritime, le gouvernement de Hong Kong contrôle également l'autorité portuaire ainsi que la Hong Kong Housing Authority et la Mass Transit Railway Corporation (MTRC), qui sont les opérateurs principaux du logement public et des réseaux de transports collectifs. Grâce aux land-use-transport policies, la planification urbaine favorise l'intégration étroite des réseaux de transport et des densités urbaines. Enfin, du fait de sa rente foncière, l'État noue des intérêts étroits avec les groupes immobiliers hongkongais qui contrôlent la majeure partie de la production urbaine privée sur le territoire de l'archipel.

Parallèlement aux démarches de planification, la sphère entrepreneuriale entre en scène. À la tête d'un consortium, Gordon Wu, président du groupe de travaux publics Hopewell Holdings Limited, défend à l'automne 1986 une alternative de relocalisation de l'aéroport dans la partie sud-ouest du port ${ }^{7}$. Cette proposition vient appuyer et contester en même temps le projet du gouvernement, qui défend également les perspectives de restructuration du port, tout en privilégiant le site de Chek Lap Kok. Ce dernier recueille l'approbation finale du gouvernement qui propose le 11 octobre 1989, à la séance de rentrée du Legislative Council, la création du nouvel aéroport, la mise en place d'une autorité aéroportuaire provisoire et l'implémentation d'un programme de développement dont l'achèvement est programmé à l'horizon de la rétrocession.

\section{Naissance d'une controverse}

Cette rentrée législative est marquée par la tragédie de Tiananmen et la répression violente des 3 et 4 juin qui a fait plusieurs centaines de morts, des milliers de blessés et autant d'arrestations brutales sonnant le glas d'un espoir de normalisation politique en Chine. La tension est

Ce projet dénommé « Western Harbour-Lantau Strategic Development " prévoit un programme de construction principalement réalisé par le secteur privé et estimé à 25 milliards de HK Dollars (soit 3 milliards de US Dollars). 
palpable entre les gouvernements britannique et chinois, les rencontres prévues à l'été autour des conditions de la rétrocession, ayant été suspendues à l'initiative de Margaret Thatcher.

Le discours de politique générale que prononce le gouverneur britannique David Wilson développe la stratégie assignée au futur de Hong Kong ${ }^{8}$. Il projette «l'infrastructure physique » dont l'aéroport est le fer de lance. S'égrène la liste des réalisations projetées : ligne de métro express et autoroute pour desservir l'aéroport, ville nouvelle à proximité, extensions territoriales sur la mer, redéveloppement de l'ancien site aéroportuaire. Il s'agit du plus grand programme de travaux jamais entrepris par la cité qui matérialisera le projet visionnaire de l'archipel, comme l'évoque en conclusion le gouverneur. "We have a clear vision of what we are trying to achieve. It is a vision that I hope will sustain Hong Kong during the present period of uncertainty and give us all confidence in our ability to overcome whatever problems confront us ${ }^{9}$. "

Deux mois plus tard est publié le projet final ${ }^{10}$. L'échéance de la rétrocession fixe le compte à rebours. Toutefois, l'accélération dont témoigne le processus de décision et la médiatisation dont il bénéficie ne doivent pas occulter les rapports de force qui s'expriment à la faveur de cette annonce. Les contestations sont de trois ordres. Politique d'abord, la Chine exprimant ses réserves face à un projet décidé sans son aval, qui risque selon elle de grever les finances de Hong Kong bien au-delà de l'échéance de 1997 alors que les Britanniques auront quitté l'archipel. Économique ensuite, les acteurs privés se mobilisant sous la houlette de Gordon Wu pour dénoncer un programme jugé onéreux. Les points de désaccord portent sur la localisation retenue, la nature et les tracés des infrastructures projetées, le principe de la ligne de métro express qui relierait l'aéroport à l'hypercentre, l'ampleur des réclamations (ou claims), ces nouveaux terrains qui seraient gagnés sur la mer (Leung,

8 "The Governor's Annual Address to the Legislative Council on 11 October 1989 ", Hong Kong Government Printer (source : HKUL).

9 "A Vision of the Future ", ibid, par. 99.

10 Hong Kong's Port and Airport Development Strategy, Final Report, décembre 1989. 
1991, p. 67-68) ${ }^{11}$. "We do have the choice! », argumente Wu qui ajoute : "Never has a project so large which involved so much been run by so few $^{12}$. » Pragmatiques ou opportunistes, les entrepreneurs n’hésitent pas à s'appuyer sur l'indélicatesse provoquée par le passage en force vis-à-vis de la Chine, pour mettre en défaut le gouvernement et avancer leurs pions en défendant un autre scénario qui, selon eux, pourrait résoudre la controverse.

Plus critiques encore, des experts soulignent deux défauts majeurs de la trajectoire que risque d'emprunter Hong Kong. D'une part, les infrastructures projetées ne sauraient à elles seules constituer la stratégie économique de la cité, confrontée à l'arrivée des High Tech. D’autre part, l'éveil des métropoles du delta - Shenzhen, Guangzhou, Macau ne peut être ignoré et nécessite de penser la coopération régionale à cette échelle. La critique cible de fait un projet qui privilégie la réalisation d'infrastructures lourdes, dont les liens avec les questions économiques et urbaines paraissent ténus (Kam Ng, 1993, p. 302-308).

Contestation publique enfin, car l'échéance de 1997 a mobilisé la création de groupements qui reprochent au gouvernement d'avoir négocié unilatéralement les conditions de la rétrocession et craignent une réduction des libertés politiques d'expression auxquelles les Hongkongais sont très attachés. Le projet aéroportuaire traduit ce climat de défiance. À l'origine bien perçu par ceux qui voyaient là l'occasion pour la cité de s'émanciper, il fait désormais l'objet de critiques vives ciblant les " bureaucrates » et les politiques qui développent le projet à huis clos.

Annoncée dans une période de glaciation des relations diplomatiques entre la Grande-Bretagne et la Chine, la décision portée par le gouverneur de Hong Kong de prendre le destin de la cité à pleines mains se comprend dans le contexte tendu d'un bras de fer entre les pouvoirs en présence, alors que monte la parole de la société civile. Tandis que s'engagent des pourparlers entre la Grande-

11 Hong Kong Government, " Review of Gordon Wu report on Port and Airport Development ", 2 octobre 1990 (source : HKUL).

12 "Airport alternatives: We do have a choice! ", Public Seminar, organized by the Study Group for Infrastructure Development, 25 mai 1991, Hotel Conrad (source : HKUL). 
Bretagne et la Chine, s'amorcent les préparatifs du chantier prenant de court la République populaire qui redouble de menaces. À l'issue de plusieurs tours de négociation, un compromis finit par s'établir à l'été 1991, signé le 3 septembre entre les Premiers ministres John Major et Li Peng, qui acte un partage des risques entre le gouvernement de Hong Kong et la Chine ${ }^{13}$. Surtout, John Major s'engage à se rendre en Chine, premier des Européens de l'Ouest à le faire après les événements de Tiananmen. Cette conclusion ne contribue pas à rassurer les Hongkongais qui voient dans cet accord une concession trop facile faite à la Chine, lui conférant un pouvoir excessif sur les finances de Hong Kong après 1997.

\section{La mise en récit}

La controverse fait rentrer sur la scène les protagonistes d'une intrigue qui va durer plusieurs années : acteurs politiques et autorités gouvernementales, entrepreneurs privés, hommes de l'art, groupements citoyens, sur fond de négociations entre la Chine et la Grande-Bretagne, le tout étant largement couvert par la presse. Entre l'automne 1989, date à laquelle s'officialise l'annonce du déplacement du nouvel aéroport, et l'été 1998, date de son ouverture, les archives du South China Morning Post, quotidien anglophone le plus lu de la région, témoignent de plusieurs milliers d'occurrences du sujet aéroportuaire.

Loin de faire l'unanimité, le chantier qui s'amorce voit s'ouvrir une période intense de négociations et de reformulations du projet. Défenseurs et pourfendeurs du projet vont être mobilisés autour d'une réorientation du dessein métropolitain, dont l'énoncé va s'étoffer au fur et à mesure de l'élaboration de la stratégie. Publiée en octobre 1991, la nouvelle version de la Port and Airport Development Strategy (PADS), qui s'orne d'un sous-titre "A foundation for growth ", résulte de cette période active qui voit les arguments du projet évoluer.

13 " Memorandum of understanding concerning the construction of the new airport in Hong Kong and related questions », p. 1, Project, 4 juillet 1991 (source : HKUL). 
Figure 1. Photographie de la maquette du projet d'ensemble figurant dans la Port and Airport Development Strategy, 1991. Au sud-ouest, on distingue les contours du futur aéroport de Chek Lap Kok, adossé à lîle de Lantau.

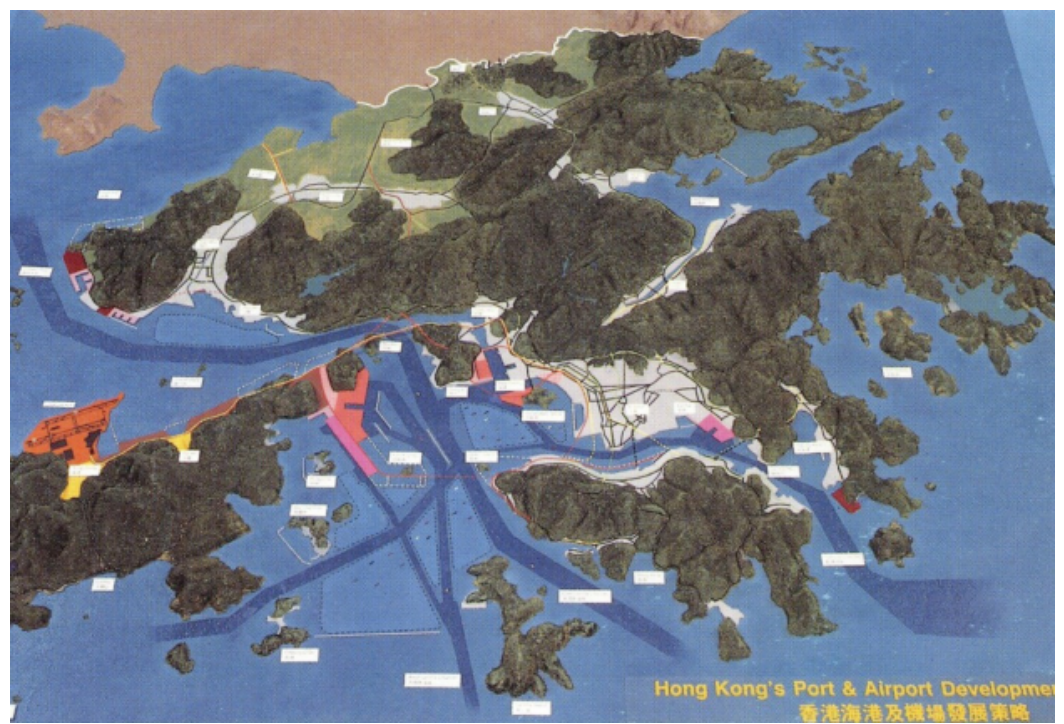

Source : Hong Kong Government.

"Following the 1989 decision on Port and Airport Development Strategy, steps were immediately taken to create an organisational structure in government to provide a means by which broad planning concepts would be translated into projects ${ }^{14}$. " Si le gouvernement reste ferme sur le choix du site, il n'en demeure pas moins qu'il ne peut se passer de la sphère économique pour faire réussir son projet. Celle-ci constitue en effet le moteur essentiel de la croissance du territoire. Revenant sur l'histoire urbaine de Hong Kong, la deuxième édition de la PADS inscrit le projet comme nouveau terrain d'exercice de la coalition forgée autour du gouvernement et de la sphère entrepreneuriale. "The community as a whole has the necessary resourcefulness energy, institutional structures and professional capabilities to turn visions into viable projects which,

14 "Setting the scene ", Hong Kong's Port and Airport Development Strategy, op. cit., p. 66. 
in turn, will create another platform for growth ${ }^{15}$. " Le gouvernement rejoint ici les revendications des acteurs économiques qui défendent l'idée selon laquelle l'infrastructure doit être un projet total intégrant le développement du port et de la métropole.

Cette stratégie se déploie à plusieurs échelles. Laéroport sera d'abord la pièce maîtresse du développement régional de Hong Kong, s’inscrivant comme la proue de son avancée dans le delta de la Rivière des Perles et au-delà en Chine du Sud. Le gouvernement consolide ensuite le socle territorial du projet. Face aux critiques sur sa dimension par trop infrastructurelle, oublieuse des considérations urbaines, sociales et économiques de l'archipel, la stratégie convoque l'ensemble des réflexions qui ont nourri son projet ${ }^{16}$. Le premier acte de la fondation va se traduire par la mise au diapason des plans et des programmes. L'accord scellant les négociations entre la GrandeBretagne et la Chine marque le lancement du programme de la réalisation du nouvel aéroport, l'Airport Core Programme. Estimé à 200 milliards de dollars hongkongais (soit 25 milliards de dollars américains), il projette la construction d'un ensemble de grands ouvrages composant la future infrastructure de l'archipel ainsi que la viabilisation de nouveaux territoires, arrimés à elle, qui formeront des lieux de développement futur de la métropole (Kam Ng, 1993, p. 300-302, 309) ${ }^{17}$. Actualisée en 1988, la nouvelle édition de la Territorial Development Strategy a nourri les orientations régionales et territoriales du projet aéroportuaire, tandis que le Metroplan, qui se prépare depuis 1987, est approuvé en septembre 1991. Document à portée stratégique et juridique, il planifie le desserrement de la métropole, par l'expansion des réseaux de transport en commun et des villes nouvelles dans les nouveaux territoires, ainsi que par la déconcentration du centre grâce à la création de nouveaux claims $^{18}$.

Les plans nourrissent les programmes; ils en procèdent en même temps, la décision aéroportuaire constituant l'acte de mise en ordre de bataille de l'ensemble du dispositif de planification. Pour s'ancrer

\footnotetext{
15 «Future Horizons ", ibid., p. 87.

16 Ibid., p. 42.

17 Hong Kong Government, Hong Kong Airport Core Programme, 1991 (source : HKUL). Le plan de financement sera négocié et conclu 4 ans plus tard. Le montant estimé sera ramené, à l'issue de sa réalisation, à 160 milliards de HK Dollars (soit 20 milliards de US Dollars).

18 Metroplan. The selected strategy, septembre 1991 (Fouchier, 1993).
} 
territorialement, le projet infrastructurel s'appuie sur les idées en gestation, les confrontant, déclenchant leur mise en œuvre tout en les assujettissant à sa propre logique. Les deux années qui séparent la publication des deux versions, initiale et définitive, de la PADS, constituent une période intense de production de discours d'ordres différents, politiques, techniques, urbains, journalistiques, universitaires. La publicisation du projet, au travers de la controverse qu'il suscite, contribue à faire advenir la réalité de l'événement, à l'imposer à tous.

\section{La création d'un territoire}

Pendant que les politiques occupent la tribune, se monte le théâtre des opérations avec l'entrée en scène des concepteurs et des bâtisseurs, et un nouvel acte spectaculaire qui s'engage, celui de la création du sol de l'aéroport. " Coming to Chek Lap Kok was a little like going into a desert environment where you take over an area and mould it to meet your needs ${ }^{19}$. " En écho à ses souvenirs, le directeur des travaux de l'autorité aéroportuaire provisoire souligne le caractère pionnier du chantier de l'aéroport dont la conception architecturale est confiée à Norman Foster, auteur du siège de la Hong Kong and Shanghai Bank of China, devenu l'un des fleurons architecturaux de la ville. L'île de Chek Lap Kok est arasée, agrandie et réunie à l'îlot montagneux de Lam Chau, également arasé, figurant au total un territoire de 1250 hectares, soit quatre fois la superficie originelle de l'île. La terre servant au remblaiement est récupérée de la destruction des reliefs et consolidée par le dragage des fonds marins. D'autres travaux d'envergure s'engagent parallèlement, conformément au programme édicté par le gouvernement. Deux ponts suspendus et un viaduc, tous trois ferroviaires et routiers, sont édifiés permettant sur une distance de 2200 mètres le franchissement de la baie dans sa partie ouest. Trois nouvelles autoroutes sont construites ainsi qu'un tunnel d'une longueur d'un mile passant sous le port.

"The world of Chek Lap Kok " : les superlatifs sont nombreux à souligner la dimension titanesque du chantier aéroportuaire, qualifié de plus grand au monde. À commencer par l'arasement des montagnes

19 Hong Kong Airport Authority, Hong Kong International Airport. Vision to Reality, In total harmony, 1998, p. 123. 
et les terrassements qui charrient des volumes impressionnants de matières, avec ce chiffre de 10 tonnes de matière par seconde au point le plus intense du chantier qui sera cité à plusieurs reprises. Des parallèles dimensionnels sont brandis, pour tenter de ramener l'échelle de la réalisation à des références connues. Les métaphores redoublent le caractère extraordinaire de l'entreprise : «Work on the Chek Lap Kok airport, the world's largest construction project, began by moving a mountain. Since then, airport authorities have been bulldorizing world records aside at a steady pace. The terminal building will be the world's largest, built in a total of 13 million mandays "(Graham, 1998). Le chantier lui-même est comparé à une ville, immense ruche qui réunit à son moment le plus fort, près de 20000 personnes, et dont le panel de nationalités présentes l'apparente à une Babel en construction.

La création du territoire de l'aéroport s'accompagne de la construction de l'Airport Express Line, qui dessert la cité hongkongaise en plusieurs lieux, à Central sur l'île de Hong Kong, et Kowloon sur la péninsule chinoise, là où seront édifiés deux hubs de transport, qui accueilleront chacun une aérogare in town. Celle-ci doit permettre aux passagers aériens quittant la ville d'accomplir toutes les formalités d'enregistrement avant de rejoindre la plateforme aéroportuaire pour embarquer dans leur avion, les bagages enregistrés en ville étant acheminés dans l'un des wagons réservés du train de l'Airport Express Line à destination de l'aéroport. Avec ses gares, la ligne de métro forme un dispositif qui s'étend sur les 34 kilomètres séparant l'aéroport de l'hypercentre, "à 23 minutes " seulement. Selon les autorités, près de la moitié des passagers aériens devraient emprunter cette ligne qui doit contribuer à la réussite du projet aéroportuaire ${ }^{20}$. Pour la MTRC qui réalise la ligne, le chantier constitue une nouvelle occasion de consolider son empire. En l'espace d'une décennie, elle va d'ailleurs devenir le principal aménageur de la cité. L'autofinancement de la ligne a en effet été assuré grâce au principe de densification autour des gares édicté par le Metroplan, qui a permis à la compagnie de réaliser, en contractualisant avec des promoteurs, des ensembles urbains de grande ampleur ${ }^{21}$.

20 L'Airport Express Line ne sera finalement utilisée que par $15 \%$ des passagers (Hirsh, 2016, p. 4).

21 À propos du rôle de la MTRC comme aménageur urbain, cf. Tiry (2003). En 2011, MTR disposait d'un patrimoine immobilier de $750000 \mathrm{~m}^{2}$ d'espaces commerciaux 
Toutefois, l'accélération des chantiers ne se fait pas sans accrocs. Le déploiement de l'Airport Core Program cristallise une nouvelle controverse autour du futur de la baie. La superficie des claims, ces extensions sur la mer qu'a planifiées le Metroplan et que réalisent les travaux programmés, fait l'objet d'un vif débat opposant ceux qui comptaient profiter du sol gagné sur la mer pour dégager de nouvelles ressources foncières, à ceux qui entendent sauvegarder le patrimoine urbain et naturel de la baie. Avec la réutilisation des terrains libérés par l'ancien aéroport Kai Tak dont il est proposé d'étendre la surface, c'est plus d'un millier d'hectares de sol que les autorités envisagent de créer, constituant un trésor foncier d'autant plus rentable qu'il serait bien desservi par les nouveaux réseaux de transport. Ce projet rencontre l'opposition farouche des citoyens qui se mobilisent au travers d'une campagne "Save our harbour " portée par la Society for Protection of the Harbour et relayée par les associations professionnelles d'architectes, d'urbanistes et d'ingénieurs, ainsi que par des universitaires (Loh, 2001). Tandis que se réalisent les travaux d'infrastructure au large de Chek Lap Kok, la campagne est menée tambour battant, ciblant des méthodes d'urbanisme dépassées. Elle s'achève en 1998 sur un retrait des projets initiaux en faveur de propositions plus mesurées. Les opposants saluent la décision : "As an update, there has been a remarkable change in attitude by the Government over the past year ${ }^{22}$. "Si les autorités de Hong Kong ont renoncé à leur projet d'origine, la controverse se clôt toutefois sur une perspective ambitieuse d'extension territoriale.

\section{La médiatisation de l'événement}

"Today, if you want an airport to be a gateway to a city, it has to make a statement, to act as a symbol and it has to work ${ }^{23}$." Largement relayée par les médias, l'ouverture de l'aéroport constitue un événement historique, ce retentissement faisant écho à l'acte politique

et tertiaires, 80000 logements. Source : "Moving Experience. The MTR's first 36 years ", South China Morning Post, 2011, p. 97.

22 Society for the Protection of the Harbour, Letter to The South China Morning Post, 18 septembre 1999, citée in Christine Loh, ibid., p. 71.

23 Souligné par le directeur du projet aéroportuaire Winston Shu pour l'agence de Norman Foster, in Vision to Reality, op. cit., p. 73. 
de la rétrocession qui a eu lieu un an avant. Comme pour marquer symboliquement l'arrimage en ville du nouvel aéroport, c'est l'aérogare in town de Hong Kong qui ouvre la première, le 21 juin 1998, à l'issue de 4 ans de travaux. Sous les auspices de la MTRC, elle bénéficie d'une inauguration fastueuse, à l'occasion de laquelle le président de la compagnie de métro réaffirme la puissance de son entreprise, devenue l'un des plus grands acteurs urbains de la ville. Le 2 juillet, c'est au tour du nouvel aéroport d'être inauguré par le président chinois. "Vision has become a reality » : en présence de 3000 invités, Jiang Zemin célèbre l'événement comme l'accomplissement d'un rêve ${ }^{24}$. Le 6 juillet, jour de l'ouverture officielle de l'aéroport, acte enfin la mise en service de l'Airport Express Line. Cette concomitance des dates n'est nullement le fruit du hasard. Aux yeux des autorités, les trois ensembles doivent être mis en service conjointement pour marquer l'avènement de la nouvelle infrastructure.

Derrière ce triomphe célébré par les inaugurations, la réalité est toutefois moins lisse, comme l'exprime un article de Newsweek qui consacre sa couverture du 13 juillet à l'événement en l'illustrant d'un portrait de l'un des ouvriers ayant œuvré sur le chantier. "Hard times in Hong Kong after the handover : a bright new airport and a stalled economy »(Elliot, 1998) : pendant que s'activaient les chantiers qui allaient transformer Hong Kong, la crise asiatique a frappé de plein fouet la région et les grands travaux qui s'achèvent paraissent en décalage en regard des difficultés économiques que vit l'archipel. L'image brillante du succès se brouille et la question du futur se repose : "To survive hard times, the city will have to invent other ways to prove its worth" (ibid., p. 3). Tandis que la modernité des infrastructures exige d'avoir le public au rendez-vous, les critiques qui avaient fusé sur le risque d'un investissement en infrastructures peu souple resurgissent. Avec l'ouverture de l'aéroport s'inaugure une période au cours de laquelle la cité va devoir digérer des échelles inédites et s'adapter à des lendemains incertains.

24 "Hong Kong International Airport, Opening Ceremony », 2 juillet 1998 (source : HKUL). 


\section{Micromonde versus macromonde}

Le changement de périmètre opéré par le nouveau dispositif territorial est illustré par la photographie prise en l'an 2000 par les architectes de Map Office, d'un grand panneau institutionnel qui masque le chantier de l'International Finance Center. Situé sur l'île de Hong Kong, ce supercomplexe immobilier doit accueillir la première aérogare in town de la ville. L'image saisie figure un triptyque : à gauche, une vue oblique de la maquette du nouvel aéroport ouvert deux ans auparavant ; à droite, une vue de face du futur complexe, surmonté de deux gratte-ciel qui parachèvent le skyline de l'île; au milieu, une vue du métro rapide flottant dans les airs entre les deux vues. "The new Airport in Central » : le slogan qui s'affiche traduit la double réimportation au centre de l'aéroport périphérique (Gutierrez et Portefaix, 2000). D’une part, la ligne de métro permet de rapprocher l'aéroport du centre, la distance de temps (23 minutes) ayant supplanté la distance de lieu. D’autre part, l'aérogare in town maintient au centre les fonctions aéroportuaires principales en ne reléguant à l'extérieur que celles qui sont incompatibles avec un environnement dense. Les contours du dispositif infrastructurel sont actés autour de ses trois composantes indissociables : la plateforme ex urbis, la ligne de métro et l'aérogare in town.

Figure 2. "Fluid machine : 23 minutes de l'aéroport », 2000

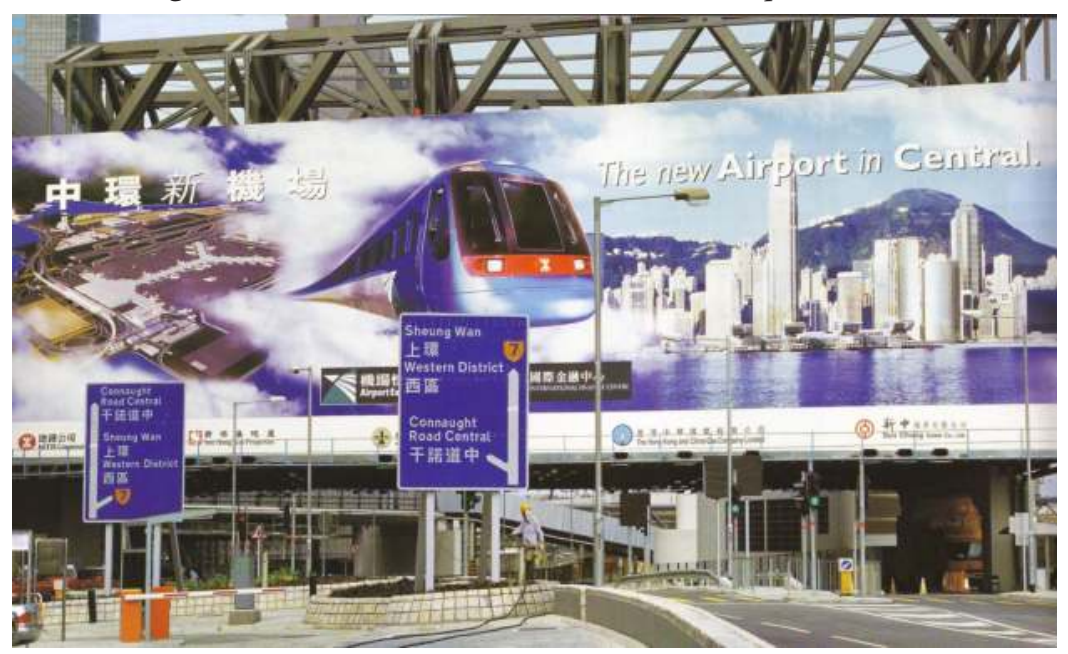

Source: Photographie Laurent Gutierrez et Valérie Portefaix, Map Office. 
L'édification du complexe immobilier de l'IFC témoigne d'une autre forme de distorsion des échelles. Pilotée par la MTRC, la construction du complexe est achevée en 2003, produisant un block unique d'une superficie inédite, près de 6 hectares, constitué d'un unique bâtimentpodium de 8 mètres de haut surmonté de deux tours. Intégrée au complexe, l'aérogare in town, aux matériaux sobres - pierre, aluminium, verre -, dispose d'une façade qui donne sur une esplanade. Bâtiment-socle de l'échelle de plusieurs îlots, le podium est un dispositif constitutif de l'urbanisme hongkongais. Parc ou place, sa toiture peut être publique, reliée par des passerelles aux réseaux viaires de la ville et aux autres bâtimentspodiums. C'est le cas pour le complexe de l'IFC, dont le toit offre une vue imprenable sur le paysage de la baie. Un système de passerelles aériennes, la Central Elevated Walkway, ouverte 24 heures sur 24, relie le superblock à la canopée urbaine existante formée par les circulations piétonnes de l'île (Tiry, 2009). La mixité des fonctions en fait un espace urbain total que le concepteur de la station de transport, Rocco Yim, décrit comme " a complex matrix of transport, public and commercial functions that is in itself a mini-city within a city (Rocco, 2002).

En face de l'IFC s'érige, comme une ombre projetée sur la péninsule de Kowloon, un autre superblock, West Kowloon, plus imposant encore, qui accueille la deuxième aérogare in town. Achevé en 2010, déployé sur une superficie de 14 hectares, l'ensemble compose un bâtimentpodium de 18 mètres de haut coiffé d'une dalle surplombée de 16 tours de logements, hôtels et bureaux, et sur laquelle sont aménagés espaces extérieurs, jardins et plans d'eau. Dénommée ICC (International Commerce Center), la plus haute tour a dépassé celle de IFC qui s'élève en face sur l'île de Hong Kong, formant avec elle les deux colonnes d'une porte imaginaire qui cadre l'entrée de la baie. Conçue par l'agence britannique Terry Farrell Partners ${ }^{25}$ et réalisée par la MTRC, l'opération défie les records de construction atteints jusqu'à présent dans l'archipel. À commencer par le terrain sur lequel elle s'édifie, plus grand projet de création de sol jamais réalisé à Hong Kong. La compagnie MTRC avouera qu'elle n'est pas sûre de revoir cette échelle de développement. Le long du superblock passe une autoroute de deux fois six voies qui relie

$25 \quad$ L'agence britannique de Terry Farrell a créé son bureau de Hong Kong en 1991. Terry Farrell est l'auteur du Peak Tower, un autre landmark du paysage de Hong Kong. 
l'aéroport et l'île de Lantau à la ville. Au-delà devraient se réaliser, côté baie, un parc, des quartiers résidentiels et un ensemble de musées.

À l'intérieur du complexe de West Kowloon, le hub de transports qui accueille l'aérogare in town se déploie sur une surface de 220000 mètres carrés, l'équivalent d'une grande aérogare, dont les codes et les fonctions sont à son image. La signalétique donne le ton : " departure ", " arrivals ", " transfer ", " city links ». La maîtrise d'œuvre est particulièrement active sur le front de la rhétorique. Transport Super City : c'est le titre de l'ouvrage que publie l'agence Farrell à l'occasion de l'inauguration de l'Airport Express Line. Sa préface est signée du président de la MTRC qui énonce : «In effect, we have brought our new airport back to the city. » Entendant " urbaniser l'aéroport », Farrell enchaîne quelques pages plus loin, se mettant dans les pas de Norman Foster à qui il emprunte l'idée d'un petit diagramme qui figure le passage de "Airport as city " vers "City as airport ", indiquant par là que c'est désormais la ville entière, incarnée dans le complexe, qui est devenue un terminal aéroportuaire (Farrell, 1998, p. 7-8).

\section{Image versus usage}

Plus qu'une ville autosuffisante, c'est l'image d'une autre ville qui s'esquisse. Au travers des vues que scénarisent les tracés des voies ou l'aménagement des gares, la ligne offre d'abord à voir dans un seul mouvement cinétique, un préambule narratif racontant la ville qui advient. Vu du train, le travelling dévoile le skyline montagneux, maritime, portuaire et urbain de la métropole avec ses jeux d'accélération et de ralentissement, ses mouvements de courbes, de plongeons et de réapparition. Vu du sol, il s'appuie sur la continuité de l'aménagement, du traitement architectural des intérieurs, l'aéroport appartenant à l'identité de la ville, tandis que les gares de centre-ville appartiennent à l'espace de l'aéroport. La ville « selfcontained ", où la distinction entre réel et spectacle se brouille, impose ici une scénographie de la ville comme expérience visuelle et sensitive.

Dans le panorama qu'elle donne à voir, dans la séquence active qu'elle fait vivre, dans les formes qu'elle décrit, l'infrastructure met la ville en narration, conduisant à faire du dispositif aéroportuaire une ville en soi tandis que la ville se pratique comme un aéroport. C'est ce que souligne Naonori Matsuda dans l'un des premiers essais analytiques consacrés à la " ville-aéroport » de Hong Kong, publié en 1997. "If the boundary 
between the city and the airport were dissolved, the entire city may then be regarded as an airport itself [while] the new transport infrastructure integrates the existing city into an airport" (Matsuda, 1997, p. 77, 81).

Figure 3. Accès à l'aérogare urbaine de West Kowloon, Hong Kong, 2017

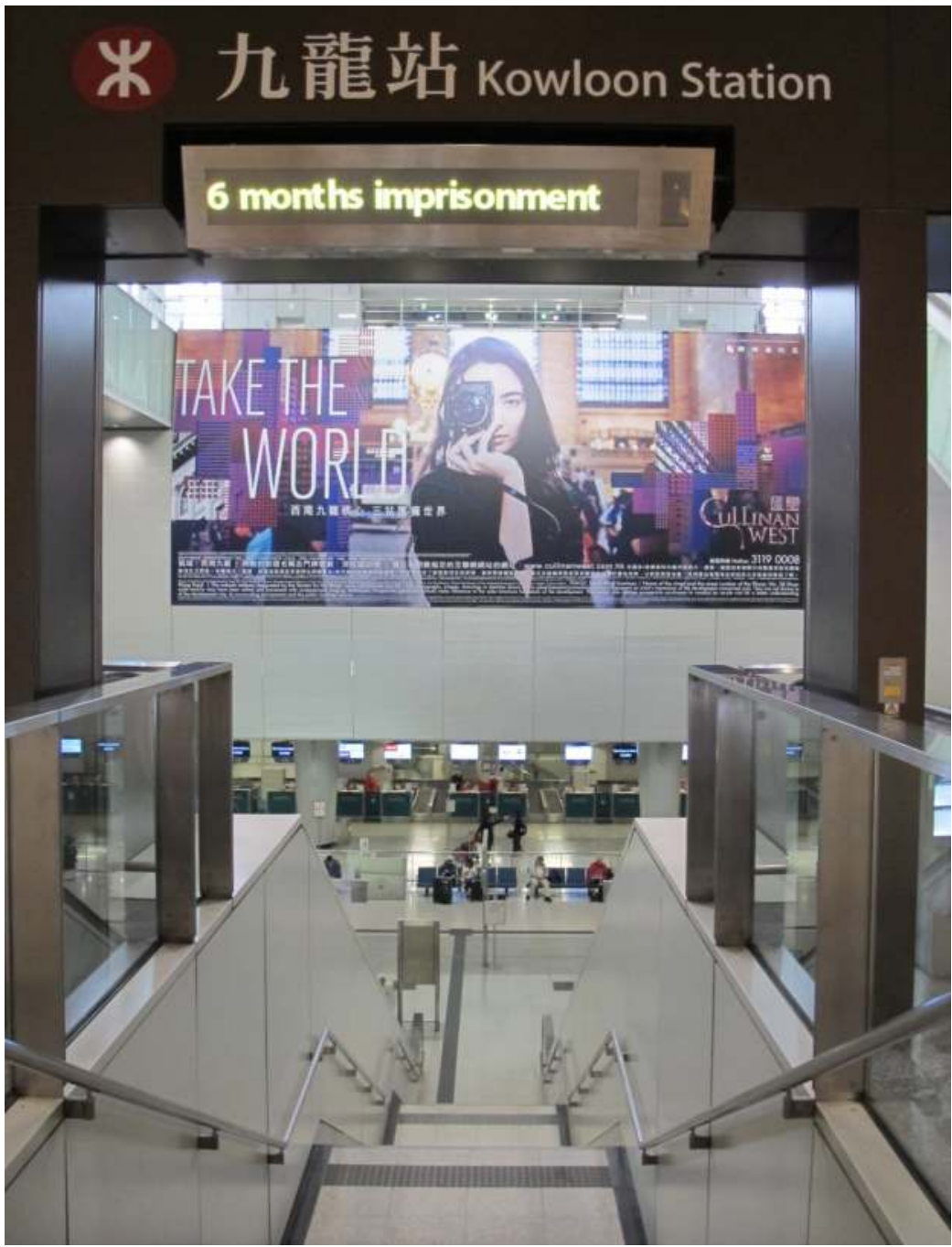

Source: Photographie Nathalie Roseau, mars 2017. 
Cette mise en image se confronte à la pratique, ce décalage entre la fiction et l'expérience se lisant à plusieurs échelles. Celle du temps d'abord qui n'a pas la même valeur, selon qu'il est affiché dans des slogans tels que celui martelé par les autorités - " 24': the fastest link to the city $\rrbracket^{26}$ - ou pratiqué par des voyageurs qui souhaitent rejoindre leur hôtel ou leur domicile. Le raccourcissement sur la carte des distances de temps que parcourent les réseaux doit être mis en regard de l'agrandissement sur le plan des distances métriques internes aux différents composants de la ville, comme celles que l'on pratique à Hong Kong Station ou West Kowloon au sein desquels les correspondances figurent déjà un déplacement en soi.

Celle de l'habiter ensuite. Car cette ville que l'on projette est une ville de l'élite où peu vivent. La "ville aéroportuaire " - qu'elle soit horizontale, celle qui relie l'aéroport à l'hypercentre, ou verticale, celle des superblocks - est une ville accessible aux classes supérieures et aux touristes, où se confrontent la mondialisation des opulents et celle des plus modestes qui la font tourner sans jamais la consommer. En dépit de l'énoncé complet des composants programmatiques qui la qualifient de ville sur le papier, le parcours de la cité qui prend forme sur le claim de Kowloon produit une sensation étrange de vide urbain.

Celle de l'usage enfin, car la ville projetée est avant tout un mirage. Si le changement de cap s'adresse aux happy few de la mondialisation, qu'en est-il de la majorité qui forme la ville, des classes moyennes aux plus pauvres qui contribuent pleinement à la mondialisation hongkongaise ? Des centaines de milliers de citoyens philippins et indonésiens, employés domestiques de l'archipel - une grande partie sont des femmes - recourent chaque année aux moyens de transport aériens pour revenir dans leurs pays y retrouver leurs familles. Mais peu fréquentent les lieux que nous décrivons. En effet, avec les low cost aériennes qui se sont multipliées en Asie du Sud-Est, s'est développé à Hong Kong un système terrestre low cost, comme l'atteste la noria des

${ }^{26}$ Entre-temps, la compagnie de métro a revu (légèrement) à la hausse la distance de temps séparant l'aéroport de l'hypercentre. Le registre des slogans reste cependant inchangé. 
navettes par bus qui relient la cité à l'aéroport, en offrant un parcours alternatif à celui de l'Airport Express Line à un prix cinq fois inférieur (Hirsh, 2016).

\section{De la métropole à la région, la course infrastructurelle}

"We therefore need to ensure that Hong Kong can keep up with the "globalism" it represents. That implies constantly moving forward $[\ldots]^{27}$. " Comment poursuivre lorsque, pendant près de 10 ans, la ville a vécu au rythme d'une course fulgurante ? Comme l'énonce la nouvelle vision stratégique produite en 2007 , la cité hongkongaise ne semble pas vouloir s'interrompre. Une fois l'événement aéroportuaire advenu, quels nouveaux récits s'amorcent?

Les documents-fictions qui avaient couronné l'achèvement du programme de l'Airport Core Programme inscrivaient les réalisations dans la genèse longue et le tournant accéléré dont l'infrastructure aérienne de l'archipel faisait l'objet. Rétrospective, l'histoire que retraçaient ces documents était aussi prospective. "The new Hong Kong International Airport has opened new horizons and once again, the city of 6.5 millions people is productively mapping its future in a brave new world ${ }^{28}$." "Mapping the future " : le futur était encore à l'œuvre, soit pour en exprimer l'histoire soit pour en renouveler le projet. Ce discours faisait écho au récit mégalopolitain esquissé deux ans plus tôt par David Li Kwok Po, membre du Legislative Council:

"Imagine, it is the year 2006. Pearl City: one of the largest, richest urban areas in the world. Including its suburbs, it spans more than $160 \mathrm{~km}$ from north to south. Pearl City has a population of more than 40 million [...]. Pearl City's infrastructure is the envy of the world. Mass transit rail systems and a network of highways, bridges and tunnels enable residents to travel from Stanley Market [in Hong Kong] to Guangzhou city centre in less than 90 minutes " $(\mathrm{Li}, 1996)$.

\footnotetext{
27 "Hong Kong's evolving role in Greater PRD region ", Hong Kong 2030, Planning vision and strategy, Final report, 2007, p. 50-51. 
Figure 4. "Pearl City, Here We Come",

illustration de l'article du South China Morning Post, 6 juillet 1996

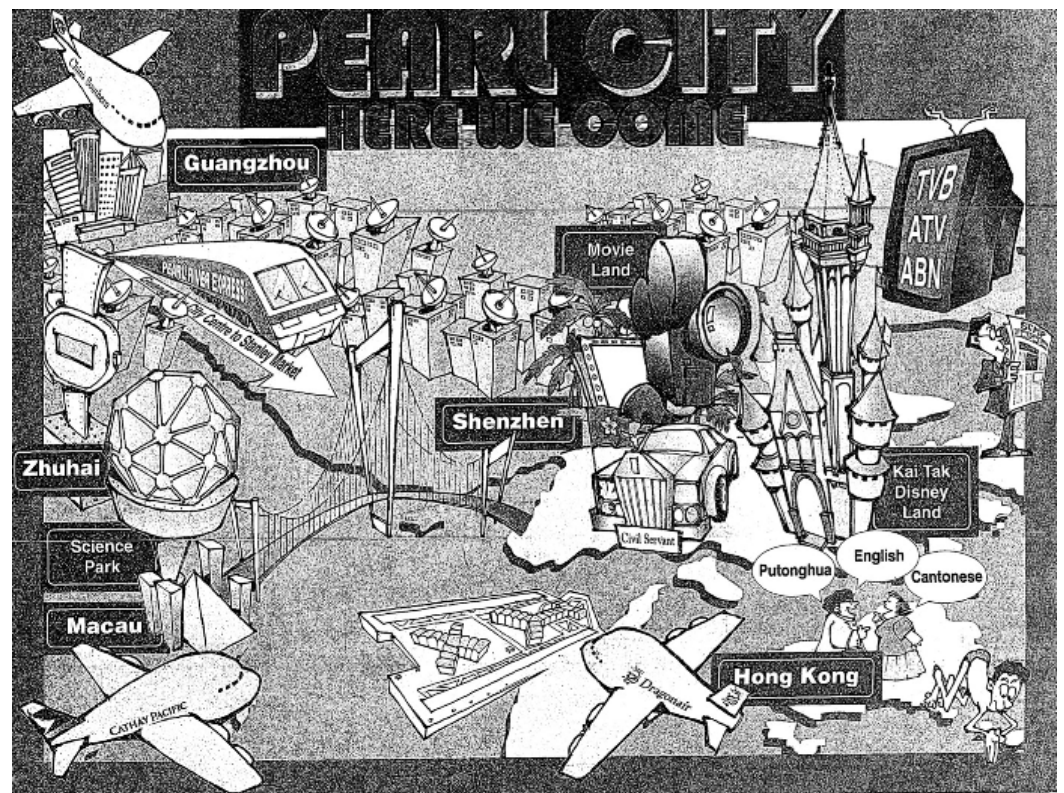

Source: South China Morning Post, 6 juillet 1996.

Cette prophétie s'est depuis lors réalisée. Derrière le chantier de l'infrastructure aérienne hongkongaise, c'est en effet une transformation d'envergure qui a façonné la région du delta de la Rivière des Perles, aujourd'hui forte de 10 métropoles millionnaires. Les réseaux autoroutiers se sont densifiés, raccourcissant les distances de temps, franchissant les obstacles naturels. Le système aéroportuaire a connu aussi de profondes mutations. En 1992, Kai Tak avait un quasimonopole régional. Pour se rendre dans le delta et la Chine du Sud, la plupart des visiteurs internationaux transitaient par Hong Kong puis empruntaient le train au départ de Kowloon. Trois ans plus tard sont apparus trois nouveaux aéroports internationaux, à Shenzhen, Zhuhai et Macau, rejoints par la nouvelle plateforme de Chek Lap Kok (1998), puis la création de l'aéroport de Baiyun à Guangzhou (2004). De même que Hong Kong s'est modernisée simultanément au déploiement urbain de la région, le développement de son aéroport s'est réalisé en parallèle 
d'un mouvement plus large au sein duquel les infrastructures se sont additionnées et concurrencées, en même temps qu'elles impliquaient en permanence le réajustement des unes par rapport aux autres. C'est par le terme de coopétition que les autorités régionales désignent cet équilibre instable qui régit le système des grandes villes du delta et dont les infrastructures sont un marqueur significatif (Jianfa, 2010).

Cette course infrastructurelle se poursuit encore avec des chantiers d'ampleur. Le groupe immobilier Hopewell, dirigé par Gordon Wu, a ainsi ouvré à la construction du gigantesque pont autoroutier - long d'une quarantaine de kilomètres - qui reliera bientôt les métropoles des deux rives opposées du delta, Hong Kong à Zhuhai et $\mathrm{Macau}^{29}$. Le Hong Kong Express Rail devrait quant à lui bientôt relier Hong Kong à Guangzhou en 48 minutes et Shenzhen en 15 minutes à peine, raccourcissant encore les distances de temps au sein de la région ${ }^{30}$. Et l'aéroport prévoit encore de s'étendre sur la mer avec l'ajout d'une troisième piste, très controversée au regard des menaces qu'elle ferait peser sur l'écosystème marin.

\section{Pannes des récits, les horizons post-2047}

Pour impressionnant qu'il soit, le panorama des infrastructures réalisées ou projetées à l'échelle du delta métropolitain ne saurait à lui seul répondre à la question du futur urbain de Hong Kong et de sa région. "Vision without action is a daydream. Action without vision is a nightmare. " C'est par ce proverbe japonais que s'ouvre le rapport Hong Kong 2030, Planning vision and Strategy, produit en 2007, qui présente la nouvelle stratégie urbaine et nationale de Hong Kong. La question des relations entre les deux métropoles « miroir » de Shenzhen et de Hong Kong, y est largement abordée. Building a Hong KongShenzhen Metropolis : c'est aussi l'objet du rapport d'un think tank,

\footnotetext{
29 Succession de tunnels et de ponts reposant sur des îles artificielles, l'ouvrage placerait Hong Kong à 45 minutes de Macau (au lieu des 4 heures actuelles).

30 C'est d'ailleurs de la gare de West Kowloon que partiront les trains express vers la rive est du delta. Ces projets s'inscrivent dans la stratégie de mise en place des réseaux de grande vitesse ferroviaire en Chine, dont 20000 kilomètres sont déjà construits.
} 
le Bauhinia Foundation Research Center, publié la même année, qui étudie les différents possibles de l'« agrandissement " des contours de la métropole ${ }^{31}$.

Ces deux documents font l'aveu d'une difficulté première, celle de nommer ce qui advient. Alors qu'elle n'était qu'un village de pêcheurs il y a à peine 40 ans, Shenzhen est devenu l'un des champions métropolitains de la Chine du Sud, perfusé d'infrastructures ferroviaires, routières et aéroportuaires qui la rendent désormais incontournable. La ville a dépassé démographiquement et industriellement le port de Hong Kong, l'ensemble figurant un territoire bicéphale de plus de 20 millions d'habitants, séparé par une frontière dont l'urbanisation accroît la complexité politique, circulatoire, sociale. Démunies par l'absence de sémantique idoine, les visions publiées recourent à un vocabulaire de l'hyperbole pour tenter de nommer cet ensemble inédit : "Metropolis ", "Megalopolis ", "Megacity ", sans non plus que ces termes suffisent à définir un horizon viable. Car la question du « concept » métropolitain ne tient pas uniquement à la forme urbaine duale que composent les deux villes autonomes. Elle procède de la dimension politique que revêt l'enjeu de cet " agrandissement ". "Interface ", "Intégration ", "Assimilation": chaque notion fait écho à des enjeux politiques qui touchent à l'avenir de Hong Kong, alors qu'en 2047, après deux siècles d'indépendance, la ville-archipel reviendra définitivement dans le giron chinois.

Les projets infrastructurels initiés par la cité hongkongaise esquissent différents scénarios qui confortent son désir d'émancipation tout en cultivant l'ambiguïté de son statut politico-territorial. Former une métropole avec Shenzhen par la densification de ses liaisons terrestres rapides ? S'ouvrir à l'autre rive du delta par le franchissement de l'embouchure ? Se renforcer dans son développement endogène, en profondeur et en hauteur, alors que le paysage de la baie bruisse encore des dizaines de grues qui réalisent le chantier de West Kowloon? Comme cela avait été projeté lorsque le site de Chek Lap Kok avait été retenu, le point névralgique de ces développements se situe au cœur du territoire aéroportuaire, les liaisons infrastructurelles projetées en direction de

31 Bauhinia Foundation Research Center, Building a Hong Kong-Shenzhen Metropolis, Executive Summary, 2007. 
Shenzhen ou du delta, s'y entrecroisant avec des projets immobiliers d'envergure. Une île artificielle à l'est de Lantau, équipée de complexes touristiques et commerciaux, formerait ainsi l'un des premiers jalons urbains de l'ouvrage d'art qui relierait Hong Kong à Zhuhai. La ligne ferroviaire express qui relierait Hong Kong et Shenzhen serait quant à elle interconnectée à l'Airport Express Line au sein de la gare de West Kowloon. Enfin, une autre connexion ferroviaire rapide projetterait de réunir les deux plateformes aéroportuaires en 15 minutes seulement leur trafic cumulé atteignant alors 100 millions de passagers par an.

«Port and Airport Development Strategy, and Metroplan to a certain extent, propagates a city of infrastructure, which extends the scope of Hong Kong's vision of itself beyond the present border with China. The future development of the infrastructure may cause the physical boundary to become less clear" (Matsuda, 1997, p. 82). Comme le signalait dès 1997 Naonori Matsuda, la question des frontières entre Hong Kong et la Chine, de leur maintien et de leur dépassement, travaille le projet métropolitain, l'infrastructure les transcendant sans non plus les effacer. L'absence de récit post-2047 ou plutôt la présence d'un horizon (l'intégration dans un seul système régional) qui empêche l'élaboration d'un récit conduisent les autorités à envisager leur déploiement territorial dans plusieurs directions (le delta, la ville intérieure, le continent), l'incertitude dominant en même temps que renchérit l'hystérie constructive. Mais cette course infrastructurelle qui avait fait ses preuves pour 1997, peut-elle répondre à la question, alors que le futur sur lequel devraient s'appuyer ces projets n'est plus aussi optimiste?

\section{Conclusion : la ville-Narcisse}

Le métarécit infra-urbain qui s'achève ici provisoirement montre combien l'identification de la scène qui le porte et le réalise est essentielle pour comprendre les conditions de la naissance des projets, les méandres de leur existence, les rebondissements de leurs controverses, leurs succès et leurs impasses. L'efficace du projet ne se mesure pas seulement à la réalisation du discours par le construit même si, à Hong Kong, la planification réussit l'exploit de se réaliser (Haeringer, 2002). Il s'analyse aussi dans les interstices qui apparaissent entre les représentations et les 
pratiques, conduisant l'événement aéroportuaire à transformer la ville en même temps que celle-ci le façonne. C'est autour de cette notion de "ville-aéroport » que nous souhaiterions conclure.

Le " quantum lap » que réalise le déploiement de la " city of infrastructure » se fait selon un double mouvement, celui, centripète, que produit le déplacement de l'aéroport, balancé par celui, centrifuge, qui conduit le projet à être réapproprié par la scène urbaine hongkongaise. Pour mieux s'ancrer dans le réel de la ville, le récit conquérant et pionnier d'une extension des possibles s'alimente des débats, des oppositions, des rebondissements, la " ville-aéroport " se constituant comme une scène travaillée par l'instabilité des tensions entre attraction et disjonction qui secouent son territoire, des controverses entre partisans et opposants qui médiatisent son projet, des écarts entre l'image et l'usage qui modifient son expérience urbaine. Cette agrégation progressive se lit également dans la sémantique prolifique - "The new Airport in Central ", "City as airport versus airport as city ", "Bringing new airport back to the city " - dont les formules agissent comme des prophéties autoréalisatrices. La visibilité des pratiques aériennes en ville s'accompagne ainsi d'une invisibilité des distinctions que son infrastructure marque avec la ville, discours, concepts et politiques hybridant les deux termes.

Toujours grandir, ne jamais s'arrêter. Alors que l'horizon 2047 paraît incertain, les formes d'agrandissement que projette à nouveau Hong Kong montrent par l'éclatement des destins la fragilité du projet de " city of infrastructure ". Analyste des processus d'accélération qui façonnent notre monde contemporain, Hartmut Rosa évoque le terme de "résonance " pour qualifier la course acharnée dont sont l'objet nos sociétés et leurs villes (Rosa, 2014). Il reste à préciser quelles sont les limites de ce processus afin que la vibration ne conduise à la ruine. Dans son opus 2046 (sorti en 2004), le cinéaste hongkongais Wong Kar-Wai faisait un usage très particulier de la science-fiction. 2046 appartenait au passé et au futur, et si le film convoquait les signifiants communs du genre science-fictionnel, c'était pour invoquer une idée opposée à celle qui leur est traditionnellement associée. Ainsi le train supersonique se faisait-il vecteur d'immobilité, les allers-retours temporels menaient-ils à une impasse décisionnelle, et le concept de futur était-il dépendant de celui de mémoire. 


\section{Références}

Augé M. (1995), Non-lieux : introduction à une anthropologie de la surmodernité, Paris, Le Seuil.

Castells M. (1996), The Rise of the Network Society, Oxford, Blackwell.

Elliot D. (1998), " Hard times in Hong Kong a year after the handover: a bright new airport and a stalled economy », Newsweek, 13 juillet.

Farrell T. (1998), Kowloon Transport Super City, Hong Kong, Pace Publishing Limited, $112 \mathrm{p}$.

Fouchier V. (1993), "Hong Kong, comment gérer les fortes densités ? ", Perspectives chinoises, $\mathrm{n}^{\circ} 13-14$, p. 56-66.

Graham M. (1998), "Chek Lap Kok: Touchdown in Hong Kong », South Morning China Post, Asia Magazine, 15-17 mai, p. 9-12.

Gutierrez L., Portefaix V. (2000), Mapping HK, Hong Kong, Map Book Publishers.

Haeringer P. (éd.) (2002), La refondation mégapolitaine: une nouvelle phase de l'histoire urbaine?'Tome 1. L'Eurasie post-communiste. Moscou, Shanghai, Hong Kong, Paris, Centre de prospective et de veille scientifique, coll. "Techniques, territoires et sociétés ", n 36.

Hirsh M. (2016), Airport Urbanism: Infrastructure and mobility in Asia, Minneapolis (Minn.), Londres, University of Minnesota Press.

Hong Kong's Port and Airport Development Strategy: A Foundation for Growth, Hong Kong, Government Information Services, octobre 1991.

Ibelings H. (2002), Supermodernism, Architecture in the Age of Globalization, Rotterdam, Nai.

Jianfa S. (2010), "Assessing inter-city relations between Hong Kong and Shenzhen: the case of airport competition or cooperation ", Progress in Planning, ${ }^{\circ} 73$, p. 55-73.

Kam Ng M. (1993), « Strategic Planning in Hong Kong: Lessons from Territorial Development Strategy and Port and Airport Development Strategy ", The Town Planning Review, vol. 64, n 3, juillet, p. 287-311.

Kasarda J., Lindsay G (2011), Aerotropolis: How we'll live next, New York, Farrar, Straus and Giroux. 
Leung M. J. (1991), An analysis of the decision to build Hong Kong's second international airport, Thèse, Hong Kong University.

Li D. (1996), " Pearl City Here We Come, Forget the border and the crowds at Lowu. In 10 years' time the area stretching from Hong Kong to Guangzhou might well be the most dynamic piece of land in the world ", South China Morning Post, 6 juillet.

Loh C. (2001), "The once and future Victoria Harbour saved from the brink ", dans Hong Kong: defining the edge, Cambridge (Mass.), Harvard University, Graduate School of Design, p. 71-77.

Lorrain D. (éd.) (2011), Métropoles XXL en pays émergents, Paris, Presses de Sciences Po.

Matsuda N. (1997), "Hong Kong's Future as an "Airport City", Blueprint for the 21st century Hong Kong ", Space Design, n 394, p. 61-83.

McNeill D. (2014), "Airports and territorial restructuring, The case of Hong Kong ", Urban Studies, vol. 51, n 14, p. 2996-3010.

Nadal L. (1992), "L'Aeropolis du Kansai, utopie urbaine de la classe d'affaires japonaise ", Lumières de la ville, Formes Limites Échappées, n 6, novembre.

Orsay-Lam M.-H. (2002), "Un aéroport et une ville nouvelle. La transformation progressive de Hong Kong au gré des enjeux globaux et locaux ", dans Haeringer P. (éd.), La refondation mégapolitaine: une nouvelle phase de l'histoire urbaine? Tome 1. L'Eurasie post-communiste. Moscou, Shanghai, Hong Kong, Paris, Centre de prospective et de veille scientifique, coll. "Techniques, territoires et sociétés », n³6, p. 263-273.

Pryor E. G., Pau S.-H. (1993), "The Growth of the city, A historical review ", dans Lampugnani V. M., Hong Kong Architecture: The Aesthetics of Density, Munich, Prestel, p. 97-139.

Rocco Design Limited (2002), "Hong Kong Station and Development ", dans Hope E. (éd.), The City in architecture: Recent works of Rocco Design, Mulgrave (Vict.), Images Publishing Group, p. 120-137.

Rosa H. (2014), Accélération et aliénation : vers une théorie critique de la modernité tardive, Paris, La Découverte.

Roseau N. (2012), "Le Grand Paris des Infrastructures, un projet en crise ? ", dans Rouillard D. (éd.), L'Infraville : futurs des infrastructures, Paris, Archibooks, p. 53-64. 
Tiry C. (2003), « Hong Kong, un avenir urbain modelé par les transports ferroviaires ", Perspectives chinoises, $n^{\circ} 78$, juillet-août.

Tiry C. (2009), «Entre hypercentre et fragment d'aéroville, Hong Kong Station. Principes de mise en œuvre d'une mutation urbaine ", dans Prelorenzo C., Rouillard D. (éds), La métropole des infrastructures, $X X^{e}-X X I^{T}$ siècles, Paris, Picard, p. 153-166.

Thackara J. (2005), In the Bubble: Designing in a complex world, Cambridge (Mass.), The MIT Press. 


\title{
Les architectures événementielles, des infrastructures de la ville?
}

\author{
Sarra Kasri
}

La complexité de cerner et comprendre la ville est due aux diverses strates qui la forment. En effet, considérée comme territoire urbain saisissable de par ses logiques fonctionnelles, ses aspects morphologiques et ses représentations sociales, la ville peut être lue à travers ses dynamiques spatio-temporelles.

En s'attardant sur cette logique complexe de strates, depuis la révolution industrielle qui a propulsé l'expansion des diverses dessertes (électrique, d'assainissement, transports, télécommunication...), la lecture des territoires urbains peut se saisir suivant deux logiques : une première lecture en réseaux qui dépasse les morphologies tentaculaires propres aux infrastructures pour atteindre des logiques de connectivités qui les sous-tendent, comme l'explique Antoine Picon : "Pour parler de réseau, il faut de l'infrastructure physique, mais aussi des savoirs et des savoir-faire, des cultures professionnelles, en un mot des entités nettement moins matérielles, puisqu'elles résident pour partie dans la tête de ceux qui la possèdent et dans les relations qu'ils nouent entre eux» (Picon, 2014, p. 14).

Une deuxième lecture en référence aux grandes structures territoriales et urbaines (Picon, 2014) intéresse davantage cette contribution. Cette lecture est celle des repères urbains caractérisés par des échelles spatio-temporelles d'envergures leur conférant une visibilité et une événementialité ${ }^{1}$ urbaine incontestable. Ainsi, d'après Kevin Lynch (1969), la ville doit être lisible, par le biais de ses diverses représentations cognitives, elle doit être saisissable et appréhendable par les individus qui y vivent. Pour cela, il faut réussir à avoir une identification et une

1 Événement - événementielle : défini non pas au sens de l'éphémère mais plutôt au sens d'une ponctuation dans la ville : un repère. Au sens de K. Lynch (1969). 
configuration complète de ses lieux. L'identification d'un repère urbain consiste en sa reconnaissance, dans la mesure où identifier un élément suppose qu'on le distingue des autres choses, qu'on le reconnaisse comme une entité séparée. De ce fait, aspirer à être infrastructure revient à acquérir le statut de repère urbain à travers un marquage spatial d'envergure $^{2}$ dans la ville accentué par des potentialités de fonctions architecturales.

En effet, tout espace architectural renferme des potentialités de fonctions qui sont activées, questionnées et parfois détournées par les pratiques sociales. Ces potentialités peuvent être structurées suivant trois catégories : la première étant la fonction pragmatique qui tend à répondre à la finalité d'usage pour laquelle l'espace est créé, elle est en continuelle reconsidération durant sa période d'exploitation par la société ; la deuxième fonction est sociale, à la fois active et passive, l'architecture est façonnée par la société, mais peut contraindre à son tour les pratiques sociales qui définissent cette dernière : "À chaque société son espace, le spatial est une dimension du social " (Isnard, 1978) ; et enfin, la troisième fonction est imaginaire, elle résulte de la pluralité des représentations négociées et controversées. Cette potentialité est aussi importante que les deux précédentes puisqu'elle dépasse la qualification fonctionnelle pragmatique de l'espace pour le valoriser subtilement et ainsi lui permettre d'acquérir des instruments [repères sociaux], de créer l'événement et donc d'agir sur la société.

Ainsi, Manuel Castells énonce qu' " il y a un double mouvement d'organisation spatiale, l'espace des flux constitué par des réseaux connectant des lieux en une unité transterritoriale, et l'espace des lieux, enracinant le sens dans l'expérience vécue d'un territoire " (Castells, 2000) $)^{3}$.

2 Envergure : "Dans une œuvre comme le Centre de congrès de Lille comme dans ses écrits théoriques récents consacrés à la problématique du " gros projet ", un Rem Koolas se situe quant à lui aux frontières de l'architecture et des infrastructures, infrastructures là encore appréhendées de manière relativement traditionnelle, en référence aux grandes structures territoriales et urbaines " (Picon, 2014, p. 19).

3 L'ère des réseaux. Entretien avec Manuel Castells. Propos recueillis par Serge Lellouche. Publié le $1^{\text {er }}$ juin 2000 (version électronique) https://www.scienceshum aines.com/l-ere-des-reseaux-entretien-avec-manuel-castells_fr_12093.html(consulté le 21 juillet 2017). 
Decefait, questionnerles potentialités del'architectureévénementielle à aspirer à être infrastructure revient à l'interpeller sur ses capacités à faire sens. Ainsi, dans leurs quêtes de construction du sens, il semble intéressant de questionner ces projets repères urbains sur la nature de leurs identités-résistances ${ }^{4}$ qu'esquisse Manuel Castells. Dans quelle mesure ces architectures événementielles peuvent-elles être conçues comme des identités-projets ${ }^{5}$ porteurs de mutations organisationnelles et éventuellement de nouveaux ordres sociaux, politiques, économiques et idéologiques? Et ainsi, par extension, dans quelles mesures ces projets aspirent-ils à être infrastructure?

\section{L'imaginaire négocié des architectures événementielles}

Traversées à la fois par des controverses et des consensus, les architectures événementielles en questionnement perpétuel achèveront leurs itinéraires en devenant monuments faisant partie de la conscience collective d'une société, cependant, leur carrière sociale continue puisqu'elles seront toujours des sujets à débat, arbitrage, polémiques et surtout appropriations par plusieurs acteurs de la ville à des intervalles de temps différents. "Considérer un bâtiment seulement comme un objet statique, c'est comme observer inlassablement un oiseau volant haut dans le ciel sans jamais pouvoir saisir comment il se meut " (Latour et Yaneva, 2008, p. 3).

Ce qui nous invite à questionner la dynamique de ces architectures événementielles comme choses : ensemble de constituants controversés et négociés (Latour et Yaneva, 2008), échappant à la statique apparente

4 « Les identités-résistances construisent des projets identitaires communautaires, autour d'identités non généralisables, telles que la nation, le terroir, l'ethnicité et à certains égards la religion. Ce sont des identités fortes car elles s'appuient, de façon directe ou reconstruite, sur l'histoire, la géographie ou la mémoire collective. Mais ce sont des identités de résistance car elles ne peuvent pas reconstruire la société sur des valeurs partagées" (Castells, 2000).

5 " Les identités-projets sont des embryons de possibles formes nouvelles d'organisations sociales et d'institutions qui, en principe, peuvent modifier les rapports sociaux pour tous et construire une nouvelle hégémonie culturelle et politique " (Castells, 2000). 
de leurs esthétiques, à travers lesquelles tentent de cohabiter des imaginaires multiples, ceux des commanditaires, des concepteurs et aussi des usagers :

"Chacun sait qu'un bâtiment est un territoire contesté, et qu'il ne peut être réduit à ce qui est et à ce qu'il signifie, comme l'a toujours fait la théorie de l'architecture traditionnelle. Ce n'est qu'en tenant compte des mouvements d'un bâtiment, et en rendant soigneusement compte de ses "tribulations" que l'on pourrait exprimer son existence : ce serait l'équivalent de la liste complète des controverses et des réussites du bâtiment dans la durée, c'està-dire l'équivalent de ce qu'il fait, comment il résiste aux tentatives de transformation, autorise certaines actions des visiteurs tout en empêchant d'autres, énerve les observateurs, conteste les autorités municipales et mobilise diverses communautés d'acteurs" (Latour et Yaneva, 2008, p. 7).

Cette cohabitation fait sens dans la mesure où ces imaginaires ambitionnent tous à la construction d'une identité collective. Et dans la visée de mieux cerner les spécificités de ces architectures structurant à la fois l'espace public et l'identité sociale, une relation dialectique semble se dégager reliant trois notions : l'espace, le pouvoir et l'imaginaire. Dans ce sens, le symbole est défini en tant qu'une réalité matérielle (un bâtiment, une statue, une pièce de monnaie, etc.) qui communique quelque chose d'immatériel (une idée, une valeur, une identité...) affectant à des portions d'espace un nom, une identité et une raison d'exister. En d'autres termes, ces architectures événementielles semblent avoir un potentiel chargé de symbolique spatiale qui n'existe pas de fait, elle est produite par des codes sociaux qui se forment, se stratifient et se négocient dans l'histoire. Ce qui leur donne du sens, c'est la façon dont la société les interpelle et les instrumente.

Faisant partie du processus de la construction de l'identité collective, à l'image de cette dernière dans la mesure où elle " n'est autre que le résultat à la fois stable et provisoire, individuel et collectif, subjectif et objectif, biographique et structurel, des divers processus de socialisation qui, conjointement, construisent les individus et définissent les institutions " (Dubar, 1991, p. 113), ces architectures événementielles font l'objet d'une construction toujours inachevée. Et puisqu'elles s'inscrivent dans des dynamiques sociales, ces réponses spatiales ne font que refléter et exprimer ces dernières. D'après Castells, " l'espace devient l'expression de la structure sociale et son analyse revient à comprendre 
comment il est façonné par le système économique, le système politique et le système idéologique. L'articulation spécifique de ces trois systèmes s'appelle le système urbain " (Castells, 1972, p. 55).

Ainsi, les identités collectives ne forment pas à elles seules l'image d'une communauté, il y a aussi la présence de pouvoirs qui contribuent à l'identification de la société, soit comme une communauté de croyants, soit comme une nation de citoyens, soit comme un marché de consommation. En effet, on peut distinguer trois types de pouvoirs, un pouvoir religieux, un pouvoir politique et un pouvoir économique, ces divers pouvoirs cohabitent ensemble et participent, chacun à sa manière, au façonnement de l'identité collective ainsi qu'à ses représentations. Et c'est à travers l'architecture événementielle que le pouvoir à la fois met en valeur sa présence dans le paysage sociétal, mais aussi façonne à son tour ce dernier en usant de procédés démocratiques pour légitimer son empreinte à l'instar de la procédure des concours. L'architecture événementielle consiste alors à peupler l'espace de la ville de signifiants qui sont autant de médiations esthétiques du fait politico-social. Les projets ont ainsi une double signification, puisquà la fois ils font de l'espace urbain un espace esthétique, un paysage, et ils y inscrivent les signifiants et les traces de l'histoire politique de l'urbanité.

De ce fait, les lieux sont mis en scène à travers l'événement architectural avec pour but de signifier à la fois le temps, l'histoire avec ses conjonctures et enfin sa mise en mémoire, produisant ainsi une série d'identités urbaines. De la sorte, ce processus de création des « identités urbaines » est basé sur l'utilisation d'un certain nombre de signes, objets spatiaux et architectures événementielles qui renvoient à des imaginaires et représentations de la ville, s'appuyant sur des représentations sociales négociées. Pour illustrer ce qui précède, cette contribution se réfere à une sélection de projets présidentiels français et tunisien qui ont eu recours à la procédure de concours pour leurs processus de réalisation.

\section{Projet présidentiel : une architecture événementielle}

" Je voudrais passionnément que Paris possède un centre culturel comme on a cherché à en créer aux États-Unis [...], qui soit à la fois un musée et un centre de création, où les arts plastiques voisineraient avec la musique, le 
cinéma, les livres, la recherche audiovisuelle, etc. Le musée ne peut être que d'art moderne, puisque nous avons le Louvre. La création, évidemment, serait moderne et évoluerait sans cesse. La bibliothèque attirerait des milliers de lecteurs qui du même coup seraient mis en contact avec les arts. Tout cela coûte cher $[\ldots]$, mais si l'objectif est atteint, ce sera une réussite sans précédent " (Le président Georges Pompidou, Le Monde, 17 octobre 1972).

«Dans le but de consolider l'infrastructure du secteur culturel et de le renforcer au moyen d'espaces modernes qui soient adaptés à l'évolution de ses activités et à la diversification de ses caractéristiques, nous avons décidé d'entamer, à partir du début de l'année 2001, la construction à Tunis d'une Cité de la culture. [...] À l'heure où le monde assiste à des progrès spectaculaires dans le domaine des sciences et du savoir, nous nous devons d'œuvrer à la propagation, au sein de notre peuple, de la culture scientifique et à son initiation aux nouvelles technologies de la communication et de l'information, de lui en garantir les sources d'accès et l'utilisation judicieuse, et de l'aider à être mieux au fait des apports de son époque " (Le président Zine el-Abidine Ben Ali, Discours à l'occasion du $13^{\mathrm{e}}$ anniversaire du Changement, 7 novembre 2000).

S’inscrivant dans une tradition française des présidents bâtisseurs, Georges Pompidou dans un élan de modernisation de Paris a décidé de doter cette ville d'un centre national d'art et de culture. "Cette "ouverture personnelle" que constitua le projet de Beaubourg pour Georges Pompidou apparaît comme le révélateur de volontés technocratiques et politiques nouvelles " (Aymone, 2007). Suite à une procédure de concours international organisé en 1971, dans laquelle 681 groupements d'architectes ont concouru, le projet sélectionné est celui de Richard Rogers et Renzo Piano. Cette architecture qui s'inscrit dans une mouvance valorisant l'esthétique de la technique a suscité une grande polémique dans la mesure où elle a bousculé tous les codes architecturaux de son contexte urbain parisien. Par la suite, la tradition de la réalisation d'imposants projets d'architecture au service de la culture et du pouvoir politique s'est bien installée dans la V République. Ainsi, chaque président a ambitionné de laisser une empreinte urbaine de son passage à la tête de l'État français. De Valéry Giscard d'Estaing avec le musée d'Orsay à Jacques Chirac avec le musée du Quai Branly en passant bien évidemment par François Mitterrand avec la Pyramide du Louvre, l'Institut du monde arabe et la Bibliothèque nationale de 
France, tous ces présidents ont contribué, chacun à sa manière, au façonnement urbain de la ville de Paris, de son esthétique, mais aussi de son vécu.

Par ailleurs, il est à souligner que cette tradition politique des "présidents bâtisseurs » s'étend au-delà des frontières françaises. En effet, du Brésil avec l'aménagement de Brasilia par Niemeyer à l'Inde avec l'aménagement de Chandigarh par Le Corbusier et plus récemment sur une échelle urbaine plus restreinte en Tunisie, les projets présidentiels de la Mosquée de Carthage et celui du projet de la Cité de la Culture, cette mouvance inspire les gouverneurs et façonne les pays. Tradition qui prend ses racines des "Faits du Prince », héritée de la Royauté et reprise ultérieurement à la fois par des régimes démocratiques et des régimes autoritaires pour mettre en scène et sublimer l'étendue du pouvoir.

Cependant, l'analyse de ces projets architecturaux ne doit pas se cantonner à la compréhension de la traduction des desseins du politique en des dessins de l'architecte. Puisque la lecture des configurations de cette architecture monumentale consiste à saisir et à comprendre non pas l'histoire, mais davantage les histoires ainsi que l'évolution et la dynamique des changements et des ponctuations idéologiques qu’a connus la société concernée. Ainsi, le récit de cette architecture certes débute au moment où le politique l'aspire, mais il se poursuit à travers les diverses phases de négociations économiques, techniques, mais aussi sociales, culturelles et architecturales. Devenir architecture événementielle revient en quelque sorte à dépasser la condition première des bâtiments à être support de projection du social pour devenir en plus un acteur social actif de la ville ayant la capacité d'associer de multiples acteurs humains et des acteurs non humains en des périodes de temps différentes. En effet, à travers les différentes controverses que de pareilles architectures peuvent créer, plusieurs groupes de personnes sont enrôlés. Groupes de soutien ou collectifs anti-projet produisent et instrumentalisent de multiples manifestations dérivantes du projet architectural sous forme de manifestes, écrits journalistiques, estimations budgétaires et de diverses expertises mobilisant des réglementations urbaines et des écrits sur l'esthétique et l'identité. De ce fait, source de vifs questionnements et jugements, ces architectures monumentales créent l'événement ou l'événementialité dans l'urbain puisqu'elles 
ne laissent pas indifférent. "[Elles] agissent, résistent, ordonnent, mobilisent et rassemblent différentes communautés d'acteurs [...]" (Yaneva, 2009, p. 199) ${ }^{6}$.

À travers ces projets présidentiels, les commanditaires (présidents de la République) ambitionnent ainsi de s'inscrire dans l'espace et de marquer par leurs empreintes le temps. Leurs quêtes dépassent celle de la construction de simples bâtiments au service de la ville. L'imaginaire qui les anime est un imaginaire magnifié : celui de s'associer à l'histoire du pays et à la grandeur de ce dernier ${ }^{7}$. Certains diront même que, par leurs desseins, ces commanditaires de projets présidentiels défient la mort en se projetant à travers des monuments nationaux. Et pourtant, en dépit de la grandeur de leurs convoitises, ces commanditaires s'inscrivent dans un contexte où le fait du prince se heurte aux exigences de la démocratie, "cette forme de résurgence monarchique au cœur de l'État républicain " (Lengereau, 2001, p. 214). Comment magnifier alors leurs empreintes avec les instruments de leurs sociétés et loin des inconvénients d'une certaine monarchie ou dictature ? Pour répondre à cette problématique, le système des concours défini comme la mise en concurrence des concepteurs semble être une pertinente réponse à ce dilemme puisqu'il concrétise, dans une certaine mesure, un glissement du Fait du Prince au Fait du Président ${ }^{8}$.

6 " A building exists if it acts, resists, affords, compels, challenges, mobilizes, bugs, and gathers different communities of actors [...]”(Yaneva, 2009, p. 199).

7 Georges Pompidou, en énonçant sa vision de la durée de vie du Centre Beaubourg à " cinq siècles ", reflète l'imaginaire de la pérennité de son empreinte, de son imaginaire d'une France en mouvement, d'une modernité. Cité par Renzo Piano.

8 Commentant ses différents projets présidentiels, F. Mitterrand déclarait : «Il y a des règles très strictes, jurys nationaux ou jurys internationaux. [...] Des règles internationales président à la constitution et au fonctionnement des jurys. On ne voit pas très bien un homme soudain se substituer à eux cent pour cent. On peut dire non, je préférais celui-là... discuter... Ce sont des gens très obligeants mais c'est difficile de faire plus " (F. Mitterrand, interview accordée à B. Pivot sur France 2 le 14 avril 1995). Cependant, en dépit de l'apparente suprématie du choix des jurys de concours, le cas de l'Opéra Bastille reste un fait marquant de la réception d'un projet lauréat qui ne correspond pas à l'imaginaire du commanditaire ni à celui des membres de jury, puisqu'il y a eu confusion sur l'identité de l'architecte à travers la similitude des styles architecturaux du projet lauréat de Carlos Ott avec l'empreinte de Richard Meier. 
Ainsi, Faits du Prince par excellence, mais inscrits dans un contexte démocratique, le Centre Beaubourg et la Pyramide du Louvre sont d'excellents exemples d'architectures événementielles, considérées comme des acteurs sociaux actifs, issues du système des concours internationaux. Tracer les ponctuations de leurs existences serait égal à la liste étendue des performances architecturales et des controverses auxquelles ils semblent se prêter au fil du temps.

\section{Le système des concours : processus de fabrication d'architectures événementielles}

Comment produire une architecture monumentale et événementielle, censée marquer l'histoire en démocratie ? Dans ce cadre, le concours constitue un passage "intermédiaire ", permettant de pérenniser la démarche (ou la forme) tout en tentant de préserver la déontologie démocratique.

Cité dans la préface de l'ouvrage L'apogée des concours d'architecture d'Aymone Nicolas (2007), Diderot constate :

"Il est une connaissance entièrement négligée par ceux qui sont à la tête de l'administration : c'est celle de l'architecture. Cependant ce sont eux qui ordonnent les monuments publics, qui font le choix des artistes, à qui l'on présente les plans, et qui décident de ce qu'il convient d'exécuter. Comment s'acquitteront-ils de cette partie de leurs fonctions qui touche de si près à l'honneur de la nation, dans le moment et dans l'avenir, s'ils sont sans principes, sans lumières et sans goût? Il n'y a point de sottises qui durent plus longtemps et qui se remarquent davantage que celles qui se font en pierre et en marbre. Un mauvais ouvrage de littérature passe et s'oublie ; mais un moment ridicule subsiste pendant des siècles, avec la date du règne sous lequel il a été construit. Il faut avoir la vue bien courte ou bien longue pour négliger cette considération. »

En réponse à ces propos, Hubert Védrine exprime sa conviction que la meilleure façon de construire des monuments publics échappant à toute influence et contrainte architecturale, économique ou bureaucratique reste le système des concours. Dans la continuité de ces réflexions, cette partie tente donc maintenant de questionner l'apport de la procédure 
concurrentielle dans la fabrique des architectures événementielles définies en tant que projets présidentiels et considérées comme des infrastructures de la ville.

Les concours, inscrits dans leurs contextualités, constituent une approche particulière de la fabrication des monuments de la ville, et ce par la mise en concurrence de concepteurs suivant un processus normé et rationnel visant à éviter l'arbitraire et à atteindre le consensus, " le passage d'un mode de création individuel à un mode de création beaucoup plus collectif " (Aymone, 2007, p. 182, citant le rapport du jury du concours de Beaubourg). Ainsi, il y a transfert des logiques de la commande des mains du commanditaire vers l'arène publique, mettant l'accent davantage sur les performances architecturales et de créativité'.

Basées sur les nouveaux concepts issus de la révolution industrielle et de la Révolution française (fonctionnalisme, rationalisme et pluralisme), les prémisses de la procédure des concours ont vu le jour, se définissant comme une mise en compétition démocratique des architectes reconnus par l'État dans le but d'apporter une réponse qui se veut la plus optimale pour le projet en question.

$\mathrm{Au}$ niveau international, la genèse du règlement des concours internationaux a vu le jour en 1911 à Rome lors du neuvième congrès international d'architectes, à travers une série de réunions

Au cours de la période des Lumières, la réalisation des bâtiments publics était limitée au cercle très restreint des architectes du roi (issu d'une formation académique d'élite), la notion de concours n'existant pas vraiment, du moins pas à l'image des concours techniques lancés à la Renaissance en Italie (Brunelleschi...). Cependant, il était courant que l'on demandât plusieurs dessins à différents architectes pour tel ou tel projet, et ce successivement ou simultanément. Avec l'avènement de la Révolution française, les bâtiments publics et les académies d'architecture ainsi que les architectes du roi étaient visés par l'oppression autant que le pouvoir renversé. En reprenant plus ou moins la même logique de l'académie et des architectes du roi, une autre institution a vu le jour, l'école des beaux-arts et, avec elle, le conseil des bâtiments civils, formé des lauréats du prix de Rome, dont le rôle consiste en la réalisation des monuments de l'État avec des actions se répandant sur tout le territoire et aboutissant à un conformisme esthétique de tout le paysage architectural de la France. Devenant de plus en plus accaparant, le conformisme de l'école des beaux-arts et du conseil des bâtiments civils engendra une polémique qui divisa les architectes mais aussi les maîtres d'ouvrage de cette époque, et ce entre un héritage académique et une réforme profonde. Cette dernière a pu s'accomplir dans les sillages de Mai 68. 
visant à définir et à réglementer les concours internationaux. Les travaux de ce congrès ont permis la fixation d'un certain nombre de recommandations esquissant les principes directeurs des concours internationaux (procédures de préparation des concours, formation des jurys, suite donnée au jugement...) : "Les règles formulées en 1971 (pour les concours internationaux) : un programme précis, mais pas contraignant, une attention des jurés à l'innovation et à la créativité, l'anonymat comme garantie de l'égalité républicaine, et l'attribution du dernier mot au président de la République " (Aymone, 2007, p. 181). Plusieurs événements internationaux ont ainsi suivi, notamment :

- La Haye en 1927, lors du XI ${ }^{\mathrm{e}}$ congrès international des architectes : élaboration d'un modèle type de réglementation ;

- Winchester en 1928 et Strasbourg en 1929 : l'union francobritannique des architectes demande la collaboration et l'appui de la Société des Nations ; maintes recommandations ont découlé de cette collaboration améliorant la procédure des concours internationaux surtout au niveau de la formation des jurys et de la préparation des programmes des projets ;

- 1948 : création de l'Union internationale des architectes et reprise des travaux sur la réglementation des concours internationaux ;

- Venise 1952 : les travaux de la conférence générale des artistes, ainsi que ceux de l'Union internationale des architectes (UIA) et de l'Organisation des Nations unies pour l'éducation, la science et la culture (UNESCO), aboutissent à la nécessité de fixer une convention entre les gouvernements, avec l'adoption d'un règlement qui définit l'ensemble des paramètres relatifs à la procédure des concours internationaux d'architecture et d'urbanisme ;

- 1956 : Conférence générale de l'UNESCO et adoption du règlement type concernant les concours d'architecture et d'urbanisme; il est demandé à l'Union nationale des architectes de veiller à son application. Ce règlement a été révisé en 1978.

Ainsi, les concours, inscrits dans leurs contextualités, consistent en une mise en concurrence démocratique et transparente d'un groupement de concepteurs reconnus. Cette démarche organisationnelle constitue une approche particulière dont l'objectif vise la production d'architectures 
hautement représentatives et négociées de l'ensemble des imaginaires qui mettent en valeur et construisent l'identité d'une époque, d'un groupement social ou d'une mouvance donnée.

Cependant, il semble pertinent de questionner cette " utopie des concours» (Quincerot et Nicolas, 1980) au regard à la fois du pragmatisme de leurs méthodes, de leurs inscriptions dans les controverses qui les précèdent, mais aussi qui les suivent, et enfin au regard de l'empilement ou de l'enchevêtrement des imaginaires (Picon, 2014) des multiples acteurs enrôlés. En effet, des conseillers des présidents aux rédacteurs de l'énoncé du concours en passant par les différentes instances du concours, les concurrents et enfin les membres de jury, tout ce processus s'ancre dans une chaîne de traduction d'imaginaires et de représentations de ce que c'est que la société, à quoi elle aspire et de l'empreinte que souhaite laisser le Président.

Quand François Mitterrand, Georges Pompidou ou Zine el-Abidine Ben Ali choisissent de passer par la procédure des concours pour la réalisation de leurs projets présidentiels, ils étaient tous en quête du meilleur projet architectural qui exalte leur imaginaire. Cependant, la démarche concurrentielle dépasse cette simple sélection naturelle des projets pour légitimer ces architectures événementielles à travers l'établissement d'un consensus " rationnel " issu essentiellement du préjugé d'innocence (Quincerot et Nicolas, 1980) des concours. En effet, ce capital de légitimité est acquis à travers une structure éphémère jouant le rôle de relais par la mise en forme d'une commande ou d'un imaginaire politique, sa mise en débat par un certain nombre de concurrents et de membres de jury et enfin sa restitution à la société sous forme d'un projet architectural qui aspire à devenir un événement.

Ainsi, le système des concours " ne prend du pouvoir que pour le redistribuer aussitôt à d'autres, avant de prononcer sa propre disparition. L'autorité du concours, c'est de se présenter comme le contraire d'une institution : précisément, comme la procédure d'exception apte à prendre le relais des institutions permanentes qui, en certaines occasions, seraient suffisantes " (Quincerot et Nicolas, 1980, p. 91).

Cependant, en dépit de toutes les dispositions de distanciation que permettent les concours, les projets dont ils sont issus font une carrière de monuments contestés durant la phase de leurs exécutions, comme ce fut le cas à la fois pour la Pyramide du Louvre, qui nécessita sa simulation 
en vraie grandeur (avec des cordes) avant sa réelle construction, mais aussi pour le Centre Beaubourg ${ }^{10}$, qui faillit avorter sans l'action et le soutien de Jacques Chirac (Premier ministre à l'époque), et enfin pour la Cité de la Culture en Tunisie dont le chantier encore en cours a été ponctué de multiples arrêts et ce depuis plus d'une quinzaine d'années.

En conclusion, comme on l'a montré précédemment, le propre des projets présidentiels est qu'ils sont intrinsèquement porteurs d'un pouvoir légitimé par un processus qui se veut rationnel et objectif. En ce sens, les projets présidentiels, par la complicité du pouvoir et du système des concours, œuvrent à être "infrastructure monument " dans la mesure où ils intègrent la conscience collective de la société et des lieux couplés à une spatialité d'envergure remarquable. Cependant, au-delà de la validation de leur potentiel à devenir infrastructures, dans leurs quêtes de construction du sens, il semble intéressant de questionner ces projets présidentiels sur la nature de leurs identitésprojets (Castells, 2000), porteurs de mutations organisationnelles et éventuellement de nouveaux ordres sociaux, politiques, économiques et idéologiques. En effet, partant de semblables commandes, les projets du Centre Beaubourg et de la Cité de la Culture semblent avoir des identités-projets distinctes. Au-delà d'une esthétique architecturale ou d'une réponse programmatique, ce qui les différencie s'avère être plus de l'ordre des potentialités de restructuration des rapports sociaux et des itinéraires d'évolution de la société. Et c'est dans ce sens que semble se cristalliser davantage l'identité d'infrastructure pour les architectures événementielles.

\section{Références}

Castells M. (1972), La question urbaine, Paris, F. Maspero.

Castells M. (2000), « L'ère des réseaux. Entretien avec Manuel Castells. Propos recueillis par Serge Lellouche ", L'Ére des réseaux, hors série, $n^{\circ} 29$, $1^{\text {er }}$ juin.

Dubar C. (1991), La socialisation. Construction des identités sociales et professionnelles, Paris, Armand Colin.

10 Le récit détaillé du concours international pour la réalisation d'un Centre d'art est développé dans Lengereau (2001, p. 205-220). 
Isnard H. (1978), L'espace géographique, Paris, Presses universitaires de France.

Latour B., Yaneva A. (2008), "Donnez-moi un fusil et je ferai bouger tous les bâtiments. Point de vue d'une fourmi sur l'architecture ", dans Geiser R., Explorations in architecture: Teaching, Design, Research, Basel, Birkhäuser, p. 80-89.

Lengereau E. (2001), L'État et l'architecture 1958-1981 : une politique publique?, Paris, Picard.

Lynch K. (1969), L'image de la cité, Paris, Dunod.

Nicolas A. (2007), L'apogée des concours d'architecture : l'action de l'UIA 1948-1975, Paris, Picard.

Picon A. (2014), La ville des réseaux, un imaginaire politique, Paris, Manucius.

Quincerot R., Nicolas M. (1980), Concours d'architecture 1920-1940. Une qualité en projet, Genève, C.R.A.A.L, Université de Genève.

Yaneva A. (2009), The making of a building: A pragmatist approch to architecture, Oxford, Peter Lang.

"Ma grandeur de la France ", émission réalisée par B. Delais, http://www. ina.fr/video/CPD12000394 (consulté le 21 juillet 2017).

"L'ère des réseaux. Entretien avec Manuel Castells. Propos recueillis par Serge Lellouche ", publié le $1^{\text {er }}$ juin 2000, https://www.scienceshumaines. com/l-ere-des-reseaux-entretien-avec-manuel-castells_fr_12093.html (consulté le 21 juillet 2017). 


\title{
Naissance d'une infrastructure
}

\author{
Elsa Vivant
}

Décrire une infrastructure, ceux qui la conçoivent, ceux qui la produisent, ce(ux) qu'elle relie, ce(ux) qu'elle transforme, ce(ux) qu'elle révèle. Tel est le propos du livre de Maylis de Kerangal, Naissance d'un pont, dans lequel l'auteur nous raconte l'histoire de la construction d'un pont à Coca, petite ville ordinaire de Californie, à travers la présentation de son contexte de production, des intentions de son maître d'ouvrage, des contraintes qui se posent à la réalisation et de ceux qui vont rendre possible cette production, les ingénieurs et ouvriers, présents ou non sur le chantier. Reprenant les intentions de cet ouvrage collectif, comment la lecture de ce roman peut-elle contribuer à une réflexion sur les infrastructures, tant dans leur matérialité qu'en tant qu'analyseur du monde social ?

En quoi la fiction, comme art mimétique (Schaeffer, 1999), peutelle, par une forme romanesque, rendre compte de la complexité des processus de production de l'espace ? Plusieurs auteurs interrogent le rôle cognitif et anthropologique de la fiction dans son rapport au réel (Vermandel, 2013 ; Schaeffer, 1999 ; Becker, 2009 ; Chérel et Pasquier, 2013). Pour Schaeffer (1999), la fiction, entendue comme une feintise ludique partagée (du jeu d'enfant à la réalité virtuelle en passant par le roman) est une forme parmi d'autres d'interaction avec la réalité et de sa représentation. Comme l'ensemble des arts mimétiques, la fiction ne cherche pas à induire son récepteur dans l'erreur (prendre la fiction pour le réel), mais, à travers des techniques fictionnelles (ce qu'il appelle les leurres préattentionnels), à le faire entrer dans la fiction, dans un monde possible qui contribue à produire le réel ou des connaissances sur ce réel. La fiction peut ainsi être considérée comme un mode de représentation de la société, à condition de s'accorder sur une convention partagée : ceci est une fiction. Certains auteurs questionnent les modes d'écriture de la recherche, la mise en forme des faits s'apparentant à leur mise en récit, 
voire en fiction. L'écriture fictionnelle serait, pour eux, une manière de représenter le réel et de produire des connaissances. Renvoyant aux travaux de Vaihinger, Vermandel rappelle le caractère heuristique de la fiction dans le processus de production de connaissances en favorisant la jonction entre imagination et connaissance. Les fictions jouent ainsi un rôle dans l'activité spéculative de la pensée scientifique, par exemple par la formulation d'hypothèses. L'écriture scientifique serait un processus de production des faits et de représentation de ces faits. "Connaître, ce n'est pas simplement saisir ou élucider le monde (et le réel) par un acte d'objectivation, dont le langage scientifique serait l'outil, le calque ou l'empreinte, c'est aussi reconfigurer le monde, le transfigurer par l'écriture afin que le sens advienne. C'est notamment de ce point de vue que le rôle de la fiction peut s'éclairer et trouver sa fécondité dans la pensée scientifique »(Vermandel, 2013). En référence à Latour, Becker rappelle que la production des représentations consiste en une série de transformations, comme le font les scientifiques en transformant plusieurs fois leurs matériaux avant d'aboutir à une représentation des résultats acceptable par tous (Becker, 2009). De ce fait, toute représentation n'est que partielle et ne rend compte que de ce que l'émetteur sait de cette réalité. Mais tout artéfact ne devient représentation du réel qu'à partir du moment où un récepteur effectue un travail d'élaboration et d'interprétation pour lui donner un sens selon sa propre subjectivité (Becker, 2009). Un romancier peut, dans son œuvre littéraire, produire une analyse sociale. Il s'adresse à une communauté interprétative (le monde littéraire), mais peut en toucher d'autres, comme le chercheur en infrastructure qui voit, par la fiction, son imagination sociologique et méthodologique stimulée. D’ailleurs, la démarche d'écriture de Maylis de Kerangal rappelle certaines propositions de Bruno Latour concernant les modes de description et de représentation de la société (Latour, 2006). L'auteure s'attache en effet à décrire et à déployer tous ceux que ce pont fait agir, tous ceux (humains et non humains : ouvriers, oiseaux, eaux tumultueuses du fleuve...) qui sont concernés par la construction de ce pont. Elle mobilise différents moyens littéraires (l'énumération, l'usage de termes techniques, les figures de héros) pour rendre compte de la complexité de ce réseau d'actants et de la technicité de la production de cette infrastructure. Elle explique avoir travaillé de manière très documentée, sur des ponts mythiques (Millau, Brooklyn, Tancarville, Golden Gate) et s'être 
inspirée du réel pour inventer les lieux, l'histoire, les personnages. On pourrait qualifier ce roman de fiction documentaire, qui reconfigure le réel à partir de plusieurs situations très concrètes décrites dans leur matérialité et leur technicité et qui trouble le rapport au réel par l'usage de la fiction. Dans des entretiens réalisés avec des critiques littéraires, l'auteure dit se fixer à elle-même l'enjeu de représenter la construction du pont comme la plus plausible possible, la plus proche du réel. Sa gageure serait qu'un ingénieur des ponts et chaussées ferme le livre en étant convaincu que le pont est solide, que "ça tient ". "Ça tient ", expression à double sens pour questionner l'expérience du lecteur : à la fois une histoire qui se tient, qui embarque le lecteur dans un univers de fiction créé par l'auteur, et un pont qui tient en tant que pont. En cela, de par sa propre revendication d'ancrer sa fiction dans le réel, on peut interroger en quoi cette fiction représente le réel et ce qu'elle en représente.

Toute description ou toute explication d'une œuvre est orientée par le point de vue que l'on prend pour le décrire. Michael Baxandall (1991) a utilisé le cas de la construction d'un pont sur le Forth, en Écosse, pour mettre en évidence comment toute description d'un objet intentionnellement créé par l'homme traduit une interprétation sur les intentions de son auteur. Il raconte l'histoire de la création du pont sur le Forth en identifiant vingt-quatre moments clés ou causes expliquant la décision de construire ce pont, et les choix qui ont conduit à construire ce pont-là, tel qu'il est. Cela va des contraintes de l'environnement (la vase dans le lit de la rivière, le vent), celles imposées par d'autres acteurs (le tirant d'air pour les bateaux de l'armée, la concurrence entre les compagnies de chemins de fer), d'événements extérieurs (l'accident d'un autre pont qui délégitime son constructeur), l'histoire personnelle du concepteur (famille de sidérurgistes, intérêt pour l'histoire de la construction), l'évolution des technologies à l'époque (l'acier). Pour M. Baxandall, la manière d'articuler ces différents éléments explicatifs produit un sens particulier. D'autres articulations des mêmes éléments produiraient d'autres récits. Celui qui écrit et décrit le pont fait des choix selon ce qui lui apparaît important pour comprendre ce qu'il veut comprendre. La description d'un pont reflète la subjectivité de celui qui décrit. Puisque, si on suit Baxandal, l'explication d'un objet (ici le pont tel qu'il est décrit dans Naissance d'un pont) dépend du point de vue 
que l'on prend pour le décrire, il s'agit ici d'alimenter la réflexion sur les infrastructures en identifiant dans le roman ce qui sous-tend la réalisation de l'infrastructure, ce qu'elle implique, ce qu'elle transforme, ce qu'elle révèle. L'infrastructure et sa construction apparaissent ici comme un moyen d'analyser les transformations territoriales (de la colonisation de l'Amérique à l'urbanisation contemporaine), l'articulation des différentes échelles de l'action collective, les modes d'organisation mondialisée du travail par projet. Ces différentes perspectives se rejoignent à travers la dialectique territorialisation/déterritorialisation de l'infrastructure, dans son rapport à l'espace (politique, géographique et social) auquel elle se heurte et qu'elle transforme. L'espace social de l'infrastructure est celui du chantier de construction du pont et de ses hiérarchies. L'espace géographique est bousculé par l'arrivée du pont et le désenclavement qu'il promet (des quartiers de l'autre rive et de la forêt). L'espace politique est celui des conflits et luttes d'influence et d'intérêts à l'œuvre autour de l'ouvrage dans un jeu d'acteurs multiscalaire. La construction d'un pont est à la fois ce qui désenclave un territoire et ce qui agrège des individus, venus du monde entier, sur un périmètre et pour un temps donnés.

\section{Territorialisation}

Le pont est avant tout un ouvrage d'art qui relie deux rives et deux mondes : la forêt et la ville mondialisée. Il s'inscrit dans un paysage, le désenclave, introduit le flux et le mouvement, et par là même le transforme durablement et le projette dans la globalisation. La territorialisation de l'infrastructure apparaît par son ancrage dans l'histoire de la ville, dans un paysage, à travers le jeu d'acteurs local (le maire, les propriétaires de ferry, les riverains, les autochtones), le quotidien du chantier et des travailleurs. La description du chantier fait de l'infrastructure, par sa matérialité, l'objet de l'action du roman et le produit de l'action organisée d'acteurs institutionnels, mobilisant le travail d'individus au destin singulier.

L'histoire de Coca est celle des espaces qui se désenclavent, qui s'ouvrent au monde et mutent de manière irréversible. Inventer le lieu où se déroule le roman (sa géographie, son histoire, ses paysages, ses habitants) permet de fonder les accroches fictionnelles et une 
convention de lecture, tout en mobilisant des imaginaires partagés. Ville fictionnelle, Coca a des airs de dernier Far West. La Californie des westerns hollywoodiens, du mythe américain, les grands espaces, le paysage de la frontière. Ce pourrait être aussi dans le nouveau Far North West que constituent les terres riches en minerais du Yukon ou en sables bitumeux de l'Alberta où affluent les travailleurs en recherche d'argent rapide sans regarder à la besogne. Des agglomérations où alternent motels et fast-foods, devant lesquels s'alignent les pick-up crasseux. Coca, son maire, son pont, ses ambitieux, ses promesses d'un avenir flamboyant, réécrit son futur, d'un passé pourtant ordinaire dans ce grand ouest des espoirs, de la colonisation des terres, des plaines et des hommes. Missionnaires, pionniers, émigrés européens, chercheurs d'or, aventuriers ont construit, au bord d'une rivière, cette ville en peu de temps, par quelques murs de bois au milieu de la boue, malgré les maladies, les bêtes sauvages et contre les autochtones résistant à l'accaparement de leurs terres. Un vieux pont construit au début du siècle avait déjà permis la colonisation de l'autre rive, quartiers autoconstruits où habitent les plus miséreux de la ville. Ces bas-fonds étaient jusqu'alors restés en marge du développement urbain de l'après-guerre (motels, fast-food, centres commerciaux, lotissements pavillonnaires, zones industrielles) sous la houlette spéculative des vieilles familles qui s'étaient réservé les meilleures terres dès la fondation de la ville. Le projet de nouveau pont marque une rupture dans le récit de Coca. Désormais la bourgade du Far West se veut métropole. Rien n'ébranle la volonté de son nouveau maire et ses rêves de grandeur.

La description des gestes techniques et des contraintes matérielles de la construction du pont opère comme un procédé romanesque qui donne à voir ce qu'implique la production d'un objet sociotechnique en termes de compétences, de matériaux, de décisions, de métiers et d'expertise. Tout commence par un concours, pour communiquer sur la ville davantage que pour identifier la meilleure solution technique, remportée par l'architecte le plus séducteur, par ses mots, ses gestes, sa maîtrise de soi, sa prestation publique, son discours sur le paysage. Le prestige du pont (et de la ville) se fonde sur les ambitions qui s'y croisent, entreprises et architectes alléchés par un juteux contrat. Ensuite viennent les considérations matérielles et techniques, le concours de maîtrise d'œuvre, le choix du consortium de construction sur la foi de calculs, 
d'évaluations, de projections, de relevés, de ristournes et peut-être aussi d'enveloppes plus ou moins épaisses. Le symbolique et les beaux discours laissent la place à la technique, au pragmatisme et à la matérialité. À l'écart de la ville, dissimulé par des palissades, commence le chantier, village temporaire, fait de préfabriqué, d'installations techniques, de vestiaires, de routes, et des centaines d'hommes - et quelques femmes qui chaque jour arrivent, se changent, déjeunent, déconnent, travaillent, et qu'il faut encourager, réprimander, former, dans le froid, la nuit, la pluie, le vent, le bruit, la boue, la neige, la hauteur. Un chantier de cette envergure, ce sont des décisions à prendre, des problèmes techniques à résoudre, des missions impossibles à surmonter, des tâches à planifier. L'avancée des travaux se compte en millions de mètres cubes de boue dégagée, de béton coulé, de câbles d'acier déroulés. Seule territorialité de l'infrastructure : les réalités géomorphologique et hydrologique du site qui impliquent des choix techniques spécifiques. Depuis la ville, rien ne laisse soupçonner les excavations, les dragages, les explosions de dynamite, rien si ce n'est l'afflux des ouvriers dans les bars qui viennent convertir leur paye en plaisirs passagers.

À travers la construction du récit, interviennent différents acteurs, différents métiers, différents personnages. Sans eux, rien n’advient, ni pont ni récit. Ils sont là pour accomplir les tâches nécessaires à la construction du pont, et par là même font avancer l'action du récit. Construire un pont, c'est une épopée collective où se façonnent des destins individuels. L'espace social du chantier est traversé par quelques figures héroïques qui révèlent les rapports sociaux, mais aussi ce que pour chacun ce pont implique, ce qu'il change dans leur vie, les espoirs qu'il fait naître. Le monde de la construction n'est pas un espace social homogène. Il a ses hiérarchies d'autorité et de contrôle sur le travail effectué. Il est aussi parcouru par d'autres hiérarchies, celles du prestige de certains métiers, comme les plongeurs qui arrivent un matin, tels des superhéros, pour vérifier les piles de ponts construites dans la rivière. À l'inverse, des tâcherons interchangeables, licenciables au moindre prétexte, ont pour seule arme dans les rapports de force leur nombre et leur capacité de nuisance en ralentissant le chantier. Le roman retrace les trajectoires de ces travailleurs de l'ombre : migrant clandestin passé par l'enfer des mines chinoises et les gargotes de chinatown, jeunes autochtones désœuvrés cherchant leur survie dans des emplois 
peu qualifiés, psychopathe asocial trouvant refuge dans cette solitude collective qu'est le chantier pour oublier ses crimes, mère de famille épuisée par les coups du sort et de son mari. Main-d'œuvre docile pour travail acharné. Au-dessus émerge une aristocratie du chantier : les ouvriers spécialisés et les ingénieurs. Ceux-ci sont dans la confidence de l'organisation. Ils sont, aux yeux des ouvriers, du côté des patrons, même si eux aussi triment dans le froid et la nuit. Le grutier, du haut de ses cinquante mètres, contredit ses complexes de petite taille, surplombe la rivière, le chantier, le pont et les hommes. La grue, c'est un métier noble dans le secteur du bâtiment où l'institution scolaire enferme les enfants d'immigrés et reproduit les inégalités sociales. Choisir la grue, c'est prendre de la hauteur sur soi, sur le chantier, sur les préjugés. La grue, c'est la porte ouverte à l'expatriation (à ne pas confondre avec émigration) pour rejoindre différents chantiers, dont celui, in fine, de Coca. La position instable du grutier de haut vol. Dans cet univers masculin, pour se faire une place et se faire accepter, les femmes se doivent d'accepter les pires conditions de travail, ne pas broncher, courber la tête, ne pas se laisser aller, (dé)montrer ses compétences et son intérêt pour des activités d'hommes. Telle l'ingénieure béton, dont les choix d'orientation sont orientés par les blessures d'une enfance où pour être élu, il vaut mieux être un garçon. Alors, ce sera des plaisirs de garçon (le foot, les jeux vidéo) et un travail de garçon (les travaux publics, le béton) au risque des railleries, des incompréhensions et de la solitude. Toujours, elle doit faire ses preuves, s'investir et se justifier : pourquoi le béton?

Malgré les manœuvres, les menaces et les cajoleries du maire, un tel chantier ne se fait pas sans heurter quelques intérêts. Certains n'hésitent pas à user de violence pour faire valoir leurs droits. Des habitants critiquent la mise en péril du patrimoine architectural, l'uniformisation des paysages urbains, le délogement des squatters et négocient l'octroi de nouveaux logements après avoir mobilisé les médias en leur faveur. Les ouvriers, craignant le non-paiement de leur prime, discutent directement avec le siège du consortium, à Bécon-les-Bruyères, dans une forme transnationale du conflit social, le décompte des pénalités de retard comme allié. Des entrepreneurs de transport fluvial, ruinés par ce concurrent de béton, tel un cartel mafieux, s'organisent pour saboter ce projet qui signifierait leur mort économique, eux qui jusqu'alors 
tenaient la ville entre leurs bacs et leur influence. Les autochtones voient leur mode de vie menacé par la déforestation promise par ce nouveau pont ; ou plutôt l'ethnologue, qui n'accepte pas de voir l'équilibre de sa propre existence (entre obligations professionnelles à l'université et séjours réguliers en forêt auprès de cette communauté) altéré par ce pont, sort de ses gonds et son couteau. Aucune de ces résistances n'obstrue la marche du chantier. Ce sont de simples oiseaux qui entraveront sa course et sa minutieuse organisation, en obligeant, via la mobilisation d'associations spécialisées et de la jurisprudence environnementale, à l'arrêt du chantier, de son bruit et de sa poussière, le temps de la nidification. Quelques semaines de chômage technique qui suspendent les routines et font tomber les pénalités. L'oiseau est le seul qui parvient (temporairement) à résister à cette entreprise titanesque, figurant la place de la nature dans le mouvement d'urbanisation : on la mate, elle résiste, on la fantasme, elle capitule, on la sanctuarise.

\section{Déterritorialisation}

Les deux piles du pont s'enfoncent dans les couches d'argiles sur lesquelles coule la rivière de Coca, mais leur construction révèle les processus de déterritorialisation à l'œuvre : circulation des modèles urbains, organisation transnationale de la maîtrise d'œuvre, migrations temporaires de travailleurs sur la route des chantiers.

Le pont de Coca n'est pas qu'un simple pont. Il est le grand projet du maire, le symbole de la puissance à venir, de la modernité comme mot d'ordre, des conflits d'intérêts comme combustible, de l'abus de pouvoir comme méthode. Ébloui par son séjour à Dubaï, archétype contemporain de la folie des grandeurs, des projets aussi coûteux qu'inutiles, de l'extravagance architecturale comme paravent du conservatisme social, politique et religieux, le maire lui aussi veut inscrire sa ville dans le circuit globalisé de l'économie, prendre sa part dans le gâteau mondial. Figure du maire bâtisseur, ambitieux pour sa ville et pour lui-même, le maire laissera sa marque par la transformation accélérée de la ville (qui dans le roman s'opère en deux ans) : muséification du centre historique, planification de nouveaux équipements et de nouveaux quartiers, développement d'une nouvelle filière économique d'écocarburant. L'infrastructure, le pont reliant les deux rives, est l'instrument de cette 
transformation, ouvre une nouvelle époque, symbolise le nouveau Coca. Le pont devient le prétexte de l'importation d'un modèle urbain globalisé et décontextualisé, que symbolise Dubaï, ville désincarnée, paradis de l'hyperlibéralisme, lieu de jouissance, de consommation, de richesses, construit par des travailleurs migrants sans protection juridique.

La promesse d'un chantier gigantesque agrège et attire à lui une foule motivée par le défi technique et la mise à l'épreuve de compétences professionnelles, par l'absence d'alternative personnelle et économique et l'espoir de gagner quelques mois dans une survie au quotidien, par le rêve d'une vie temporaire de pacha expatrié. On se déplace, on se met en mouvement, on se met hors de soi-même pour réaliser le pont, ouvrage qui relie deux rives et rassemble les mondes. Cette migration temporaire vers le chantier implique aussi des ruptures : avec l'univers routinier du quotidien, les amis, une mère trop présente, un meurtre qu' il conviendrait d'oublier... Au-delà du chantier de Coca apparaît une internationale des ouvrages monumentaux, à travers laquelle circulent des travailleurs : les ingénieurs pour leurs compétences, selon les contrats obtenus par leurs entreprises, des multinationales intervenant sur le marché global de la construction ; les ouvriers spécialisés auxquels on fait appel en soustraitance pour des tâches précises; les ouvriers, interchangeables, qui eux aussi traversent les continents pour rejoindre un nouveau chantier comme une nouvelle ruée vers l'or. Cette déterritorialisation du chantier est représentée par le personnage de l'ingénieur-chef, figure de l'expert hypermobile, apatride, dont les routines quotidiennes ne changent pas, quel que soit l'environnement de travail, comme un être désensibilisé aux territoires qu'il investit et transforme, tourné uniquement vers la matérialité de ce qu'il est sommé de produire, ici un pont, ailleurs un barrage, une mine, un centre commercial. Sa mission : résoudre les problèmes concrets de la production et faire jaillir l'objet tant convoité. Loin du chantier, au propre et au chaud dans les bureaux du siège à Bécon-les-Bruyères, ses collègues ingénieurs qui se sont acharnés pour remporter l'appel d'offres, subissant à échelle d'individu la compétition entre les firmes (le stress, les heures sup', l'attente, les insomnies), pour finalement ne pas faire partie des heureux élus de l'expatriation, tremplin professionnel, mais piège personnel. Les chantiers du bout 
du monde comme un eldorado ou comme un coup d'arrêt à la vie de famille. À l'inverse, les ouvriers traversent les continents sans certitude d'une embauche, comme un dernier espoir avant la chute.

Le chantier est un territoire temporaire, un espace de rencontre entre des mondes qui s'ignorent. Alors qu'un pont qui relie une rive à l'autre semble ancré dans un territoire et une réalité locale, le produire est un processus déterritorialisé, tant par les personnes qu'il mobilise que par la sociabilité éphémère de ces dernières. Le chantier déplace le monde, des ingénieurs les plus diplômés aux accidentés du travail et de la vie, rassemblés autour d'un pont et de leur solitude. Il crée un espace-temps singulier, un espace temporaire où se tissent les rencontres entre ces êtres solitaires, que les distances sociales et professionnelles du chantier entravent et menacent. Sociabilité de travail hors travail, travail qui absorbe les jours et les nuits. Les amitiés temporaires et viriles, l'idylle $\mathrm{du}$ patron et de l'ouvrière. Les solitudes se croisent. Cette solitude à double tranchant. Cette solitude qui isole et émancipe. Cette solitude qui colle à la peau malgré les enfants et le mari violent, malgré les responsabilités et les centaines d'hommes sous ses ordres. Cette solitude qui fait oublier comment on parle à une femme, comment on se tient devant un homme, comment on réfrène son désir. Que faire à Coca si ce n'est travailler, lorsque la grisaille de l'hiver et la solitude enferment dans une routine chantier-bowling-chantier ? Se jeter avidement sur cette brève parenthèse que promet la rencontre. Se donner la force de couper les ponts, de s'émanciper de la pression familiale. Se sentir vivre, un peu, au milieu de tout ce rien qu'est Coca, son pont, son chantier, ses motels minables, ses bars à cow-boys et sa violence sociale et humaine. Rendez-vous galant dans des fast-foods graisseux. Pas de temps pour les salamalecs. Sans espoir, sans mièvrerie.

Le pont prend forme, les grues surgissent, les caissons sont scellés, les câbles se tendent, le tablier relie enfin les deux rives. Les ingénieurs avaient bien calculé. Mais est-ce que ça tient ? Si l'on en croit certains acteurs du monde de l'ingénierie, ce roman serait « une excellente initiation au génie civil » ${ }^{1}$. À quoi ça tient ? Ce roman renvoie aux enjeux d'écriture des faits, scientifiques ou fictionnels, pour produire une représentation qui fasse sens pour celui qui la reçoit et engage à décrire pour expliquer

http://www.techniques-ingenieur.fr (consulté le 19 juillet 2017). 
la complexité du nouveau monde des infrastructures. Cette fiction très documentée mobilise en effet les moyens de la description pour produire un effet de réel qui fait tenir le pont et le roman, dont l'histoire est tendue par l'obligation de produire quelque chose (un pont), qu'il raconte de sa genèse (la prise de décision de construire le pont) à son inauguration.

\section{Références}

Baxandall M. (1991), Les formes de l'intention. Sur l'explication historique des tableaux, Paris, Éditions Jacqueline Chambon.

Becker H. S. (2009), Comment parler de la société : artistes, écrivains, chercheurs et représentations sociales, Paris, La Découverte.

Chérel E., Pasquier É. (2013), "La fiction et le réel ", Lieux communs, $\mathrm{n}^{\circ} 16$, p. 12-23.

De Kerangal M. (2010), Naissance d'un pont, Paris, Éditions Verticales.

Latour B. (2006), Changer de société, refaire de la sociologie, Paris, La Découverte.

Schaeffer J.-M. (1999), Pourquoi la fiction?, Paris, Seuil.

Vermandel F. (2013), "Quels enjeux épistémologiques pour la fiction ?", Lieux communs, $\mathrm{n}^{\circ} 16$, p. 29-44.

Interviews de Maylis de Kerangal :

http://rue89.nouvelobs.com/blog/cabinet-de-lecture/2010/08/24/maylisde-kerangal-naissance-dun-pont-linterview-integrale-163508

http://www.dailymotion.com/video/xe2mrx_maylis-de-kerangalnaissance-d-un-p_news 

INFRASTRUCTURES URBAINES :

LE PUBLIC ET LE PRIVÉ 



\title{
Conglomérats familiaux et infrastructures essentielles
}

\section{Le cas des Philippines}

\author{
Dominique Lorrain \\ Morgan Mouton
}

Une certaine conception de l'action collective explique l'organisation des villes en Occident. Elle repose sur une hiérarchie entre les catégories du public et du privé. À la première s'attache la notion d'intérêt général, à la seconde celle d'intérêts particuliers. Il est considéré que certaines activités touchant à l'intérêt général ne peuvent être totalement prises en charge par des entreprises privées. La fabrique de la ville, dans ses différentes composantes, en constitue une illustration exemplaire. Cette conception s'incarne d'abord dans la forme urbaine par une différenciation morphologique et juridique entre des espaces publics (rues, places, équipements) et des bâtiments privés. On la retrouve aussi pour l'organisation de l'espace : la définition des usages est une mission de puissance publique et relève de la planification urbaine. Enfin, cette conception est active pour l'organisation des infrastructures : activités essentielles sous la responsabilité des institutions publiques qui doivent être particulièrement réglementées si elles font appel à des entreprises privées ${ }^{1}$. Cela se traduit par des entreprises de services publics (utilities) régulées qui ne peuvent s'étendre à discrétion, car elles s'exposeraient à plusieurs critiques. La première relève de la philosophie politique : ces organisations privées ne sont pas légitimes pour exprimer l'intérêt général. La seconde vient de l'économie de la régulation : leur diversification serait facilitée par leur position de monopole dans leur activité principale et cela risque d'introduire une distorsion de concurrence. La troisième critique vient des marchés eux-mêmes où les

Services publics industriels et commerciaux, universal service (Matheu, 2002). 
analystes préferent évaluer des firmes spécialisées - pure players - plutôt que des grandes firmes diversifiées et plus complexes. L'évolution des compagnies d'eau britanniques, de Veolia et de Suez en France, des électriciens allemands - Eon et RWE, depuis le milieu des années 1990 - témoigne de la force de ces principes.

Sur ces questions, l'Asie en pleine expansion nous donne à voir quelque chose de très différent. L'économie dans plusieurs pays s'y trouve dominée par quelques conglomérats familiaux ; la plupart d'entre eux ont développé des activités urbaines et quelques-uns interviennent principalement sur le marché de la fabrique urbaine. La puissance publique délègue globalement la réalisation de nouveaux morceaux de villes à ces grands conglomérats familiaux qui prennent en charge conception, financement, construction et gestion ${ }^{2}$. Les villes mélangent des espaces publics et privés à l'intérieur de complexes intégrés (integrated resorts) et de centres commerciaux géants. Ce sont ces espaces urbains particuliers qui nous intéressent, dans lesquels le bâti et les services en réseaux sont pensés comme des actifs (fixed assets) par les grands conglomérats familiaux des pays d'Asie qui les produisent.

Un conglomérat peut se définir comme une organisation souple dans laquelle des sociétés opérant dans des secteurs différents sont rattachées à une holding de tête. Ce type de firme ne tire pas sa force d'une intégration industrielle, mais d'une capacité à déceler des secteurs porteurs, d'y entrer, et d'en sortir lorsque les conditions ne sont plus remplies. Ainsi, Anthony Salim, président du groupe éponyme, plus puissant conglomérat d'Asie au milieu des années 1990, expliquait à un journaliste du Asian Wall Street Journal : "The group isn't driven by a particular product or technology $[. .$.$] we are opportuny driven { }^{3}$. " Dans cette partie du monde, les conglomérats ont des activités dans les matières premières, la banque, ils détiennent des franchises de distribution pour des groupes étrangers. Ils interviennent dans l'industrie, parfois, et surtout ils sont très présents dans tout ce qui concerne la fabrique urbaine : promotion, construction, hôtellerie et loisirs, infrastructures de transport, services publics (électricité, eaux, télécommunications). On les trouve à Hong Kong, en Malaisie, en Indonésie, aux Philippines, en Corée du Sud et dans une forme plus publique à Singapour et en Chine. Ces conglomérats sont puissants et

2 Les contrats utilisés ne relèvent pas des concessions françaises, mais de schémas mis au point aux États-Unis de type DBFO (design, build, finance, operate).

3 Asian Wall Street Journal, 28 février 1996, p. 6. 
très connectés à la classe politique ${ }^{4}$. Ce qui serait dénoncé en Occident comme relevant de la corruption, de la « capture » et du conflit d'intérêts semble avoir peu d'écho dans cette partie du monde. Les universitaires, les oppositions politiques et la presse semblent majoritairement accepter ces situations. Dans ces conditions, quels sont les fondements intellectuels religieux, philosophiques, politiques - qui rendent acceptable pour le plus grand nombre cette organisation des pouvoirs?

Nous proposons d'explorer cette question sur les fondements de l'action publique urbaine déléguée en bloc, par une double démarche : une présentation des activités urbaines de ces conglomérats aux Philippines afin d'en démontrer l'importance ${ }^{5}$; une réflexion sur les catégories de l'action et la conception des liens entre public et privé. En insistant sur l'histoire des Philippines et de ses élites, l'enjeu sera également de comprendre pourquoi la présence de ces conglomérats n'est pas remise en question.

\section{Ce que font les conglomérats des Philippines dans la fabrique urbaine}

Un rapide examen de la littérature, comme le suivi de la presse économique, permettent de constater la très grande concentration de l'activité économique autour de quelques conglomérats aux Philippines (Hutchcroft, 1991 ; Krinks, 2003 ; Bello, 2005). En partant de ce constat, il apparaît intéressant d'entrer dans l'économie politique du pays par les grandes familles qui tiennent ces conglomérats. C'est ce que propose notamment l'historien Alfred McCoy (1993). Certains de ces groupes sont "installés " et dirigés par une même famille depuis plusieurs générations. Leur histoire se confond avec celle de ce pays. Certains affirment même avoir une responsabilité ou une ambition plus grande que celle de simplement faire des affaires. Relèvent de ce premier ensemble de conglomérats historiques : Ayala Corp. (1834, famille Zobel de Ayala) ; Lopez Group (1903, famille Lopez) ; San Miguel (1891,

Sur la question des liens avec la classe politique, voir, dans le cas des Philippines, Hutchcroft (1991), Kan (2002), McCoy (2009).

5 Ce travail est pour une part documenté à partir de « portraits d'entreprises " : Ayala et Salim. Voir: http://chaire-ville.enpc.fr/les-portraits-dentreprises (consulté le 10 juillet 2017). 
famille Soriana et Cojuangco, diversifié dans les infrastructures) ; Aboitiz Group (1901, famille Aboitiz). Depuis les années 1980, de nouveaux groupes ont émergé, souvent issus de la communauté chinoise. Ils peuvent être considérés comme des challengers, mais cette concurrence oligopolistique s'inscrit dans un marché en forte croissance et surtout ils reproduisent le modèle fondateur du conglomérat familial actif dans la fabrique urbaine. On peut citer notamment SM Prime Holdings (1985, Henry Sy le « roi » des malls); J.G. Summit (1957, John Gokongwei) qui contrôle Robinsons Land Corp., très actif dans les centres commerciaux, ainsi que la compagnie aérienne Cebu Pacific ; Filinvest Development Corp. (1973, famille Gotianun) ; Metro Pacific (1982, famille Salim, Indonésie) ; Megaworld Corp. qui a développé le CBD d'Eastwood.

\section{Les principaux conglomérats aux Philippines}

\begin{tabular}{|c|c|c|c|}
\hline Nom & Famille & $\begin{array}{l}\text { Chiffre } \\
\text { d'affaires } \\
\text { (en 2013, en } \\
\text { USD) } \\
\end{array}$ & Secteurs d'activités \\
\hline San Miguel & $\begin{array}{l}\text { Soriano puis } \\
\text { Cojuancgo }\end{array}$ & $16,6 \mathrm{G} \$$ & $\begin{array}{l}\text { Pétrochimie ; agroalimentaire ; } \\
\text { emballage et conditionnement ; } \\
\text { énergie ; infrastructures de transport }\end{array}$ \\
\hline Ayala Corp. & $\begin{array}{l}\text { Zobel de } \\
\text { Ayala }\end{array}$ & $3,6 \mathrm{G} \$$ & $\begin{array}{l}\text { Banque ; promotion immobilière ; } \\
\text { eau et assainissement ; } \\
\text { télécommunications ; transports } \\
\text { urbains ; automobile ; BPO }\end{array}$ \\
\hline JG Summit & Gokongwei & $3,3 \mathrm{G} \$$ & $\begin{array}{l}\text { Agroalimentaire; transport aérien ; } \\
\text { promotion immobilière ; banque ; } \\
\text { pétrochimie }\end{array}$ \\
\hline $\begin{array}{l}\text { SM } \\
\text { Investments }\end{array}$ & Sy & $2,2 \mathrm{G} \$$ & $\begin{array}{l}\text { Grande distribution ; promotion } \\
\text { immobilière ; banque }\end{array}$ \\
\hline $\begin{array}{l}\text { Lopez Group } \\
\text { (anciennement } \\
\text { Benpres } \\
\text { Group) }\end{array}$ & Lopez & $2,1 \mathrm{G} \$$ & Énergie ; médias \\
\hline Aboitiz Group & Aboitiz & $2 \mathrm{G} \$$ & $\begin{array}{l}\text { Énergie ; banque ; agroalimentaire ; } \\
\text { promotion immobilière }\end{array}$ \\
\hline $\begin{array}{l}\text { Megaworld } \\
\text { Corp. }\end{array}$ & Tan & $0,8 \mathrm{G} \$$ & Promotion immobilière \\
\hline
\end{tabular}

Source : auteurs, d'après les rapports annuels des groupes pour l'année 2013. 
L'activité des conglomérats aux Philippines a été durablement impactée par le développement du secteur immobilier dans les grandes villes. Cette manne de croissance a créé des opportunités pour les grands groupes : entre 1986 et 1996, les ventes immobilières augmentent en volume de $15 \%$ par an en moyenne, tandis que les prix augmentent annuellement de $20 \%$ en moyenne (Magno-Ballesteros, 2000). À Manille, l'index immobilier, qui pendant longtemps représentait $20 \%$ de l'indice composite de la place, a crû vivement pour s'établir à $35 \%$ à l'automne 1996, après les fortes hausses de l'immobilier commercial centres commerciaux, bureaux ${ }^{6}$. Cet essor du secteur immobilier a été polarisé par des centres d'activité (Central Business Districts, CBD) qui donnent à voir un paysage urbain qui se démarque nettement du reste de la ville. Plusieurs des conglomérats cités se sont diversifiés pour intervenir dans toutes les composantes de la fabrique urbaine. Ainsi le groupe Salim, par ses filiales First Pacific (Hong Kong) et Metro Pacific Investment (Manille), gère aux Philippines plusieurs biens essentiels : l'électricité, le téléphone fixe et mobile, l'eau, les autoroutes à péage, les hôpitaux, plusieurs journaux et une chaîne de télévision (Tiglao, 2014 ; Lorrain, 2016). Il ne lui manque que les activités de développement urbain et de promotion pour être un groupe urbain total. Cette capacité " totale » est aujourd'hui la mieux représentée par le groupe Ayala et par son engagement dans les quartiers centraux de Manille (Lorrain et Mouton, 2017) ; si l'on traduit cela dans des codes français, ce serait comme si le baron Haussmann et la Compagnie Générale des Eaux avaient fusionné leurs intérêts et modelaient le centre de Paris. Le quartier de Makati, développé dès les années 1960 par la famille Zobel de Ayala, est un modèle du genre. C'est le premier quartier dans lequel des normes urbanistiques strictes sont imposées avec succès (hauteur des immeubles, zonage, etc.) et qui a fait l'objet d'une planification en amont, permettant d'anticiper les questions de densité urbaine, de circulation routière, etc. (Kishiue, 2000). Un tel modèle de développement repose entièrement sur le secteur privé : ici, Ayala Land, propriétaire du foncier, assure le ramassage des déchets, la desserte en eau ou encore la sécurité. Par la suite, le quartier d'Ortigas et plus récemment le quartier de Fort Bonifacio ont suivi un mode de développement similaire. Le contraste avec le reste du tissu urbain est saisissant : la propreté des rues et la verticalité du bâti rendent ces espaces reconnaissables. Ils

Financial Times, 13 septembre 1996. 
accueillent des entreprises étrangères (de $\mathrm{BPO}^{7}$ notamment), des centres commerciaux gigantesques ou encore des commerces franchisés. Leur intensité capitalistique les rend distincts du reste de la ville.

\section{Exemples de grandes opérations déléguées en bloc}

Fort Bonifacio est à l'origine une ancienne base militaire américaine localisée dans Manille que l'État, représenté par Bases Conversion and Development Authority (BCDA), veut développer. Il s'agit d'un projet de grande ampleur : 214 ha dans le prolongement du quartier des affaires de Makati, en voie de saturation. Les mots utilisés pour le désigner témoignent de cette ambition : the Global City, the city within the city, the first 21st century city. Cet espace est conçu pour offrir une grande qualité de service. Comme dans les parcs industriels promus en Chine par la ville État de Singapour, un effort particulier est prévu dans les infrastructures qui précèdent les constructions et dans la planification spatiale : tracé des rues, qualité des réseaux d'environnement, interdiction des transports collectifs populaires (jeepneys et tricycles à moteurs) au profit de systèmes de transport locaux électriques.

À son lancement ce projet est prévu sur 25 ans (1995-2020). Une jointventure est constituée - Fort Bonifacio Development Corporation - entre BCDA/ le gouvernement $(45 \%)$ et un consortium privé "Bonifacio Land Corporation " (55\%). Un appel d'offres a lieu en janvier 1995 pour sélectionner le partenaire privé. En sort vainqueur le consortium dirigé par Metro Pacific, filiale de First Pacific, qui a fait une offre de $36 \%$ supérieure à la seconde offre déposée par le groupe Ayala. Dans ce consortium, on trouve un poids lourd de l'immobilier commercial, Henry Sy, et sa société, SM Prime Holdings. En 1995, les anticipations sont totalement positives. Mais les marchés vont enregistrer deux chocs - la crise asiatique de l'été 1997, la crise des dotcom à la fin 2001 -, qui remettent en cause les épures. Le groupe Salim, maison mère de First Pacific qui commande Metro Pacific est touché de plein fouet. Il doit vendre des actifs pour éviter la banqueroute. Après différentes tractations qui impliquent le groupe J.G. Summit, c'est finalement le groupe Ayala qui reprend la participation de Metro Pacific dans Bonifacio Land.

Ce cas est instructif à un double titre. Il renseigne sur la manière dont se conçoit, se finance et se réalise l'urbanisation privée à grande échelle. Il nous donne à voir aussi la compétition et les arrangements entre les grands conglomérats pour accéder aux opérations stratégiques.

L'acronyme désigne le secteur de la sous-traitance de services (Business Process Outsourcing). 
L'aménagement et la poldérisation (réclamation) partielle de la baie de Manille, qui à terme fera émerger un CBD développé sur un espace de 600 ha. SM Prime Holdings y a installé un complexe comprenant l'un des plus grands malls du monde, ainsi qu'un centre des congrès (Guéguen, 2013). Ce conglomérat vient également de remporter un projet de poldérisation qui s'inscrit dans cette volonté de développer un nouveau centre économique sur la baie de Manille. SM Prime Holdings est intéressant à plus d'un titre. Il développe la plupart des malls importants dans le pays, et il est devenu un promoteur majeur dans la capitale. C'est aussi un conglomérat récent, créé par un membre de la communauté chinoise, M. Henry Sy, né en 1924 et aujourd'hui l'homme le plus riche des Philippines. Il diversifie graduellement ses activités autour de la fabrique urbaine : urbanisme commercial, promotion immobilière et casinos. C'est un modèle qui diffère un peu des conglomérats familiaux traditionnels plus éclectiques dans leur portefeuille. L'enjeu serait donc d'observer si des différences existent dans la façon dont ces deux types de conglomérats envisagent la production urbaine.

\section{Ce que nous enseigne le détour par l'histoire en Occident}

La question principale que pose une telle concentration de l'activité économique aux mains d'une poignée d'acteurs privés est celle de l'indépendance de la décision publique avec son revers de la corruption. Le problème est d'ailleurs au centre du débat politique aux Philippines puisque le président Benigno Aquino s'est fait élire en 2010 avec le slogan "Sans corruption et pas de pauvreté " (Kung walang corrupt, walang mahirap). L'un de ses premiers actes a été de former une commission (truth commission) chargée d'enquêter sur les multiples allégations de corruption mettant en cause les neuf années de mandat de son prédécesseur - Gloria Macapagal-Arroyo. Son successeur, le très populiste Rodrigo Dutertre, élu en mai 2016, a quant à lui mis en avant une image "anti-establishment $\|^{8}$ et, dans le cadre de sa campagne, a désigné nommément un certain nombre d'administrations qu'il jugeait corrompues en promettant de les réformer. Pour éclairer ces réflexions contemporaines sur le positionnement de l'État philippin et de la société civile par rapport aux pratiques de corruption courantes

8 Lors d'une allocution durant la campagne électorale, il s'est ainsi adressé à l'élite du pays avec les mots suivants : « When I become president, by the grace of God, I serve the people, not you» (Daily Mail, 30 juin 2016). 
dans le pays, il n'est pas sans intérêt de faire un détour par les pays d'Europe occidentale où le problème se posa avec force. Comment s'y sont construites la notion d'intérêt général et, par là même, les normes de probité qui organisent les pratiques?

Au départ, les sociétés occidentales s'organisent à partir d'une loi divine ou loi naturelle qui en surplomb guide les comportements (Chevallier, 1966 ; Spitz, 1994). Le grand mouvement des idées qui traverse les $\mathrm{XVII}^{\mathrm{e}}$ et $\mathrm{XVIII}^{\mathrm{e}}$ siècles se caractérise par un phénomène de sécularisation et d'affirmation des droits des individus. Ce fut une combinaison de questionnements portés par des penseurs dont quelquesuns sont passés à la postérité - Hobbes 1651, Locke 1689, Montesquieu 1748, Rousseau 1762 - et de moments révolutionnaires en Angleterre et en France. Cependant, en détachant les individus des liens qui les tenaient - Christopher Hill, qui étudie la révolution de Cromwell, parle de masterless men (Hill, 1972, p. 32-56) -, la société s'expose au risque du chaos, résumé par la formule " la guerre de tous contre tous " (Hobbes, 1983). La réponse à ce problème a conduit à concevoir un ordre supérieur aux individus, qui ne leur est plus extérieur et imposé, comme la loi naturelle/divine, mais le produit de leur consentement. Les citoyens définissent un intérêt général représenté par des institutions publiques au premier rang desquelles on trouve l'État. Celui-ci se constitue selon deux formes avec la figure autoritaire du Léviathan dessinée par Hobbes, ou l'autorité démocratique du Parlement dont discute Locke prolongé, par la théorie de la séparation des pouvoirs de Montesquieu. Au-delà des différences dans les solutions proposées, ces pensées partagent plusieurs principes : premièrement la reconnaissance d'un état de nature comme point de départ; deuxièmement l'affirmation d'un pouvoir des individus qui, pour ne pas sombrer dans le chaos, délèguent à un pouvoir supérieur ; ce peut être le Parlement ou l'homme artificiel qu'est le Léviathan, pour reprendre l'expression de JeanJacques Chevallier (1966, p. 52) ; troisièmement cette opération de délégation de pouvoir des individus à un être collectif supérieur conduit à introduire une différence hiérarchisée entre les intérêts particuliers et l'intérêt général. Il y a une longue filiation intellectuelle et pratique qui débute avec la révolution anglaise de 1642-1653, court tout au long du $\mathrm{XVIII}^{\mathrm{e}}$ siècle avec les Lumières, culmine avec la Révolution française et l'indépendance des États-Unis et se poursuit pendant une partie du 
$\mathrm{XIX}^{\mathrm{e}}$ siècle, pour que le principe de sécularisation soit définitivement installé dans les affaires publiques. Ce long travail d'invention de catégories politiques commence par détacher les individus du poids de la religion, se poursuit en affirmant la nécessité de les organiser à partir d'un projet collectif rationnel ${ }^{9}$ qui dépasse les intérêts particuliers pour affirmer un intérêt général.

Poursuivons avec la corruption comme retour des intérêts particuliers et dévoiement de l'intérêt général. Et suivons un instant l'argumentation de Robert Neild ${ }^{10}$ qui part d'un constat réaliste : "If you look at history and across the world today [...] it is uncorrupt government that is exceptional " (Neild, 2002, p. 1), un constat partagé quelques années plus tard par North, Wallis et Weingart (2010). Au cœur de ces pratiques, on trouve les conceptions de l'intérêt général et des intérêts particuliers; pendant longtemps ils se confondent. À partir de là, deux grandes interrogations peuvent être déployées. Qu'est-ce qui rend acceptable dans certains pays cette confusion de ces catégories du public et du privé ? Dans d'autres pays, comment s'est opérée la sortie de l'ordre existant, et quelles sont les raisons de la mise au point d'un nouveau régime d'action fondé sur une séparation entre l'intérêt général et les intérêts particuliers?

À la fin du XIX ${ }^{\mathrm{e}}$ siècle, quelques pays occidentaux mettent en cause les pratiques de corruption pour élaborer de nouvelles règles. Neild souligne en premier la concurrence militaire comme facteur puissant pour rechercher l'efficacité dans l'action de gouverner ${ }^{11}$. La conduite de la guerre nécessite des moyens importants et cela pousse les États à rechercher l'efficacité : ce sont les cas de la Grande-Bretagne, de la France, de la Prusse et des États-Unis. Il étudie comment les normes ont évolué dans ces pays aux XVIII ${ }^{\mathrm{e}}$ et $\mathrm{XIX}^{\mathrm{e}}$ siècles.

"Military competition between nations has favoured the expansion of the less corrupt vis-à-vis the more corrupt: those nations that have developed efficient, relatively uncorrupt government have been able to mobilize their resources $[\ldots]$ more effectively than the more corrupt $[\ldots]$. In short, war

9 Pour une autre conception de la rationalité voir, pour la Chine, Jullien (1998).

10 Robert Neild (2002) a occupé des fonctions universitaires - à Cambridge -, mais également des postes importants dans l'administration à Whitehall et à l'étranger Inde, Nouvelle-Guinée, Nations unies.

11 Voir les déductions à partir des travaux de Ruciman (Neild, 2002, p. 14). 
has been a significant, albeit brutal, test of government efficiency and has thereby been a selective force favouring the more efficient, which as a rule are the less corrupt " (Neild, 2002, p. 14). Dans le long mouvement qui va vers des règles impersonnelles et justes, il retient ensuite l'instauration d'une justice indépendante.

Une deuxième série de facteurs explicatifs peut se résumer dans la formule "trop c'est trop " : à un certain niveau, la corruption n'est plus acceptable, elle fait peser un fardeau trop lourd sur la société. Des scandales éclatent; ils font ressortir des liens personnels entre industriels et hommes politiques qui conduisent à des surfacturations, à des hausses tarifaires injustifiées. On relèvera qu'au départ la dénonciation de ces pratiques vient rarement de ceux qui sont dans le système, mais d'acteurs en périphérie : des journalistes et quelques universitaires. Le cas emblématique est celui des États-Unis, où se développe à la fin du XIX siècle une critique radicale des machines politiques, du clientélisme et de la corruption (Banfield et Wilson, 1966 ; Lowi, 1969 ; Tarr, 1989 ; Bonnet, 2010 ; Matheu, 2002). Elle débouchera sur la mise en place d'un ensemble de réformes qui font entrer l'action publique urbaine dans la modernité telle que nous la connaissons : professionnalisation des emplois (city manager), mise en place de la planification urbaine, création des special district pour gérer certaines activités et de commissions de régulation pour les utilities. Dans un registre plus monographique, l'histoire du scandale du gaz à Salford, rapportée par John Garrard (Garrard, 1992 ; Defeuilley, 2013 ; Lorrain 2000), rappelle que le versement de commissions au bénéfice personnel des acheteurs relevait au milieu du XIX ${ }^{\mathrm{e}}$ siècle des pratiques commerciales habituelles dans de nombreuses branches de l'industrie britannique et que ces pratiques s'étaient diffusées dans les gouvernements locaux. Ce cas est instructif également par l'éclairage fin qu'il porte sur les facteurs de possibilité ; ils tiennent en un constat : la faible structuration organisationnelle des gouvernements urbains. Les élus exerçaient leur mandat tout en poursuivant leur activité professionnelle ; pour la plupart d'entre eux, il s'agissait d'une " charge » au sens littéral du terme et dans l'esprit de l'époque il n'était pas anormal qu'elle soit assortie d'un dédommagement. Neild observe en écho : "It was normal practice for government ministers in England in the eighteenth century to lend out the public funds belonging to their departments and pocket the 
interest " (Neild, 2002, p. 1). Les services municipaux eux-mêmes étaient peu structurés et leurs règles d'action imparfaitement élaborées ; l'ensemble reposait sur des relations interpersonnelles entre les élus, les chefs de services, les fournisseurs et la population. C'est dans ces jeux de services rendus et d'estime interpersonnelle que venaient se loger des pratiques de corruption, nommées ainsi si on les évalue à l'aune de règles fondées sur la probité. Pour les contemporains, tant que ces échanges ne franchissaient pas certaines limites, ils n'étaient pas répréhensibles.

Puisque ces règles de probité ne sont pas universelles, il convient de s'interroger sur les mécanismes de corruption dans les pays où elle perdure et sur les facteurs d'acceptation. À nouveau, le détour par le $\mathrm{XIX}^{\mathrm{e}}$ siècle s'avère instructif, car il tend d'abord à montrer que ces pratiques sont acceptées, car tout le monde y trouve un intérêt : les pratiques de l'industrie en Grande-Bretagne au milieu du XIX ${ }^{\mathrm{e}}$ siècle, ou le fonctionnement des " machines politiques " comme pourvoyeur d'emplois et de services. Évidemment, tout le monde n'en bénéficie pas dans les mêmes proportions. Si la corruption se perçoit comme un dispositif de prélèvement sur la richesse produite, ou à produire, alors il y a de gros tuyaux de dérivation et de plus petits. Mais ici, l'important tient moins à la taille qu'au partage d'une commission, d'un emploi ou d'une rente ; leur diffusion généralisée sape la légitimité de toute critique. Une autre explication du maintien de l'existant tient au comportement des élites privées qui rendent acceptables leurs pratiques malgré l'immensité de leurs pouvoirs. Neild nous livre le cas très intéressant de la Compagnie des Indes orientales britannique qui donne à comprendre, avant la mise en place de règles, les formes de la corruption et les ressorts d'un gouvernement vertueux si l'on se demande pourquoi les directeurs de la Compagnie n'abusaient pas de leur pouvoir à leur seul bénéfice personnel. Selon un auteur dont il rapporte le propos, le credo des politiciens de l'époque et des directeurs de la Compagnie était de faire une fortune confortable dans le service public et de placer ses proches en situation de profit. "But the results of this attitude were not as desintegrating as might have been expected. This was chiefly because the ambitions of such men were kept within the bounds of moderation by the dominance of a wealthy aristrocracy " (Sutherland, cité par Neild, 2002, p. 76). Cette modération trouvait ses racines dans la richesse établie de ce groupe social dirigeant et dans les buts supérieurs qu'il 
se fixait en tant que classe dirigeante. "In the last resort [they] felt some responsibility for, and interest in, the maintenance of the king's government and in the prosperity and prestige of the country " (Neild, 2002, p. 76) La citation se poursuit en expliquant comment, au sein de la Compagnie et de l'État, fonctionne l'équilibre entre intérêts public et privé. "A man should if he survived ${ }^{12}$ make a considerable fortune in the Company's service, but only if he had worked his twelve or fifteen years through the ranks and given the Company the benefits if his industry and acquired experience. " Au milieu du XVIII ${ }^{\mathrm{e}}$ siècle en Inde, la chute de l'Empire moghol, les victoires sur les Français et sur plusieurs royaumes indépendants conduisent à une dégradation des pratiques, car les possibilités d'enrichissement se trouvent démultipliées. "The opportunities for enrichment were so tempting that the company's servants cast aside restraint. [...] The Company's servants having gained a suddent affluence, became "quite unmanageable" " (Neild, 2002, p. 76), propos venant d'un grand directeur de la Compagnie. Autre manière de dire avec la citation d'un autre auteur : "In the early days the Company had been content with trading privileges but, with the dissolution of the Mogul empire, it began to trade in kingdoms, seeking profit in tribute rather than in commerce" (Neild, 2002, p. 77).

Autrement dit, dans une configuration se caractérisant par de faibles règles formelles et par des pouvoirs importants des cadres de la Compagnie, doublés d'une grande liberté en ces territoires éloignés du centre de l'Empire, l'explication première de leurs comportements tient aux valeurs. La limitation informelle à la cupidité, ce qui maintient les comportements dans les limites de l'acceptable contrairement à ce qui pouvait être attendu, trouve ses causes dans la nature de l'élite et dans les principes supérieurs auxquels elle adhère. Mais ces équilibres sont fragiles. Les comportements ne suivent pas des règles accompagnées de sanctions, tout à l'inverse ils reposent sur l'adhésion des personnes et sur un subtil équilibre entre le service de la Compagnie et les intérêts privés. Dans ces conditions, les situations de crises mettent en péril ces équilibres : de nouvelles personnes font leur entrée sans nécessairement avoir intégré tous les principes informels. Les équilibres de pouvoir changent, le temps s'accélère et, lorsque des fortunes peuvent se faire très vite, les barrières morales sautent.

12 Le taux de mortalité était alors élevé dans ces voyages aux Indes. 


\section{État faible et élites privées installées}

L'un des éléments notables aux Philippines, et qui a sans doute facilité le développement d'un État rentier en proie à des pratiques très généralisées de corruption, tient au processus de constitution des élites. Ce pays a été dominé tour à tour par les Espagnols, puis les Américains. Depuis l'indépendance en 1946, il a connu la loi martiale de 1972 à 1986, date d'une révolution populaire pour démettre le président Marcos. Ensuite six présidents se sont succédé, l'un d'entre eux fut destitué en 2001 par une procédure d'impeachment pour fait de corruption. Une caractéristique de long terme semble donc être celle d'une faiblesse de la classe politique et des institutions publiques. A cette instabilité de l'État répond la grande continuité des dynasties privées, puissantes, qui se considèrent comme dépositaires des intérêts supérieurs de la Nation. Depuis la période de colonisation espagnole, de grandes familles de propriétaires terriens qui entretiennent de bonnes relations avec l'occupant se partagent le pouvoir économique. L'arrivée des Américains en 1898 ne modifie pas cette situation et permet même à ces acteurs économiques d'acquérir une dimension politique en introduisant un système de représentation qui favorise l'émergence de pratiques de patronage. Les élections de 1907 confirment le rôle des élites économiques à travers tout le pays (Hutchcoft, 2008). Après l'indépendance, un tel système n'est pas remis en question et on voit se développer des pratiques que l'on peut rapporter au modèle du patrimonialisme ${ }^{13}$ construit par Max Weber : les affaires d'État sont gérées comme des affaires personnelles, et la politique est conduite en fonction des intérêts d'oligarques proches des décideurs politiques (Hutchcroft, 1991). On peut illustrer cette tendance avec la question de la réforme agraire toujours évoquée, car relevant de la nécessité économique pour le pays, et toujours reportée, car elle va à l'encontre des intérêts des grandes familles de l'élite. Ce refus de procéder à une

13 "In the genuinely patrimonial office, the political administration... is treated as a purely personal affair of the ruler, and political power is considered part of his personal property " (Max Weber, Economy and Society, 1921, cité par Hutchcroft, 1991, p. 415). Par opposition à une administration " moderne " qui fonctionne sur des bases légales, l'administration patrimoniale repose sur des relations personnelles de subordination entre les agents. 
réforme est une constante de la vie politique depuis l'indépendance du pays, chaque président promettant de s'y atteler sans pour autant, une fois élu, modifier la situation de manière significative (Bello, 2005).

Cette relation entre les élites économiques du pays et le pouvoir politique oriente ainsi l'action publique depuis longtemps. Certes, le degré d'influence et l'insertion des grandes familles dans l'appareil d'État sont variables ${ }^{14}$, mais on peut généralement affirmer que les pratiques de prédation des ressources publiques ont eu un impact certain sur la trajectoire économique du pays. Si les pratiques de prédation et les divers avantages accordés aux grands conglomérats familiaux ont pu être critiqués (Hutchroft, 1991 ; World Bank, 1992 ; Bello, 2005), l'existence des grands groupes aux Philippines n'est pas elle-même remise en question. Comme le fait Kang (2002), dans son ouvrage qui se propose d'expliciter les liens entre pratiques rentières et développement sur la base d'une comparaison entre les Philippines et la Corée du Sud, on peut faire l'histoire des relations entre ces grandes familles et l'appareil étatique. L'auteur distingue plusieurs périodes. Suite à l'indépendance du pays, le rapport entre l'État et les élites économiques est très inégal, avec un État faible qui se fait phagocyter par les familles influentes du pays. Sous le régime de Marcos, un discours qui condamne les pratiques rentières et l'existence d'oligarques est mis en avant, mais plus qu'une remise en question du modèle patrimonial, c'est une centralisation qui s'opère ; il y a moins d'oligarques, mais qui ont chacun plus d'influence qu'auparavant). Kang voit cependant, après la chute du dictateur, les prémices d'un changement structurel plus favorable au développement du pays et l'émergence d'une économie qui ne repose plus sur le népotisme.

La faiblesse d'un État philippin en proie aux pratiques rentières des élites économiques du pays peut être attribuée aux expériences coloniales qu'a connues le pays (McCoy, 1993). L'Espagne puis les États-Unis ont tenté de mettre en place un appareil bureaucratique en transposant leurs propres lois et coutumes sur l'archipel. La population, se voyant imposer

14 On observe des différences significatives dans l'implication des familles dans l'appareil d'État. On peut citer par exemple le cas de la famille Lopez, qui a très tôt investi l'administration publique et dont plusieurs membres ont occupé des fonctions au sommet de l'État, par opposition à la famille Zobel de Ayala, restée plus distante par rapport à la classe politique. 
par le haut un ensemble d'institutions étrangères à ses propres traditions, a fait preuve de défiance et les autorités coloniales ont dû asseoir leur autorité par la coercition. La puissance américaine a très tôt identifié le risque que posaient les élites provinciales philippines - surnommées les caciques - et a mis en place une police politique (Philippine Constabulary) afin de contrôler leurs abus de pouvoir et leurs pratiques rentières. À la suite de l'indépendance du pays en 1946, l'administration doit à son tour œuvrer pour contenir l'appétit des élites provinciales et des oligarques du pays. Cependant, alors que le gouverneur qui dirigeait le pays était auparavant nommé par Washington, le président philippin est issu d'un scrutin quil'oblige à gagner les faveurs des notables locaux pour remporter l'élection. On observe par ailleurs une dégradation du fonctionnement de l'administration publique, qui sous la tutelle américaine faisait preuve d'intégrité et d'efficacité. L'économiste et historien Onofre D. Corpuz dépeint l'administration d'après-guerre ainsi : " [It] was characterized mainly by low prestige, incompetence, meager resources, and a large measure of cynical corruption ${ }^{15}$. " Parallèlement, le poids de l'État philippin sur l'économie se fait de plus en plus important. Les fonds américains et japonais, en réparation des dommages causés par la Seconde Guerre mondiale, alimentent l'emprise du gouvernement central sur l'économie et attisent les convoitises des oligarques. L'État a une emprise sur l'économie qui se fait de plus en plus grande, mais il s'avère incapable de mener à bien une politique de développement. Cette combinaison de ressources économiques importantes et d'une faiblesse de son administration rend l'État particulièrement vulnérable aux pratiques rentières du personnel politique.

\section{L'acceptabilité des missions publiques assurées par les conglomérats philippins}

L'omniprésence de grands groupes privés dans des activités qui en Occident relèvent d'une maîtrise publique, même si elles sont déléguées ensuite, ne semble donc pas soulever d'oppositions franches dans le contexte philippin. Ainsi, lors d'un entretien avec le Business Times de

15 Propos tirés de l'ouvrage de McCoy (1993, p. 12). 
Singapour, l'un des dirigeants du groupe Ayala Land, Inc. insiste sur ce rôle de quasi-substitut à l'État que prend son entreprise lorsqu'elle développe des quartiers entiers :

"The fact that there is nobody in the Philippines who regulates urban planning has been great for Ayala Land, because we are probably the only company there that has the scale financially to take on large plots of land. [...] By developing big tracts of land, we become the government; we control and manage everything. We are the mayors and the governors of the communities that we develop and we do not relinquish this responsibility to the government. [...] But because we develop all the roads, water and sewer systems, and provide infrastructure for power, we manage security, we do garbage collection, we paint every pedestrian crossing and change every light bulb in the streets - the effect of that is how property prices have moved ${ }^{16}$. "

Une autre illustration de ce positionnement aux franges du marché et de missions publiques nous est donnée avec la famille Lopez. C'est une des grandes familles de l'élite du pays dont les membres ont occupé des positions importantes dans l'appareil d'État ${ }^{17}$. Dans le monde des affaires, ils étaient connus par leur filiale cotée, Benpres, en charge de la distribution électrique dans la région métropolitaine de Manille et des eaux urbaines dans une moitié de ce territoire. En 2002, après la crise asiatique de 1997, puis le retournement du marché des dotcoms, la fête est finie. Le groupe Benpres et ses filiales accumulent les dettes. Des critiques s'élèvent et appellent à un changement de paradigme : le groupe doit se remettre en cause et ne plus se penser comme incarnant la Nation, mais tout simplement comme une entreprise de services. Lorsqu'il s'adresse à ses actionnaires en 2002, Oscar Lopez (Chairman et $\mathrm{CEO}$ ) refuse un tel changement de paradigme : "When Benpres was formed in $1993^{18}$ its mission was to preserve and continue the Lopez business philosophy, namely the provision of high-quality public services to the Filipino people " (FEER, 15 août 2002, p. 38-42, voir aussi Mouton, 2017, p. 124-151).

16 Entretien de Thomas Mirasol, président de la division en charge des ventes à l'international, accordé au Business Times (Singapour) en mai 2014 et repris aux Philippines par le quotidien The Philippine Star (20 juin 2014).

17 Fernando Lopez a notamment exercé un mandat de sénateur, il a été secrétaire d'État puis vice-président de la République des Philippines.

18 Introduction en bourse de la holding de tête. 
On note également le caractère assumé de cette substitution à l'État de la part du groupe Ayala, mais également la justification avancée : avoir de telles prérogatives permet au promoteur de produire des quartiers qui fonctionnent, dans lesquels les réseaux techniques sont intégrés dès la planification. En d'autres termes, la firme vient combler un vide laissé par une puissance publique défaillante, incapable de mener à bien un travail de planification urbaine cohérent et de le faire respecter, et guère plus à même d'assurer le quotidien des services publics qui permettent le fonctionnement des villes. L'histoire de la région métropolitaine de Manille en est une illustration. Si l'on observe les plans d'urbanisme successivement adoptés dans la région capitale ${ }^{19}$, on peut faire le constat d'une incapacité des institutions publiques à appliquer les décisions qui en découlent. Elles sont débordées par les acteurs privés. Plus précisément comme l'affirme Paulo Alcazaren (2013), si un facteur explicatif doit être mis en avant pour comprendre ce phénomène, ce doit être l'impossibilité des acteurs publics à accéder aux ressources foncières nécessaires. En effet, plus de dix master plans ont été dessinés et adoptés depuis le début du $\mathrm{XX}^{\mathrm{e}}$ siècle sans être mis en œuvre, et les autorités publiques qui ne sont pas propriétaires du foncier, n'ont jamais été en mesure d'acquérir les terrains stratégiques. Ces derniers, dès la publication des plans, prennent de la valeur et font l'objet de spéculation de la part d'acteurs privés. Ils sont ainsi rendu hors d'atteinte des autorités publiques, qui ne peuvent rivaliser financièrement.

Cette légitimation de l'action privée par les acteurs concernés est d'autant plus recevable que leurs réalisations tiennent la comparaison. Ainsi, un commentateur philippin insiste sur la qualité des espaces produits par ces acteurs privés, par opposition au reste du tissu urbain : "Imagine if the late Col. Joseph McMicking didn't start Ayala's Makati developments or if his successors didn't follow through as well as they did, we wouldn't have this patch of sanity and modernity today. Metro Manila will be one big sea of haphazard urbanization typical of dysfunctional Third World cities ${ }^{20}$.»

19 Les premiers plans ont principalement concerné la municipalité de Manille, puis la municipalité de Quezon City choisie comme capitale pour les Philippines de 1948 à 1976.

20 The Philippine Star, 20 juin 2014. Le quartier de Makati est le CBD le plus important du pays. 
La notion de " modernité » est de fait invoquée par les promoteurs immobiliers et reprise de manière presque systématique lorsque l'on évoque les grandes opérations de (re)développement localisées dans la région métropolitaine de Manille. Le discours n'est pas propre aux Philippines, comme l'ont montré Roy et Ong (2013) dans leur étude sur les grandes villes asiatiques avec l'expression de worlding cities. Les imaginaires convoqués par les promoteurs immobiliers sont ceux de Singapour ou de Dubaï, et les discours mettent l'accent sur le caractère "international " des espaces urbains. On notera à titre d'exemple que les transports collectifs populaires philippins (jeepneys, tricycles) sont bannis des CBD gérés par les groupes privés : seuls sont autorisés les taxis et les véhicules privés. La fiabilité des réseaux est également mise en avant, d'autant plus qu'elle est un facteur critique pour attirer des entreprises et notamment celles qui ont une activité liée au Business Process Outsourcing (BPO). Ce secteur qui regroupe les centres d'appel, les services de retranscription légale et médicale ou encore de comptabilité, requiert une continuité dans ses process de travail. Ainsi, une coupure d'électricité ou des services de télécommunication sont particulièrement dommageables, et les entreprises ont par exemple l'obligation contractuelle de s'implanter dans des zones qui présentent des garanties en matière d'approvisionnement en électricité ${ }^{1}$. Ainsi, les grands conglomérats apparaissent comme les seuls acteurs à même de pallier les déficiences de la puissance publique et affichent la volonté d'apparaître comme une force de la modernité, la seule capable d'emmener le pays sur la voie du développement.

\section{Conclusion}

Si la faiblesse de l'acteur public légitime aux yeux de la population la place qu'occupent les grands groupes philippins dans la production urbaine et la fourniture de services publics, ces derniers confortent leur position en développant une communication qui met en avant leur implication dans le récit national. Une telle implication n'est pas

21 Entretien réalisé avec un cadre de l'organisation IBPAP, qui représente les intérêts des entreprises du secteur du BPO, Taguig City, mai 2014. 
sans soulever des questions par rapport à la nature de l'espace urbain ainsi créé. L’exemple évoqué plus haut de New Makati, le quartier d'affaires historique de Manille, en est une illustration frappante. Dans son étude sur le développement de ce quartier, Marco Garrido (2013) montre que le projet porté par la famille Zobel de Ayala n'est pas uniquement motivé par le profit, et qu'il répond à une volonté de " moderniser " la capitale philippine, d'utiliser les outils de la planification urbaine pour créer un espace rationnel, qui servira de modèle pour la région capitale. Surtout, un tel projet n'est a priori pas synonyme d'exclusion des classes populaires :

"Ayala planned for a socioeconomically diverse community, with separate subdivisions built to accommodate lower-middle to high income residents; it enacted prohibitive building and land use restrictions (protested as excessive at the time); and took pains to regulate lot sizes, amenities, and the width of roads, not purely in order to profit - why such a surplus of consideration otherwise? - but also to create a different kind of social space » (Garrido, 2013, p. 170).

Cependant, ce projet moderniste se confond rapidement avec un autre projet, porté par les classes supérieures de l'espace urbain : celui d'une sécession, d'un isolement vis-à-vis des classes sociales qui leur sont inférieures. De fait, le quartier de Makati développé par Ayala Land, Inc. est caractérisé par une grande homogénéité sociale. La délégation " en bloc » de la production urbaine à l'échelle de quartiers, voire de villes entières, à des groupes privés pose de manière frontale la question des usages de l'espace urbain. Même si leurs projets se veulent ancrés dans un récit national, les forces de marché dans lesquelles ils s'inscrivent ne vont pas dans le sens d'un intérêt général et favorisent plutôt les intérêts particuliers des populations les plus aisées. Ces espaces relèvent ainsi des pratiques de cherry-picking décrites par S. Graham et S. Marvin (2001), mais contrairement à la thèse des deux auteurs, la cause n'est pas tant la libéralisation des secteurs des utilities que l'économie politique de la fabrique urbaine locale. C'est le fait d'acteurs nationaux, ou éventuellement régionaux, qui prennent en charge des missions qui ailleurs auraient été du ressort de l'État. 


\section{Références}

Alcazaren P. (2013), "Metro Manila's urban chaos. What now? ", Conférence au Lopez Digital Media Series - ADMU, Quezon City, Philippines.

Banfield E. C., Wilson J. Q., (1966), City Politics, New York, Vintage Books, Random House.

Bello W. (2005), The Anti-Development State: The Political Economy of Permanent Crisis in the Philippines, Londres, Zed Books, 360 p.

Bonnet F. (2010b), « Les machines politiques aux États-Unis. Clientélisme et immigration entre 1870 et 1950 ", Politix, vol. 23, n 92, p. 9-29.

Chevallier J.-J. (1966), Les grandes auvres politiques de Machiavel à nos jours, Paris, Armand Colin.

Defeuilley C. (2013), " Portrait d'entreprise. La New River Company ", Flux, n' 91 , p. 56-65.

Garrard J. (1992), "Le scandale du gaz à Salford en 1887 ", Flux, vol. 8, $\mathrm{n}^{\circ} 10$, p. 8-24.

Garrido M. (2013), «The Ideology of the Dual City: The Modernist Ethic in the Corporate Development of Makati City, Metro Manila: The modernist ethic in the dual city of Makati ", IJURR.

Graham S., Marvin S. (2001), Splintering Urbanism: Networked Infrastructures, Technological Mobilities and the Urban Condition, Londres, Routledge, $512 \mathrm{p}$.

Guéguen C. (2013), "Institutions, société civile et héritage de la Loi martiale dans l'aménagement de la baie de Manille (Metro-Manila, Philippines) ", Cybergeo.

Hill C. (1972), The World Turned Upside Down, Radical Ideas During the English Revolution, Londres, Penguin Books.

Hobbes T. (1983 [1651]), Léviathan, Paris, Sirey.

Hutchcoft P. (2008), "The Arroyo Imbroglio in the Philippines ", Journal of Democracy, vol. 19, n 1 , p. 141-155.

Hutchcroft P. (1991), "Oligarchs and Cronies in the Philippine State: The Politics of Patrimonial Plunder », World Politics, vol. 43, n 3, p. 414-450. 
Kang D. C. (2002), Crony Capitalism: Corruption and Development in South Korea and the Philippines, Cambridge, Cambridge University Press, $222 \mathrm{p}$.

Kishiue A. (2000), The Concept of establishing integrated community in Makati City by Ayala, mémoire de master, University of the Philippines School of Urban and Regional Planning.

Krinks P. (2003), The Economy of the Philippines: Elites, Inequalities and Economic Restructuring, Londres, Routledge, 423 p.

Lorrain D. (2000), "The construction of urban services models 2001 », dans Bagnasco A., Le Galès P. (éds), Cities in Europe, Cambridge, Cambridge University Press, p. 153-177.

Lorrain D. (2016), "Les conglomérats familiaux (4). Salim Group ", Flux, $\mathrm{n}^{\circ} 106$, p. 86-100.

Lorrain D., Mouton M. (2017) «Les conglomérats familiaux (5) : Ayala Corporation ", Flux, n 107, p. 91-103.

Lowi T. J. (1969), « Machines politiques ou bureaucraties administratives, le gouvernement des villes américaines ", Sociologie du travail, $\mathrm{n}^{\circ} 4$.

Magno-Ballesteros M. (2000), «The urban land and real estate market in Metro Manila: a socio-economic analysis ", mémoire de thèse, Radboud University Nijmegen.

Matheu M. (2002), "La régulation des services publics en réseaux, ou la lente émergence d'une innovation majeure ", Entreprises et histoire, vol. 30, n 3, p. 115-135.

McCoy A. W. (1993/2009), An Anarchy of Families: State and Family in the Philippines, Madison, University of Wisconsin Press, 590 p.

Mouton M. (2017), "Changement urbain sous tension. Service électrique et modernité dans le Grand Manille (Philipines) ", mémoire de thèse, Université Paris-Est, 320 p.

Neild R. (2002), Public Corruption. The Dark Side of Social Evolution, Londres, Anthem Press.

North D. C., Wallis J. J., Weingast B. R. (2010), Violence et ordres sociaux, Paris, Gallimard.

Roy A., Ong A. (2013), Worlding cities: Asian experiments and the art of being global, Chichester, Wiley Blackwell. 
Spitz J.-F. (1994 [1689]), «Introduction » à John Locke, Le second traité du gouvernement, Paris, Presses universitaires de France, p. V-LXXX.

Tarr J. A. (1989), "Infrastructure and City-Building in the Nineteenth and Twentieth Centuries ", dans Hays Samuel P. (éd.), City at the Point: essays on the social history of Pittsburgh, Pittsburgh (PA), University of Pittsburgh Press, p. 213-263.

Tiglao R. (2014), " How Salim Group skirted foreign ownership limits ", The Manila Times, 3 mars, http://www.manilatimes.net/how-salimgroup-skirted-foreign-ownership.

World Bank (1992), «Governance and development », rapport n 10650, $69 \mathrm{p}$. 


\title{
La firme d'infrastructures locale et le rééchelonnement
}

\author{
Daniel Florentin \\ Pauline Gabillet \\ Catalina Duque Gomez
}

\section{Les firmes locales au cœur de transformations}

Au cours de la dernière décennie, les firmes locales d'infrastructures ont fait l'objet d'un intérêt scientifique et politique renouvelé pour deux faisceaux de raisons.

Tout d'abord, elles ont été au centre de débats d'économie politique concernant leur statut juridique, ce qui s'est traduit par des travaux sur les processus de remunicipalisation des utilities, scandés par les récits de quelques cas paradigmatiques comme Berlin, Bogota ou Grenoble (Blanchet, 2014; Hall et al., 2013 ; Hüesker et al., 2011).

Elles ont également été au cœur de débats sociotechniques et urbains au cours des dernières années, car ces firmes locales ont été souvent dépeintes comme des leviers majeurs pour opérer des transitions énergétiques (Ambrosisus, 2012 ; Nieswandt, 2012).

Ces deux grands débats occultent ou négligent cependant une transformation importante de ces firmes, qui a émergé de la comparaison entre nos terrains d'étude, et qui tient à ce que nous appelons, en traduisant le terme rescaling, un rééchelonnement multiple, à la fois territorial, économique et régulatoire. C'est ce rééchelonnement, ses modalités et son impact sociotechnique qui constituent le cœur de ce chapitre et donnent à voir une transformation infrastructurelle importante. Il vient poursuivre de manière relativement différente des réflexions entamées dans la littérature anglo-saxonne, centrées 
principalement sur un réajustement et une transformation de l'acteur étatique (Brenner, 2004), là où notre objet est l'entreprise de services urbains.

La firme d'infrastructure est un point de départ. Elle n'est cependant pas un horizon borné, mais bien une porte d'entrée pour analyser des transformations qui la dépassent, et notamment les transformations urbaines, qui sont souvent laissées de côté par les études sur les transformations infrastructurelles (Monstadt, 2007 ; Monstadt, 2009). Nous cherchons ainsi à nous prémunir de l'écueil pointé par van Vliet et Shove, selon qui les études sur les changements dans les firmes d'infrastructures pèchent souvent par leur caractère monodimensionnel, se contentant soit d'en rester à une observation de la transformation institutionnelle (niveau macro) soit de se focaliser uniquement sur la " manipulation du comportement des ménages " (niveau micro) (van Vliet et al., 2005). Notre optique se situe donc à l'interface entre des questionnements gestionnaires, des enjeux techniques et des régulations sociopolitiques (notamment via une attention portée aux usages et aux usagers).

Nous sommes convaincus, avec Offner, qu'un réseau d'équipement interagit avec le territoire qu'il dessert (Offner, 2000), faisant du réseau un objet sociotechnique impliquant la ville, le gestionnaire, les usagers, et pouvant contraindre l'urbanisme (Bouleau, Richard-Ferraudji et Werey, 2011). Comme l'explique très clairement Sylvy Jaglin, "dédiés aux problèmes concrets de la desserte, les organismes en charge de leur gestion représentent un "lieu" stratégique d'observation de la production des règles et des principes d'action qui encadrent le changement réel dans les villes» (Jaglin, 2005, p. 22). La focale sur les entreprises permet en fait de parler autrement des questions de transformation urbaine, et notamment des échelles et des modalités de la maîtrise des réseaux (Martinand, 2001).

Les travaux sur les firmes d'infrastructures se sont jusqu'ici principalement portés sur les modèles nationaux d'organisation des services (Barraqué, 1995) ou les grands groupes multinationaux, travaillant des figures archétypales comme la "firme locale-globale " (Lorrain, 2005) et l'idée que la croissance de ces grands groupes serait le reflet de nouveaux "capitalismes urbains " (Lorrain, 2002). Notre approche vient compléter ce tableau, en ne nous focalisant pas sur 
les têtes de pont métropolitaines, mais en portant notre attention sur une figure plus ordinaire (Robinson, 2006), celle de la firme locale d'infrastructure.

\section{Trois firmes locales d'infrastructure, trois contextes sociopolitiques et sociotechniques différents}

Nous travaillons sur trois terrains fort différents, mais en nous centrant à chaque fois sur la firme locale d'infrastructures et sur ses évolutions. Ces trois entreprises peuvent être présentées très brièvement.

La première est située à Magdebourg, dans l'Est de l'Allemagne (capitale du Land de Saxe-Anhalt, à une centaine de kilomètres de Berlin). Les services techniques urbains sont principalement gérés par l'opérateur multiservices local, le Stadtwerk de Magdebourg (SWM), qui est un exemple classique du modèle municipal fort ${ }^{22}$ de services urbains (Krämer, 1993 ; Barraqué, 1995). Le Stadwerk est en fait inséré dans les dynamiques propres au contexte de transition post-socialiste (Golubchikov et al., 2014 ; Sykora et Bouzarovski, 2012), qui ont vu un bouleversement majeur à la fois des cadres organisationnels et des pratiques de consommation. L'opérateur a ainsi dû composer avec des diminutions très importantes des consommations dans les différents services urbains, aussi bien dans l'eau, le gaz que le chauffage urbain. Ces diminutions ont forcé la firme locale à initier une transformation massive de ses services d'eau et d'énergie.

Le second cas est situé à Grenoble, dont le système énergétique dévie du modèle français étatique très centralisé. Des décisions politiques du début du $\mathrm{XX}^{\mathrm{e}}$ siècle ont ainsi conduit la ville à s'appuyer sur une firme publique locale, Gaz Électricité de Grenoble (GEG), dépendant malgré tout de marchés du gaz et de l'électricité régulés au niveau national. Ce cadre de régulation est d'ailleurs remis fortement en question à la

\footnotetext{
Son capital est toutefois mixte, avec une majorité $(54 \%)$ détenue par la municipalité, le reste étant l'apanage de deux compagnies privées du monde de l'eau et de l'énergie, Gelsenwasser (qui est à l'origine un groupement d'entreprises publiques locales) et E.ON Avacon.
} 
fois par les processus de libéralisation touchant les marchés de l'énergie et par l'intégration des objectifs climatiques et énergétiques dans les programmes et politiques publiques.

Le dernier cas s'inscrit lui dans le contexte du marché fortement libéralisé de l'énergie en Colombie, dans la ville de Medellín, la seconde plus grande ville du pays. La firme locale d'infrastructures, Empresas Publicas de Medellín (EPM) a été, tout au long du XX $X^{\mathrm{e}}$ siècle, un acteur historique du développement de l'électricité non seulement à l'échelle de la ville, mais de la région. Elle a réussi à traverser les multiples réformes néolibérales des années 1990 qui ont déstructuré le modèle ancien des opérateurs multiréseaux municipaux, sans être privatisée ni démembrée.

Ces trois contextes sont certes variés aussi bien institutionnellement que géographiquement. Cependant, leur mise en perspective permet de voir émerger des trajectoires relativement similaires, et notamment dans les stratégies adoptées pour faire face à des marchés de l'énergie très concurrentiels. Ces convergences dépassent le stade de la simple coïncidence. Même si ces trois firmes ne représentent pas l'ensemble des firmes d'infrastructures, elles incarnent une tendance et une trajectoire suivie par de nombreuses firmes d'ampleur et de taille comparables. Cette trajectoire se traduit par la mise en place d'un nouveau régime infrastructurel (Monstadt, 2009), qui permet de lire les modifications des systèmes énergétiques à l'œuvre. Ce régime repose sur trois piliers, qui constituent les trois sections suivantes de cet article : le rééchelonnement de leur territoire de desserte, le rééchelonnement de leur modèle économique, le rééchelonnement de leur relation avec la ville. Ces trois transformations sont cruciales pour décrypter le contexte dans lequel les transformations énergétiques sont en train d'apparaître.

\section{Le rééchelonnement territorial des firmes locales d'infrastructures}

Dans ce processus relativement silencieux, mais décisif de transformation des firmes d'infrastructures locales, on peut ainsi observer une trame commune, celle d'une modification de la territorialité de ces firmes (Furlong, 2014). La libéralisation des marchés de l'énergie et le développement croissant de nouvelles formes de production et de 
distribution d'énergie ont contribué à créer de nouveaux arrangements spatiaux dans les systèmes énergétiques. Au cours des quinze dernières années, de nouvelles échelles de gouvernance sont ainsi apparues, qui ont contribué à produire une nouvelle géographie des opérateurs de services urbains.

Pareille transformation a pris la forme d'un rééchelonnement territorial, qui fait indirectement écho aux travaux de Neil Brenner et à l'hypothèse qu'il défend selon laquelle des processus de réagencement de l'État s'étaient traduits par de nouvelles formes de redistribution (spatiale) à l'échelle régionale, et qu'il a rassemblés sous le terme de "projets politiques néo-fordistes "(Brenner, 2004, p. 466). Le réagencement que connaissent les firmes locales d'infrastructures n'est bien entendu pas de la même ampleur que ce que décrit Brenner, mais il partage avec lui quelques caractéristiques. À travers les divers changements d'échelle de leurs territoires de desserte qu'elles ont mis en place, les firmes locales d'infrastructure ont changé d'ampleur, délaissant leur caractère uniquement local pour devenir toujours davantage des acteurs multi-échelles. Nos trois cas vont dans ce sens et révèlent un double mouvement d'expansion au niveau local en continuité avec les infrastructures existantes et d'extension au niveau régional, voire national.

Dans le cas de Magdebourg, ce réagencement territorial est très puissant, bien qu'il soit marqué par l'alternance de processus complexes de déterritorialisation et reterritorialisation. Les premières étapes de la libéralisation des marchés de l'énergie ont ainsi été l'occasion pour l'opérateur de développer des activités dans d'autres villes afin d'étendre son marché. C'est dans ce mouvement que le Stadtwerk de Magdebourg est devenu actif dans des villes du Nord del'Allemagne comme Hambourg ou Schwerin. Le processus a été poussé assez loin, provoquant même une forme de déterritorialisation de la firme locale, puisque l'entreprise s'est mise à vendre plus d'électricité en dehors de Magdebourg que dans son giron d'action traditionnel. Cette transformation a été rapidement considérée comme porteuse de trop grands risques par certains des directeurs de l'entreprise, ce qui a provoqué un changement de stratégie de la firme, qui désormais favorise l'échelle régionale dans ses politiques d'expansion, dans une logique de reterritorialisation. 
La zone d'influence de l'opérateur a ainsi été fortement étendue à l'échelle régionale, aussi bien dans les secteurs du gaz, de l'eau que de l'électricité. La firme a ainsi pris le contrôle technique de l'opérateur d'eau de la région de Schönebeck (WZV Schönebeck), au sud de Magdebourg, et a endossé la gestion commerciale du Stadwerk de Stendal. Des évolutions similaires se sont produites dans le secteur électrique : la firme locale de Magdebourg a ainsi pris des parts dans les opérateurs multiservices spécialisés dans l'énergie de Zerbst ou Stendal. L'ensemble de cette restructuration peut se lire comme une stratégie de consolidation et d'extension territoriale, un véritable upscaling $\mathrm{du}$ Stadtwerk. L'ambition qui se trouve derrière cette évolution est clairement définie par les dirigeants de l'entreprise : devenir une firme régionale et non plus simplement une firme locale/communale d'infrastructure.

Dans le cas de Magdebourg, tout comme d'ailleurs dans d'autres villes similaires comme Halle, l'opérateur de la métropole régionale étend progressivement son aire d'influence en prenant, depuis le début des années 2000, le contrôle des Stadtwerke locaux plus petits. Cela permet d'une certaine façon à la firme locale de compenser un marché historique déclinant ou sans croissance, dans ce que Tim Moss a nommé des " cold spots " (Moss, 2008) des réseaux, à savoir des territoires où la demande a chu de manière intense et où le niveau de service s'est fortement dégradé. Cette stratégie de croissance régionale permet à la firme locale de conserver une certaine viabilité économique, puisque près de $40 \%$ de ses bénéfices en 2013 venaient des entreprises ainsi intégrées dans un groupe plus large. D'une certaine manière, cette stratégie est la réplique presque à l'identique du schéma adopté par l'un des actionnaires du Stadtwerk de Magdebourg, Gelsenwasser, qui avait choisi d'étendre fortement sa zone d'influence dans le domaine de l'eau dans de nouvelles régions pour faire face à une demande déclinante dans la Ruhr qui était son territoire historique. Les opérateurs multiservices comme le Stadtwerk de Magdebourg deviennent ainsi progressivement des acteurs politiques régionaux et, de ce fait, des acteurs indispensables et inévitables des politiques de transitions ou d'adaptations énergétiques.

La firme locale d'infrastructures de Medellín a suivi, depuis les années 1990, une trajectoire similaire, même si la dialectique déterritorialisation/ reterritorialisation s'y exprime de façon assez différente. La firme a connu 
une métamorphose spatiale impressionnante, qui l'a fait largement sortir du cadre de son territoire d'origine. La stratégie d'expansion de la firme a été ainsi à la fois incrémentale et monumentale. Le réseau électrique a d'abord été étendu au niveau local, dans la vallée d'Aburra, qui constitue désormais la région métropolitaine de Medellín, et au niveau du département d'Antioquia. L'objectif premier de cette transformation était d'augmenter les capacités de production de la firme, afin de satisfaire une demande en énergie croissante (à l'opposé de ce que l'on observe à Magdebourg donc). À travers ces extensions successives, EPM a ainsi construit un réseau électrique très fortement intégré, qui représente en $201524 \%$ de la capacité de production nationale d'électricité. Cette stratégie d'expansion a permis à la firme locale de devenir non seulement un acteur local de l'arène énergétique, mais un acteur national. Durant la crise énergétique qui a touché le pays au début des années 1990, EPM est apparu comme un acteur clé au niveau national, en prenant en charge la construction d'un réseau électrique interconnecté et en mettant en place un nouveau plan augmentant la capacité d'électricité produite au niveau national (López Díez, 2003 ; Varela Barrios, 2010).

Par ailleurs, les réformes de libéralisation des services urbains ont forcé EPM à transformer sa structure administrative et à créer une holding appelée Grupo EPM. À travers ses différentes filiales, la holding a permis à EPM de continuer son changement d'échelle territoriale, de sorte que la firme locale est désormais présente dans sept régions en Colombie et dans six pays d'Amérique latine ${ }^{23}$. Ces multiples métamorphoses font qu'EPM n'est plus une petite firme locale, mais un groupe impressionnant tenu par la municipalité de Medellín, et dont les tentacules spatiales ont radicalement transformé la géographie des firmes locales. EPM a explicitement suivi une stratégie de déterritorialisation de ses activités : l'un des objectifs de Grupo EPM est en effet que $40 \%$ de son activité soit effectuée par l'entremise de ses filiales à l'international.

23 Grupo EPM est une holding constituée, au niveau national, de 6 filiales dans le secteur de l'eau et quatre dans le secteur de l'énergie, opérant en dehors de la zone d'Antioquia. Au niveau international, EPM est à la tête de 23 filiales, principalement dans le domaine de l'énergie, qui développent leurs activités au Mexique, au Chili, au Panama, au Costa Rica, au Guatemala et au Salvador. 
Cependant, cette forme de déterritorialisation ne signifie pas pour autant qu'EPM a abandonné la sphère locale : son modèle spatial a été bouleversé, mais la firme reste possédée localement et profondément ancrée dans son territoire régional historique.

Dans le cas de Grenoble, les possibilités d'un rééchelonnement de la firme sont plus réduites, en raison notamment du fait que la distribution d'électricité demeure un monopole national et bloque ainsi de nombreuses possibilités d'extension. Cependant, la firme locale a adopté de nouvelles formes de territorialisation (Jaglin, 2005) et cherche à étendre son cadre d'action originel. L'entreprise a ainsi développé de nouvelles installations d'énergies renouvelables en dehors de la ville et tend à favoriser de plus en plus l'échelle régionale pour développer ses divers projets. Les processus de déterritorialisation ne sont pas pertinents dans le cas grenoblois, où la firme locale cherche davantage à renforcer et à développer ses activités en s'appuyant sur son ancrage territorial historique en déployant de nouvelles installations et activités en cercles concentriques dont le cœur reste la ville de Grenoble. La firme a également développé de nouveaux produits qu'elle commercialise dans la région, ce qui nous conduit au deuxième volet de ce triple rééchelonnement.

\section{Le rééchelonnement économique : la diversification sectorielle de la firme locale d'infrastructures}

Le changement d'échelle territorial qu'ont connu et connaissent les firmes locales produit de nouvelles géographies de la gestion des infrastructures. Toutefois, cela n'épuise pas la compréhension du changement de régime infrastructurel qui est à l'œuvre. Cette mutation spatiale s'accompagne en effet d'une transformation du modèle économique de ces firmes, en l'occurrence d'une diversification des activités et d'une transformation des modalités permettant de produire de la valeur. Une tendance émerge de la confrontation de nos terrains et de certaines observations : les firmes locales d'infrastructures ne se limitent plus au simple transport ou à la distribution d'un fluide à travers un tuyau. Elles sont devenues des fournisseurs de services urbains beaucoup plus larges, qui dépassent largement leur mission traditionnelle. 
Un point important est de bien voir que cette diversification des activités est le corollaire direct des processus de rééchelonnement spatial : les deux processus convergent pour ancrer la firme dans un territoire plus vaste. Ce changement de modèle contribue à donner aux firmes un nouveau rôle dans l'arène urbaine, qui dépasse peut-être le simple cadre de la gestion des infrastructures.

Dans nos trois cas, la transformation du modèle économique traditionnel suit deux directions principales : une extension des services liés à la fourniture d'énergie et le développement de services qui ne sont pas liés au cœur de la mission d'un opérateur.

Le processus est particulièrement notoire dans le cadre du cas de EPM à Medellín. À travers ses différentes filiales, Grupo EPM a développé tout un panel d'activités et de services, qui inclut notamment la commercialisation d'appareils électroménagers (notamment au Salvador). Au niveau local, EPM, comme de nombreuses autres entreprises similaires, a également élargi son portefeuille de services, en l'orientant notamment plus fortement vers les usagers finaux, et en développant des services allant du conseil pour réduire les consommations à l'élaboration de programmes de crédits pour acheter des appareils électroménagers. Le processus est allé jusqu’à la création d'une carte de crédit EPM.

Toutefois, la partie la plus décisive de cette stratégie de diversification ne s'est pas jouée dans le domaine des services urbains, mais à travers des programmes de Responsabilité Sociale des Entreprises (RSE). La plupart de ces programmes ont été mis en place à travers une fondation dédiée, la Fundacion EPM. Par ce biais, la firme locale d'infrastructures se retrouve impliquée dans la gestion d'espaces publics, dans l'équipement, la construction et la gestion de bâtiments culturels ou éducatifs. On peut désormais emprunter un livre dans la bibliothèque EPM, aller se promener dans le Parque de los pies descalzos géré par EPM ou dans les Unidades de Vida Articulada construites par EPM. Cet engagement de la firme la fait participer à la fabrique urbaine, à la transformation physique de la ville tout comme à sa gestion. Par ce type de stratégie, la firme renforce sa visibilité au sein de la ville de Medellín, qui pourrait presque être renommée en EPMville. Cette politique d'image permet à la firme d'être à la fois plus visible et plus proche de ses habitants/usagers, et vise à renforcer l'attachement de ces usagers à 
l'entreprise. Elle contribue à un enracinement dans le contexte local, qui sert de compensation à la stratégie de rééchelonnement territorial de sa gestion. Au niveau international, EPM exporte également une image de Medellín, comme si la firme avait absorbé la ville. La diversification économique est ici mise au service d'un processus de territorialisation, qui permet à la firme de se légitimer comme un acteur majeur de la gouvernance locale.

Si l'on peut dire que Medellín semble se convertir en EPMville, Magdebourg, de la même façon, tend de plus en plus à devenir SWMville, en raison notamment de la politique de diversification menée par la firme. Par-delà l'offre renouvelée et étendue de services liés à l'énergie qui caractérise le tournant commercial ayant marqué les mondes de l'énergie, le Stadtwerk de Magdebourg a également diversifié ses missions en développant un nouvel outil, la carte SWM. Cette carte, gratuite, n'offre aucun service en lien avec les services techniques urbains. Pourtant, cet outil permet à ses usagers de bénéficier d'avantages commerciaux divers, comme des réductions dans certaines boutiques ou un accès libre à certains événements culturels. La firme elle-même apporte son soutien à certains événements culturels. Par ce biais, SWM envahit progressivement le quotidien des habitants de Magdebourg, non seulement par les innombrables voitures de techniciens qui parcourent la ville, mais également par les offres variées qui sortent du spectre classique des relations entre usager énergétique et fournisseur d'énergie. Cette stratégie d'ensemble, comme pour Medellín, participe d'un renforcement de la connexion entre la firme et son territoire.

Cette diversification est un peu plus limitée dans le cas de Grenoble, dans la mesure où la firme reste concentrée sur le cœur de métier historique, les questions énergétiques. Entre autres projets, la firme investit dans la production d'énergie renouvelable et dans la mise en place d'outils de smart grids. La transformation des activités est, dans ce contexte, principalement conditionnée par les opportunités financières liées aux politiques de transition énergétique élaborées au niveau national. 


\section{Le rééchelonnement régulatoire : les nouveaux arrangements entre ville et firme locale}

Le mouvement de transformation territoriale et de diversification économique des firmes locales d'infrastructures contribue en fait à un troisième rééchelonnement, qui voit les arrangements politiques entre la ville et la firme être profondément reconfigurés. Les transformations des firmes locales font émerger une tension grandissante entre d'un côté la volonté éminemment politique des dirigeants des firmes locales d'infrastructures de conserver une forme d'autonomie et, de l'autre, l'inclusion de ces mêmes firmes dans le paysage politique local. Les firmes locales d'infrastructures, comme firmes de services publics, sont à ce titre intriquées avec les autorités municipales. Leurs transformations, combinées avec un intérêt renouvelé des collectivités locales pour les questions énergétiques expliquent les nouvelles modalités de régulations (et de conflits) qui se mettent en place entre les pouvoirs locaux et les firmes locales d'infrastructures.

Dans les trois cas que nous avons pu confronter, la firme a été longtemps perçue par les autorités municipales comme une simple source de revenus.

À Grenoble, la firme bénéficie certes d'une certaine autonomie pour élaborer sa stratégie, mais son fonctionnement nourrit dans le même temps de façon substantielle les revenus de la ville. Pendant longtemps, la ville s'est cantonnée au rôle de validation des choix stratégiques d'expansion territoriale et de diversification économique, dans l'idée que cela assurait une pérennité aux revenus de la ville, sans se soucier particulièrement des dimensions proprement énergétiques et techniques de la firme.

Cependant, la mise en place de nouvelles politiques publiques sur les questions énergétiques et climatiques a profondément changé la donne. Les acteurs politiques locaux ont saisi cette opportunité pour reconsidérer le rôle de GEG, et en faire un outil de ces nouvelles politiques énergétiques urbaines (Gabillet, 2014). Au sein de l'administration de la ville, les départements en charge des questions de développement durable, d'environnement et d'urbanisme sont les grands défenseurs de cette vision, qui a abouti à l'élaboration des plans climats. Dans ce contexte, GEG est encouragée à participer à des projets urbains comme 
le développement d'écoquartiers ou des expérimentations de smart grids. Même si ces changements demeurent encore assez marginaux, ils attestent d'une volonté grandissante d'intégrer la firme locale d'infrastructure dans les politiques urbaines. Ce changement du rôle dévolu à la firme lui ouvre de nouvelles opportunités, et lui permet de faire reconnaitre son statut urbain et politique. Cette modification des relations entre ville et firme amène à des frictions en interne, au sein de la collectivité locale, mais également entre la ville et l'entreprise locale. La majeure partie de ces conflits a pour origine la volonté de la firme locale de préserver une forme d'autonomie industrielle et stratégique, dans un contexte marqué à l'inverse par une politisation croissante des enjeux énergétiques locaux.

Des frictions similaires peuvent être observées à Magdebourg entre la ville et la firme, en raison des transformations multiples de la firme. Une part importante des bénéfices du Stadtwerk de Magdebourg est désormais accumulée en dehors de la ville de Magdebourg, mais $60 \%$ de ces bénéfices sont transférés au budget de la ville, en vertu du nombre d'actions qu'elle possède. Cet état de fait de bénéfices extérieurs alimentant le budget d'une autre collectivité illustre les relations potentiellement complexes entre Magdebourg et sa région et le dilemme constant auquel doit faire face la firme locale d'infrastructure, oscillant entre sa mission communale historique, le soutien au développement local et ses nouvelles stratégies économiques d'expansion et de diversification.

La reconfiguration du rôle et de la place de la firme dans le paysage local est aussi à l'origine de nouvelles formes de conflictualités dans le cas de Medellín. Cela est particulièrement saillant dans certaines zones de la ville, lorsque les techniciens de la ville sont en désaccord manifeste avec les techniciens d'EPM. Dans cette lutte entre deux légitimités techniques, la ville, malgré sa volonté d'exercer un contrôle plus étroit sur la firme, lui reste souvent inférieure, et le pouvoir économique, technique et symbolique de la firme participe généralement d'une dépossession des techniciens de la ville au profit de ceux de la firme, ce qui ne va pas sans frictions. 


\section{Conclusion}

Notre approche des infrastructures se veut sociotechnique et centrée sur un acteur spécifique et souvent sous-étudié, la firme locale d'infrastructure. Son rôle est généralement largement sous-estimé dans les mutations urbaines à l'œuvre et l'émergence d'un nouveau régime infrastructurel et urbain.

En confrontant les évolutions d'un même type d'acteur dans trois contextes différents, nous avons pu voir émerger la trame commune d'une métamorphose à la fois discrète et décisive pour comprendre les réagencements des mondes urbains et énergétiques en cours. Cette évolution consiste en un déploiement sur de plus vastes territoires, une diversification des services offerts et une plus forte intrication entre l'offre de services et les politiques publiques locales. Le déchiffrement des modalités de cette transformation est essentiel pour analyser l'évolution des systèmes énergétiques urbains et les enjeux sociopolitiques qu'elle appelle.

Les transformations que nous avons pu identifier montrent également un point important : restreindre l'analyse des firmes d'infrastructures à des enjeux techniques et à des mondes uniquement centrés sur le tuyau et le service serait un contresens urbain. Les firmes jouent en effet de nouveaux rôles qui en font un acteur décisif du développement urbain, une quasi-structure de la fabrique urbaine et pas uniquement une firme d'infrastructure.

\section{Références}

Ambrosius G. (2012), "Geschichte der Stadtwerke », dans Bräunig D., Gottschalk W., Stadtwerke. Grundlagen, Rahmenbedingungen, Führung und Betrieb, Baden-Baden, Nomos, Schriftenreihe Öffentliche Dienstleistungen, $n^{\circ}$ 56, p. 35-52.

Barraque B. (1995), "Les politiques de l'eau en Europe », Revue française de science politique, vol. $45, \mathrm{n}^{\circ} 3$, p. 420-453.

Blanchet T. (2014), «Une privatisation partielle sous contrainte. Le cas de la Compagnie des eaux de Berlin ", Actes de la recherche en sciences sociales, $n^{\circ} 203$, p. $60-73$. 
Bouleau G., Richard-Ferroudji A., Werey C. (2011), " Patrimoines à réapprécier ", dans Bouleau G., Guérin-Schneider L. (éds), Des tuyaux et des hommes, Versailles, Quae, coll. «Indisciplines », p. 49-66.

Brenner N. (2004), "Urban governance and the production of new state spaces in western Europe, 1960-2000 ", Review of International Political Economy, vol. 11, n 3, p. 447-488.

Furlong K. (2014), "Water and the entrepreneurial city: The territorial expansion of public utility companies from Colombia and the Netherlands ", Geoforum, n ${ }^{\circ}$ 13, http://dx.doi.org/10.1016/j. geoforum.2014.09.008 (consulté le 17 juillet 2017).

Gabillet P. (2014), "Energy networks in an urban area, a coordination under construction ", paper presented at the conference "Interlinking urban infrastructure systems: from sectoral to integrated approaches ", Tützing, $20 \mathrm{p}$.

Golubichikov O., Badyina A., Makhrova A. (2014), "The Hybrid Spatialities of Transition: Capitalism, Legacy and Uneven Urban Economic Restructuring ", Urban Studies, vol. 51, p. 1-17.

Hall D., Lobina E., Terhorst P. (2013), "Re-municipalisation in the early 21st century: water in France and energy in Germany ", International Review of Applied Economics, vol. 27, n 2, p. 193-214.

Hüesker F., Moss T., Naumann M. (2011), «Managing Water Infrastructures in the Berlin-Brandenburg Region between Climate Change, Economic Restructuring and Commercialisation ", Die Erde, n 142, p. 187-208.

Jaglin S. (2005), Services d'eau en Afrique subsaharienne. La fragmentation urbaine en question, Paris, CNRS Éditions, 244 p.

Kramer A. (1993), "Querverbund - la gestion transversale des services publics en Allemagne ", dans Barraqué B. (éd.), La ville et le génie de l'environnement, Paris, Presses de l'École des Ponts et Chaussées, p. 197204.

Lopez Diez J.-C. (2003), El agua que nos cae. Gestión de los sistemas hidricoeléctricos: tensions entre lo público y lo privado (1890-1980), Medellín, Fondo Editorial Universidad Eafit.

Lorrain D. (2002), "Capitalismes urbains : la montée des firmes d'infrastructures ", Entreprises et histoire, vol. 3, n 30, p. 7-31. 
Lorrain D. (2005), «Urban Capitalisms: European Models in Competition ", International Journal of Urban and Regional Research, vol. 29, $\mathrm{n}^{\circ} 2$, p. 231-267.

Martinand C. (2001), La maîtrise des services publics urbains organisés en réseaux, Paris, Conseil économique et social, $83 \mathrm{p}$.

Monstadt J. (2009), "Conceptualizing the political ecology of urban infrastructures: insights from technology and urban studies ", Environment and Planning, vol. 41, p. 1924-1942.

Monstadt J. (2007), "Urban governance and the transition of energy systems: institutional change and shifting energy and climate policies in Berlin ", International Journal of Urban and Regional Research, vol. 31, p. 326-343.

Moss T. (2008), « "Cold spots” of urban infrastructure: shrinking processes in Eastern Germany and the Modern Infrastructural Ideal », International Journal of Urban and Regional Research, p. 436-451.

Nieswandt F. (2012), "Der Querverbund im Kontext kommunalen Wirtschaftens ", dans Bräunig D., Gottschalk W., Stadtwerke. Grundlagen, Rahmenbedingungen, Führung und Betrieb, Baden-Baden, Nomos, Schriftenreihe Öffentliche Dienstleistungen, $n^{\circ}$ 56, p. 181-198.

Offner, J.-M. (2000), " "Territorial deregulation”: local authorities at risk from technical networks ", International Journal of Urban and Regional Research, vol. 24, n 1, p. 165-182.

Robinson J. (2006), Ordinary Cities: Between Modernity and Development, Londres, Routledge, 204 p.

Shove E., Walker G. (2007), «Caution! Transitions ahead: politics, practice, and sustainable transition management ", Environment and Planning, $\mathrm{n}^{\circ}$ 9, p. 763-770.

Sykoral., Bouzarovki S. (2012), «Multiple transformations: conceptualising the postcommunist urban transition ", Urban Studies, vol. 49, $\mathrm{n}^{\circ} 1$, p. 43-60.

Varela Barios (2010), "Estrategias de expansión y modos de gestión en Empresas Públicas de Medellín, EPM», Estudios Políticos, n 36, p. 141-165.

(van) Vliet B., Chappels H., Shove E. (2005), Infrastructures of consumption. Environmental Innovation in the Utility Industries, Londres, Earthscan, Routldege, $130 \mathrm{p}$. 

LA VILLE POST-RÉSEAU 



\title{
Au-delà de la ville des réseaux
}

\author{
Olivier Coutard \\ Jonathan Rutherford
}

\section{La ville des réseaux hégémonique}

Pendant la période historique qui s'étend approximativement du milieu du XIX ${ }^{\mathrm{e}}$ siècle au début du XXI ${ }^{\mathrm{e}}$, dans les pays du Nord, le développement de systèmes d'infrastructures en réseaux a été en même temps un moteur, un instrument et une conséquence de l'avènement de nouvelles formes de relations dans les territoires, dans les organisations et dans les pratiques sociales, à toutes les échelles spatiales et entre celles-ci. Il en est résulté la fourniture de services, et un accès à ceux-ci, plus ou moins homogène sur un plan social et spatial. Ces services ont été fournis à une échelle parfois locale, parfois plus large, par un vaste système technologique à planification et à gestion centralisées, dans le cadre d'une concession exclusive sur un territoire donné et qui se trouve uni, à son tour, par ce système (voir, par exemple, Tarr, 1979; Hughes, 1983 ; Tarr et Dupuy, 1988 ; Dupuy, 1991 ; Offner, 2000).

Le développement généralisé d'infrastructures en réseaux, notamment en Europe de l'Ouest, en Amérique du Nord et au Japon, est allé de pair avec l'essor d'une idéologie des réseaux qui peut être résumée à trois propositions essentielles : le réseau est le moyen le plus efficace de fournir les services publics urbains ; la performance d'un réseau est proportionnelle à sa taille (portée spatiale, nombre et diversité d'utilisateurs connectés) ; la solution aux problèmes soulevés par les réseaux réside dans les réseaux eux-mêmes (dans leur expansion, dans une plus grande centralisation de leur gestion et dans l'accroissement de leur sophistication technique) (Coutard, 2010). Le développement matériel des réseaux dans les pays du Nord et l'idéologie des réseaux se sont renforcés l'un l'autre, et les deux phénomènes réunis ont été le moteur 
de la diffusion mondiale de la "ville des réseaux " comme modalité sociotechnique hégémonique étayant des formes de territorialité urbaine indissociablement aérolaires et réticulaires.

Au Nord, où s'est accompli de manière universelle "l'idéal infrastructurel moderne " (Graham et Marvin, 2001), l'avènement, au sens historique, de la ville des réseaux, à partir du milieu du XIX ${ }^{\mathrm{e}}$ siècle, est apparu comme étant lié à différentes formes d'intégration ou de solidarité :

- intégration socio-économique par le biais de la fourniture de services essentiels homogènes sur l'ensemble des espaces urbanisés, cette fourniture bénéficiant de subventions croisées ;

- intégration fonctionnelle, car les infrastructures en réseaux ont facilité et étayé des pratiques spatiales à l'échelle d'espaces de peuplement de plus en plus étendus ;

- intégration politique, grâce à la coopération entre des gouvernements locaux adjacents, encouragée par la volonté de fournir des services homogènes à des régions urbaines entières ;

- "intégration métabolique ", car le développement de systèmes d'infrastructures en réseaux vastes, monopolistiques et à gestion centralisée a eu pour résultat, et aussi pour base d'appui, l'appropriation de ressources (éventuellement distantes) au bénéfice du développement de l'ensemble de la région urbaine.

Il est cependant essentiel de signaler que les " villes des réseaux " se sont présentées et se présentent encore sous des formes très diverses et que la diffusion universelle des systèmes d'infrastructures en réseaux a facilité et a été facilitée en retour par les différents types, les différents degrés et les différentes combinaisons d'agglomération/concentration et d'expansion/dispersion qui ont marqué le développement urbain contemporain dans les pays du Nord (Soja, 2000 ; Castells, 2010 ; Brenner, 2013). Autrement dit, les configurations sociospatiales des infrastructures ne déterminent pas automatiquement des modèles urbains, mais elles y sont étroitement associées.

Dans les pays du Sud, ce sont des configurations infrastructurelles très diverses qui ont prévalu, de manière différenciée avec les pays du Nord dans lesquels cette image idéale de la ville des réseaux a été plus 
ou moins mise en œuvre aux plans matériel, territorial et politique. Le modèle de la ville des réseaux a été activement mis en place dans certains cas (par exemple en Amérique latine), quoiqu'avec des buts parfois ambivalents, tandis que dans d'autres il est resté un objectif très vague ou un slogan (voir les contributions à Coutard, 2008 ; Lorrain, 2011). À Santiago, par exemple, le raccordement de toutes les habitations aux réseaux essentiels, notamment depuis les années 1980, a facilité et même légitimé une politique résolue de ségrégation spatiale et de fragmentation fonctionnelle, sociale, fiscale et politique au niveau des autorités locales au sein de la région urbaine, et a également stimulé des processus d'étalement urbain (Pflieger et Matthieussent, 2008). À Buenos Aires, une politique strictement conforme à l'idéal infrastructurel moderne s'est traduite par la création d'un espace doté de réseaux de première qualité (dans le secteur central de la capitale fédérale) délimité par le périmètre des principales conduites d'eau, mais excluant la moitié de la population de la région urbaine (Botton et De Gouvello, 2008).

Ainsi donc, les recherches empiriques révèlent que des réseaux d'infrastructures plus ou moins omniprésents ont coexisté, et ont même été en corrélation, avec des disparités sociospatiales plus ou moins graves. Fondamentalement, cela traduit le fait que des infrastructures en réseau ne peuvent être mises en place, et ne peuvent produire d'effets sociospatiaux principalement intégrateurs, qu'en présence de conditions socio-économiques, institutionnelles et politiques plus larges, comprenant notamment un accroissement des revenus et de la richesse au fil du temps, l'existence à un certain degré d'un "État providence " ou d'une redistribution socialement progressive des revenus, la suppression de l'extrême pauvreté et un certain contrôle sur l'urbanisation et sur les processus de peuplement des villes. Cependant, même là où il n'a pas été accompli matériellement (dans la plupart des contextes dans les pays du Sud), l'idéal de la ville des réseaux s'est avéré politiquement et idéologiquement puissant. Les politiques promues par les organismes de financement internationaux et par les gouvernements nationaux et locaux s'en sont d'ailleurs largement inspirées et y ont puisé leur orientation. 


\section{La ville des réseaux contestée}

Dans des études récentes sur les infrastructures des villes du Sud, surtout sur l'eau et l'assainissement, des chercheurs ont exploré les implications urbaines importantes de l'absence ou de dysfonctionnements des grands systèmes techniques assurant l'accès aux services essentiels. Ils ont également étudié des alternatives à ces systèmes existant de longue date. Ils ont souligné le fait que l'idéal infrastructurel moderne a rarement été réalisé dans les villes du Sud (Swyngedouw, 2004 ; Jaglin, 2005 ; Coutard, 2008 ; Gandy, 2008). Ils ont aussi noté le caractère problématique de la transposition de ce concept venu du Nord dans des contextes urbains très divers et fragmentés en Afrique, en Asie et en Amérique latine (Bakker, 2003 ; Simone, 2004 ; McFarlane, 2008). En même temps, des travaux dans les domaines des changements environnementaux urbains et de l'écologie politique urbaine ont fait apparaittre les infrastructures comme l'un des principaux points de controverse quant à l'accès aux aménités environnementales et aux flux de ressources (Melosi, 2000 ; Guy et al., 2001). La production des infrastructures se révèle comme un processus politique urbain majeur de transformation environnementale inégalitaire qui estompe totalement les distinctions, de fait artificielles, entre la nature et la ville (Kaika et Swyngedouw, 2000 ; Gandy, 2002 ; Keil, 2003 ; Heynen et al., 2006 ; McFarlane et Rutherford, 2008).

Ces études montrent largement que dans toutes les régions du monde, quoique pour des raisons différentes selon les contextes nationaux, régionaux et locaux, les systèmes d'infrastructures en réseaux classiques sont de plus en plus mis en question comme forme hégémonique de fourniture des services essentiels. Ce changement est en train d'avoir des conséquences concrètes dans les politiques infrastructurelles, et ce à l'échelle mondiale. Là où les réseaux classiques étaient devenus historiquement hégémoniques, on favorise désormais le développement de configurations systémiques diverses. Là où les systèmes en réseaux n'ont pas été (entièrement) mis en place, on assiste au maintien durable d'autres configurations préexistantes.

Il faut cependant souligner que les transformations infrastructurelles ne prennent jamais la forme d'une transition massive et directe d'un grand système technique à un autre. Les systèmes existants ne sont pas 
démantelés et retirés pour être remplacés par des systèmes alternatifs flambant neufs considérés comme des remèdes technologiques absolus. Des modifications incrémentales, des ajustements ou des réparations à petite échelle, l'intégration de " technologies de médiation " (Furlong, 2011) ou de nouvelles couches de dispositifs apportant plus de flexibilité et plus de retour d'information aux systèmes d'infrastructures existants (qui comportent déjà plusieurs couches), peuvent aboutir à de petites ou de grandes reconfigurations dans le fonctionnement et l'expérience urbains (voir, par exemple, Moss, 2000). Cela s'applique également à la remise sur pied de systèmes technologiques ayant auparavant existé, qu'ils aient disparu ou aient été temporairement abandonnés, et qui réémergent, car leurs qualités ou leurs composants paraissent retrouver leur utilité (Shove, 2012).

Il est également évident que ces formes et ces degrés de changement technologique très différenciés s'articulent étroitement, de diverses manières (et au point d'en être indissociables), avec les rapports et les processus sociaux évolutifs, de telle sorte que les infrastructures doivent être considérées comme composées de co-configurations de divers " hardwares " (matériels) et " softwares " (logiciels) (Walker et Cass, 2007). Comme le prouvent les travaux anciens et actuels en matière de STS et de transitions, le changement sociotechnique est en fait un mélange contingent de différents types et degrés de changement, lié à la dynamique évolutive de l'innovation et de composants plus stables, que sont l'inertie, la dépendance au sentier et la matérialité " obstinée " des infrastructures (Bijker et al., 1987 ; Hommels, 2005 ; Geels et Schot, 2007).

Les changements dans les systèmes d'infrastructures ne se produisent donc jamais d'un seul coup ; ils donnent toujours lieu à des frictions et des tensions entre des dynamiques largement indépendantes. Cependant, certaines caractéristiques générales peuvent être identifiées dans les transformations en cours des infrastructures matérielles et/ou de l'idéal infrastructurel :

- un passage de l'homogénéité (effective ou recherchée) à la diversité (reconnue) - et à la sélectivité ciblée - des espaces infrastructurels, y compris sous des formes diverses de recomposition des services, des pratiques, des technologies, des normes, des flux, des fournisseurs de services, etc. ; 
- des reconfigurations et des changements d'échelle au sein des " espaces de solidarité " liés à ces systèmes, les solidarités à grande échelle se réarticulant avec des formes renouvelées d'« autonomie » locale ;

- une solidarité sociospatiale de plus en plus fondée sur la division (impliquant l'appropriation) des ressources, qui se combine avec les solidarités classiques fondées sur la fourniture de services homogènes partagés ;

- une évolution, en matière de services, de formes collectives de pratiques, de normes et d'attentes vers des formes plus individualisées ou plus diversifiées ; et en rapport avec celui-ci, un passage d'infrastructures "à taille unique " à des infrastructures personnalisées, "sur mesure ";

- l'apparition de nouvelles formes d'appropriation individuelle ou collective des infrastructures et de nouvelles significations sociales et politiques des infrastructures ;

- la circulation internationale et interurbaine de modèles favorisant ces changements.

\section{Infrastructures effervescentes}

Les infrastructures deviennent ainsi de plus en plus hétérogènes quant aux éléments et aux significations qui les composent, quant à leurs topologies, leurs échelles et leurs frontières, et quant à la multiplicité des manières dont elles sont abordées et "vécues ». Les flux, mailles et composants des systèmes de gestion des eaux, des énergies, des déchets et autres subissent actuellement des formes multiples d'expansion, de différenciation, de rénovation et des remodelage vers des configurations diverses et contingentes qui redéfinissent les modalités d'inscription des infrastructures dans les tissus urbains et la notion même d'infrastructure (Coutard et Rutherford, 2016). De nouvelles écologies infrastructurelles émergent, en même temps que les flux de ressources et les techniques de contrôle et de gestion subissent des reconfigurations " locales" particulières qui recomposent les frontières, les valeurs et le fonctionnement des systèmes technologiques. Les infrastructures sont constamment négociées, produites et reproduites par les techniciens et les utilisateurs dans leur vie et leur travail quotidiens, et sont ainsi 
façonnées pour et par des appropriations, des expériences et des pratiques individuelles et collectives. Suivre et cartographier cette sphère vibrante et effervescente exige l'extension et le renouvellement incessants des perspectives, des méthodes et des outils analytiques.

Soulignons enfin, pour conclure, que les significations sociopolitiques des transitions infrastructurelles urbaines en cours sont par essence ambivalentes. De même que l'intégration effective dans et à travers la " ville des réseaux " a toujours dépendu de conditions sociopolitiques plus larges, aucun effet urbain des configurations sociotechniques " alternatives " n'est automatique : ces effets sont toujours façonnés par les caractéristiques particulières des sociétés urbaines dans lesquelles les infrastructures s'inscrivent et qu'elles façonnent à leur tour. Les configurations infrastructurelles " décentralisées " ou distribuées, émergentes ou existantes, n'apportent pas par elles-mêmes une plus grande durabilité, environnementale ou autre, car elles peuvent s'avérer au moins aussi gourmandes de ressources que les grands systèmes techniques centralisés. Les nouveaux aménagements technologiques pourraient très bien perpétuer le contrôle centralisé, et/ou être façonnés par et pour des intérêts sociaux dominants existants et ainsi reproduire des systèmes appuyant le statu quo. Néanmoins, en même temps, il existe un potentiel pour que la diversification et la pluralisation des configurations des infrastructures concourent à des objectifs plus progressistes, façonnés par et pour des enjeux collectifs, et s'ouvrant plutôt que se fermant à la diversité des futurs sociotechniques urbains possibles.

La compréhension et la conceptualisation du changement sociotechnique doivent donc être au cœur même des analyses des processus d'urbanisation contemporains à l'échelle mondiale, comme elles sont au fondement des remaniements et des réarticulations constants des formes de territorialité aréolaires et relationnelles qui constituent ces processus.

\section{Références}

Bakker K. (2003), "Archipelagos and networks: urbanisation and water privatisation in the South ", Geographical Journal, vol. 169, n' 4, p. 328-341. 
Bijker W., Hughes T., Pinch T. (éds) (1987), The Social Construction of Technological Systems: New Directions in the Sociology and History of Technology, Cambridge (Mass.), The MIT Press.

Botton S., De Gouvello B. (2008), "Water and sanitation in the Buenos Aires metropolitan region: fragmented markets, splintering effects? ", Geoforum, n 39, p. 1859-1870.

Brenner N. (2013), "Theses on urbanization ", Public Culture, vol. 25, $\mathrm{n}^{\circ} 1$, p. $85-114$.

Castells M. (2010), "Globalisation, networking, urbanisation: reflections on the spatial dynamics of the information age ", Urban Studies, vol. 47, $\mathrm{n}^{\circ} 13$, p. 2737-2745.

Coutard O. (éd.) (2008), "Placing Splintering Urbanism ", Geoforum, vol. 39, n 6, p. 1815-1950.

Coutard O. (2010), "Services urbains : la fin des grands réseaux ?", dans Coutard O., Lévy J.-P. (éds), Écologies urbaines : état des savoirs et perspectives, Paris, Economica-Anthropos.

Coutard, O., Rutherford J. (éds) (2016), Beyond the Networked City: Infrastructure Reconfigurations and Urban Change in the North and South, Abingdon, Routledge.

Dupuy G. (1991), Lurbanisme des réseaux : théories et méthodes, Paris, Armand Colin.

Furlong K. (2011), " Small technologies, big change: rethinking infrastructure through STS and geography ", Progress in Human Geography, vol. 34, n 4, p. 460-482.

Gandy M. (2002), Concrete and Clay: Reworking Nature in New York City, Cambridge (Mass.), The MIT Press.

Gandy M. (2008), «Landscapes of disaster: water, modernity, and urban fragmentation in Mumbai ", Environment and Planning A, vol. 40, p. 108-130.

Geels F. W., Schot J. (2007), "Typology of sociotechnical transition pathways ", Research Policy, vol. 36, n 3, p. 399-417.

Graham S., Marvin S. (2001), Splintering Urbanism: Networked Infrastructures, Technological Mobilities and the Urban Condition, Londres, Routledge. 
Guy S., Marvin S., Moss T. (2001), Urban Infrastructure in Transition: Networks, Buildings, Plans, Londres, Earthscan.

Heynen N., Kaika M., Swyngedouw E. (éds) (2006), In the Nature of Cities: Urban Political Ecology and the Politics of Urban Metabolism, Londres, Routledge.

Hommels A. (2005), "Studying obduracy in the city: toward a productive fusion between technology studies and urban studies ", Science, Technology, \& Human Values, vol. 30, n 3, p. 323-351.

Hughes T. (1983), Networks of Power: Electrification in Western Society, 1880-1930, Londres, Johns Hopkins University Press.

Jaglin S. (2005), Services d'eau en Afrique subsaharienne : la fragmentation urbaine en question, Paris, CNRS Éditions.

Kaika M., Swyngedouw E. (2000), "Fetishizing the modern city: the phantasmagoria of urban technological networks ", International Journal of Urban and Regional Research, vol. 24, $\mathrm{n}^{\circ}$ 1, p. 120-138.

Keil R. (2003), "Urban political ecology », Urban Geography, vol. 24, n 8, p. 723-738.

Lorrain D. (éd.) (2011), Métropoles XXL en pays émergents, Paris, Presses de Science Po.

McFarlane C. (2008), " Governing the Contaminated City: Infrastructure and Sanitation in Colonial and Post-Colonial Bombay ", International Journal of Urban and Regional Research, vol. 32, $\mathrm{n}^{\circ}$ 2, p. 415-435.

McFarlane C., Rutherford J. (2008), « Political Infrastructures: Governing and Experiencing the Fabric of the City ", International Journal of Urban and Regional Research, vol. 32, n 2, p. 363-374.

Melosi M. (2000), The Sanitary City: Urban Infrastructure in America from Colonial Times to the Present, Londres, Johns Hopkins University Press.

Moss T. (2000), "Unearthing water flows, uncovering social relations: introducing new waste water technologies in Berlin ", Journal of Urban Technology, vol. 7, $\mathrm{n}^{\circ}$ 1, p. 63-84.

Offner J.-M. (2000), “"Territorial deregulation”: local authorities at risk from technical networks ", International Journal of Urban and Regional Research, vol. 24, n 1, p. 165-182. 
Pflieger G., Matthieussent S. (2008), "Water and power in Santiago de Chile: socio-spatial segregation through network integration ", Geoforum, vol. 39, p. 1907-1921.

Shove E. (2012), "The shadowy side of innovation: unmaking and sustainability ", Technology Analysis \& Strategic Management, vol. 24, $\mathrm{n}^{\circ}$ 4, p. 363-375.

Simone A. (2004), "People as infrastructure: intersecting fragments in Johannesburg ", Public Culture, vol. 16, n 3, p. 407-429.

Soja E. (2000), Postmetropolis: Critical Studies of Cities and Regions, Oxford, Blackwell.

Swyngedouw E. (2004), Social Power and the Urbanization of Water: Flows of Power, Oxford, Oxford University Press.

Tarr J. (1979), "Introduction ", Journal of Urban History, vol. 5, n 3, p. $275-278$.

Tarr J., Dupuy G. (éds) (1988), Technology and the Rise of the Networked City in Europe and America, Philadephie (Penn.), Temple University Press.

Walker G., Cass N. (2007), "Carbon reduction, "the public” and renewable energy: engaging with socio-technical configurations ", Area, vol. 39, $n^{\circ} 4$, p. 458-469. 


\title{
Politiques d'infrastructures en Afrique subsaharienne
}

\section{Le réseau est-il soluble dans la transition urbaine?}

\author{
Sylvy Jaglin
}

Les villes africaines sont-elles une variante des configurations infrastructurelles témoignant de l'émergence d'une ère urbaine " postréseau " (Coutard et Rutherford, 2013) ? En première approche, la question a l'avantage de proposer une vision alternative aux analyses dominantes du retard et des défaillances des infrastructures matérielles dans les villes africaines (Banerjee et Morella, 2011 ; Foster et BriceñoGarmendia, 2010 ; Eberhard et al., 2011 ; Lall et al., 2017), pour lesquelles les espaces urbains africains incarnent divers stades de villes pré-réseau. Elle permet aussi d'articuler entre eux de nombreux travaux soulignant la diversité et la spécificité des modes de fourniture des services essentiels urbains, dans un cadre largement hors-réseau (Botton et Blanc, 2014 ; Berthélémy, 2016 ; Katusiimeh et al., 2013 ; Jaglin et Zérah, 2010 ; Lorrain et Poupeau, 2014; Van Dijk et al., 2014). Illustrant comment diverses avancées technologiques couplées à la décentralisation croissante de la gestion urbaine permettent de contourner les blocages de l'extension du réseau intégré, ces travaux semblent faire écho aux évolutions qui résultent, au Nord, de la remise en cause du grand réseau. Celle-ci est suscitée par des évolutions macrosociales (faibles croissances économique, démographique et urbaine, ainsi que diverses contestations des formes d'organisation centralisées et hiérarchisées) et des préoccupations environnementales, qui s'accompagnent d'une injonction à plus de sobriété dans la consommation des ressources, laquelle peut aussi passer par une relocalisation des métabolismes urbains, des infrastructures décentralisées et des symbioses réticulaires (Coutard et Rutherford, 2013). Qu'apporte toutefois la notion de 
post-réseau pour analyser et comprendre les situations africaines, qui associent depuis longtemps réseau conventionnel et dispositifs horsréseau dans des configurations de fourniture composites (Jaglin, 2005, 2014) ? Au-delà d'une lecture des paysages infrastructurels, premier palier de la compréhension, les analogies permettent-elles de rendre compte des évolutions infrastructurelles les plus significatives?

Dans une approche relationnelle des infrastructures, pensées comme à la fois façonnées par et façonnant les contextes sociaux dans lesquels elles sont insérées, ce chapitre défend le parti d'une analyse contextualisée des coévolutions entre services urbains et transitions urbaines en Afrique. Il considère ainsi les configurations de fourniture comme des assemblages urbains constitués d'acteurs, d'objets techniques, d'institutions, d'intérêts économiques, de pratiques et représentations sociales, mais aussi d'espaces. Ces assemblages incorporent donc les caractéristiques des contextes urbains africains : leur croissance démographique soutenue parmi les plus fortes du monde (3,7\%/an de 2000 à 2010), leur hétérogénéité socio-économique croissante (Hugon, 2014 ; Lautier, 2004), leur pluralité de normes et de règles (Chauveau et al., 2001), leurs hybridations techniques. Peu propices à l'homogénéisation des cadres et conditions de l'action collective, ces caractéristiques semblent favoriser des assemblages durablement hétérogènes plutôt que des logiques de substitution ou de transition d'une ville pré-réseau vers une ville d'aprèsréseau.

Dans la recherche, ces assemblages - configurations de fourniture (Olivier de Sardan, 2010), modernised mixtures (Vliet et al., 2014), coproduction (Ahlers et al., 2014 ; Moretto et Allen, 2015) - sont analysés sous l'angle des théories du changement infrastructurel (Furlong, 2014), des modes de gouvernance en contextes hétérogènes et de pluralité normative (Baron et Bonnassieux, 2013), du rôle et de la nature de l'" informel " (Ahlers et al., 2013 ; Ahlers et al., 2014 ; Botton et Blanc, 2014). Apparaissant comme des «solutions de second choix [qui] semblent offrir un heureux compromis " (Foster et BriceñoGarmendia, 2010, p. 98), ils sont en outre à présent déclinés dans divers formats opérationnels (Berthélémy et Béguérie, 2016 ; Botton et Blanc, 2014 ; Naulet et al., 2014 ; Tsitsikalis, 2011). Cette reconnaissance témoigne d'une inflexion marquante de la manière de penser et produire les services urbains dans ces villes, dont nous retenons ici qu'elle engage 
des processus de construction institutionnelle et de renégociation de la manière dont les appareils d'État africains (à toutes les échelles) interagissent avec les acteurs (notamment non conventionnels) des services urbains. De quoi cette inflexion est-elle significative ? Est-elle le moteur d'un nouveau régime sociotechnique urbain (Bulkeley et al., 2014) dans les villes africaines?

Le chapitre part de deux hypothèses constitutives de ce que nous proposons d'appeler un «tournant pragmatique ». La première est que la reconnaissance croissante des configurations de fourniture hybrides et leur intégration actuelle dans la pensée urbanistique sont une réponse à de nouvelles dynamiques urbaines : d'une part, l'étalement rapide sous forme d'un continuum urbain-rural hétérogène rend vain tout espoir de rattrapage à moyen terme par les réseaux; d'autre part, l'émergence des " classes moyennes ", catégorie floue et discutée (Darbon et Toulabor, 2014), est associée, depuis les années 2000, à des transformations de la demande et des pratiques de consommation des services urbains. La seconde hypothèse est que la formalisation des configurations de fourniture constitue un mode expérimental de régulation de la transition urbaine. La notion de régulation renvoie ici à deux registres d'action emboîtés : la régulation sectorielle des services, ensemble des facteurs techniques, économiques, juridiques et organisationnels qui en régissent le fonctionnement ; la régulation politique, entendue comme l'ensemble des mécanismes auxquels recourent les pouvoirs publics pour stabiliser les antagonismes et assurer la reproduction d'un système social (Jaglin, 2007). Cet emboîtement étant précaire, le mode de régulation reste lui-même instable et contingent.

La première partie analyse les configurations de fourniture et les politiques de formalisation en interrogeant leur contribution à une éventuelle transition infrastructurelle vers une ville post-réseau en Afrique. La seconde partie resitue ces évolutions dans les contextes sociospatiaux de la transition urbaine africaine et souligne les enjeux de régulation des configurations de fourniture hybrides, d'abord pensées comme une réponse pragmatique et marchande à l'hétérogénéité urbaine. 


\section{De l'informalité à la régula(risa)tion des configurations de desserte}

Améliorer les conditions d'accès aux services essentiels dans les villes africaines à forte croissance démographique et spatiale reste un défi auquel les politiques et les projets mis en ouvre ces dernières décennies ont répondu avec un succès très limité. Les opérateurs des réseaux conventionnels ne sont pas parvenus à forger des offres adaptées aux besoins comme au pouvoir d'achat de la majorité des citadins. Moins de $40 \%$ des ménages urbains africains ont l'eau courante à domicile, une fosse septique ou des latrines améliorées et, dans près de la moitié des pays africains, l'électricité atteint à peine $50 \%$ des citadins, tandis que la poussée démographique en zone urbaine a fait chuter les taux de couverture de tous les services urbains (notamment la fourniture d'eau potable) entre 1990 et 2005 (Foster et Briceño-Garmendia, 2010, p. $126-127)^{24}$.

L'offre de services essentiels est aujourd'hui marquée par trois données structurelles. D'abord, dans tous les secteurs, la demande est en forte croissance, plus encore depuis que l'urbanisation est accompagnée d'une consolidation des classes moyennes. Ensuite, l'accès aux ressources et aux services demeure rationné : une importante demande latente reste donc à combler, ce qui est à la fois une source d'opportunités économiques, comme en attestent l'intérêt et les convoitises pour ces nouveaux marchés (Damon 2014 ; Desjeux 2011), et un facteur de risque pour les autorités urbaines et les États. Enfin, la question politique d'une démocratisation de l'accès aux ressources urbaines (et du droit à la ville) reste profondément marquée par une économie morale dans laquelle le clientélisme et le patronage sont des formes contestées, mais relativement efficaces, de partage des ressources au sein de sociétés inégalitaires.

\section{Du réseau aux configurations de fourniture}

Les configurations de fourniture juxtaposent un réseau conventionnel, en fonction duquel les politiques publiques pensent et organisent la régulation, et un ensemble hétérogène d'offres informelles. La gestion

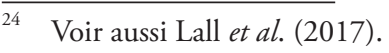


du réseau a d'abord été dominée par des acteurs publics et une régulation étato-centrée puis, à partir des années 1990, les réorganisations orchestrées dans le cadre de réformes néolibérales ont favorisé la marchandisation, les contrats de partenariat avec des entreprises privées et l'introduction de divers dispositifs de concurrence. Lente et circonscrite, la diffusion de ces partenariats public/privé n'a apporté que des réponses très partielles à la demande urbaine qui reste en partie dépendante d'opérateurs publics inégalement modernisés et, surtout, d'opérateurs informels. Les offres marchandes non conventionnelles, individuelles ou collectives, formelles ou informelles, sont d'initiative privée et illégales au regard des contrats d'exclusivité des opérateurs officiels. Exploitant la diversité des demandes et des capacités à payer comme celle des conditions citadines, elles pallient la déficience du service conventionnel et s'adressent, selon les types d'espaces urbains, à des clientèles aisées insatisfaites du service ou à des clientèles pauvres, exclues en raison de leur faible pouvoir d'achat, de leur éloignement géographique ou de l'illégalité de la tenure du sol (Jaglin, 2012, 2014).

Les exemples sont multiples dans tous les domaines : les marchés de l'eau ont été abondamment décrits (voir le dossier de Water Alternatives 2014-7(1) ; Baron et Bonnassieux, 2013 ; Botton et Blanc, 2014 ; Jaglin 2005, 2013 ; Naulet et al., 2014) ainsi que les offres informelles de transport (Godard, 2002 ; Lombard, 2006 ; Pradeilles et al., 1991 ; Rizzo, 2011). L'inscription des solutions privées d'assainissement dans des chaînes de valeur assurant leur financement durable est l'objet d'une attention croissante (Trémolet et Evans, 2010 ; Van Dijk et al., 2014) ; la collecte des déchets et les diverses filières de récupération et recyclage sont également au cœur de nombreuses réflexions (Ginisty, 2014 ; Katusiimeh et al., 2013 ; Ngambi, 2015 ; Pierrat, 2014 ; Sory, 2013). Moins connus, les services énergétiques alternatifs sont en plein essor (Africa Progress Panel, 2017 ; Berthélémy, 2016 ; Franks et Prasad, 2014; Gaunt et al., 2012 ; Lawaetz et Smyser, 2011) tandis que l'inventivité des services de télécommunication et le développement d'un véritable écosystème mobile africain sont désormais attestés (Cheneau-Loquay, 2012 ; GSMA, 2016). Ces systèmes ne sont pas infinis en nombre et en variété ; tous n'existent pas partout ni avec la même intensité, mais dans toutes les villes, le pluralisme technique et gestionnaire des configurations 
de fourniture est le résultat d'une co-construction circonstancielle permettant d'étendre des fonctionnalités de service jusqu'à un usager final non ou partiellement connecté au réseau conventionnel.

Ce sont ces configurations que les politiques actuelles tentent de formaliser voire, lorsqu'elles n'existent pas, de susciter. Elles comportent trois principaux volets : l'organisation, la professionnalisation et parfois la concentration des petits opérateurs privés ; la coordination, dans l'espace urbain et dans le temps, de l'ensemble des offres ; la régulation des services (tarifs, qualité, concurrence) et des conflits éventuels. Plus encore que la pertinence technique de leurs approches, ces politiques dépendent, pour leur mise en ouvre, d'institutions publiques fragiles, encore marquées par la prégnance des héritages coloniaux et des processus de transferts institutionnels et cognitifs emblématiques des "sociétés projetées " (Darbon, 2008). Si la capacité de régulation des pouvoirs publics est ainsi souvent faible et la capacité à gouverner l'informel plus encore, elle n'est pas nulle. Il s'agit donc d'analyser l'évolution des rapports de force, en fonction des bénéfices attendus et des risques encourus ${ }^{25}$.

Dans le service des déchets, les "schémas globaux " municipaux associent une pré-collecte confiée à des opérateurs informels, parfois couplée à des activités de tri et valorisation, un service municipal d'enlèvement et de transport à partir des stations de transfert, des centres d'enfouissement contrôlés, dont la gestion est de plus en plus souvent déléguée à une entreprise privée sous contrat. De nombreux travaux (Katusiimeh et al., 2013 ; Ngambi, 2015 ; Pierrat, 2014 ; Sory, 2013) soulignent qu'un des enjeux de cette organisation tient à la coordination, sur l'ensemble de la chaîne, d'acteurs hétérogènes dont l'activité n'est unifiée ni par les pratiques et représentations, ni par les

25 La démarche s'inspire ici de Schon et Altrock qui, dans des contextes urbains chinois, proposent le concept de " conceded informality " pour analyser " a flexible management of diverse informal practices depending on their relevance, usefulness and potential threat towards state authority" (Schon et Altrock, 2014, p. 216). À travers cinq stratégies, décrites comme des "modes of governing "the informal" ", les auteurs montrent comment l'État combine deux nécessités : trouver des solutions pratiques et opératoires aux transformations urbaines et économiques rapides ; gérer le passage d'un parti-État dominant à un parti-État en quête de légitimation et de formes de coopération avec des acteurs non étatiques pour gouverner les villes (ibid.). 
dispositifs techniques et réglementaires, ni par les résultats. Dans le domaine de l'eau, où les marchés urbains sont à la fois anciens et très divers, différentes expériences sont en quête d'arrangements adéquats. Certaines privilégient la délégation à des opérateurs privés, à la fois " clients grands comptes » de l'opérateur conventionnel et « détaillants » pour les habitants (Botton et Blanc, 2014, p. 112), d'autres tentent d'encadrer et de réguler des producteurs-distributeurs indépendants (Ahlers et al., 2013 ; Botton et Blanc, 2014 ; Valfrey-Visser et al., 2006) ou des mini-réseaux confiés en gestion à des associations d'usagers (Bédécarrats et al., 2016 ; Naulet et Biteete, 2014). Dans celui de l'assainissement, la réflexion est plus balbutiante et s'efforce d'identifier les segments de l'activité sur lesquels l'action publique pourrait intervenir avec efficacité (Trémolet et Evans 2010). À propos de l'assainissement de deux quartiers irréguliers de Dar es-Salaam et Kampala, Van Dijk et al. identifient ainsi trois segments fonctionnels - construction, entretien et vidange des latrines - et suggèrent que les principaux verrous du développement d'un service satisfaisant pourraient être levés par une politique pragmatique de reconnaissance des solutions existantes, de développement technologique et de formation des artisans locaux (Van Dijk et al., 2014).

Dans tous ces exemples, les politiques de formalisation visent à encadrer les activités de service informelles et à les coordonner avec celles de l'opérateur conventionnel, notamment à travers une nouvelle ingénierie institutionnelle de délégation ou de micropartenariat. En sécurisant les marchés de services, y compris au prix de "bricolages sociotechniques »(Criqui, 2015), elles les ouvrent à de nouveaux entrepreneurs et de nouveaux consommateurs, notamment des classes moyennes, plus exigeants sur la qualité et la fiabilité des offres.

\section{De la formalisation à la transition infrastructurelle?}

Formels ou informels, les services essentiels étudiés sont produits à l'aide de dispositifs sociotechniques associant des artefacts techniques, des organisations sociopolitiques, des savoirs, mais aussi des pratiques et des représentations. Certains dépendent d'une infrastructure matérielle en réseau " lourde " et capitalistique, d'autres s'appuient sur des réseaux d'acteurs et de technologies dispersées mobilisant un faible capital fixe. Considérés ensemble à l'échelle des configurations de fourniture, 
ces dispositifs sociotechniques ne partagent en revanche ni une infrastructure, au sens de Star et Ruhleder $(2010)^{26}$, ni des conventions propres à solidifier des communautés de pratiques. Les politiques et projets de formalisation de ces configurations hybrides ont précisément pour objectif de faire émerger l'une et les autres.

On peut dès lors interroger leur contribution à une transition infrastructurelle urbaine (Bulkeley et al., 2014 ; Rutherford et Coutard, 2014) conduisant in fine à une ville post-réseau. Commençons par trois constats inspirés par l'analyse des transitions sociotechniques, ici adaptée aux contextes urbains des Suds (Furlong, 2014). D’abord, ce n'est pas tant la critique des grands systèmes techniques que l'objectif de " mise en réseau » des dispositifs de fourniture hétérogènes existants qui domine. Ensuite, les changements examinés procèdent moins de logiques de substitution que d'une série d'adaptations incrémentales, facilitant l'intégration fonctionnelle, relative et inégale, d'acteurs et de modalités de desserte longtemps encapsulés dans des mondes sociotechniques étanches ${ }^{27}$. Enfin, ce mouvement ne s'accompagne nullement d'une contestation du modèle, des valeurs et pratiques dominantes. De nombreuses études montrent au contraire, en Afrique (Kjellen et McGranahan, 2006 ; Ginisty 201428) et ailleurs dans les Suds (Criqui, 2015), que le modèle du réseau n'est pas remis en cause par les alternatives. Loin de l'éliminer, la somme des bricolages le consolide comme référence autour de laquelle s'organisent les autres dispositifs (Criqui, 2015 ; Jaglin, 2005). Pour réconcilier les cadres conceptuels des études sociotechniques avec les conditions qui

${ }_{26}$ Ces auteurs appréhendent l'infrastructure comme un concept fondamentalement relationnel, renvoyant à des pratiques organisées : " une infrastructure est une propriété relationnelle et non un objet sans usage " (Star et Ruhleder, 2010, p. 118).

27 Au moins dans le cadre des politiques sectorielles et dans l'exercice officiel des pratiques professionnelles. Nombre d'études ont en revanche souligné que les « plombiers du dimanche " et autres " braconneurs » d'électricité partagent leur temps entre travail formel dans les entreprises de service et bricolage informel à la demande, établissant ainsi entre ces deux mondes un pont très antérieur à sa prise en compte dans les politiques.

28 À Maputo, les ménages interrogés par Ginisty (2014) choisissent les petits opérateurs plutôt que les bornes-fontaines, mais ils témoignent aussi de la résistance du modèle conventionnel du branchement particulier, horizon d'une citadinité " aboutie ", pour lequel ils consentent ou ont consenti d'importants efforts financiers. 
prévalent dans les villes des Suds, Furlong suggère d'admettre qu'un système sociotechnique stable peut être durablement hybride et que la transition infrastructurelle peut consister en un processus continu de diversification et d'hétérogénéisation (Furlong, 2014).

La notion de transition semble néanmoins imparfaite pour saisir deux caractéristiques structurantes des dynamiques internes aux configurations de fourniture : la « résilience » du modèle du grand réseau et la capacité de son infrastructure réticulée à cohabiter avec une diversité croissante de solutions " alternatives». Ainsi, la diffusion de technologies et de produits chinois à bas coût sur les marchés africains a transformé les conditions techniques de fourniture de tous les services. Batteries électriques, panneaux photovoltaïques, compteurs, cuves et tuyaux en polyéthylène, mais aussi téléphones mobiles d'entrée de gamme et d'occasion témoignent de l'introduction réussie de technologies low-cost qui ont facilité, encouragé ou accéléré l'émergence de petits opérateurs privés et diversifié les dispositifs d'accès (mini-réseaux d'eau, kiosques énergétiques, installations individuelles comme les inverters au Nigeria ou les chauffe-eau solaires en Afrique du Sud). Paradoxalement, en compensant les dysfonctionnements récurrents des réseaux, ces innovations les rendent aussi plus acceptables ou supportables et, dans le contexte d'un marché croissant, contribuent à atténuer la pression sur le réseau conventionnel plus qu’à en bouleverser ou en menacer le fonctionnement.

En outre, les infrastructures du grand réseau technique conservent un fort pouvoir d'attraction. Les discours des techniciens et des ingénieurs l'illustrent, mais aussi ceux des ménages. Ces représentations sont confortées par les faits, car les réseaux conventionnels poursuivent leur lente conquête des territoires, au gré des opportunités d'investissement, dans un mouvement continu d'infrastructuralisation (Chatzis, dans cet ouvrage ${ }^{29}$. Par extensions nouvelles ou par adjonction/intégration de dispositifs locaux hétérogènes préexistants, ils accompagnent avec

29 Chatzis définit l'infrastructuralisation comme « le processus de transformation de dispositifs locaux en infrastructures, via la stabilisation/pérennisation de leur fonctionnement et leur extension/généralisation par la reproduction de la même unité de base à des échelles spatiales et sociales toujours plus grandes et/ou par la combinaison/interconnexion de plusieurs systèmes différents " (Chatzis, Introduction générale. Le nouveau monde des infrastructures). 
retard l'urbanisation des territoires en provoquant alors le reflux des alternatives décentralisées : celles des comités de gestion des miniréseaux d'eau à Khartoum (Crombé, 2017), celle des petits opérateurs privés indépendants à Maputo (dont diverses modalités d'éviction négociée ont été scénarisées en anticipation des projets d'extension du réseau conventionnel : Blanc et al., 2009 ; Botton et Blanc, 2014), celle des revendeurs informels d'électricité au Cap (Franks et Prasad, 2014 ; Gaunt et al., 2012). Au sein des configurations de fourniture, ces évolutions différenciées favorisent l'imbrication croissante d'offres techniques hétérogènes. Au stade actuel, toutefois, les dispositifs informels sont rarement une solution alternative affranchie du réseau et les modifications partielles et réversibles des configurations de fourniture n'ont pas enclenché une transformation substantielle du régime sociotechnique urbain du réseau; bien au contraire, elles ont préservé son existence en comblant les vacances des calendriers distendus de l'infrastructuralisation en Afrique, comme le montrent Andreasen et Møller-Jensen (2016) dans les périphéries de Dar es-Salaam.

Certes, les rythmes d'extension du réseau sont lents et les progrès de la couverture partiels au regard des territoires urbanisés mais, sur le temps long de la fabrique urbaine, on ne constate ni récession ni atrophie des grands réseaux. On observe en revanche une complexification de leurs architectures, qui combinent plus ou moins étroitement des portions de grands systèmes techniques conventionnels, aux infrastructures matérielles discontinues, des sous-systèmes réticulaires greffés aux grandes infrastructures, débranchables à la demande et disposant d'une certaine autonomie de gestion (électricité, eau, déchets), des dispositifs individuels, fonctionnellement autonomes ou supplétifs du réseau (batteries alimentées par panneaux photovoltaïques et réseau électrique, cuves de stockage de l'eau alimentées par forage et canalisations), mais souvent dépendants d'une logistique " en réseau " (commerce de location de batteries, colportage et revente d'eau à domicile).

C'est donc moins la transformation et la substitution de régimes sociotechniques que la manière dont ils coexistent et co-évoluent qui est au cœur du problème. La question centrale porte ainsi sur les conditions d'articulations " efficaces » entre eux, c'est-à-dire d'un travail 
de coordination (Star et Ruhleder, 2010) susceptible de produire un agencement pertinent entre ces mondes hétérogènes. C'est tout l'enjeu des régulations observables.

\section{Régulations marchandes en contextes urbains hétérogènes : vers un tournant pragmatique ?}

Cette seconde partie défend l'idée que les configurations de fourniture hybrides décrites précédemment sont modelées par les conditions de l'urbanisation en Afrique (notamment l'étalement rapide et l'émergence de nouvelles demandes et pratiques de consommation en provenance des " classes moyennes ") et que leur formalisation constitue un mode expérimental de régulation de la transition urbaine par les pouvoirs d'État, que nous appelons « tournant pragmatique ».

\section{Émergence des classes moyennes, étalement urbain et nouveaux schémas sociospatiaux de la demande en service}

Les configurations de fourniture ont d'abord été associées à la demande des citadins pauvres, mais un double mouvement en influence aujourd'hui profondément les contenus : l'essor des classes moyennes urbaines et l'étalement spatial à l'africaine.

Floue et discutable, la notion de classe moyenne est au centre de débats dans les villes africaines, où la constitution de nouvelles formations sociales ascendantes est empiriquement observée (Darbon et Toulabor, 2014). Cette catégorie engloberait des millions de citadins aux revenus monétaires compris entre 2 et 20 US\$ par personne et par jour, qui ont en commun une double caractéristique : celle " de la sortie de la précarité fondée sur une capacité à dégager un surplus et [celle] d'une capitalisation insuffisante pour pouvoir arrêter efforts et vigilance, ce qui suggère la persistance d'une forme de vulnérabilité (Darbon, 2014, p. 44). Plus précisément, les classes moyennes, en Afrique, regrouperaient trois sous-catégories hétérogènes : les classes moyennes enrichies (10 à 20 US\$/p/jour, parfois qualifiées de Global Middle Class), à revenus substantiels et patrimoine conséquent ; les classes moyennes stabilisées (4 à 10 US\$/p/jour), qui se sont éloignées de la relégation 
dans la pauvreté, mais dont le patrimoine limité ne garantit pas une ascension sociale intergénérationnelle; les classes moyennes de "petite prospérité » aussi qualifiées de floating class (2 à 4 US\$), qui disposent d'un revenu discrétionnaire (disposable income), mais restent menacées de déclassement (Darbon, 2014).

Quoique dans des proportions variables, ces populations disposent d'un pouvoir d'achat et aspirent, en Afrique comme ailleurs, à consommer (Desjeux, 2016), cherchant en particulier à accéder de manière individualisée aux services urbains marchands pour conforter leur nouvelle position sociale, l'un des problèmes posés étant celui des modalités de satisfaction de cette demande. En effet, si la notion de " moyennisation " mathématique des revenus (Damon, 2014) a le mérite de souligner une dynamique continentale, elle n'est nullement synonyme d'une homogénéisation des comportements et valeurs, l'agrégation statistique masquant des particularismes nationaux et locaux. "Nom de code " plus que concept (Darbon, 2014), la notion de classes moyennes permet néanmoins d'attirer l'attention sur une double articulation entre changement social urbain et pratiques de consommation des services. D'abord, ces classes moyennes " s'inventent " à partir des représentations qu'elles se font d'ellesmêmes et de leur promotion sociale, dans lesquelles les désirs et normes de consommation sont importants et valorisent, notamment, l'individualisation de l'accès. Ensuite, l'amélioration du niveau de vie de ces classes moyennes doit beaucoup aux biens et services produits par l'économie informelle et à l'arrivée massive sur les marchés et dans la vie quotidienne de technologies low-cost en provenance de Chine et d'Inde (Darbon, 2014). En effet, les prix et modalités de commercialisation des offres (à l'unité et sous forme d'achats fractionnés) contribuent directement à l'actualisation des aspirations de ces classes sociales en leur proposant des prestations " accessibles" (même si elles ne sont pas moins chères). Les politiques de formalisation du tournant pragmatique sont ainsi une reconnaissance politique de la contribution des offres informelles à "l'invention " des classes moyennes, en popularisant l'accès à des services marchands et individuels, y compris hors réseau, et à leur " actualisation ", en commercialisant les services sous une forme qui solvabilise la demande citadine. 
Ces nouveaux marchés urbains sont en partie localisés dans les périphéries urbaines où se déploie une intense activité foncière et immobilière. Décrits comme des " conurbations multipolarisées " en bordure du golfe de Guinée (Dorier-Apprill et Domingo, 2004), du "rural-sprawl " (Harre et al., 2015) ou une forme de "desakota à l'africaine " (Calas, 2007) en Afrique de l'Est, ou encore une new African suburbanisation (Buire, 2014), ces espaces bâtis distendus et discontinus s'accompagnent d'une dilution du peuplement qui accroît les coûts d'infrastructure par habitant. Pour certains observateurs, le coût du déploiement de l'infrastructure de réseau devient même inabordable et il faut envisager des technologies alternatives moins chères telles que les panneaux solaires, les forages ou l'assainissement in situ (Foster et Briceño-Garmendia, 2010). Le problème, toutefois, n'est pas tant de mobiliser ces technologies que de les insérer dans des dispositifs sociotechniques appropriés et pérennes, d'autant que ces espaces sont le plus souvent situés hors du territoire de compétence de l'opérateur conventionnel, voire d'une autorité publique urbaine. Les configurations de fourniture y déclinent ainsi diverses formules opérationnelles, en fonction des ressources mobilisables par les promoteurs immobiliers et les occupants, entre enclaves riches autonomes hyperéquipées et vastes espaces livrés aux bricolages (Allen, 2014).

Dans ces espaces périurbains, la compétition pour l'accès au sol et aux ressources (l'eau notamment) est aiguë. Qu'on l'observe à travers les nouvelles utopies de la planification (Watson, 2014), les promesses de l'urbanisme entrepreneurial (Myers, 2015) ou encore la production d'éco-enclaves immobilières exclusives (Allen, 2014), le renouveau des politiques urbaines en témoigne. La vision d'un affrontement dual entre de riches accapareurs et des populations marginalisées, dépossédées ou déplacées (ibid.), est cependant réductrice et sous-estime à la fois le rôle des classes moyennes émergentes dans ces espaces convoités et l'économie politique de la production urbaine associée à cette demande.

En tant qu'actrices de la ville, les classes moyennes en émergence ont en effet une double caractéristique : elles sont politiquement discrètes et très directement investies dans l'amélioration de leurs conditions de vie. Ceci résulte, selon Darbon, d'un conformisme et d'un "individualisme forcené lié à la poursuite de trajectoires de promotion exigeantes " et exposées à quatre tensions : économique 
(contre la précarité), psychologique (pour se discipliner), sociale (pour rompre les anciens liens sociaux), normative (pour adopter les règles et normes des classes riches) (Darbon, 2014, p. 48-49). Pourtant, leur manque d'investissement et de maîtrise de l'espace public comme la rareté des mobilisations collectives des classes moyennes africaines ne témoignent pas d'un retrait de la sphère politique mais d'une participation assurée par d'autres moyens : "Les groupes dits de "classe moyenne" sont soit cachés derrière le pouvoir d'État qui leur permet par action ou par laisser-faire de poursuivre leurs efforts de promotion, soit largement portés par lui »(Darbon, 2014, p. 48). Autrement dit, l'énergie des classes moyennes émergentes est, pour le moment, tout entière tendue vers l'aménagement de leur cadre de vie, la satisfaction de leur désir de consommation et la réalisation de leur rêve urbain.

Or, ni les services publics défaillants, ni les offres de marché formelles, trop coûteuses, ni les seuls petits opérateurs informels ne peuvent, isolément, supporter ce mouvement. Il faut trouver de nouveaux arrangements économiques et politiques viables pour satisfaire la demande de ces citadins, sortis de la pauvreté et désireux de payer pour des services améliorés, courtisés par des leaders politiques locaux en quête de bases sociales dans la compétition électorale locale.

Les politiques du «tournant pragmatique " sont ainsi une réponse à la transition urbaine, à la fois dans sa dimension socio-économique et politique. Cette dernière est capitale : bien plus que les citadins très pauvres, accaparés par des stratégies de survie au jour le jour, et que les ménages aisés ou riches, que leur pouvoir d'achat et leur mobilité conduisent à trouver satisfaction sur les marchés globalisés, les petites couches moyennes sont aujourd'hui demandeuses d'action publique. Leurs exigences en termes de qualité du cadre de vie et de confort domestique ne sont pas pleinement satisfaites par les offres marchandes disponibles, tandis que leurs modes de vie comme leurs aspirations les détournent de solutions d'entraide et d'autoproduction dont elles cherchent à s'émanciper. Les opérateurs conventionnels ne pouvant et ne sachant pas répondre seuls à ces demandes urbaines, que la seule différenciation des prestations ne peut satisfaire (Jaglin, 2008), nous 
suggérons que des politiques urbaines "pragmatiques ", par empilement et hybridation au sein des configurations de fourniture, sont en quête de réponses à la pluralité des demandes.

En puisant dans le répertoire des politiques urbaines néolibérales - formalisation des règles, extension du marché, valorisation des logiques entrepreneuriales et de la concurrence -, elles troquent un contrôle accru de l'État sur une partie des activités informelles contre des protections juridiques et politiques. Ce faisant, elles ouvrent aussi de nouveaux espaces d'opportunités aux consommateurs et producteurs des classes moyennes émergentes, canalisant leur énergie contestataire vers l'exploration économique de foisonnants marchés de services.

\section{Un pilotage étatique à géométrie variable}

Que la formalisation des configurations de fourniture soit une réponse adaptée aux demandes urbaines ne signifie cependant pas qu'elle soit sans problèmes (Ahlers et al., 2014 ; Jaglin, 2014). En l'absence d'économies d'échelle et de standardisation des prestations, les services informels sont souvent coûteux pour les ménages de la floating class, menacés d'exclusion par des taux d'effort insoutenables ; faute de modalités appropriées de contrôle et de régulation technique, la qualité et la continuité des services sont à la fois inégales et variables ; la solidarisation du corps social et des territoires n'est pas assurée par les marchés et suscite des craintes formulées en termes de fragmentation urbaine ; les externalités environnementales et sanitaires ne sont pas prises en charge par les acteurs privés informels, entraînant des risques de surexploitation des ressources collectives au détriment des équilibres écologiques d'ensemble.

Ainsi, la cartellisation des services d'eau (Jaglin et Bousquet, 2012), les ententes entre pré-collecteurs des déchets (Sory, 2013) comme le corporatisme des syndicats dans les taxis informels (Pradeilles et al., 1991; Wilkinson, 2010) attestent des limites de la concurrence imparfaite sur les marchés informels pour réguler non seulement les conditions d'accès des citadins au service, mais aussi les relations de travail au sein des unités de production (Rizzo, 2011), la compétition entre producteurs inégaux et plus encore les externalités : surexploitation des nappes phréatiques, rejets incontrôlés des déchets dans les interstices urbains, congestion des 
rues... Une étude sur les collecteurs informels de déchets à Kampala insiste sur le contraste entre leur prestation d'enlèvement, appréciée des ménages, et leur totale inefficacité environnementale (Katusiimeh et al., 2013). Une autre étude, sur les ventes d'eau à partir de forages privés à Nairobi, relève également les limites du service en termes d'équité sociale et de préservation de la ressource, les pauvres achetant une eau de qualité incertaine à un prix plus de 10 fois supérieur à celui que paient les ménages riches pour la première tranche d'eau du réseau, tandis que la surexploitation des eaux souterraines, dont la gestion est inefficace, conduit à un affaissement rapide du niveau de la nappe dans certains quartiers (Chakava et al., 2014).

Ces défaillances ne résultent pourtant ni du caractère hybride des configurations, ni du statut privé des opérateurs, ni, intrinsèquement, du caractère informel de leurs activités. Il faut en chercher l'origine dans la nature du pilotage politique. Trois exemples permettront de l'illustrer.

À Ouagadougou, vingt ans après le compromis politique sankariste de la "gestion partagée ", dont l'épuisement était perceptible dès le début des années 1990 (Jaglin, 1995), la politique de délégation du service de l'eau à des petits opérateurs privés sous contrat avec l'Office national de l'eau et de l'assainissement (ONEA) assure la desserte de 86000 périurbains (Botton et Blanc, 2014). Cette configuration témoigne d'une politique de l'opérateur principal soucieux de prolonger son action aux marges du réseau conventionnel sans perdre le contrôle du service de l'eau : en s'appuyant sur de petits opérateurs sous contrat, il marginalise d'autres alternatives d'accès (notamment la revente par colportage à partir des bornes-fontaines) et assure une relative homogénéité des principes du service (qualité de l'eau, grille tarifaire) tandis que les spécifications techniques et la courte durée des contrats (5 ans) maintiennent la perspective d'une intégration future au réseau.

Ce modèle diffère de celui qui prévaut lorsque l'organisation de la configuration de fourniture est d'abord fondée sur les principes d'un marché concurrentiel, censé sélectionner les meilleures offres et pratiques. Ahlers et al. (2013) critiquent néanmoins " the myth of "healthy" competition " qui sous-tend ces politiques de formalisation. Ces auteurs montrent, dans l'exemple de Maputo, que les fortes inégalités de taille et de puissance économique des petits opérateurs informels indépendants contredisent l'idée d'un marché " ouvert " : comme dans le secteur 
conventionnel de l'eau, " the combination of sunk costs and economies of density leads to monopoly-like characteristics " (Ahlers et al., p. 181). En outre, les défaillances de marché sont aggravées par les régulations (sur les prix, les conditions d'entrée sur le marché et d'extension spatiale des mini-réseaux) mises en œuvre par les deux associations d'opérateurs, dominées par les plus gros d'entre eux, qui imposent des logiques de cartel au détriment des intérêts des usagers.

À Douala (Nantchop, 2017), le service d'eau est officiellement assuré depuis 2008 par la Camerounaise des Eaux (CDE), société privée titulaire d'un contrat d'affermage. La détérioration continue du service et le recul du taux de couverture, par manque d'investissement dans les extensions et le renouvellement des infrastructures, ont toutefois favorisé le développement parallèle d'un entrepreneuriat privé de l'eau à la fois producteur (à partir de forages dans la nappe phréatique) et distributeur (bornes-fontaines, mini-réseaux et revente d'eau en bouteilles et sachets). La coordination entre tous ces opérateurs de l'eau n'est cependant pas assurée. La communauté urbaine de Douala serait favorable à la coexistence des opérateurs formels et informels, mais la CDE y est hostile ; l'État a créé un cadre juridique libéralisant la distribution de l'eau potable, mais entretient une concurrence interministérielle dans la régulation de l'exploitation de l'eau à des fins commerciales, concurrence qu'exploitent les opérateurs privés, dans une compétition économique et territoriale de plus en plus âpre, à travers deux « syndicats " concurrents. L'un représente des membres influents des élites sociales et économiques, proches de l'appareil d'État (membres de la diaspora, cadres du parti, hommes et femmes d'affaires) et dressés contre l'opérateur conventionnel et son monopole sur la ressource. L'autre rassemble les " petits » opérateurs dans un syndicat moins influent, qui ne peut compter sur un dialogue direct avec le pouvoir d'État et dépend des autorisations d'exploitation délivrées par un ministère qui les a aussi encouragés à s'organiser (Nantchop, 2017). En entérinant la rupture du monopole public de la distribution d'eau potable par une loi de 1998, l'État camerounais a servi les intérêts de quelques "Grands » tout en favorisant les activités de nombreux "petits » entrepreneurs informels de l'économie de l'eau urbaine (importation et vente de matériel chinois, travaux d'exécution des forages, laveries, vente d'eau ensachée ou embouteillée...). L'absence de régulation sectorielle conforte ici une régulation politique qui consiste, pour le pouvoir, à 
ouvrir de nouvelles opportunités de rente aux élites sans s'aliéner le petit entrepreneurariat urbain, dont la vitalité assure des services et des emplois pour les classes moyennes émergentes.

La formalisation des configurations de fourniture épouse ainsi des contours très divers : là où l'État burkinabè initie, contrôle et réglemente, les acteurs publics mozambicains cèdent la " police " du marché de l'eau à un "shadow regulator " plus soucieux des intérêts des gros producteurs que de ceux du public (Ahlers et al., 2013), tandis que la libéralisation camerounaise entretient, par un ensemble d'arrangements liant acteurs privés et agents de l'État, des coalitions redistributives aussi peu propices à une "normalisation " de l'économie de l'eau qu'essentielles aux arbitrages politiques actuels de l'État camerounais (Nantchop, 2017). Comment interpréter ces écarts ? Par la nature des régulations publiques, le projet politique qui les porte et la capacité d'action des pouvoirs en charge de leur mise en œuvre. Les politiques de formalisation observables dans les villes africaines reflètent la manière dont les pouvoirs politiques perçoivent leurs opportunités au regard des tensions engendrées par la transition urbaine et de la solidité de leurs alliances politiques : outil de réglage des relations entre formel et informel au sein des configurations de fourniture, elles sont un indicateur particulièrement signifiant de la manière dont les pouvoirs d'État entendent gérer et réguler le changement urbain.

\section{Conclusion}

La transition urbaine s'accompagne de profondes transformations sociales ainsi que d'une vive concurrence pour la consommation des espaces et des ressources. Un défi crucial des politiques urbaines est la régulation de processus contradictoires et l'invention de solutions permettant de les dépasser dans un contexte que dominent, depuis les années 1980, les politiques dites néolibérales. La formalisation de configurations de fourniture hybrides est une des manifestations de ce projet politique d'intégration par le marché, que nous avons qualifié de " tournant pragmatique ", lequel a un triple objectif du point de vue des pouvoirs d'État africains : accompagner la transition urbaine en accélérant la fourniture des services essentiels, satisfaire le désir de 
consommation des classes moyennes et atténuer leurs impatiences sociopolitiques, renégocier la fidélité des élites urbaines en étendant le domaine de leurs affaires.

Revenons à la question initiale : les villes africaines évoluent-elles vers des villes post-réseau ? Nous avons montré que le modèle du réseau existe au sein des configurations de fourniture et n'est pas remis en cause par leur formalisation : ré-encastré dans l'environnement matériel et social des villes, rendu plus fonctionnel par des dispositifs d'extension simplifiée ou des fonctionnalités hors réseau, il semble à la fois consolidé dans des périmètres limités et durablement concurrencé à l'extérieur. Dans ce contexte, la formalisation des configurations de fourniture des services essentiels est fondée, d'une part, sur des logiques d'empilement et d'hybridation entre des systèmes sociotechniques hétérogènes, d'autre part, sur une "normalisation " des pratiques marchandes chevauchant les catégories jusqu'alors distinctes du service public et des offres marchandes (en partie informelles), car l'enjeu, pour les pouvoirs publics, est à la fois de favoriser l'intégration par le marché et d'en contrôler les conditions.

Participant à la régulation politique de la transition urbaine, ces évolutions ne semblent pas, pour l'heure, contribuer à une transformation profonde des régimes sociotechniques urbains en Afrique. En important au cœur du système conventionnel de fourniture un principe d'hétérogénéité et une grammaire de la coordination entre une pluralité de dispositifs sociotechniques distincts, la formalisation des configurations de fourniture pourrait néanmoins fonder une troisième génération de réforme des services en réseaux, après la modernisation-décentralisation des opérateurs publics puis la privatisation des exploitants. Si le réseau n'est pas soluble dans le " tournant pragmatique ", son hégémonie l'est donc.

Cette perspective n'est pas sans conséquences et ses bénéfices, en termes d'extension de la fourniture des services et de soutien à l'émergence/consolidation des petites classes moyennes urbaines, ne doivent pas être sous-estimés. En termes généraux, toutefois, la contribution du "tournant pragmatique " à la production d'une ville " formalisée " plus équitable dans l'accès aux services collectifs et plus durable en termes de préservation environnementale reste une question ouverte. La reconnaissance des offres alternatives hors-réseaux ne dit rien, en effet, de l'identité des bénéficiaires, des marges et rentes qu'elles génèrent, de leurs éventuels effets d'éviction à l'encontre d'autres modes 
d'accès aux ressources, par exemple gratuits. Au fond, la formalisation des configurations de fourniture est moins une finalité qu'un outil transactionnel au service de la libéralisation du marché des services et de l'élargissement de l'offre à des petites classes moyennes que le modèle trop exclusif du réseau conventionnel avait jusqu'alors exclues : ni sa robustesse comme mode de régulation de l'hétérogénéité des sociétés citadines, ni sa capacité à assurer la percolation du progrès social au-delà des gagnants de la transition urbaine ne sont acquises. Elles devront être testées et évaluées dans la durée.

\section{Références}

Africa Progress Panel (2017), Lights, Power, Action. Electrifying Africa, s.l., APP.

Allen A. (2014), « Peri-urbanization and the political ecology of differential sustainability ", dans Parnell S., Oldfield S. (éds), A Routledge Handbook on Cities of the Global South, Londres, Routledge, p. 522-538.

Ahlers R., Schwartz K., Perez Guida V. (2013), "The myth of "healthy" competition in the water sector: the case of small scale water providers ", Habitat international, $\mathrm{n}^{\circ} 38$, p. 175-182.

Ahlers R., Cleaver F., Rusca M., Schwartz K. (2014), «Informal Space in the Urban Waterscape: Disaggregation and Co-Production of Water Services ", Water Alternatives, vol. 7, n 1, p. 1-14.

Andreasen M. H., Møller-Jensen L. (2016) « Beyond the networks: Selfhelp services and post-settlement network extensions in the periphery of Dar es Salaam ", Habitat international, n 53, p. 39-47.

Banerjee S., Morella E. (éds) (2011), Africa's Water and Sanitation Infrastructure: Access, Affordability, and Alternatives, Washington, World Bank.

Baron C., Bonnassieux A. (2013), «Gouvernance hybride, participation et accès à l'eau potable. Le cas des associations d'usagers de l'eau (AUE) au Burkina Faso ", Annales de géographie, n 693, p. 525-548.

Bédécarrats F., Lafuente-Sampietro O., Leménager M., Lukono Sow D. (2016), "Building commons to cope with chaotic urbanization? Performance and sustainability of decentralized water services in the outskirts of Kinshasa ", Journal of Hydrology, http://dx.doi.org/10.1016/j.jhydrol.2016.07.023. 
Berthélémy J.-C. (2016), « Les mini-réseaux électriques comme exemple d'application des thèses d'Elinor Ostrom sur la gouvernance polycentrique de la tragédie des communs ", communication pour la Conférence internationale de l'AFD sur le développement, 12 édition, Paris, 1-2 décembre 2016.

Berthélémy J.-C., Béguerie V. (2016), « Introduction. Decentralized electrification and development : initial assessment of recent projects ", Field Actions Science Reports, $2^{\mathrm{e}}$ semestre, p. 4-9.

Blanc A., Cavé J., Chaponnière E. (2009), Les petits opérateurs privés de la distribution d'eau à Maputo : d'un problème à une solution ?, Paris, AFD (Document de travail, $\mathrm{n}^{\circ} 85$ ).

Botton S., Blanc A. (2014), «Un service public marchand de proximité : l'action des petits opérateurs privés pour la desserte des quartiers périurbains en Afrique ", Actes de la recherche en sciences sociales, n²03, p. 106-113.

Buire C. (2014), "Suburbanisms in Africa? Spatial Growth and Social Transformation in New Urban Peripheries: Introduction to the Cluster ", African Studies, $\mathrm{n}^{\circ}$ 73, p. 241-244.

Bulkeley H., Castán Broto V., Maassen A. (2014), «Low-carbon Transitions and the reconfiguration of urban infrastructure ", Urban Studies, vol. 51, $\mathrm{n}^{\circ} 7$, p. 1471-1486.

Calas B. (2007), "Dynamiques métropolitaines d'Afrique orientale ", Les Cahiers d'Outre-Mer [en ligne], n 237, janvier-mars, doi:10.4000/ com.624

Chakava Y., Franceys R., Parker A. (2014), " Private boreholes for Nairobi'urban poor: the stop-gap or the solution? ", Habitat international, $\mathrm{n}^{\circ}$ 43, p. 108-116.

Chauveau J.-P., Le Pape M., Olivier de Sardan J.-P. (2001), « La pluralité des normes et leurs dynamiques en Afrique ", dans Winter G. (éd.), Inégalités et politiques publiques en Afrique, Paris, Karthala-IRD, p. 145-162.

Cheneau-Loquay, A. (2012), «La téléphonie mobile dans les villes africaines. Une adaptation réussie au contexte local ", L'Espace géographique, vol. 41, $\mathrm{n}^{\circ} 1$, p. 82-93. 
Coutard O., Rutherford J. (2013), "Vers l'essor de villes "post-réseaux” : infrastructures, innovation sociotechnique et transition urbaine en Europe ", dans Forest J., Hamdouch A. (éds), L'innovation face aux défis environnementaux de la ville contemporaine, Lausanne, Presses polytechniques universitaires romandes.

Criqui L. (2015), "Infrastructure urbanism: Roadmaps for servicing unplanned urbanisation in emerging cities ", Habitat international, vol. 47, p. 93-102.

Crombé L. (2017), «Enjeux d'échelles, enjeux politiques : l'approvisionnement et l'accès à l'eau dans les quartiers périphériques du Grand Khartoum (Soudan) ", Thèse de doctorat, Université Paris Nanterre.

Damon J. (2014), Le marché des classes moyennes dans les pays émergents : quelle réalité ? quelles opportunités?, Paris, CCI Île-de-France, coll. "Prospective et entreprise ", $\mathrm{n}^{\circ} 25$.

Darbon D. (2008), "Réformer un inexistant désiré ou supprimer un inopportun incontournable ? Le service public confronté à l'État et aux sociétés projetées en Afrique ", Téléscope (ENAP/Québec), hiver, p. $98-112$.

Darbon D. (2014), " Nom de code "Classes moyennes en Afrique". Les enjeux politiques d'une labellisation de groupes invisibles et vulnérables ", dans Darbon D., Toulabor C. (éds), Linvention des classes moyennes africaines : enjeux politiques d'une catégorie incertaine, Paris, Karthala, p. 15-59.

Darbon D., Toulabor C. (éds) (2014), Linvention des classes moyennes africaines : enjeux politiques d'une catégorie incertaine, Paris, Karthala.

Desjeux D. (2011), «Le chassé-croisé des classes moyennes mondiales ou la consommation comme analyseur de la nouvelle donne internationale ", http://www.argonautes.fr/2011-04-d-desjeux-classe-moyennemondiale-le-chasse-croise/

Dorier-Apprill E., Domingo E. (2004), «Les nouvelles échelles de l'urbain en Afrique ", Vingtième siècle. Revue d'histoire, vol. 81, n 1, p. 41-54.

Eberhard A., Rosnes, O., Shkaratan, M., Vennemo, H. (2011), Africa's power infrastructure: investment, integration, efficiency, Washington, World Bank. 
Foster V., Briceño-Garmendia C. (2010), Infrastructures africaines : une transformation impérative, Washington, Banque mondiale et AFD.

Franks L., Prasad G. (2014), Informal electricity re-selling: Entrepreneurship or exploitation?, Cape Town, University of Cape Town/Energy Research Centre.

Furlong K. (2014), "STS beyond the "modern infrastructure ideal": Extending theory by engaging with infrastructure challenges in the South ", Technology in Society, n 38, p. 139-147.

Gaunt T., Salida M., Macfarlane R., Maboda S., Reddy Y., Borchers M. (2012), Informal Electrification in South Africa: Experience, Opportunities and Challenges, Cape Town, Sustainable Energy Africa.

Ginisty K. (2014), «Inégalités et (in)justices spatiales à Maputo. Pratiques des services urbains ", Thèse de doctorat, Université Paris Nanterre.

Godard X. (éd.) (2002), Les transports et la ville en Afrique au sud du Sahara, Paris, Karthala-INRETS.

GSMA (2016), Mobile for Development Utilities. Unlocking access to utility services: The transformational value of mobile, Londres, GSMA, http:// www.gsma.com/mobilefordevelopment/wp-content/uploads/2016/07/ Mobile-for-Development-Utilities-Annual-Report.pdf.

Harre D., Gazel H., Moriconi-Ebrard F. (2015), « Les nouveaux territoires urbains d'Afrique de l'Est ", Territoire en mouvement. Revue de géographie et aménagement [en ligne], n 27-28, http://tem.revues.org/3100.

Hugon P. (2014), « L'informel ou la petite production marchande revisitée quarante ans après ", Mondes en développement, $\mathrm{n}^{\circ} 166, \mathrm{p} .17-30$.

Jaglin S. (1995), Gestion urbaine partagée à Ouagadougou : pouvoirs et périphéries (1983-1991), Paris, Karthala-ORSTOM.

Jaglin S. (2005), Services d'eau en Afrique subsaharienne: la fragmentation urbaine en question, Paris, CNRS Éditions.

Jaglin S. (2007), "Décentralisation et gouvernance de la diversité : les services urbains en Afrique anglophone ", dans Fourchard L. (éd.), Gouverner les villes d'Afrique : Etat, gouvernement local et acteurs privés, Paris, Karthala/CEAN, p. 21-34.

Jaglin S. (2008), « Differentiating networked services in Cape Town: echoes of splintering urbanism? ", Geoforum, vol. 39, n 6, p. 1897-1906. 
Jaglin S. (2012), "Services en réseaux et villes africaines : l'universalité par d'autres voies?", L'Espace géographique, $\mathrm{n}^{\circ}$ 1, p. 51-67.

Jaglin S. (2013), «Les dispositifs hors réseau des villes en développement : quels enseignements ? ", dans Deutsch J.-C., Gautheron I. (éds), Eau pour la ville, eaux des villes: Eugène Belgrand XIX'-XXI siècle, Paris, Presses des Ponts et Chaussées, p. 400-421.

Jaglin S. (2014), "Regulating Service Delivery in Southern Cities: Rethinking Urban Heterogeneity ", dans Parnell S., Oldfield S. (éds), $A$ Routledge Handbook on Cities of the Global South, Londres, Routledge, p. 434-447.

Jaglin S., Bousquet A. (2012), "Conflicts of influence and competing models: the boom in community-based privatization of water services in sub-Saharan Africa ", dans Barraqué B. (éd.), Urban water conflicts, Leiden, Taylor and Francis/UNESCO-IHP, p. 169-193.

Jaglin S., Zérah M.-H. (2010), «Introduction. Eau des villes : repenser des services en mutation ", Revue Tiers Monde, n² 203, p. 7-22.

Katusiimeh M., Burger K., Mol A. (2013), «Informal waste collection and its co-existence with the formal waste sector: the case of Kampala, Uganda ", Habitat international, $\mathrm{n}^{\circ} 38$, p. 1-9.

Kjellén M., McGranahan G. (2006), Informal Water Vendors and the Urban Poor, Londres, IIED (HSDP Series, Theme: Water-3).

Lall S. V., Vernon Henderson J., Venables A. J. (2017), Africa's Cities: Opening Doors to the World, Washington, World Bank.

Lautier B. (2004), L'économie informelle dans le tiers monde, Paris, La Découverte.

Lawaetz S., Smyser C. (2011), "Challenges and Opportunities in Electricity Service Provision for Urban BOP Communities ", dans Márquez P., Rufín C. (éds), Utilities at the Base of the Pyramid, Cheltenham (UK) and Northampton (Mass.), Edward Elgar Publishing, p. 134-156.

Lombard J. (2006), «Enjeux privés dans le transport public d'Abidjan et de Dakar », Géocarrefour, vol. 81, n 2, p. 167-174.

Lorrain D., Poupeau F. (2014), "Ce que font les protagonistes de l'eau. Une approche combinatoire d'un système sociotechnique ", Actes de la recherche en sciences sociales, $\mathrm{n}^{\circ} 203$, p. 4-15. 
Moretto L., Allen A. (2015), "Co-Producing Water Services in PeriUrban Caracas: Political Democratisation Without Administrative Decentralisation? ", dans Bogaert J., Halleux J.-M. (éds), Territoires périurbains : développement, enjeux et perspectives dans les pays du Sud, Gembloux, Presses agronomiques de Gembloux.

Myers G. (2015), «A World-Class City-Region? Envisioning the Nairobi of 2030 », American Behavioral Scientist, vol. 59, n³ 3, p. 328-346.

Nantchop V. (2017), " Politiques publiques de l'eau et gouvernance urbaine. Acteurs et enjeux de l'accès à l'eau potable des populations à Douala (Cameroun) », Thèse de doctorat, Université Paris 1 Panthéon Sorbonne.

Naulet F., Biteete L. (2014), Cahier $n^{\circ}$ 6. Quelle régulation pour les réseaux autonomes en RDC? Promouvoir l'extension des mini-réseaux et des bornesfontaines en RDC, Paris, GRET.

Naulet F., Gilquin C., Leyronas S. (2014), Eau potable et assainissement dans les villes $d u$ Sud: la difficile intégration des quartiers défavorisés aux politiques urbaines, Paris, GRET, coll. «Débats \& Controverses ", n 8.

Ngambi J. (2015), «Déchets solides ménagers dans la ville de Yaoundé (Cameroun) : de la gestion linéaire vers une économie circulaire », Thèse de doctorat, Université du Maine, Le Mans.

Olivier de Sardan J.-P. (2010), Local Governance and Public Goods in Niger, Londres, ODI (APPP working Paper, n 10).

Pierrat A. (2014), "Les lieux de l'ordure de Dakar et d'Addis Abeba. Territoires urbains et valorisation non institutionnelle des déchets dans deux capitales africaines ", Thèse de doctorat, Université Paris I Panthéon Sorbonne.

Pradeilles J.-C., Garcia-Oriol G., Tall I. (1991), L'organisation corporative des taxis collectifs à Bamako et Lomé : richesse et perspectives, Lyon, Codatu.

Rizzo M. (2011), "The Struggles of Informal Transport Workers in Tanzania: Debunking the Myth of Micro Solutions ", CDPR Development Viewpoint, $\mathrm{n}^{\circ} 68$.

Rutherford J., Coutard O. (2010), "Urban Energy Transitions: Places, Processes and Politics of Socio-technical Change ", Urban Studies, vol. 51, n 7 , p. 1353-1377. 
Schoon S., Altrock U. (2014), « Conceded informality. Scopes of informal urban restructuring in the Pearl River Delta ", Habitat international, $\mathrm{n}^{\circ} 43$, p. 214-220.

Sory I. (2013), " “Ouaga la belle !” Gestion des déchets solides à Ouagadougou : enjeux politiques, jeux d'acteurs et inégalités environnementales ", Thèse de doctorat, Université Paris I PanthéonSorbonne.

Star S., Ruhleder K. (2010), "Vers une écologie de l'infrastructure. Conception et accès aux grands espaces d'information ", Revue d'anthropologie des connaissances, vol. 4, $\mathrm{n}^{\circ} 1$, p. 114-161.

Trémolet S., Evans B. (2010), Output-Based Aid for Sustainable Sanitation, Washington, GPOBA/WSP (OBA Working Paper, $\mathrm{n}^{\circ} 10$ ).

Tsitsikalis A. (2011), Recyclage des déchets et développement durable. L'émergence d'une "filière sociale " de collecte et de traitement de déchets peut-elle contribuer au développement durable dans les villes du Sud?, Paris, GRET, coll. "Coopérer aujourd'hui ", n 74.

Valfrey-Visser B., Schaub-Jones D., Collignon B., Chaponnière E. (2006), Access through innovation: Expanding water service delivery through independent network providers. Considerations for practitioners and policymakers, Londres, BPD/AFD.

Van Dijk M. P., Etajak S., Mwalwega B., Ssempebwa J. (2014), « Financing sanition and cost recovery in the slums of Dar es Salaam and Kampala ", Habitat international, $\mathrm{n}^{\circ} 43$, p. 206-213.

Vliet B. van, Buuren J. van, Mgana S. (2014), Urban waste and sanitation services for sustainable development: harnessing social and technical diversity in East Africa, Londres, Routledge.

Water Alternatives (2014), Special issue: Informal Space in the Urban Waterscape, $n^{\circ} 7 / 1$.

Watson V. (2014), "African urban fantasies: dreams or nightmares? ", Environment and Urbanization, vol. 26, $\mathrm{n}^{\circ}$ 1, p. 215-231.

Wilkinson P. (2010), "Incorporating informal operations in public transport system transformation: the case of Cape Town, South Africa ", Brazilian Journal of Urban Management, vol. 2, $\mathrm{n}^{\circ}$ 1, p. 85-95. 


\title{
Infrastructures urbaines et transition bas-carbone
}

\section{Spatialiser les processus de diffusion d'innovations de niche : le cas des réseaux de chaleur en Alberta}

\author{
Aïda Nciri
}

Les infrastructures énergétiques ont une place importante dans les villes et participent aux émissions de gaz à effet de serre et au changement climatique. La transition bas-carbone nécessite donc une transformation des infrastructures énergétiques des villes afin de réduire leur empreinte carbone. Cette transformation peut passer par l'adaptation d'infrastructures existantes, ou bien la création et le déploiement d'infrastructures nouvelles, prenant la place des anciennes. C'est le cas des réseaux de chaleur en Alberta.

Les Transition Studies se sont établies ces dernières années comme un champ à part entière dans la littérature des systèmes sociotechniques. Les Transition Studies étudient les conditions qui permettent la diffusion d'innovations technologiques de niche et ayant la capacité de transformer les régimes sociotechniques existants. Bien que très diverses, leur socle commun repose sur : l'objectif d'éclairer les politiques publiques visant à mettre en œuvre une transition vers un développement soutenable ${ }^{1}$, l'utilisation de la perspective dite "multiniveaux $"^{2}$ comme cadre

$1 \quad$ Il ne s'agit pas d'un anglicisme. Parler de soutenabilité souligne la dimension de justice sociale et d'équité. En ce sens, un développement durable peut ne pas être soutenable pour tous. Pour une discussion approfondie, voir Vivien (2005).

2 Le cadre d'analyse de la perspective multiniveaux (Geels et Schot, 2007, 2010) conceptualise le processus de transition soutenable à travers l'imbrication de trois niveaux : la niche, le régime et le paysage. Ces niveaux représentent chacun un ordre social et technologique plus ou moins stabilisé et ne sont pas à confondre avec des échelles spatiales (Raven et al., 2012). La niche est le locus de l'innovation radicale 
d'analyse, et l'attention portée aux acteurs institutionnels plutôt qu'individuels (Chappin et Ligtvoet, 2014 ; Grin et al., 2010 ; Markard et al., 2012).

Néanmoins, les Transition Studies ont délaissé les infrastructures énergétiques urbaines comme objet d'études, privilégiant les innovations, technologies détachées de leurs propriétés infrastructurelles. L'infrastructuralisation des Transition Studies permettrait de répondre à deux séries de critiques qui leur ont été formulées. Une première série se concentre sur l'absence de conceptualisation de l'espace (Coenen et Truffer, 2012 ; Raven et al., 2012 ; Truffer et Coenen, 2012). Cette absence de dimension spatiale se retrouve dans la réification des niveaux de gouvernement, et le biais qui consiste à centrer l'analyse sur l'échelle nationale, au détriment des autres échelles (régionales, municipales...) et des rapports dynamiques qu'ils entretiennent. De ce fait, l'espace urbain est ignoré et les quelques exceptions tendent à considérer ces espaces comme des réceptacles homogènes et inertes de la transition. En outre, l'absence de considérations géographiques ne permet pas de prendre en compte les variations et déséquilibres territoriaux qui peuvent freiner ou favoriser la diffusion d'une innovation (Dodson, 2013). Les infrastructures de réseaux sont des objets géographiques : elles ont une empreinte spatiale et un ancrage territorial qui conditionnent leur équilibre économique et leur caractéristique matérielle. Étudier les conditions de diffusion de nouvelles infrastructures de réseaux permet de spatialiser les Transition Studies.

Une deuxième série de critiques que l'auteure adresse aux Transition Studies concerne leur méthodologie et épistémologie. Les Transition Studies cherchent à identifier les politiques publiques qui créent les conditions favorables à la diffusion d'une technologie de niche dans un régime sociotechnique donné. Néanmoins, la compréhension de ces processus pourrait être enrichie par une attention portée aux échecs de

qui cherche à être diffusée et à transformer l'ensemble du système. Le niveau d'instabilité y est élevé, car les règles et les interactions sociotechniques sont en train d'être constituées et testées par les acteurs qui souhaitent diffuser l'innovation. Le régime représente un niveau plus stabilisé où les règles et relations sociales sont établies autour d'un système sociotechnique dominant. Le paysage représente la longue durée, les forces structurantes d'un ordre social et technologique très stable, qui se transforment sur le long terme et sur lequel les agents ont peu de prise. 
diffusion - n'apprend-on pas de ses échecs ? Nous considérons que cette attention aux échecs invite à compléter l'approche systémique et centrée sur des politiques publiques offerte par les Transition Studies, avec une approche par projet. Dans ce cas, la diffusion d'une technologie, ou dans notre cas d'une infrastructure, passe par une réplication de ces projets. Cette approche élargit l'analyse aux stratégies déployées par des acteursagents - et non plus seulement par des acteurs institutionnels - et permet de comprendre comment l'imbrication entre structure et individu participe au succès, ou aux échecs, d'un projet à sa réplication ou non.

Les réseaux de chaleur urbains ${ }^{3}$ peuvent être considérés comme une infrastructure de niche au Canada, et notamment dans la province de l'Alberta (CIEEDAC, 2014). Les réseaux de chaleur sont un système de chauffage décentralisé - c'est-à-dire non relié au réseau de gaz naturel, décentralisée produisant de la chaleur centralisée à partir d'une ou plusieurs chaufferies, distribuant cette chaleur sous forme d'eau chaude ou de vapeur à plusieurs bâtiments et/ou industries à travers un réseau de canalisations souterraines.

Le présent chapitre propose de pallier la double critique faite aux Transition Studies en étudiant deux projets de réseaux de chaleur en Alberta, et en tentant de comprendre pourquoi l'un des deux projets est sur le point d'être répliqué, et pas l'autre. Premièrement, cette approche de la transition par une infrastructure de réseaux décentralisée permet d'urbaniser les Transition Studies en montrant l'importance de l'aménagement urbain dans le processus de diffusion d'une innovation. Deuxièmement, cet article invite les Transition Studies à mobiliser les concepts géographiques. En intégrant les stratégies spatiales déployées par les acteurs au vu des contraintes infrastructurelles des réseaux de chaleur, l'article démontre comment ces stratégies participent à la réplication (ou non) de projets et, sur le moyen terme à la diffusion d'innovation.

Nous commençons par présenter les deux projets de réseaux de chaleur (I) avant de comprendre le contexte géographique et énergétique dans lequel ils ont été développés et mis en service (II). Puis nous nous intéressons aux stratégies spatiales déployées par les porteurs de projets

Les réseaux de chaleur sont utilisés par l'industrie pétrolière sur les sites d'extraction de schistes bitumineux, ainsi que sur le patrimoine d'institutions publiques comme les hôpitaux et les universités. 
et comment la prise en compte de l'espace et des enjeux du territoire a permis au projet de Calgary d'atteindre un équilibre économique qui est en passe d'être répliqué alors que le projet d'Okotoks est toujours un projet de démonstration confronté à des difficultés économiques (III). Enfin, nous comparerons les bénéfices environnementaux des projets pour remarquer que cette analyse est mouvante et dépend de l'échelle spatio-temporelle que l'on adopte et conclurons sur l'importance de la transformation de l'espace urbain dans la mise en œuvre de la transition bas-carbone.

\section{Deux projets de réseaux de chaleur}

Les projets de Calgary et d'Okotoks sont deux projets de réseaux de chaleur avec des caractéristiques différentes : ils utilisent des technologies de production de chaleur différente (cogénération vs solaire thermique), ont été développés dans des espaces urbains opposés (programme de redéveloppement dense vs nouveau programme pavillonnaire), ont été portés par des différents acteurs (municipalité et énergéticien local vs agence du gouvernement fédéral) et ont des objectifs distincts (entreprise commerciale $v s$ démonstration technologique).

\section{Calgary, un réseau de chaleur commercial}

La ville de Calgary est la capitale économique de la Province de l'Alberta. Avec près de 1,2 million d'habitants, c'est la plus grosse ville de la province. Son organisation spatiale suit l'archétype des grandes villes nord-américaines : le centre d'affaires (District Business Centre) concentre les activités économiques et les tours de bureaux dans un bâti dense, tandis que les suburbs, ces zones pavillonnaires nord-américaines avec maisons individuelles et jardinet, s'étalent tout autour.

Le projet de réseau de chaleur urbain a été initié au début des années 2000 par la ville de Calgary et la compagnie d'électricité Enmax. Enmax est née de la privatisation des services énergétiques de la ville de Calgary en 1998, et son capital est entièrement détenu par elle. De ce fait, une porosité institutionnelle existe entre les deux organisations (les conseillers municipaux siègent au sein du comité de direction de la compagnie), bien qu'Enmax soit soumise aux règles du marché. 
Le projet a été développé en même temps qu'un ambitieux programme de reconversion d'une ancienne friche industrielle en zone résidentielle avec tours d'habitations. Ce programme d'aménagement municipal, appelé East Village, se situe à proximité du centre d'affaires. Sa mise en œuvre est sous la responsabilité d'une entité commerciale détenue par la ville, le Calgary Municipal Land Corporation. Enmax a souhaité développer ce projet de réseau de chaleur en lien avec ce projet urbain afin de profiter de la densité du bâti, qui garantissait un débouché commercial au réseau de chaleur. Cela lui permettait également de profiter des synergies sur les travaux de voiries.

Le projet comprenait deux phases : le développement du réseau structurant de distribution à East Village et la construction d'une chaufferie au gaz naturel, puis la construction d'une centrale à cogénération au gaz naturel avec une capacité électrique de $165 \mathrm{MW}$. Le réseau structurant et la chaufferie d'appoint ont été construits et mis en service en 2010. Le projet a bénéficié d'une subvention de 30 millions de dollars canadiens de la part du gouvernement fédéral, du gouvernement provincial et de la municipalité de Calgary. Enmax a également déboursé un montant estimé entre 10 et 20 millions de dollars canadiens.

Néanmoins, le succès commercial d'Enmax n'a pas été au rendezvous. La ville de Calgary a refusé de classer le réseau (c'est-à-dire rendre la connexion obligatoire pour tout nouveau bâtiment) dans la zone d'East Village et Enmax a eu des difficultés à convaincre les promoteurs à se raccorder du fait de la nouveauté des réseaux de chaleur urbains dans la province, de l'impréparation commerciale de la compagnie, ainsi que de la forte diminution des prix du gaz. La construction de la centrale à cogénération a été suspendue en raison du faible niveau de demande de chaleur et des faibles prix de l'électricité sur le marché provincial qui ne permettaient pas un retour sur investissement suffisant. Durant les deux premières années de mise en service, l'Hôtel de Ville a été le seul bâtiment raccordé au réseau.

Néanmoins, à partir de 2012, une série de facteurs a permis à Enmax de raccorder ses premiers clients : changement de l'équipe commerciale menant à revoir la stratégie de développement (tarifs attractifs pour les premiers clients raccordés), rapprochement avec Calgary Municipal Land Corporation, l'entité municipale en charge 
du développement d'East Village, afin de promouvoir le système aux promoteurs immobiliers, et présentation du réseau de chaleur urbain lors de différents événements institutionnels liés à l'aménagement urbain. En quelques années, le nombre d'abonnés aux réseaux s'est considérable accru (tableau 1).

Tableau 1. Croissance du réseau de chaleur de Calgary, nombre de bâtiments connectés

\begin{tabular}{|l|c|c|}
\hline & Nouveaux bâtiments & Bâtiments cumulés \\
\hline 2010 & 1 (City Hall) & 1 \\
\hline 2011 & 0 & 1 \\
\hline 2012 & 3 & 4 \\
\hline 2013 & 0 (inondations) & 4 \\
\hline 2014 & 9 & 13 \\
\hline 2015 & 4 & 17 \\
\hline 2016 (prévision) & 9 & 26 \\
\hline
\end{tabular}

Source : d'après Enmax, Présentation à l'Université de Calgary, 12 février 2015.

Deux réplications du projet de réseau de chaleur, avec une petite centrale de cogénération au gaz, ont été envisagées dans le cadre de nouveaux programmes d'aménagement à Calgary. Le programme West Campus, situé aux abords de l'université, a finalement abandonné le projet de réseau de chaleur en 2015, car jugé non rentable du fait du faible prix de l'énergie dans la province, et du calendrier de développement du programme - s'étalant sur une décennie, le réseau n'aurait pas été amorti suffisamment rapidement. Par contre, le projet West Village (le pendant d'East Village), développé également par la municipalité, a validé la construction d'un nouveau réseau de chaleur urbain pour alimenter le futur quartier qui devrait voir le jour dans les prochaines années.

\section{Drake Landing Solar Community (DLSC), un réseau de chaleur de démonstration technologique}

DLSC est un projet visant à démontrer la faisabilité du stockage saisonnier de chaleur solaire en sous-sol. Il a été porté et financé par une agence de Recherche et Développement rattachée au ministère 
fédéral en charge de l'énergie (Ressources Naturelles Canada, RNCAN) et achevé en 2007. Le partenariat autour de ce projet entre le directeur de l'agence (et également directeur du projet), la ville d'Okotoks et des développeurs s'est fait à travers un concours de circonstances.

Achevé en 2007, le système a été dimensionné pour approvisionner un nouveau lotissement pavillonnaire de 52 maisons labélisées " R-2000 ", c'est-à-dire avec des performances énergétiques $30 \%$ supérieures aux standards en vigueur. Des panneaux solaires thermiques posés sur le toit de chacune des maisons captent la chaleur du soleil pour chauffer un premier circuit d'eau relié à un échangeur thermique. Cet échangeur thermique chauffe ensuite un deuxième circuit d'eau souterrain qui réchauffe à son tour le sous-sol. À la fin de l'été, la température du sous-sol peut atteindre 80 degrés Celsius (DLSC, 2015). En hiver, la chaleur emmagasinée par le sous-sol est récupérée à travers l'échangeur thermique qui chauffe un troisième circuit relié aux maisons. Depuis sa mise en service, plus de $90 \%$ des besoins de chaleur de chauffage des maisons sont fournis par le système.

L'eau chaude sanitaire est fournie à hauteur de $40 \%$ par des capteurs solaires thermiques individuels et indépendants du premier système. Enfin, toutes les maisons sont raccordées au réseau de gaz naturel afin de fournir le complément de chaleur pour le chauffage $(10 \%)$ et pour l'eau chaude sanitaire $(60 \%)$.

Ce projet de démonstration a coûté 7 millions de dollars canadiens et près de $90 \%$ de ce montant a été financé par des subventions publiques venant des gouvernements fédéral et provincial. 
Tableau 2. La répartition du coût des éléments du projet de Drake Landing Solar Community, par maison

\section{Coût total du projet : 7 millions de dollars canadiens (\$CAD)}

Coût par maison : 134000 \$CAD

Poste des coûts

- Coûts liés à l'isolation des maisons pour atteindre le label R-2000 : 6400 \$CAD par maison

- Système de collecteur solaire : 14800 \$CAD par maison

- Adaptation des toitures des garages : 4000 \$CAD par maison

- Système de stockage solaire de court terme : 6000 \$CAD par maison

- Canalisation du réseau (hors stockage) : 6000 \$CAD par maison

- Système de stockage sous-terrain de chaleur thermale : 12000 \$CAD par maison

- Équipement supplémentaire : 50000 \$CAD par maison

- Design et modélisation : -3500 000 \$CAD par maison

- Contingences liées à la construction : - 1 000000 \$CAD par maison

Source : Howell-Mayhew Engineering, Présentation Powerpoint, 21 juillet 2008, http:// www.hme.calpresentations/ (consulté le 10 septembre 2016).

Les objectifs de démonstration technologiques du projet ont été atteints : le système contribue à plus de $90 \%$ aux besoins de chauffage des 52 maisons et permet la réduction de 5 tonnes d'émission de carbone par maison et par an, soit 260 tonnes par an. Il faut souligner qu'environ deux cinquièmes des tonnes de carbone évitées sont dues aux performances énergétiques des maisons.

Depuis 2010 et la fin de la période de test, le directeur de l'agence et du projet a tenté de reproduire ce projet à plus grande échelle dans d'autres villes canadiennes, et même à Okotoks. Néanmoins, plus de sept ans après, et malgré les nombreux prix et récompenses reçus par le projet, celui-ci n'a pas été répliqué et en est toujours au stade de démonstration. 


\section{Deux projets dans un contexte urbain hostile et un contexte énergétique temporairement favorable}

La construction simultanée, mais indépendante de ces deux réseaux de chaleur s'est produite dans un espace urbain hostile aux réseaux de chaleur et dominé à $98 \%$ par les chaudières individuelles ou collectives à gaz (NRCAN, 2012). Ces deux projets ont pu voir le jour grâce aux subventions de l'État fédéral, aux infrastructures et énergies vertes versées dans le cadre de politiques publiques naissantes sur le changement climatique, ainsi qu'aux prix élevés de l'énergie. Néanmoins, cette conjoncture favorable s'est interrompue en 2007-2008, avec l'arrivée d'un gouvernement fédéral conservateur au pouvoir au niveau et la chute des prix de l'énergie en Amérique du Nord.

\section{Réseaux de chaleur et aménagement urbain : les suburbs albertaines, un espace urbain hostile}

Les réseaux de chaleur sont une infrastructure qui produit de la chaleur centralisée à partir d'une ou plusieurs chaufferies, et qui distribue cette chaleur sous forme d'eau chaude ou de vapeur à plusieurs bâtiments et/ou industries à travers un réseau de canalisations souterraines. Les chaufferies peuvent être alimentées par différentes sources d'énergie (énergie fossile, biomasse, géothermie ou solaire thermique) ou bien récupérer la chaleur fatale de procédés industriels, comme dans le cas de la cogénération par exemple. Ils peuvent couvrir différentes échelles, et couvrir quelques hectares reliant un petit nombre de bâtiments dans un quartier, ou bien plusieurs dizaines de kilomètres carrés et dimensionnés à l'échelle d'une ville entière.

Léquilibre économique des réseaux de chaleur dépend de l'espace urbain dans lequel ils sont développés, de la demande en chaleur des bâtiments, et du prix de l'énergie. Les réseaux de chaleur urbains nécessitent des investissements considérables et un minimum de densité énergétique doit être assuré afin de garantir un volume minimal de vente de chaleur permettant un retour sur investissement généralement sur 20 à 30 ans. La densité énergétique est dépendante de la densité du bâti ainsi que de sa demande en chaleur. 
Par conséquent, le développement de réseaux de chaleur est étroitement lié à l'aménagement urbain. Ces infrastructures lourdes doivent être pensées en relation avec la fabrique urbaine, à la fois d'un point de vue opérationnel (intégration des canalisations dans le sous-sol, emplacement de la chaufferie...) et d'un point de vue de planification urbaine (densité du bâti, fonction urbaine...).

Un des écueils auxquels les réseaux de chaleur sont confrontés en Alberta est la faible densité des zones urbaines. En 2011, l'Alberta comptait 3,7 millions d'habitants, dont $83 \%$ vivant dans des zones urbaines (Statistique Canada, 2011a). Deux tiers de la population se concentrent dans les villes de Calgary et d'Edmonton (Statistique Canada, 2011b). Ces deux régions urbaines ont connu une forte croissance de leur population du fait de l'activité économique du secteur pétrolier et gazier (Statistique Canada, 2011b).

Cette croissance urbaine a été principalement absorbée à travers un étalement urbain et le développement de suburbs, ces lotissements pavillonnaires nord-américains constitués de maisons individuelles avec jardin. Par exemple, $94 \%$ de la croissance de population de la ville de Calgary a été absorbée par la construction de nouvelles suburbs aux limites de la ville, ce qui a favorisé l'étalement urbain et repoussé les limites de la ville (tableau 3). La ville compte aujourd'hui 1,2 million d'habitants pour une superficie de 825 kilomètres carrés, soit une densité moyenne de $1329 \mathrm{hab} / \mathrm{km}^{2}$ (Statistic Canada, 2011c) ${ }^{4}$. Les prévisions prévoient une augmentation de la population de 1,3 million dans les 50-60 années à venir.

La ville d'Okotoks, en tant que ville satellite résidentielle de la ville de Calgary, a suivi la même trajectoire de façon encore plus spectaculaire. Entre 2006 et 2011, la population d'Okotoks a augmenté de $42 \%$ pour atteindre 25000 habitants (Statistiques Canada, 2011d, Recensement de la population). La ville est dominée par les suburbs ( $77 \%$ de maisons individuelles séparées) et sa densité moyenne est de 1 273,8 habitants par $\mathrm{km}^{2}$ (tableau 4).

4 Pour comparaison, la superficie de Paris est huit fois plus petite pour une densité de $21234 \mathrm{hab} / \mathrm{km} 2$ (INSEE, 2011). 
Tableau 3. Croissance de la population et de la superficie de Calgary

\begin{tabular}{|c|c|c|c|c|}
\hline Année & Population & $\begin{array}{c}\text { Croissance } \\
\text { démographique }\end{array}$ & $\begin{array}{c}\text { Superficie } \\
\left(\mathrm{km}^{2}\right)\end{array}$ & $\begin{array}{c}\text { Densité } \\
\left(\text { habitant/ } \mathbf{k m}^{2}\right)\end{array}$ \\
\hline 1996 & 768082 & - & - & \\
\hline 2001 & 878866 & $14.4 \%$ & 601,79 & 1,460 \\
\hline 2006 & 988812 & $12.4 \%$ & 626,50 & 1,578 \\
\hline 2011 & 1096833 & $10.9 \%$ & 725 & 1,511 \\
\hline
\end{tabular}

Note : La superficie ne tient pas compte des $100 \mathrm{~km}^{2}$ de parcs et d'espaces verts de la ville Source : Statistique Canada, Recensement de la population de 1996, 2001, 2006 et 2011.

Tableau 4. Croissance de la population et de la superficie d'Okotoks

\begin{tabular}{|l|c|c|c|c|}
\hline Année & Population & $\begin{array}{c}\text { Croissance } \\
\text { démographique }\end{array}$ & $\begin{array}{c}\text { Superficie } \\
\left.\mathbf{( k m}^{2}\right)\end{array}$ & $\begin{array}{c}\text { Densité } \\
(\mathbf{h a b i t a n t /} \\
\left.\mathbf{k m}^{2}\right)\end{array}$ \\
\hline 1996 & 8,528 & - & - & \\
\hline 2001 & 11689 & $36,8 \%$ & 17,91 & 651,3 \\
\hline 2006 & 17150 & $46,7 \%$ & 18,55 & 924,4 \\
\hline 2011 & 24511 & $42,9 \%$ & 19,24 & 1273,8 \\
\hline $\begin{array}{l}\text { Note : Laugmentation de la densité s'explique par le développement de nouveaux } \\
\text { lotissements sur des zones encore non développées, mais déjà intégrées au territoire } \\
\text { de la municipalité }\end{array}$
\end{tabular}

Source : Statistique Canada, Recensement de la population de 1996, 2001, 2006 et 2011.

\section{Réseaux de chaleur et système énergétique : instabilité des politiques publiques et volatilité des prix}

Sur le front énergétique, les deux projets de réseaux de chaleur ont été développés dans un contexte doublement favorable, avec d'une part un début de prise en compte du changement climatique et de la réduction des gaz à effet de serre dans les politiques publiques fédérales et provinciales, et d'autre part des prix élevés de l'énergie, notamment du gaz naturel.

La prise en compte du changement climatique au niveau fédéral s'est traduite par la ratification du protocole de Kyoto en 1997 et l'adoption d'une série de mesures en 2002 et 2005 demandant au secteur industriel du pays de réduire leurs émissions de carbone et mettant en place une 
série de fonds verts pour financer des projets environnementaux. Au niveau provincial, le gouvernement de l'Alberta s'était également engagé à réduire ses émissions de GES avec l'adoption d'un plan climat en 2002 et la mise en place d'une taxe carbone entrée en vigueur en 2008.

Dans ce contexte, les deux projets de réseaux de chaleur se présentaient comme une alternative compétitive à un système énergétique carboné et dont les prix avaient connus une hausse continue depuis le début des années 2000. Les subventions publiques ont levé le principal obstacle à la mise en ouvre des projets : des investissements initiaux lourds et difficilement amortissables.

Néanmoins, ce contexte doublement favorable a été de courte durée. L'arrivée au pouvoir des conservateurs au niveau fédéral aux élections de 2006 a donné un coup de frein aux politiques publiques sur le changement climatique qui se mettaient en place. En 2011, le gouvernement fédéral se retirait du protocole de Kyoto. En parallèle, le gouvernement a mis en place une politique centrée sur l'extraction des énergies fossiles, dont l'Alberta concentre les ressources. Au niveau provincial, le prix et le périmètre de la taxe carbone se sont avérés très laxistes (Read, 2014) et sans effet sur l'industrie pétrolière qui représentait environ $25 \%$ du PIB de la province. En outre, les prix du gaz naturel se sont écroulés dans la seconde moitié des années 2000, rendant les énergies renouvelables et la cogénération non économiques. C'est dans ce contexte défavorable que les deux réseaux de chaleur ont été mis en service, et c'est ce qui explique que la centrale à cogénération n’a pas été construite.

\section{Une capacité de réplication liée aux stratégies spatiales déployées par les acteurs et à l'ancrage territorial du projet}

Le projet de DLSC peut être considéré comme un succès technique : le système fournit plus de $90 \%$ de chaleur solaire pour le chauffage des maisons raccordées. Au contraire, le projet de Calgary est un projet techniquement contrarié puisque la centrale de cogénération, élément clé de la production de chaleur verte, n’a pas été construite. Néanmoins, sur le moyen terme, et du point de vue de la capacité de réplication de 
projet de réseau de chaleur urbain, le projet de Calgary a réussi à faire un émule, alors que le projet de DLSC, malgré son succès technique, ne suscite aucun intérêt. Les raisons qui expliquent cette (non-)volonté de réplication sont à trouver dans la capacité des porteurs de projet à démontrer (ou non) la rentabilité commerciale des réseaux de chaleur dans les zones urbaines albertaines, ainsi que dans leur capacité à construire un projet de territoire et des alliances afin de développer un projet d'aménagement urbain compatible avec les critères de densité des réseaux de chaleur.

\section{Construire un équilibre économique : stratégies spatiales et espace urbain}

Dès la conception du projet, Enmax a stratégiquement pris en compte les caractéristiques territoriales de la ville de Calgary de façon à assurer un équilibre économique au projet. La première stratégie territoriale est la prise en compte de l'hétérogénéité de l'espace urbain, en termes de densité et de fonction. Enmax a catégorisél'espace urbain et privilégié les zones denses (centre d'affaires et East Village) aux zones peu denses (suburbs). Il a également réussi à équilibrer les fonctions urbaines s'offrant la possibilité de raccorder des zones commerciales (centre d'affaires) et résidentielles (East Village). Cette mixité de fonction est importante pour optimiser les moyens de production de chaleur et éviter des pics de demande trop élevés. Cette discrimination spatiale, malgré un début difficile, a permis à Enmax de démontrer que certaines zones des villes albertaines sont propices aux réseaux de chaleur.

La deuxième stratégie territoriale est celle d'une extension territoriale du réseau. L'emprise spatiale du réseau d'Enmax est malléable : le territoire desservi peut s'étendre afin de raccorder de nouveaux bâtiments en fonction des opportunités commerciales et des possibilités de construction de nouvelles chaufferies. Suite à un long travail de terrain, Enmax a cartographié le calendrier de remplacement des chaudières collectives des bâtiments présents dans la zone de desserte du réseau. Cette cartographie vise à démarcher de futurs clients suffisamment en amont afin de les encourager à se raccorder au réseau extensible de chaleur plutôt que d'engager les investissements pour remplacer les chaudières 
afin qu'ils se raccordent au réseau. Cette appropriation commerciale $d u$ territoire urbain a permis au projet d'optimiser les raccordements au sein de la zone de desserte et de raccorder des bâtiments anciens.

Ce rapport de prédation au territoire urbain a donc été déterminant pour la mise en place d'une offre commerciale aussi compétitive que le régime de chaudières au gaz et construire un modèle économique ciblé sur les zones urbaines denses, au point que d'autres programmes ont envisagé un réseau de chaleur, et dans le cas de West Village l'ont même adopté.

Au contraire, le réseau de chaleur de DLSC a ignoré la dimension territoriale et a conçu un réseau de chaleur insulaire et clos. Celui-ci a été dimensionné à l'échelle du lotissement des 52 maisons : aucune nouvelle maison ne peut être raccordée au système et la production de chaleur est inextensible. Paradoxalement, cette insularité du réseau de chaleur n’a pas été accompagnée par une autonomie énergétique. Plutôt que de construire et de raccorder une petite chaufferie d'appoint alimentant le réseau de chaleur, une dépendance territoriale au système de chauffage dominant a été créée : les maisons ont été raccordées au réseau de gaz naturel afin de fournir un complément de chauffage. Cette dépendance matérielle vis-à-vis du régime dominant a entraîné une redondance dans les réseaux de chauffage qui s'est traduite par un surcoût pour les ménages. Ces derniers paient à la fois les frais d'entretien du réseau de chaleur ainsi que les frais d'abonnement au réseau de gaz. En outre, le projet a été développé sur un territoire avec une faible densité énergétique (zones pavillonnaires) rendant le coût du projet exorbitant. Cette faible densité ne permet pas non plus au projet de générer suffisamment de revenus pour couvrir les coûts d'entretien du système.

Insularité du réseau de chaleur, dépendance matérielle au régime sociotechnique dominant et intégration dans un aménagement urbain pavillonnaire avec une faible densité énergétique sont autant d'incohérences spatiales de conception qui n'ont pas permis le développement d'un modèle économique. Ces incohérences ont même fini par attirer l'attention sur les coûts exorbitants de la technologie de stockage saisonnier d'énergie thermique solaire en comparaison de ses bénéfices environnementaux. Le projet est donc resté figé dans une symbolique de prouesse technologique coûteuse, sans intérêt commercial. 


\section{Ancrer le projet sur un territoire : projet de territoire et réseaux d'acteurs}

Les porteurs du projet du réseau de chaleur de Calgary ont réussi à ancrer le projet et l'infrastructure des réseaux de chaleur dans un projet territorial plus large de ville compacte et de densification urbaine. L'étalement urbain comme mode d'absorption de la croissance de la population de la ville pose d'importants problèmes financiers et budgétaires à la municipalité. Les coûts croissants d'opération et de maintenance des infrastructures et services urbains viennent grever durablement le budget de la municipalité. Le département des services financiers de la ville projette un déficit cumulé de 7 milliards d'euros lié aux dépenses d'entretien et de maintenance des infrastructures municipales dans les dix prochaines années (City of Calgary, 2011b).

Depuis le milieu des années 2000, la ville a tenté de mettre en œuvre des politiques d'aménagement pour freiner l'étalement urbain, notamment en densifiant les zones déjà aménagées, en encourageant la mixité des usages et en développant les transports en commun. Le plan municipal d'aménagement urbain (Municipal Development Plan) adopté en 2009 se fixe pour objectif d'absorber près de $30 \%$ de la croissance de la population dans les zones urbaines déjà développées d'ici 2039, et près de $70 \%$ d'ici 2069. Ces pourcentages sont à comparer aux $6 \%$ de l'année 2009. Les projets d'East Village et de West Village s'inscrivent d'ailleurs dans cette stratégie.

Les caractéristiques spatiales de forte densité nécessaire à l'équilibre économique des réseaux de chaleur sont en phase avec les objectifs de densification de la ville de Calgary. C'est pourquoi le département d'aménagement urbain de la ville a décidé d'utiliser les réseaux de chaleur comme un indicateur de densification dans le Plan de Développement Urbain. Ainsi, le Plan indique que « la part du territoire municipal ayant une densité suffisante pour accueillir un projet de réseau de chaleur [doit passer] de 0,3\% en 2005 à 1,7 \% d'ici 60 ans " (City of Calgary, 2009, section 5-10). La "densité suffisante " a été précisée dans un rapport d'évaluation du suivi des objectifs du MDP : "Pour qu'un réseau de chaleur soit viable, une densité minimum de 30 habitations 
(ou 100 personnes ou emplois) par hectare est nécessaire $"^{5}$ (City of Calgary, 2013, p. 40). L'unité utilisée, liée au bâti, et non pas aux consommations d'énergie, confirme le lien étroit fait par la ville entre réseau de chaleur et objectif de densification.

Les réseaux de chaleur deviennent même un élément à part entière de l'aménagement urbain et un outil pour accompagner la densification de Calgary. La ville a demandé aux aménageurs de considérer les réseaux de chaleur dans certains programmes urbains. Par exemple, deux plans d'aménagement urbains (area structure plan) ${ }^{6}$ ont obligé des aménageurs à mener une étude de faisabilité sur la viabilité d'un microréseau de chaleur, et ce afin d'encourager les développeurs à densifier les programmes.

En outre, le système devait participer au Plan de Réduction des Gaz à Effet de Serre élaboré par la ville de Calgary à l'époque du projet, et adopté en 2011. Ce plan fixe un objectif de réduction d'émissions de carbone de $20 \%$ d'ici 2020 et de $80 \%$ d'ici 2050 par rapport à 2005 (City of Calgary, 2011a) et considère les réseaux de chaleur comme un outil majeur de réduction des émissions de la ville.

Le réseau de DLSC, quant à lui, a été développé dans le cadre d'une zone pavillonnaire. Au contraire de Calgary, l'étalement urbain d'Okotoks est toujours source de croissance économique pour la municipalité, car il apporte de nouvelles ressources fiscales et crée des emplois. Ce modèle d'aménagement urbain a d'ailleurs été réaffirmé dans les deux plans successifs d'aménagement de la ville en 2012 et 2016. Le projet de réseau de chaleur de capture solaire thermique à l'échelle de 52 maisons ne remettait donc pas en cause le modèle de développement pavillonnaire adopté par la ville. Paradoxalement, cet alignement de circonstances autour d'une zone d'aménagement pavillonnaire a permis l'émergence d'un projet de démonstration, mais a également freiné sa réplication en pointant l'incompatibilité économique entre faible densité et réseau de chaleur, démontrant ainsi l'incompatibilité entre deux projets territoriaux : celui de l'aménagement urbain de la Ville d'Okotoks et celui de l'infrastructure de réseau de

5 «For district energy systems to be viable, a minimum density of 30 dwelling units (or 100 people and jobs) per hectare is required. "

6 Document déterminant le cadre général de nouveaux développements urbains (indication de l'usage des sols, de la densité, des transports...). 
chaleur. En outre, le plan de développement soutenable de la ville ne fixe aucun objectif de réduction des émissions de gaz à effet de serre (Town of Okotoks, 2016).

Deuxièmement, l'ancrage territorial différentié des deux projets est également lié à la capacité des porteurs de projets respectifs de former des alliances, ou réseaux, avec les acteurs du territoire. Le projet de DLSC a été entièrement porté par le directeur d'une agence fédérale qui n'a pas associé financièrement, politiquement et techniquement les acteurs locaux, notamment la ville d'Okotoks, l'énergéticien en charge de l'opération et la maintenance du système et le promoteur. La conceptualisation et les modélisations poussées du système de stockage ont été faites par des experts internationaux et le financement a été presque intégralement porté par des acteurs extérieurs au territoire d'Okotoks. En apportant un projet clé en main directement du niveau fédéral au niveau local, sans impliquer les acteurs locaux, le projet a constitué un réseau d'acteurs local mou. Les acteurs locaux ne se sont pas approprié l'infrastructure et sa technologie. À l'inverse, le projet de Calgary a impliqué trois acteurs du territoire : la municipalité, l'énergéticien Enmax qui appartient à la municipalité, et l'aménageur municipal Calgary Municipal Land Corporation. Malgré les tensions qui ont pu exister entre les trois acteurs (notamment autour du classement du réseau), la préexistence de ces liens a permis de développer le projet (subvention de 10 millions de dollars canadiens de la part de la ville de Calgary) et de contribuer à son succès économique avec le partenariat entre l'aménageur et l'énergéticien pour convaincre les promoteurs de se connecter. L'existence de liens entre les trois acteurs a été renforcée pendant le projet, contribuant à développer un réseau d'acteurs local fort.

La vision d'un projet territorial compatible entre aménagement urbain, transition énergétique et réseaux de chaleur ainsi que les liens étroits entre acteurs urbains et énergéticiens au niveau municipal expliquent le succès économique - même si tardif - du réseau de East Village et du soutien de ville de Calgary au développement d'un deuxième réseau de chaleur, en partenariat avec Enmax sur un autre programme d'aménagement dense de la municipalité. Par effet miroir, la constitution d'un réseau d'acteurs mou, sans projet territorial aligné a participé aux difficultés économiques du projet de DLSC et in fine aux difficultés de le répliquer. 


\section{Transition bas-carbone, réseaux de chaleur et transformation de l'espace urbain}

Les relations à l'espace et les interprétations et stratégies spatiales adoptées par chaque acteur, résumées dans le tableau 5, expliquent pourquoi, bien qu'évoluant dans un même environnement urbain et économique peu favorable aux réseaux de chaleur, chacun des deux projets n'a pas eu le même succès pour développer un équilibre économique pérenne et être pris comme modèle pour être répliqué.

Tableau 5. La spatialité opposée des deux projets de réseaux de chaleur

\begin{tabular}{|c|c|c|}
\hline & Réseau de Calgary & Réseau d'Okotoks \\
\hline $\begin{array}{l}\text { Intégration } \\
\text { dans l'espace } \\
\text { urbain }\end{array}$ & $\begin{array}{l}\text { Redéveloppement urbain dense } \\
\text { dans le centre-ville de Calgary } \\
\text { avec bâtiments résidentiels et } \\
\text { commerciaux. }\end{array}$ & $\begin{array}{l}\text { Développement d'un nouveau } \\
\text { lotissement monorésidentiel } \\
\text { suburbain à faible densité. }\end{array}$ \\
\hline $\begin{array}{l}\text { Stratégies } \\
\text { territoriales } \\
\text { sur le réseau }\end{array}$ & $\begin{array}{l}\text { Extension territoriale du } \\
\text { réseau : flexibilité dans le } \\
\text { rattachement de nouveaux } \\
\text { bâtiments et dans la fourniture } \\
\text { de chaleur. } \\
\text { Mesure du territoire : } \\
\text { cartographie du calendrier de } \\
\text { remplacement des chaudières } \\
\text { dans la zone pouvant être } \\
\text { desservie par le réseau de } \\
\text { chaleur. } \\
\text { Différenciation territoriale : } \\
\text { catégorisation du territoire en } \\
\text { zone dense et non dense et } \\
\text { ciblage des zones denses. }\end{array}$ & $\begin{array}{l}\text { Insularité : projets circonscrits } \\
\text { et clos sans possibilité de } \\
\text { nouvelle connexion et de } \\
\text { flexibilité dans l'augmentation } \\
\text { de la provision de chaleur, } \\
\text { d'extension. } \\
\text { Dépendance territoriale : } \\
\text { raccordement au système de } \\
\text { chauffage dominant pour } \\
\text { compléter les besoins en } \\
\text { chaufffage } \\
\text { Incompatibilité territoriale : } \\
\text { projet de démonstration } \\
\text { développé sur un territoire à } \\
\text { faible densité. }\end{array}$ \\
\hline $\begin{array}{l}\text { Réseau } \\
\text { d'acteurs }\end{array}$ & $\begin{array}{l}\text { Portage du projet par un acteur } \\
\text { local. } \\
\text { Constitution d'un réseau local } \\
\text { d'acteurs fort avec intérêts } \\
\text { matériels convergents. }\end{array}$ & $\begin{array}{l}\text { Importation du projet par un } \\
\text { acteur fédéral. } \\
\text { Constitution d'un réseau } \\
\text { d'acteurs local mou avec intérêts } \\
\text { matériels divergents. }\end{array}$ \\
\hline
\end{tabular}




\begin{tabular}{|l|l|l|}
\hline & Réseau de Calgary & Réseau d'Okotoks \\
\hline & $\begin{array}{l}\text { Alignement avec la stratégie } \\
\text { territoriale de la ville de } \\
\text { Calgary } \\
\text { Contrôle et suivi de la densité } \\
\text { du territoire urbain : réseaux } \\
\text { de chaleur comme indicateur } \\
\text { chaleur et } \\
\text { projet de } \\
\text { territoire } \\
\text { densification urbaine. } \\
\text { institutionnalisation des réseaux } \\
\text { de chaleur dans des plans de } \\
\text { développement urbain }\end{array}$ & $\begin{array}{l}\text { Déconnexion territoriale : } \\
\text { prouesse technologique dans } \\
\text { le secteur énergétique sans lien } \\
\text { avec l'aménagement urbain. }\end{array}$ \\
\hline $\begin{array}{l}\text { Substance de } \\
\text { la transition }\end{array}$ & $\begin{array}{l}\text { Transformation de l'espace } \\
\text { urbain en ville compacte }\end{array}$ & $\begin{array}{l}\text { Reproduction de l'espace urbain } \\
\text { des suburbs. }\end{array}$ \\
\hline
\end{tabular}

Source : auteur.

La justification à la mise en œuvre de ces deux projets et l'attribution de financements publics était la réduction attendue des émissions de carbone. En évaluant les bénéfices directs des deux projets sur le court terme, le projet de DLSC semble plus probant que le projet de Calgary en termes de réductions des GES. Le nombre de tonnes de carbone évité par le système de DLSC a été minutieusement quantifié dans le cadre du projet de démonstration : cinq tonnes de carbone par maison et par an. À l'inverse, il n'y a eu aucune communication sur la baisse des émissions de carbone liée au projet de Calgary. En outre, la pierre angulaire du projet, la centrale cogénération au gaz, a été suspendue. L'absence de données et la non-construction de la centrale à cogénération ne permettent pas d'évaluer les bénéfices environnementaux du système de Calgary et peuvent interroger sur la pertinence environnementale du projet.

Néanmoins, cette approche des bénéfices environnementaux directs sur l'ensemble du projet doit être remise en cause et relativisée en changeant l'échelle spatio-temporelle. Premièrement, une observation à une échelle plus fine du système de DLSC met en évidence que, sur cinq tonnes de carbone évitées, deux sont dues à l'efficacité énergétique des habitations. Les dépenses supplémentaires liées à l'isolation thermique des maisons n'ont représenté que $5 \% \mathrm{du}$ coût global du projet (\$CAD 7 millions) (voir tableau 2). Le coût et la 
faible réduction des émissions de carbone du dispositif de stockage saisonnier de chaleur solaire posent la question de la légitimité de ce choix technologique. L'analyse coûts/bénéfices des differents éléments du projet soulève la question de quelle partie matérielle répliquer : le système de production de chaleur ou les standards d'isolation thermique des maisons ? Or cette dernière dimension d'économie des consommations est très peu mentionnée dans les discours sur le projet.

Deuxièmement, une analyse de long terme et à une échelle plus large montre que le projet de Calgary, qui s'inscrit dans un projet territorial de densification de l'espace urbain, semble avoir des bénéfices environnementaux indirects plus importants que le projet d'Okotoks, qui ne remet pas en cause le modèle pavillonnaire d'étalement urbain. La ville compacte, avec bâti dense, mixité fonctionnelle et transport public, émet moins de gaz à effet de serre que le développement de pavillon individuel dans des zones monorésidentielles centrées sur l'automobile.

Plus généralement, la comparaison entre ces deux projets de réseaux de chaleur, de leur relation à l'espace urbain (comme outil stratégique manipulable et comme terrain d'ancrage), et l'analyse critique de leurs bénéfices environnementaux, soulève la question de la relation entre transitions bas-carbone et capacité de transformation de l'espace urbain. L'incapacité à impliquer financièrement ou politiquement les acteurs locaux, l'insularité du réseau, l'incohérence technique du projet et l'absence de construction d'un projet territorial compatible avec les exigences d'économie des réseaux de chaleur n’ont pas permis au réseau de DLSC de développer un modèle économique viable ni de créer des relais locaux de réplication. Les caractéristiques spatiales du système de DLSC impliquent une dissociation entre le système énergétique et le contexte urbain dans lequel il s'inscrit. Tous deux sont considérés comme indépendants l'un de l'autre. L'absence de remise en cause de l'espace urbain existant ne permet une transformation.

À l'inverse, la capacité de former une alliance forte entre trois acteurs locaux, l'extension territoriale du réseau, la prise en compte de l'hétérogénéité du territoire et l'ancrage dans un projet de densification de l'espace urbain, ont permis au système de Calgary d'atteindre un équilibre économique et de développer un projet de réplication. Par 
conséquent, le projet de Calgary intègre simultanément la coévolution de ces deux systèmes sociotechniques, ce qui crée des relais de réplication chez les acteurs de l'aménagement urbain. Le modèle du réseau de Calgary ne transforme pas uniquement le régime de chauffage. Son utilisation par la ville à la fois comme un indicateur et un instrument de densification, ainsi que le travail institutionnel qu'Enmax entreprend auprès des aménageurs et des services municipaux, participent aussi à la transformation de l'espace urbain dominant et aux pratiques d'aménagements urbains. Ces nouvelles pratiques, bien que poussées par des considérations commerciales (diversification des activités d'Enmax) et budgétaires (déficit anticipé de la Ville pour pourvoir aux besoins d'infrastructures), ont des effets environnementaux structurels bien plus conséquents que le projet d'Okotoks.

Les réseaux de chaleur sur le modèle de celui d'East Village gagnent un consensus institutionnel. En plus du projet de West Village, la ville d'Edmonton, capitale de la province, envisage également un réseau de chaleur dans un programme de réaménagement dense situé près de l'aéroport. Ces systèmes alternatifs de provision de chaleur ne sont plus perçus comme une innovation de niche, mais sont en train de se constituer lentement comme un outil d'aménagement urbain durable modifiant le paysage urbain, mais également le paysage des pratiques urbaines.

Néanmoins, le frein principal demeure le faible prix du gaz. Sans une augmentation durable des prix des énergies fossiles (soit par les marchés, soit par l'introduction d'un prix incitatif du carbone), beaucoup de projets auront des difficultés à être économiques.

S’intéresser aux transitions des systèmes sociotechniques nécessite de conceptualiser l'espace, d'une part comme un instrument stratégique interprété et utilisé par les acteurs afin d'avancer leurs intérêts propres, et d'autre part comme un terrain structurel qui peut être transformé sur le long terme par les acteurs en fonctions des stratégies mobilisées. Les concepts de territoire, de réseaux et d'échelle aident à structurer ces stratégies et à les comparer. La spatialité propre à chaque réseau explique que dans un même contexte économique défavorable, le projet de Calgary a réussi à développer un modèle économique viable et à être bientôt répliqué, alors que celui d'Okotoks a concentré l'attention sur ses coûts exorbitants et n'a toujours pas fait d'émule. 
Cette comparaison montre également que la transition des systèmes sociotechniques n'est pas un simple enjeu de substitution technologique a-spatialisée. Prendre pour objet la diffusion ou la transformation des infrastructures urbaines dans le cadre d'une transition soutenable, c'est remettre en question l'ensemble de l'espace urbain et des pratiques urbaines, et remettre au cœur de l'analyse l'appropriation de l'espace comme terrain d'actions et comme terrain à transformer. Cette interprétation de la transition renoue avec la géographie critique lefebvrienne (Lefebvre, 1974) et permet de conceptualiser la diffusion d'une innovation de niche comme étant le produit de politiques, de pratiques et de systèmes qui vont au-delà des considérations environnementales. L'espace permet également d'inscrire la problématique de diffusion et de transition soutenable en lien avec d'autres enjeux territoriaux.

\section{Références}

Chappin E. J. L., Ligtvoet A. (2014), « Transition and transformation: A bibliometric analysis of two scientific networks researching sociotechnical change ", Renewable and Sustainable Energy Reviews, ${ }^{\circ}$ 30, p. 715-723.

CIEEDAC (2014), District Energy Inventory For Canada, 2013, Burnaby (Colombie-Britannique), Simon Fraser University.

City of Calgary (2009), Calgary's Municipal Development Plan.

City of Calgary (2011a), Calgary Community GHG Reduction Plan - Energy in the City.

City of Calgary (2011b). Long Range Financial Plan. The City of Calgary.

City of Calgary (2013), Municipal Development Plan / Calgary Transportation Plan: 2013 Monitoring Progress Report.

Coenen L., Truffer B. (2012), "Places and Spaces of Sustainability Transitions: Geographical Contributions to an Emerging Research and Policy Field ", European Planning Studies, vol. 20, n 3, p. 367-374.

DLSC (2015), Site officiel de Drake Landing Solar Community, http:// www.dlsc.ca/how.htm (consulté le 11 juillet 2017). 
Dodson J. (2013), "Suburbia under an Energy Transition: A Sociotechnical Perspective ", Urban Studies, vol. 51, n 7, p. 1487-1505.

Geels F. W., Schot J. (2007), "Typology of sociotechnical transition pathways ", Research Policy, vol. 36, n 3, p. 399-417.

Geels F. W., Schot J. (2010), "The dynamics of transitions: a socio-technical perspective ", dans Grin J., Rotmans J., Schot J. (éds), Transitions to Sustainable Development, New York-London, Routledge, p. 11-101.

Grin J., Rotmans J., Schot J. (2010), « From persistent problems to system innovations and transitions ", dans Grin J., Rotmans J., Schot J. (éds), Transitions to Sustainable Development, New York-London, Routledge, p. 1-9.

INSEE (2011), http://www.insee.fr/fr/themes/comparateur.asp?codgeo=de p-75 (consulté le 11 juillet 2017).

Krueger R., Agyeman J. (2005), "Sustainability schizophrenia or "actually existing sustainabilities?" toward a broader understanding of the politics and promise of local sustainability in the US ", Geoforum, vol. 36, n ${ }^{\circ} 4$, p. 410-417.

Lefebvre H. (1974), La production de l'espace ( $4^{\mathrm{e}}$ éd.), Paris, Anthropos, 2000.

Markard J., Raven R., Truffer B. (2012), "Sustainability transitions: An emerging field of research and its prospects ", Research Policy, vol. 41, $\mathrm{n}^{\circ}$ 6, p. 955-967.

NRCAN (2012), "Ressources naturelles Canada, Secteur résidentiel. Base de données complète sur la consommation d'énergie. Tableau 9 : Émissions de GES pour le chauffage des locaux par type de système ", http://oee.nrcan.gc.ca/organisme/statistiques/bnce/apd/showTable.cfm ?type $=\mathrm{CP} \&$ sector $=$ res $\&$ juris $=\mathrm{ab} \& \mathrm{rn}=9$ \&page $=3 \&$ attr $=0 \quad$ (consulté le 11 juillet 2017).

Okotoks (2015), "Distinction pour la gestion de l'eau ", http://www. okotoks.ca/municipal-government/newsroom/news/2015-fcmsustainable-communities-award-winners (consulté le 11 juillet 2017).

Okotoks (2015), "Plan de gestion de l'eau », http://www.okotoks.ca/ sustainability/environment/water-efficiency-conservation (consulté le 11 juillet 2017). 
Raven R., Schot J., Berkhout F. (2012), « Space and scale in socio-technical transitions ", Environmental Innovation and Societal Transitions, vol. 4, p. 63-78.

Read A. (2014), Climate change policy in Alberta, Pembina Institute.

Statistique Canada (2011a), "Population urbaine et rurale, par province et territoire (Alberta) ", http://www.statcan.gc.ca/tables-tableaux/sumsom/102/cst01/demo62j-fra.htm (consulté le 11 juillet 2017).

Statistique Canada (2011b), "Série "Perspective géographique". Recensement de 2011 ", www12.statcan.gc.ca/census-recensement/2011/as-sa/ fogs-spg/Facts-pr-fra.cfm?Lang=fra \&GK=PR\&GC=48\#FN-DQF (consulté le 11 juillet 2017).

Statistique Canada (2011c), "Recensement de 2011, Ville de Calgary ", http://www12.statcan.gc.ca/census-recensement/2011/dp-pd/prof/ details $/$ page. .fm? Lang $=\mathrm{E} \& \mathrm{Geo} 1=\mathrm{CSD} \&$ Code $1=4806016 \& \mathrm{Geo} 2=\mathrm{P}$ R \&Code $2=48 \&$ Data $=$ Count $\&$ Search Text $=$ Calgary $\&$ Search Type $=$ B egins \&SearchPR $=01 \&$ B 1 $=$ All \&GeoLevel $=$ PR\&GeoCode $=4806016$ (consulté le 11 juillet 2017).

Statistique Canada (2011d), "Recensement de 2011, Ville d'Okotoks ", http://www12.statcan.gc.ca/census-recensement/2011/dp-pd/prof/ details/page. .fm? Lang $=\mathrm{E} \& \mathrm{Geo} 1=\mathrm{CSD} \& \mathrm{Code} 1=4806012 \& \mathrm{Geo} 2=\mathrm{C}$ D\&Code2 $=4806 \&$ Data $=$ Count $\&$ Search Text $=$ okotoks $\&$ Search Type $=$ Begins $\&$ SearchPR $=01 \& \mathrm{~B} 1=$ All $\&$ Custom $=\& \mathrm{TABID}=1 \quad$ (consulté le 11 juillet 2017).

Town of Okotoks (2007), « Sustainable Okotoks "Leave a Legacy”, présentation pour la Fédération canadienne des Municipalités », 29, 3 août 2007.

Town of Okotoks (2016), Okotoks Community Sustainability Plan 20162019.

Truffer B., Coenen, L. (2012), « Environmental Innovation and Sustainability Transitions in Regional Studies ", Regional Studies, vol. 46, $\mathrm{n}^{\circ} 1$, p. 1-21.

Vivien F.-D. (2005), Le développement soutenable, Paris, La Découverte, coll. "Repères ". 


\section{LES INFRASTRUCTURES DE GESTION}





\title{
Les assemblages d'outils de gestion comme infrastructures de connaissance
}

\author{
Gilles Jeannot \\ Pascal Ughetto
}

Parmi les très nombreuses analyses sociologiques menées sur les outils de gestion dans les entreprises et les administrations (Chiappello et Gilbert, 2013), la plupart ont isolé un outil au sein d'un ensemble foisonnant. Ce parti pris n'est pas nécessairement réaliste tant ces outils de gestion ne sont jamais isolés dans les entreprises. Ce sont de très nombreux cadres formalisés qui sont simultanément utilisés dans les entreprises et les administrations, accumulés, mis en concurrence, articulés, intégrés. Cette question a été récemment abordée à propos du cas particulier des progiciels intégrés de gestion ou enterprise ressource planning (ERP) ; elle mérite d'être appliquée de manière plus large à différentes modalités de compositions d'outils de gestion. La manière dont différents outils de gestion sont mis en relation et ce qui en résulte - ce que nous appellerons en première approche et de manière ouverte " assemblages"-, est ainsi un sujet important pour comprendre la gestion technique des entreprises et des administrations. Comment ces " assemblages " émergent-ils ? Qu'est-ce qui les fait tenir ? Quels effets autres que ceux de leurs composantes ont-ils?

Pour répondre à l'invitation de produire un article sur les infrastructures à partir d'un domaine, celui de la gestion des organisations privées ou publiques, dans lequel cette notion est peu mobilisée, ces assemblages d'outils de gestion semblent pouvoir en première intuition constituer des candidats honorables pour jouer de la métaphore. En effet, si on s'arrête au cas des progiciels intégrés de gestion, ces macrologiciels multifonction apparaissent par leur taille et leur apparente compacité comme un support essentiel au fonctionnement de l'entreprise, une infrastructure, de la même manière qu'un réseau électrique ou routier supporte les services associés. D’ailleurs, Nonjon et Marrel (2015) ont 
recours à une image proche, empruntée au vocabulaire professionnel des informaticiens, celle d'" architecture informatique de gestion ». Nous avons donc emprunté sur les étagères - toutes virtuelles - de nos collègues quelques ouvrages classiques sur les infrastructures (Hughes, Bower et Star, Edwards) et nous nous sommes attachés à repérer des similitudes ou des différences. La mise en regard des théories de l'infrastructure et des quelques connaissances glanées sur ces assemblages d'outils de gestion, nous a conduits alors sur une autre voie que celle de l'intuition initiale. Ce détour de lecture nous a permis en effet de questionner tout ce qui se cache de dynamique et d'inachevé derrière cette apparente solidité et compacité. Pour les auteurs qui se sont penchés sur la notion d'infrastructure, quand l'infrastructure est un succès, ces éléments se consolident mutuellement autour d'un axe principal, donnant l'impression d'un tout capable d'une forte résistance aux épreuves. Mais, en réalité, l'infrastructure doit beaucoup à des modules, des sousensembles, des constituants d'origines diverses. Comme le note Paul N. Edwards à propos des " knowledge infrastructures" :

"Key to the infrastructure perspective is their modular, multi-layered, rough-cut character. Infrastructures are not systems, in the sense of fully coherent, deliberately engineered, end-to-end processes. Rather, infrastructures are ecologies or complex adaptive systems; they consist of numerous systems, each with unique origins and goals, which are made to interoperate by means of standards, socket layers, social practices, norms, and individual behaviors that smooth out the connections among them " (Edwards et al., 2012, p. 5).

Partons donc explorer les outils de gestion comme assemblages et infrastructures de connaissance.

\section{Des assemblages d'outils de gestion?}

Si la recherche sociologique sur les outils de gestion a été très largement développée ces dernières années et a pris une part importante dans la compréhension des organisations productives, la question de l'association des outils entre eux, des formes de configurations, des causes d'émergence de ces configurations et de leurs effets a encore peu attiré l'attention. 
Dans l'ouvrage pédagogique qu'ils consacrent à la sociologie des outils de gestion, Ève Chiapello et Patrick Gilbert présentent de manière très synthétique un vaste ensemble d'articles qui illustrent les différentes thèses sociologiques sur les outils de gestion. Ce faisant, ils ont fait l'effort de caractériser non seulement l'argument soutenu, mais aussi l'objet particulier auquel les auteurs de ces articles les appliquent. Quelques références portent sur un cadre amont global prédéterminant des outils de gestion (comme les nomenclatures statistiques analysées par Desrosières) et quelques autres s'aventurent du côté de la recherche d'un effet global des outils de gestion, par exemple dans la sociologie clinique. Même dans la partie consacrée aux tenants de l'acteur réseau, le réseau étudié semble être celui qui se tisse autour d'un outil particulier et non entre les différents outils (le seul exemple se rapprochant dans ce groupe d'une infrastructure de connaissance pourrait être l'analyse par Franck Cochoy des modalités d'exposition des biens dans les supermarchés étagères, packaging, étiquettes...--, mais on est là aux limites des outils de gestion).

La principale exception à cette approche centrée sur un outil concerne les progiciels intégrés de gestion (PGI ou ERP en anglais) qui sous l'apparence d'un outil unique sont en fait un collage de fonctions multiples qui dans les formes idéales pourraient même recouvrir la plupart des fonctions de l'entreprise. Outre les références citées dans cet ouvrage de synthèse, on peut citer trois numéros spéciaux des revues Sciences de la société (Segrestin, 2004), Réseaux (Kocoglu et Moatti, 2010) et Gouvernement et action publique (Nonjon et Marrel, 2015). Les ERP peuvent être considérés comme des assemblages de gestion. Ces logiciels prétendent réunir en un seul outil des traitements de données initialement totalement distincts. Se présentant sous la forme de modules, ils doivent, par ailleurs, établir des points de passage avec des applications informatiques qui ne passent pas sous le joug de l'ERP. En fait, les ERP relèvent d'une conception et d'une implantation en entreprise structurées par l'ambition d'harmoniser des données qui étaient de formats différents. Ils s'accompagnent d'un travail généralement lourd - de recodification des données que, à partir de logiques professionnelles distinctes, des secteurs (achats, comptabilité, approvisionnement...) envisageaient dans des termes spécifiques. Il s’agit de faire passer ces données sous l'hégémonie de systèmes de catégories 
unifiées qui se veulent moins locaux, plus transversaux. Très souvent, l'implantation d'un ERP nécessite au préalable une réorganisation, pour configurer l'organisation en réponse aux attentes de l'outil technique. C'est ce qui a donné lieu, dans les entreprises et les administrations concernées, à une réception dominée par la critique d' "usines à gaz ", de systèmes lourds et peut-être inutilement contraignants. Les ERP se sont attiré la critique des métiers dont ils remettaient en cause, non seulement les outils qui étaient familiers à leurs représentants, mais les modes d'organisation et les représentations.

La notion d'assemblage d'outils de gestion doit aussi être étendue à des ensembles d'outils qui ne sont pas mis en relation par un artefact informatique, mais par une doctrine qui les présente comme complémentaires. Le lean management illustre bien cette forme d'assemblages d'outils de gestion. Pratiquer le lean management dans l'industrie, c'est en effet appliquer simultanément du kaïzen, du poka yoke, des cartes kan ban, du 5S, du value stream mapping, du management visuel, du traçage des actes permettant d'identifier des mudas (de temps d'attente, de double travail, etc.). Les consultants demandent d'y voir des composants qui se " clipsent " ensemble et qu'il faut nécessairement réunir pour remplacer les formes existantes d'encadrement de la production.

Enfin, il est peut-être pertinent d'étendre encore cette idée d'assemblage, au-delà de l'architecture informatique ou de l'association doctrinale, à la régularité statistique des associations d'outils. Les économistes qui mobilisent des enquêtes par questionnaire sur la diffusion des pratiques de gestion rendent disponible un matériau qui échappe au tropisme de la monographie par outil dominant dans la sociologie des outils de gestion. Cela ne signifie pas que la question des assemblages d'outils soit pour autant pensée comme telle. En effet, lorsque les économistes dénombrent des outils de gestion, ce n'est pas à proprement parler pour étudier leur diffusion, leurs usages et impacts, mais comme indicateurs d'une autre dimension plus large, celle du changement organisationnel (Greenan et al., 2016). À défaut d'une discussion explicite des regroupements d'outils de gestion, on trouve dans la méthode retenue une manière d'appréhender de fait ce regroupement. Deux arguments sont mobilisés de fait : une appréciation synthétique globale des outils qui correspond à la représentation d'une intensité 
globale d'engagement dans les nouvelles pratiques gestionnaires (et/ ou informatiques) et un ensemble de contraintes qui sont susceptibles d'infléchir l'adoption de ces outils dans une perspective théorique de la contingence (effets de taille, de secteur...).

Les données empiriques produites par les économistes peuvent être mobilisées indirectement cependant pour répondre à la question qui nous occupe : les relations établies entre les différents outils mobilisés par une entreprise. Un résultat empirique central s'impose : lorsque l'on fait une analyse factorielle, un premier axe correspondant à l'opposition entre entreprises utilisant les outils et entreprises ne les utilisant pas exprime une forte inertie. Cela signifie que le premier facteur différenciant les entreprises est leur engagement global dans l'usage des outils. On peut aussi considérer que les outils sont classés dans une liste d'outils de plus en plus sophistiqués que les entreprises s'approprient progressivement (Jeannot et Guillemot, 2010). Ces données statiques sont complétées par des données dynamiques. D'une part, hormis pour les systèmes intégrés, les entreprises n'abandonnent pas un outil qu'elles ont adopté. D'autre part, les études longitudinales montrent des diffusions globales progressives des outils (Greenan et Walkoviak, 2010). Ces données globales par enquêtes sont intéressantes, car elles permettent de mettre en évidence les associations entre les outils de gestion, mais elles ne permettent de qualifier que très partiellement les interactions entre ces outils qui coexistent au sein des entreprises et l'écologie de leur développement. Elles suggèrent que les entreprises n'introduisent pas les outils de manière désordonnée, sans faire jouer des formes d'association et de cohérence, qui restent à comprendre.

Les trajectoires de modernisation telles qu'on peut les observer ne se font ainsi pas de manière désordonnée : des outils sont souvent pris dans des assemblages, qu'ils prennent la forme d'architecture informatisée, d'associations projetées dans une théorie ou de complémentarités moins anticipées. Cela n'empêche pas toutefois de réelles difficultés de cohabitation : dans les formes les moins organisées, avec des concurrences entre prescripteurs, et dans les formes les plus organisées, avec une résistance au contrôle de cohérence ou d'interopérabilité. Étudier la dynamique de développement et d'usage de ces assemblages de gestion est ainsi le plus sûr moyen d'éviter d'imaginer ces dernières comme des substances parfaitement cohérentes, apportant des 
réponses évidentes à des besoins non moins évidents et faisant usage de composants facilement compatibles entre eux, sans difficulté technique et sans possibilité pour la controverse politique de s'immiscer dans les choix techniques.

C'est alors que la relecture de travaux sur les infrastructures peut aiguiser le regard. Nous discuterons ici plusieurs auteurs qui ont abordé la notion d'infrastructure pour voir comment les questions qu'ils posent peuvent éclairer l'analyse des assemblages de gestion. Nous aborderons d'abord les travaux de Hughes qui ont porté sur des infrastructures traditionnelles et en l'occurrence le réseau électrique. Puis nous lirons ceux de Bowker et Star qui se sont interrogés sur les questions de standards dans la constitution d'assemblages entre composantes d'une infrastructure. Nous nous pencherons enfin sur les travaux d'Edwards et de ses collègues qui, en partant du cas de l'Internet, ont opéré un déplacement métaphorique en discutant $d$ ' "infrastructures de la connaissance ». Paul N. Edwards a défini les « knowledge infrastructures » comme des " robust networks of people, artifacts and institutions that generate, share, and maintain specific knowledge about the human and natural world " (Edwards et al., 2012). L'ensemble de ce qui permet la météo, des capteurs et ordinateurs de traitement aux exploitations sophistiquées pour l'aéronautique ou aux multiples sites dédiés qui la diffusent dans le grand public, peut être considéré par exemple comme une « knowledge infrastructure ». Le but n'est pas de faire entrer à tout prix les questions posées par ces outils de gestion et leurs associations dans les catégories établies par ces auteurs, mais de prendre le temps de mesurer ce qui colle et ne colle pas.

\section{Assemblage d'outils de gestion et développement des infrastructures}

Dans Networks of Power, T. P. Hughes a développé à propos du cas de l'électricité une réflexion sur la naissance et le développement des infrastructures qui peut valoir bien au-delà de cet exemple. 


\section{Momentum}

Un premier argument développé par T. P. Hughes (1993) à propos des infrastructures est que les investissements, à un moment donné, génèrent de l'inertie par rapport à un modèle et tendent à engager durablement les successeurs sur les mêmes voies. Hughes parle ainsi du momentum, cette masse et cette direction que le projectile lancé conserve et qu'il serait lourd de vouloir combattre. Cet argument illustré sur des réseaux matériels, pour lesquels la fixation de capital est très importante, est, note Edwards, potentiellement moins important pour les infrastructures de la connaissance. Cependant, l'argument mérite d'être discuté, car, précisément, il pose la question de savoir si, parce qu'il s'agirait moins d'investissements matériels, les outils de gestion s'affranchiraient de ces pesanteurs et autoriseraient à sauter de mode en mode très facilement. L'idée de la mode valorisant le nouveau quand celle de l'inertie valorise l'ancien, les deux principes semblent contradictoires.

Cette opposition ne vaut cependant que pour le sens commun. Chez un auteur de référence comme Abrahamson (1991), la notion de mode managériale est plus riche que cela. La mode managériale est, en quelque sorte, une proposition d'interprétation des problèmes productifs et, plus largement, économiques, auxquels les entreprises se confrontent ; elle constitue une part d'un sensemaking (Weick, 1995) permettant de lever l'énigme, la difficulté à comprendre ce qui leur arrive et ce qu'il conviendrait de faire ; et elle est, simultanément, une proposition de façon d'intervenir sur le réel pour dominer ou réduire la difficulté, rendre l'environnement plus conciliant avec les projets et les besoins de l'entreprise et de sa stratégie. On peut inférer de ce que développe Abrahamson l'idée qu'il n'y a pas de mode managériale qui ne suppose avant, pendant et après, un travail intense des acteurs d'entreprises, et plus spécifiquement des acteurs des directions, pour faire sens de ce que l'entreprise rencontre comme difficultés (dans ses ventes, son taux de pénétration du marché, son succès auprès de telle ou telle clientèle, dans les comportements de ses salariés, etc.) et pour concevoir la façon dont la mode pourrait représenter une solution en interne, à quels prix d'adaptation, de correction de l'organisation ou des techniques existantes, d'ajustement de la méthode que recouvre la mode managériale, etc. 
Cette contradiction peut aussi être levée, de manière plus pratique, si on note qu'un outil peut être plus ou moins investi au cours du temps et que, par ailleurs, les enquêtes montrent qu'à la seule exception des ERP, les entreprises n'abandonnent pas des outils qu'elles posséderaient. La mode porterait ainsi sur les nouveaux outils, mais les anciens outils continuent à exister dans l'entreprise. De fait, même si les innovations de gestion sont portées par des logiques de développement qui sont la mode managériale, elles recouvrent bel et bien des investissements, souvent très lourds, au minimum en termes d'heures de conception et de déploiement, voire de matériels. Elles peuvent également s'être cristallisées dans des schémas d'organisation, coûteux à remettre en cause. Enfin, elles font système entre elles et il est parfois difficile de revenir dessus sans devoir bouleverser, de façon coûteuse, d'autres composantes : autres outils, organisation, etc.

\section{Hérisson}

Un deuxième argument de Hughes est que, en particulier pour dépasser les obstacles à leur généralisation (reverse salients), il faut des individus qui peuvent prendre en compte toutes les dimensions économiques et sociales de ces infrastructures et proposer des solutions ajustées qui combinent de façon pertinente ces différentes dimensions ; qui insistent comme des hérissons plutôt que de changer de sujet comme les renards.

Dans le domaine de l'entreprise, est-ce ainsi que l'on pourrait voir, par exemple, un ingénieur comme Ohno ? N'est-il pas une sorte de hérisson à l'intérieur de Toyota. C'est à regarder de près. D'un côté, celui qui va inventer le système d'organisation Toyota (aujourd'hui popularisé sous le nom d'organisation lean) est un ingénieur confronté au problème de devoir lancer une production d'automobiles avec immédiatement l'obstacle de l'absence de perspectives de vente susceptibles de générer de réelles économies d'échelle. Toyota tente bien, lors de la construction de sa première usine en 1938, d'implanter les techniques d'une organisation fordienne, mais "Toyota n'a pu installer le système fordien que partiellement sous les contraintes de financement et de marché " (Shimizu, 1999, p. 6) : les machines-outils sont bien spécialisées, mais en les rendant capables de s'ajuster à une variété de modèles de manière à pouvoir les utiliser longtemps ; plutôt 
que de pousser loin la standardisation des tâches et leur parcellisation, on crée des procédés simplifiés de fabrication ; ou encore, par manque de ressources financières, on se contente d'installer des convoyeurs dans une partie seulement des ateliers et non dans tous (ibid.).

De sa fonction de chef de l'atelier de mécanique en 1949 à la direction de l'entreprise, Taiichi Ohno va travailler de manière persévérante sur cet unique problème qu'est de constituer des conditions économiques aussi rentables que l'organisation fordienne dès lors qu'on ne se donne pas les effets d'échelle comme base possible ou principale de productivité, mais certainement pas en se posant et en inventant, en une seule opération, le schéma intellectuel et pratique d'une méthode alternative au fordisme. Il invente une première solution à un problème circonscrit, en mesure les effets positifs, mais aussi les inconvénients nouveaux que cela fait naitre ; il mûrit la conception d'une réponse à ce problème par un dispositif supplémentaire, et ainsi de suite. Ainsi, il pose le principe d'une autonomisation des équipements :

«En se demandant [...] pourquoi, chez Toyota, une personne ne conduit qu'une seule machine, Taiichi Ohno essaie vers 1947 de faire opérer un ouvrier sur deux ou trois machines-outils à la fois afin d'augmenter la productivité. Pour ce faire, il semble que lui soient venues l'idée de l'autonomisation des machines-outils et l'invention des dispositifs nécessaires. Car, pendant qu'un ouvrier travaille sur une des deux machines-outils, l'autre doit automatiquement pouvoir fonctionner et s’arrêter dès qu'il a fini le façonnage. Pour cela, la machine-outil est équipée d'un dispositif, limit switch, qui l'arrête quand le nombre de pièces ouvrées atteint un seuil déterminé. [...] Partant de là, se développe le concept de la ligne de fabrication et des mesures telles que poka yoke, c'est-à-dire le dispositif qui, par exemple, immobilise la machine-outil quand une pièce à œuvrer n’est pas correctement posée [...] " (ibid., p. 14).

Des principes (autonomisation des lignes de fabrication, nivellement de la production, production mixte) sont inventés et des dispositifs sont élaborés pour les traduire en pratique. Ohno invente, par conséquent, des solutions qui n'ont pas prétention à être des pièces d'un système parfaitement maîtrisé ni universel, mais des dispositifs qui ne révèlent une cohérence qu'après coup et d'une pertinence interne à l'entreprise pour laquelle il travaille. Quand les consultants se font, aujourd'hui, promoteurs de la mode managériale lean, ils prétendent, au contraire, 
avoir trouvé une excellente infrastructure, qui est à transposer telle quelle, tout comme le fordisme s'imposait, à l'époque où Toyota ouvrait ses premières usines, comme une grappe de dispositifs à mettre en œuvre de façon cohérente.

\section{Convergence des formats}

Bowker et Star (1999) ont mis au jour l'enjeu que représente le travail permettant de dépasser une situation où des formats extrêmement nombreux et divers, sans coordination particulière, posent des limites à l'interopérabilité, aux transferts de données, à la circulation de ces données, etc. Edwards, Bowker, Jackson et Williams (2009), eux aussi, se montrent circonspects à l'égard d'une " convergence numérique " (digital convergence) d'ores et déjà parfaitement réalisée, et cela par l'argument suivant : "A colossal cacophony of differing data formats, access methods, security systems, and intellectual property restrictions still places us very far from the universal libraries and information utilities of visionaries » (p. 366). Les infrastructures passent donc d'abord par un intense travail de codification, de catégorisations et d'extension de l'échelle d'application de ces catégories, de création de standards, y compris de standards et d'outils de type gateway (ibid., p. 367) permettant à des systèmes d'interopérer. Comment cela marchet-il pour des outils de gestion dont il a pu être question ces dernières années ? Peut-être est-il intéressant ici de mobiliser de nouveau ces deux exemples, de nature très contrastée, que sont les ERP et le lean.

Les ERP ont justement été constitués autour du projet de l'unification des formats des données, qui permettrait une saisie unique des informations. Kocoglu et Moatty (2012) ont montré qu'il n'existe pas un processus, chaque fois identique, d'implantation d'ERP, mais des configurations dans lesquelles, à travers les modules retenus, on intègre un très petit nombre de fonctions au sein de l'entreprise (par exemple, deux), d'autres où l'on vise l'intégration totale (cinq ou six fonctions). La plupart du temps, l'intégration est partielle : intégration de production, comptabilité, achats, ventes, ou de production et comptabilité, de comptabilité, achats et ventes ou de production, achats et ventes. Même dans ce cadre, les difficultés ne sont pas minces pour parvenir à associer les modules, paramétrer, tenir la stabilité des données, etc. 
Dans le lean, également, on l'a vu, une modularité et de la sélectivité existent. Les assemblages peuvent être variés. Dans certains cas, une entreprise implantera le lean en considérant qu'un de ses dispositifs pivot est le calcul du Takt time; dans d'autres cas, on pourra observer des applications du lean où le Takt time n'est pas une obsession, voire n'est pas même évoquée (Ughetto, 2009). Dans l'essor, au cours des dernières années, du lean dans les univers tertiaires (entreprises de services ou bureaux des entreprises industrielles), il est éventuellement question de value stream mapping (cartographie des circulations des documents et des personnes pour réduire les déplacements inutiles) ou de management visuel (indication par des jeux de couleurs de lieux de dépôt de documents, par exemple), mais généralement pas de kan ban, qui pourra pourtant être considéré dans la doctrine initiale comme une composante clé. Il est d'ailleurs notable que, d'un auteur à l'autre, parmi ceux qui ont contribué à élaborer la doctrine et à la promouvoir, aucun n'établit point par point la même liste de composants de la méthode. Et cela est redoublé quand on observe le lean en train de s'importer dans une entreprise, processus où il est bien question de savoir ce que l'on doit remettre en cause de l'existant, ce qui va être conservé et ce qui va permettre de créer des sortes de passerelles entre ce qui demeure et les dispositifs lean. Ceux-ci vont en réalité s'articuler à des assemblages d'outils préalables qui ne seront certainement pas remis en cause.

Une différence est peut-être que les ERP exigent des opérations de réorganisation d'autant moins négociables qu'elles sont imposées par l'informatique, qui agit puissamment sur les catégorisations. Le lean exige également que les entreprises fassent le ménage dans les catégories incompatibles avec la doctrine et les outils, mais ces outils ne sont pas aussi intolérants avec l'existant que peut l'être un ERP. Pour s'exprimer à la manière de W.J. Orlikowski (1992), certaines infrastructures ont ainsi - comme les ERP - une faible flexibilité interprétative ; d'autres - comme le lean - manifestent au contraire une plus forte flexibilité de ce type. 


\section{Les assemblages d'outils de gestion comme infrastructures de connaissance}

Paul Edwards a cherché à montrer la pertinence d'une application de la notion d'infrastructure et des concepts proposés par Hughes à des entités complexes plus immatérielles, le point de départ de sa réflexion étant la naissance de l'Internet. Les outils de gestion ne sont pas loin, en effet, il cite le premier document qui propose la création du Web et qui était intitulé "Information management: a proposal ", autrement dit le Web était conçu à l'origine comme un outil de management. On connait la suite et comment cet outil a été très largement approprié et s'est développé comme infrastructure de connaissance. Ce déplacement le conduit à porter l'attention sur de nouvelles questions, la première est celle des échelles comme méthode.

\section{La superposition et l'interaction des échelles}

Dans son article (Edwards et al., 2013), il utilise, avec ses collègues, la notion d'échelle de deux manières, comme une méthode pour révéler différents aspect du développement d'une infrastructure et comme une caractéristique particulière des infrastructures qui mettraient en forme notre modernité en reliant différentes échelles. En termes de méthode, il complète le niveau méso qui est celui abordé par les approches classiques des large technical systems par un niveau micro des individus ou petits groupes à échelle de durée courte et par un niveau macro qui est celui du développement dans la longue durée, à l'échelle de sociétés, des infrastructures. De manière plus concrète, et moins banale, il pointe pour le niveau micro l'enjeu de l'appropriation locale et du développement autonome par les utilisateurs des infrastructures. Et pour le niveau macro, il remarque que, si les infrastructures peuvent mourir (exemple : le télégraphe), les fonctions associées perdurent à travers d'autres infrastructures. L'analyse macro qu'il propose consiste alors à suivre la manière dont une même fonction est prise en charge au cours du temps.

La perspective macro apparait enrichissante pour la compréhension de la diffusion du lean. Le retour du lean comme mode managériale dans le courant des années 2000 est venu des grands groupes internationaux 
désireux de découvrir une méthode garantissant d'importants gains de productivité et susceptible d'avoir valeur de stratégie défendue par la tête de groupe sur le périmètre entier du groupe. La méthode est alors imposée sans négociation possible aux filiales dans les différentes régions du monde et dans chacun des établissements au sein de celles-ci. Les modèles d'obtention de la performance sont déclinés de façon totalement top-down à leurs unités par ce qui est aujourd'hui appelé le niveau " corporate ", lequel met en concurrence les sites pour l'obtention des productions. Pour leur pilotage de leurs unités de par le monde, les grands groupes internationaux tendent donc à constituer des configurations les plus standards possibles d'assemblages d'outils de gestion, qu'il s'agisse de la grappe d'outils lean que des autres outils auxquels le lean s'articule. En réalité, l'observation montre qu’on passe par des processus d'appropriation, bien documentés également pour les ERP, se traduisant par le fait qu'il y a des interprétations nationales du lean au sein d'un même groupe (Ughetto, 2009), et, au sein des pays, des inventions établissement par établissement : cela, parce que chaque établissement n'a pas la même organisation initiale, n’a pas les mêmes productions à prendre en charge, ne présente pas les mêmes contraintes de personnel, etc. Peut-être alors la notion d'infrastructure est-elle intéressante pour arrêter le sociologue dans sa tendance à insister sur les recréations locales et tenter de tenir la tension entre une infrastructure macro, des modalités méso et des appropriations micro.

Un autre point est celui des frontières de l'entreprise. La question plus générale du lien entre infrastructure et mise en relation d'échelles différentes vaut tout particulièrement pour penser cette chose particulière qu'est l'entreprise. Jusqu'ici nous avons considéré comme allant de soi l'existence d'une organisation productive disposant d'une liste d'outils de gestion. La délimitation d'une organisation productive est cependant loin d'aller de soi : l'unité pertinente est-elle l'atelier, le site, l'entreprise? Les travaux de Gilles Crague (2014) ont attiré notre attention sur la difficulté de cerner de telles frontières. La même difficulté se retrouve concernant la définition des assemblages de gestion : faut-il les compter au niveau de l'atelier, du site ou de l'entreprise ? Et surtout est-il possible de découper selon ces niveaux ? En effet, sur un site industriel seront utilisés une base de comptabilité issue du groupe, un ensemble d'outils de suivi de la production ad hoc, un progiciel du marché et une sous- 
application maison. Ces outils ne sont pas seulement lisibles à toutes ces échelles, mais à leur manière ils permettent de relier de manière spécifique ces différents niveaux.

\section{La recomposition des pouvoirs}

Dans un autre document, Paul Edwards et un groupe de travail s'interrogent sur les recompositions de pouvoir associées à diverses possibilités de déploiement des infrastructures de savoir. La question du pouvoir est à l'évidence au cœur du déploiement des outils de gestion dans les entreprises. À une échelle limitée des différentes composantes d'un référentiel, Valérie Boussard a montré comment dans les rapports de pouvoir au sein d'une organisation, l'un des indicateurs s'impose parmi d'autres qui sont moins mobilisés dans la discussion : ce qu'elle nomme l'indicateur prégnant (Boussard, 2001). Kostas Chatzis a, quant à lui, montré comment le développement des pratiques de gestion dans les entreprises était soumis à la guerre ou à la concurrence des fonctions spécialisées (production, maintenance, logistique, qualité...) (Chatzis, 1999).

Le cas des ERP est ici tout à fait intéressant dans la mesure où ces logiciels intégrés associent toujours un argument d'économie (l'intégration des bases permet de ne pas ressaisir plusieurs fois les mêmes données) et une visée de centralisation du pouvoir, l'échelon central disposant d'une vision panoptique de l'ensemble des activités de l'entreprise. Kocoglu et Moatti (2010) notent ainsi que la fonction économique et financière est présente dans $72 \%$ des cas d'ERP et que le développement des ERP a été particulièrement fort dans le domaine de l'industrie avec la conjonction entre les fonctions d'approvisionnement et de production et les fonctions financières. Ils montrent aussi comment l'expansion de ce type d'outil rencontre des obstacles et doit par exemple prendre en compte l'existence antérieure d'un logiciel maison qui fonctionne bien et que les salariés tiennent à conserver.

\section{L'ouverture des savoirs}

Paul Edwards propose, enfin, une hypothèse transversale sur le devenir des infrastructures de connaissance. Le document collectif est construit autour de l'hypothèse d'une mutation en cours des infrastructures de 
connaissance liées à l'ouverture dans tous les sens du terme des données. L'application de cette hypothèse aux assemblages d'outils de gestion est d'autant plus intéressante que sa pertinence ne va pas de soi. En effet, les données que les entreprises produisent sur elles-mêmes sont considérées comme stratégiques et, à ce titre, secrètes. Et il n'est pas sûr que cela puisse être considéré comme un retard dans un mouvement inéluctable (les dispositions de la loi Macron du 6 août 2015 vont plutôt dans le sens d'une moindre transparence sur la comptabilité des entreprises). On pourrait toutefois mentionner que, avec les ERP, on ouvre à l'intérieur de l'entreprise les données, auparavant cloisonnées dans des outils différents, utilisés de façon fermée par des services ou par des métiers. Cela s'entend sous réserve de la question des habilitations dans l'accès aux données, que le système informatique permet de gérer de façon plus ou moins restrictive. Néanmoins, les différentes fonctions spécialisées ne sont plus propriétaires de leurs données autant que cela pouvait être le cas lorsque ces données avaient un format spécifique au sein de l'entreprise, n'étaient disponibles que dans des outils localisés et n'étaient interprétables que par les seuls représentants du métier.

\section{Affirmation des individus et montée du politique}

Ces dernières hypothèses s'entremêlent avec celles, proposées par Konstantinos Chatzis dans l'introduction de cet ouvrage, d'un retour d'une visibilité du politique associé à une place croissante des individus.

Denis Segrestin (2004) rappelle qu'à l'origine des ERP il y avait une double utopie : d'une part un projet de rationalisation par la centralisation, laissant peu de place aux individus et d'autre part un projet plus décentralisé d'ouverture d'opportunité de transversalités. De la même manière, on peut rappeler les liens entre le lean et les démarches qualité dont un des volets (autour des cercles de qualité) était l'écoute et la responsabilisation des agents d'exécution. Les avis sont partagés sur l'ouverture potentielle de ces assemblages. Pour Denis Segrestin (2004), les observations empiriques convergent pour montrer la domination de l'option centralisatrice associée à ces deux types d'assemblages et, comme le notent Gilbert et Leclair (2004), l'étonnante continuité avec les orientations originelles du taylorisme qui reviennent sur l'argument de l'invisibilité des outils de gestion. Cette analyse est contestée par Magali Nonjon et Guillaume Marrel (2015). Linterrogation sur la 
pertinence de l'invisibilité des outils de gestion est explicitement abordée dans leur article sur les architectures informatiques. Ces auteurs relisent en effet l'article classique de Berry sur les « technologies invisibles » à propos des outils de gestion pour souligner par contraste comment aujourd'hui ces technologies sont de plus en plus l'objet de débats. Ces outils " n'existent pas malgré les acteurs, mais sont produits et négociés par eux [...]. Les architectures informatiques de gestion ne sont pas invisibles. Elles n'échappent pas à la volonté des hommes qui les utilisent, les négocient et se les approprient pour restructurer les services et redéfinir les architectures organisationnelles. Les ossatures ne sont pas masquées ou cachées derrière les façades. Elles sont bien visibles aux acteurs qui s'en emparent comme des espaces de lutte, les utilisent dans des stratégies de légitimation personnelles et professionnelles. » La part de l'individu existe, mais on peut se demander si elle ne reste pas confinée aux dimensions micro des résistances locales.

Il faut alors sûrement chercher d'autres assemblages pour voir émerger moins marginalement les individus. Si on prend l'hypothèse de la montée de l'individu et l'exemple des automobilistes qui, à côté de l'offre du système d'information centralisée, ont contribué à développer une offre parallèle à partir des GPS, on peut ainsi noter qu’à côté des assemblages d'outils de gestion évoqués, qui surplombent les employés, d'autres outils apparaissent comme des ressources saisies par les employés. Cela peut être en particulier des outils de communication comme l'ensemble des petits instruments offerts par Google pour organiser une réunion, travailler à plusieurs sur un document. De tels outils viennent compléter ou contourner ceux offerts dans les systèmes centralisés, même si les directions de systèmes d'information sont souvent inquiètes de ce mouvement du « Bring Your Own Device ». On peut aussi imaginer des formes articulées d'outils collaboratifs laissant une place importante aux individus dans des organisations par projet ou de petites organisations innovantes de type start-up ou de collectifs autour d'espace de coworking qui seraient des « assemblages » faisant le pendant aux modèles très centralisés du lean ou des ERP.

Il est aussi nécessaire dans ces comparaisons de s'interroger sur le statut de l'individu et la nature du politique. Les hypothèses proposées par Kostas Chatzis et la plupart des contributions à cet ouvrage portent sur des infrastructures souvent propriété du public et support de services 
à la population. Le contexte politique est celui de la démocratie locale et les individus sont des clients/usagers potentiellement citoyens. Dans le cas de l'entreprise, le contexte est différent, les individus en question sont des salariés dont le contrat de travail inclut la subordination et les dirigeants et propriétaires n'en sont pas élus par les employés. Ce qui se joue d'autonomisation des individus et de rapport de force au sein de l'entreprise n'est ainsi pas tout à fait comparable à ce qui se joue autour de la prestation de services publics.

\section{Conclusion}

Ainsi, que l'on compare les assemblages d'outils de gestion à des infrastructures de la connaissance comme l'Internet ou le système multidimensionnel de la météo, ou à des infrastructures plus matérielles comme un système de production et de transport d'électricité, les mises en équivalences permettent de saisir tout ce qu'il y a de dynamique instable, de modularité dans ces assemblages qui sont plus proches du rhizome que de l'architecture pensée en un seul plan. La confrontation à propos des dynamiques de développement attire l'attention sur les échafaudages et le travail d'individus singuliers qui ont été nécessaires à la production d'ensembles qui semblent aujourd'hui se tenir par euxmêmes. L'interrogation sur les rapports sociaux et politiques en arrièreplan de l'usage de ces infrastructures de la connaissance révélerait là aussi des parallèles frappants, même si les relations sociales entre employeurs et salariés et entre offreurs et utilisateurs de services (en particulier publics) ne sont pas de même nature. Si certaines questions suscitées par le parti pris de confronter les assemblages de gestion à la théorie de l'architecture rejoignent des questions classiques de la sociologie des outils de gestion (en particulier celles concernant la redistribution des pouvoirs associés à leur usage ou les approches micro), d'autres apparaissent plus originales. L'intérêt de la démarche serait aussi d'aller plus avant dans l'exploration d'assemblages moins « architecturés » et plus composites que les ERP.

\section{Références}

Abrahamson E. (1991), "Management Fads and Fashions: The Diffusion and Rejection of Innovations ", vol. 16, n 3, p. 586-612. 
Boussard V. (2001), "Quand les règles s'incarnent. L'exemple des indicateurs prégnants ", Sociologie du travail, vol. 43, n 4, p. 533-551.

Bowker G. C., Star S. L. (1999), Sorting Things Out, New Baskerville (Mass.), The MIT Press.

Chatzis K. (1999), "Searching for standards: French engineers and time and motion studies of industrial operations in the 1950s ", History and Technology, vol. 15, n³, p. 223-261.

Chiapello È., Gilbert P. (2013), Sociologie des outils de gestion, Paris, La Découverte.

Crague G. (2014), Entreprise, management et territoire : le management des implantations territoriales à l'ère de l'entreprise-réseau, Québec, Presses de l'Université Laval.

Edwards P. N., Bowker G. C., Jackson S. J., Williams R. (2009), "Introduction: An Agenda for Infrastructure Studies ", Journal of the Association for Information Systems, vol. 10, numéro spécial, p. 364-374.

Edwards P. N., Jackson S. J., Chalmers M. K., Bowker G. C., Borgman C. L., Ribes D., Burton M., Calvert S., (2012), Knowledge Infrastructures: Intellectual Frameworks and Research Challenges, Report of a workshop sponsored by the National Science Foundation and the Sloan Foundation, University of Michigan School of Information, 25-28 May.

Gilbert P., Leclair P. (2004), "Les systèmes de gestion intégrés : une modernité en trompe-l'œil ? ", Sciences de la société, n 61, p. 17-32.

Greenan N., Guillemot D., Kocoglu Y. (éds) (2010), "Informatisation et changements organisationnels dans les entreprises ", Réseaux, $\mathrm{n}^{\circ} 162$, p. 9-304.

Greenan N., Hamon-Cholet S., Ughetto P. (éds) (2016), Salariés du public, salariés du privé face aux changements, Paris, L'Harmattan.

Greenan N., Walkowiak E. (2010), "La dynamique des changements à long terme ", Réseaux, n¹62, p. 231-274.

Hughes T. P. (1993), Networks of power: electrification in western society, 1880-1930, London-Baltimore, Johns University Press.

Jeannot G., Guillemot D. (2010), «Gérer par les outils ou par les hommes? Un bilan quantitatif de la "modernisation de la gestion" de l'État ", Politiques et management public, vol. 27, $\mathrm{n}^{\circ}$ 4, p. 73-102. 
Kocoglu Y., Moatty F. (2010), «Diffusion et combinaison des TIC. Les réseaux, la gestion des données et l'intégration par les ERP ", Réseaux, $\mathrm{n}^{\circ} 162$, p. 37-72.

Kocoglu Y., Moatty F. (2012), "Les ERP entre mythe et réalités : les stratégies d'intégration fonctionnelle des entreprises françaises en 2006 ", Document du travail du CEE, $\mathrm{n}^{\circ} 157,29 \mathrm{p}$.

Nonjon M., Marrel G. (2015), « Gouverner par les architectures informatiques. Logiciels et progiciels de gestion intégrée dans le secteur social ", Gouvernement et action publique, $\mathrm{n}^{\circ}$ 2, p. 9-24.

Orlikowski W. J. (1992), "The Duality of Technology: Rethinking the Concept of Technology in Organizations ", Organization Science, vol. 3, $\mathrm{n}^{\circ} 3$, p. 419-420.

Segrestin D. (2004), «Les ERP entre le retour à l'ordre et l'invention du possible ", Sciences de la société, n 61, p. 3-16.

Shimizu K. (1999), Le toyotisme, Paris, La Découverte.

Ughetto P. (2009), "Une réorganisation au concret : l'implantation du lean manufacturing comme travail managérial ", communication aux $\mathrm{XII}^{\mathrm{es}}$ Journées de sociologie du travail, Nancy, 25-26 juin, $13 \mathrm{p}$.

Weick K. E. (1995), Sensemaking in Organizations, Thousand Oaks (Calif.), Sage. 



\title{
Les plateformes d'innovation, de marque, de conseil
}

\section{Vers une infrastructure numérique de la relation-client?}

\author{
Émile Gayoso \\ Thomas Jammet \\ Nicolas Klein
}

Dans la gamme des relations que l'essor d'Internet et des TIC transforme en profondeur, celles liant les individus aux entreprises ont reçu moins d'attention de la part des sciences sociales que les communications interpersonnelles ou la participation citoyenne aux institutions politiques. Pourtant, depuis que les barrières entre producteurs et consommateurs de contenu se sont vues abaissées par les évolutions techniques d'Internet (Cardon, 2010, 2011) - lesquelles sont souvent résumées sous le vocable de Web 2.0 -, depuis que les acteurs marchands ont massivement investi le réseau des réseaux, dessinant les contours d'une " économie de l'attention " (Kessous et al., 2010) où l'interaction entre une marque et un internaute, et la captation de celui$\mathrm{ci}^{1}$, représente l'unité élémentaire de la valeur marchande, les occasions et les formats de la relation de l'individu à l'entreprise se sont multipliés.

D'abord cantonnée au commerce électronique, aux sites web institutionnels et à la publicité, cette relation s'est étendue aux services de messagerie personnelle (par le biais des newsletters et de l'intégration de la prise en charge des demandes des clients par courriers électroniques

"L'enjeu central de l'économie de l'attention porte sur la tension à laquelle s'exposent les acteurs de l'Internet et des médias électroniques. Celle-ci oppose deux logiques. La première vise à protéger l'attention de la surcharge d'information et à optimiser son allocation, la seconde a pour objectif de la capter dans une perspective de profit" (Kessous et al., 2010, p. 365). 
(Licoppe, 2002)), aux blogs, wikis et autres outils rédactionnels collaboratifs (que ce soit par la création de blogs d'entreprise ou par la relation avec des "blogueurs influents "), aux sites de réseaux sociaux (où des espaces institutionnels sont créés par les entreprises, puis enrichis et maintenus par des équipes de community managers, internes ou externes) ainsi qu’à de nombreux dispositifs ad hoc créés par les entreprises elles-mêmes. Ces dispositifs, qui sont souvent nommés plateformes, forums, ou encore écosystèmes, permettent aux entreprises d'établir avec les internautes, qu'ils soient clients ou " prospects " ${ }^{2}$, des canaux de communication parfois inédits, susceptibles aussi de servir à des formes d'encadrement et de contrôle de la parole des consommateurs (Benedetto-Meyer et Raimond, 2011). Ils participent de pratiques de marketing apparues dans les années 1990 comme le "marketing expérientiel ", le " marketing relationnel » et, depuis l'essor du Web 2.0, le «marketing collaboratif» (Cova et Cova, 2009).

Plus profondément, les plateformes numériques de relation-client s'inscrivent dans cette transformation concomitante des discours managériaux et de l'organisation des entreprises qu'on peut qualifier d' « orientation-client " et qui s'est déployée depuis les années 1970 dans un contexte entremêlé d'intensification de la concurrence, d'informatisation des entreprises et d'essor d'un fonctionnement en réseaux (Caron, 2010), sans qu'aucun rapport univoque de cause à effet puisse être établi entre ces différents phénomènes. Pierre-Jean Benghozi a bien montré les effets contradictoires de cette nouvelle orientation des entreprises vers le client, qui d'un côté consacre ce dernier dans les processus organisationnels (de la conception à la vente) et vient remplacer une conception de l'entreprise par " métiers ", et de l'autre, en rationalisant l'approche du client et en standardisant les offres par segments de clientèle, procède à une sorte d'éloignement de l'organisation vis-à-vis des singularités de ce client. On assiste ainsi à un "double mouvement de standardisation et de "customization", analogue à celui qui fait passer du sur-mesure au prêt-à-porter » (Benghozi, 1998).

Terme issu du monde commercial et d'usage courant aujourd'hui dans les entreprises pour désigner un client potentiel. 
Ce qu'on appelle la gestion de la relation-client (GRC) ou plus communément CRM (Customer Relationship Management) vient d'ailleurs poursuivre ce mouvement de rapprochement et de normalisation (Lefebure et Venturi, 2000) à la faveur de l'essor parallèle, depuis la fin des années 1990, des centres d'appel, des serveurs vocaux interactifs et du Web, qui sont autant de canaux d'interaction permettant aux entreprises d'enregistrer de nombreuses données et de faire de ces traces la matière première d'une nouvelle connaissance-client, fondée sur l'outil statistique (Benedetto-Meyer, 2011).

Mais ce mouvement ne se réduit pas à un nouvel outillage des services commerciaux des entreprises. Si les instruments jouent un rôle important dans l'essor d'un "CRM 2.0 " (Benedetto-Meyer et Raimond, 2011), il faut souligner le rôle joué par de nouveaux acteurs tels les community managers et les webconseillers qui portent de nouvelles pratiques relationnelles bien au-delà de la fonction de vente. Ainsi, les webconseillers sont capables d'intervenir hors du champ formalisé de l'interaction commerciale et de son attente inquiète du "rebond $»^{3}$ (Licoppe, 2006) afin d'établir des relations complexes et non directement marchandes avec les internautes, en mobilisant envers ces derniers différents registres de communication. De même, les postes de community managers fleurissent, qu'ils soient consacrés à l'e-réputation de l'entreprise, c'est-à-dire à la gestion de son image de marque sur Internet, ou qu'ils aient pour tâche d'aider à la collaboration de chefs de projet et de produits avec des développeurs, des designers ou de simples utilisateurs pour inclure une "vision client " dans le développement d'une innovation ${ }^{4}$. Ces formes de relations non directement marchandes, couplées aux actions de recueil de la connaissance client, inscrivent la

3 Christian Licoppe définit ainsi le moment où le conseiller bénéficie de suffisamment d'informations contextuelles sur le client pour tenter de réorienter l'interaction en l'inscrivant dans un engagement contractuel.

4 En ce qui concerne le domaine de l'innovation, le groupe Orange a ainsi mis en place depuis 2004 deux plateformes de bêta-tests de produits et services en cours de développement (Labo et Oniro, désignés ici et dans la suite du texte sous un nom d'emprunt), une plateforme d'ouverture des API (Applications Programing Interface) pour les développeurs indépendants et les start-up (Orange Partner), une plateforme d'information et de communication autour des nouvelles technologies (Le Collectif), un blog (live Orange blog) et une télévision en ligne (Orangeinnovation Tv), l'ensemble de ces dispositifs étant accessibles depuis une interface de téléchargement d'applications pour mobiles et tablettes (Orange Labs prime zone). 
relation-client numérique dans le registre des opérations de captation, dont Cochoy a montré qu' elles étaient au cœur du travail relationnel des entreprises dans la société (Cochoy, 2004, 2008).

Cette multiplication des dispositifs en ligne et des acteurs de la relation-client pose la question de leur articulation. Y a-t-il quant aux rôles joués par ces plateformes, quant aux compétences requises pour les animer, quant aux relations se nouant entre leurs usagers et les porte-parole des entreprises, des logiques communes que l'on pourrait déceler ? Pour le dire autrement, a-t-on affaire à un archipel de dispositifs relativement indépendants dans leur rattachement fonctionnel à l'entreprise (le marketing, la vente, la communication, l'innovation) comme dans leur fonctionnement technique (les choix logiciels en termes de content management et de community management) et organisationnel (la composition des équipes dédiées à ces dispositifs et les compétences définies pour ces métiers ou fonctions), ou bien s'oriente-t-on vers une infrastructure numérique de la relation-client ?

Pour répondre à cette question, nous nous appuierons sur la construction du concept d' " infrastructures informationnelles " au sein du champ des STS par Susan Leigh Star et Karen Ruhleder (Star et Ruhleder, 1996) puis Geoffrey Bowker, lequel en propose, dans une analyse du système international de classification des maladies (ICD), une caractérisation en cinq volets (Bowker et Noyer, 1997) :

- « elle s’inscrit [...] dans un système enchevêtré et distribué d'autres structures ;

- elle est transparente ("elle n'a pas à être réinventée à chaque fois") ;

- elle est étendue et transversale ;

- elle a une dimension essentiellement collective ;

- enfin elle repose sur un ensemble de conventions et pratiques concrètes dont elle est à la fois l'expression et l'exprimé. "

Si le concept d'infrastructure informationnelle nous servira de point d'appui dans la discussion de la dynamique de généralisation et de standardisation de dispositifs et de pratiques en ligne de relationclient, c'est bien à l'examen précis et comparé de tels dispositifs que ce chapitre sera consacré et non à un approfondissement théorique de la 
notion. S'appuyant sur trois travaux de thèse $\mathrm{e}^{5}$ portant respectivement sur les plateformes numériques d'innovation, de conseil et de marque, notre contribution cherche davantage à pointer un processus d' " infrastructuralisation ${ }^{6}$ qu’à caractériser une infrastructure informationnelle bien établie de l'extension en ligne du lien entreprise-individu.

\section{Les nouvelles frontières de la relation-client : acteurs et dispositifs}

S'il est difficile de trouver une lisibilité à l'ensemble disparate de dispositifs que les entreprises déploient en ligne pour informer, orienter, séduire, capter, fidéliser les internautes, c'est que l'environnement d'Internet est à la fois une contrainte et une ressource pour les organisations. Ces dernières doivent d'un côté construire des plateformes ad hoc leur permettant d'étendre sur la Toile leurs processus et leurs savoir-faire, et de l'autre répondre aux développements propres du Web qui, en consacrant certains intermédiaires ${ }^{7}$, les contraignent à s'insérer dans ce milieu étranger où les sites de réseaux sociaux, les forums et les blogs portent de nouveaux formats relationnels qui s'imposent aux acteurs de l'entreprise. Pourtant, derrière ce poudroiement de dispositifs singuliers, on observe une nette convergence de l'architecture logicielle

5 Cette étude s'appuie sur trois enquêtes prenant toutes pour objet de très grandes entreprises, pour la plupart françaises, dans le secteur des télécommunications, du transport de voyageurs, de la banque et de l'assurance. Les trois enquêteurs ont procédé à des entretiens semi-directifs auprès de community managers de marque ou d'innovation, de responsables de plateformes numériques et de webconseillers. Le cas d'Orange, commun à ces trois enquêtes, sera particulièrement mobilisé, mais les phénomènes décrits ont une validité plus générale dans les grandes entreprises de services.

6 Nous emploierons ce néologisme, utilisé dans les travaux de STS consacrés aux infrastructures, dans le sens que lui confère Alexandre Mathieu-Fritz dans sa contribution au présent ouvrage portant sur les dispositifs de télémédecine. Ce dernier propose de désigner par ce terme "le processus de développement de vastes dispositifs sociotechniques présentant, suivant des niveaux d'achèvement plus ou moins élevés, certains traits caractéristiques des infrastructures ".

7 Parmi lesquels figurent les géants du Web bien sûr, tels Google, Facebook, Twitter, Microsoft ou Amazon, mais aussi les forums, les blogs, les wikis qui servent aux amateurs, aux usagers, aux clients à s'exprimer et à se rassembler autour de pratiques, de produits ou de services qui concernent parfois directement les marques. 
des plateformes de relation-client. Schématiquement, on peut dire que celle-ci s'effectue par la fusion d'outils de type forums (permettant une gestion thématique et chronologique des contributions et relevant du content management) et d'outils de médias sociaux (permettant la construction d'un profil personnalisé ainsi que la gestion d'un panel de relations privilégiées ${ }^{8}$ et relevant du community management $\left.{ }^{9}\right)$.

S’il n'y a donc pas une forme unique de plateforme numérique de relation-client, il y a bien une cohérence quant à leur architecture logicielle. Par ailleurs, parce que la relation-client numérique se déploie en partie dans un environnement que l'entreprise ne contrôle pas (forums non officiels de marques, pages Facebook dont les métriques peuvent changer du jour au lendemain, etc.), elle répond bien au premier critère de Bowker qui veut qu'une infrastructure informationnelle se place dans un enchevêtrement de structures ${ }^{10}$.

Le quatrième critère, qui veut qu'une infrastructure informationnelle ait "une dimension essentiellement collective ", est également rempli dans le cas des plateformes numériques de relation-client, sans qu'il soit besoin pour l'affirmer de mener une enquête approfondie. En effet, les plateformes s'adressent à des collectifs d'internautes, clients ou prospects, qui se les approprient et contribuent à les structurer de telle sorte que le contenu disponible sur les plateformes est largement produit par les usagers (les commentaires, les posts, les $\mathrm{FAQ}^{11}$, les photos, les statuts publiés). Les dispositifs numériques de relation-client s'insèrent à ce

8 Les deux médias sociaux de référence étant Facebook et Twitter, proposant respectivement des systèmes de mise en relation d'amis et de suiveurs fans et followers.

9 L'aspect forum ou média social peut être plus ou moins développé selon la fonction de la plateforme considérée. Ainsi, le community management de marque sera davantage associé aux médias sociaux et l'activité de conseil en ligne aux forums. Le community management de la co-innovation, lui, s'opère sur des dispositifs singuliers qui mêlent ces deux types d'outils, en donnant, au fur et à mesure des mises à jour des sites web, de plus en plus d'importance à la dimension sociale de la plateforme. Dans leur article séminal, Star et Ruhleder parlent d'un enchevêtrement dans d'autres "structures, arrangements sociaux et technologies" ("structures, social arrangements and technologies»), ce qui est bien le cas ici puisque les sites de réseaux sociaux et les forums sont à la fois supportés par des technologies informatiques spécifiques et supportent des pratiques relationnelles distinctes. 
titre également dans cette évolution d'Internet qualifiée de "Web 2.0 ", qui donne une grande visibilité aux contenus générés par les utilisateurs (user generated content) (Coutant et Stenger, 2012).

Le deuxième critère, la «transparence à l'usage » des infrastructures informationnelles, qui fait que leur agencement sociotechnique n'a pas à " être réinventé à chaque tâche, mais supporte de manière invisible ces tâches " (Star et Ruhleder, 1996), est un peu plus problématique. D’un côté, sur le plan technique, cette transparence est une caractéristique de l'environnement informatique d'Internet, dont une des fonctions principales est précisément de rendre relativement indépendant des acteurs humains l'accès à des données stockées sur des serveurs qui répondent automatiquement aux requêtes (clics) des internautes. Mais cette transparence est certainement moins complète en ce qui concerne le volet social de l'agencement sociotechnique de la plateforme. En effet, au-delà de simples clics, les internautes formulent des requêtes écrites, ce qui suppose une identification préalable et une compréhension des rôles des webconseillers et des community managers chargés d'y répondre. Or ces métiers étant encore peu connus et leurs prérogatives pas encore stabilisées, de nombreuses interactions avec des clients néophytes doivent d'abord définir un cadre à l'échange avant qu'une demande ne puisse être formulée et traitée. Le critère de transparence n'est donc que partiellement rempli, ce qui nous incite à parler d'" infrastructuralisation » au sujet de la relation-client numérique plutôt que d'une infrastructure aboutie.

Restent donc les troisième et cinquième critères retenus par Bowker : l'extension et la transversalité du dispositif d'une part et l'existence de conventions et pratiques concrètes dont il soit le support d'autre part. Nous montrerons dans la suite de cette partie que ces nouveaux postes-frontières de l'entreprise que sont les plateformes numériques de relation-client se déploient de manière transversale aux différentes fonctions organisationnelles. La partie suivante sera consacrée quant à elle aux nouvelles conventions relationnelles entre porte-parole des entreprises et internautes qui s'observent sur les dispositifs en ligne de relation-client. 


\section{L'essor du community management...}

\section{... de marque}

Activité d'intermédiation numérique en plein essor en France depuis 2009-2010, le community management apparaît comme une modalité inédite de promotion des marques et de gestion de la relation-client au moyen des plateformes de conversation et d'échange de contenu regroupées sous l'appellation de sites de réseaux sociaux. L'une des principales difficultés de l'étude de cet espace de pratiques consiste en l'extrême diversité des trajectoires, des profils et des intitulés de poste des personnes se réclamant de cette activité, ainsi qu'en l'évolution incessante des outils qu'il mobilise, au premier rang desquels Facebook et Twitter. Le community management de marque est appréhendé ici comme la fonction des personnes chargées de représenter une marque ou un produit sur les sites de médias sociaux, et de susciter, au moyen d'un dispositif de captation interactif et conversationnel, une attitude de "sympathie agissante " des internautes à l'égard de l'organisation incarnée. La notion de "sympathie agissante ", empruntée à Thierry Viale (1997), vise à distinguer le travail de relations publiques (RP) de celui de la publicité commerciale, en insistant sur la dimension humaine et relationnelle, en un mot "sociale " de la communication institutionnelle. Elle s'avère particulièrement intéressante à appliquer à la fonction de community management, qui s'efforce de se distinguer de la publicité tout en se voyant contrainte de mobiliser ses indicateurs de performance pour l'évaluation de son activité. L'identité professionnelle en construction des community managers insiste ainsi beaucoup, dans sa mise en récit, sur sa singularité vis-à-vis du marketing traditionnel et en particulier sur son rejet d'une communication « descendante " (topdown).

« Pourquoi est-ce que les sites de réseaux sociaux aujourd'hui fonctionnent et pourquoi est-ce qu'ils fonctionnent dans notre rapport à la marque, c'est tout simplement du rejet. C'est un rejet massif du marketing traditionnel, un rejet massif de la publicité. Pas nécessairement tant un rejet violent qu'un désintéressement, une espèce de désenchantement " (Consultant Social Media, cabinet-conseil en intelligence digitale). 
Explicitement orienté vers le client, saisi au prisme des figures du fan et du follower, le community management de marque prend appui sur les préceptes du marketing relationnel (Cochoy, 2008), expérientiel (Hetzel, 2002 ; Carù et Cova, 2006) et tribal (Cova et Cova, 2002), et se caractérise par un faisceau de pratiques orientées vers la consolidation de l'image de marque, de sa notoriété, en un mot de sa e-réputation.

\section{... d'innovation}

Dans un contexte d'économie de la connaissance (Foray, 2009) où la capture et la mise en forme de l'information sont devenues des compétences cruciales des organisations, le " régime d'innovation intensive $»^{12}$ (Hatchuel et Weil, 1995, 1999) ayant émergé à la fin des années 1970 se trouve renforcé. Face aux pressions accrues de la concurrence et à la mise en réseau de la "fonction recherche » de l'entreprise (Caron, 2010), la R\&D se transforme et cherche de plus en plus à trouver à l'extérieur des savoirs et savoir-faire qu'elle n'est plus en mesure de produire seule. Dès lors, les sources externes d'innovation pour une entreprise sont de deux types : les autres organisations (publiques ou privées, grandes entreprises, PME ou start-up, associations, etc.) et les individus, isolés ou rassemblés en collectifs sans structure légale (forums d'amateurs par exemple).

L'information que recherchent les innovateurs est souvent difficile à obtenir, soit parce qu'elle est d'une grande complexité et que sa construction nécessite un investissement cognitif important, soit parce qu'elle " colle» (von Hippel, 1994) aux représentations de son détenteur. Fondées d'une part sur l'observation de la créativité exceptionnelle de certains individus : les utilisateurs-pilotes (lead-users), définis et étudiés comme tels par Eric von Hippel (von Hippel, 1986), et d'autre part sur les possibilités nouvelles de collaboration en ligne fournies par

12 Régime économique dans lequel tous les modes de formation de la valeur sont soumis à une logique d'innovation. Ces modes de formation de la valeur sont divers et ne se réduisent pas à la technologie. Comme le souligne l'inventeur de ce concept dans un article sur les " organisations orientées conception " (Hatchuel et al., 2002), « les entreprises agissent sur des marchés où l'innovation peut porter indifféremment sur les qualités fonctionnelles ou communicationnelles d'un produit, son style, les services qui l'accompagnent, les valeurs humanitaires, environnementales qu'il exprime ou respecte, etc. ". 
Internet, les plateformes de co-innovation avec l'usager constituent un phénomène émergent. Un community management de l'innovation s'est donc mis en place au fur et à mesure que de grandes entreprises élaboraient leurs propres plateformes ${ }^{13}$. Ce community management a un caractère spécifique à l'activité d'innovation, puisqu'il tâche de mobiliser l'" intelligence collective " des internautes, de "décoller " l'information dont ces derniers sont porteurs en l'extrayant des contextes spécifiques liés à leurs usages. Pour cela, deux outils très simples sont souvent mobilisés par les community managers : la relance ("décoller " l'information en demandant à un internaute un approfondissement de sa contribution précédente) et la mise en lien avec les autres participants (pour mettre en branle l' "intelligence collective») :

\section{Figure 1. Capture d'écran d'une relance adressée lors d'un bêta-test par Rosario, community manager de Oniro (Orange) à l'un des membres de la plateforme : Nico35}

\section{Rosario, modérateur}

Nico35, Marjorie rapporte une solution me semble-t-il... Je souhaiterais pouvoir expliquer de manière simple la manière dont on range ses favoris. Pour ce qui est du partage, comment présentez-vous les url ? "tiens regarde là, c'est intéressant" ou en dites-vous davantage? Si vous souhaitez donner des exemples, sentez-vous libres de le faire !

Mais une part significative des prérogatives du community management de la co-innovation est commune avec celles prévalant pour la gestion d'une "communauté » de fans. Ainsi, comme les community managers de marque ouvrant sur les sites de réseaux sociaux, les community managers attachés aux plateformes d'innovation obéissent à des contraintes d'image de marque, de rapprochement conversationnel et de connaissance-client (cf. partie II). En outre, de la même façon que les community managers de marque cherchent à se distinguer des acteurs

13 Les plateformes d'innovation ayant fourni la matière de la rédaction de ce chapitre sont Labo et Oniro pour Orange, L'Atelier SFR pour SFR, La Fabrique RATP pour la RATP, Imagine TGV pour la SNCF et Ideastorm pour Dell. On peut distinguer parmi celles-ci deux types de dispositifs : ceux où l'usager est force de proposition et où l'entreprise trie et développe les idées lui venant de l'extérieur (Imagine TGV, Ideastorm), et ceux où c'est l'entreprise qui propose des maquettes de produits et services tandis que l'usager est en position de critique et d'amendeur (Oniro, Labo, l'atelier SFR et La Fabrique RATP) : les premières sont dites plateformes de bêtatest, les secondes d'idéation. 
du marketing traditionnel et de sa culture descendante, les community managers de l'innovation participative situent leur action en rupture avec la culture technique des ingénieurs, une culture qu'ils jugent trop " orientée fonctionnalités » et pas assez « client ", une culture qu'il s'agit de transformer en " mettant à disposition des chefs de produit un outil d'interaction client à chacune des étapes du TTM ${ }^{14}$ " (Responsable de la plateforme Labo, Orange).

\section{L'essor des webconseillers}

Apparaissant dans un premier temps, en 2011, comme un énième canal de relation client, les webconseillers se distinguent de leurs collègues vendeurs (au téléphone ou en boutique) par leurs missions, qui consistent moins à traiter directement un type de demande particulier (réclamation, assistance, prise de commande...) qu’à réceptionner et traduire ces demandes pour orienter les clients vers l'interlocuteur adéquat (avant et après sa souscription aux offres de l'entreprise) ainsi qu'à assurer une continuité dans le suivi du client tout au long du traitement de sa demande par l'entreprise. La position de webconseiller ${ }^{15}$ est celle du premier (et parfois unique) contact du client sur les plateformes numériques. Sa posture peut se décliner en deux étapes de la relation client. Avant la transaction (la souscription du client à une offre), le webconseiller répond à des demandes de renseignements sur les offres et les promotions de l'entreprise, renvoyant le client vers des sources d'informations présentes sur Internet (en lui donnant par exemple des liens vers des pages sur le site de l'entreprise). Après la

14 Pour Time To Market: il s'agit du nom donné au cycle de vie du produit lors de son développement au sein d'Orange.

15 L'activité étudiée ici est celle des webconseillers qui, chez Orange, réceptionnent et répondent aux demandes des clients émanant des forums (forum d'Orange, de Sosh - marque low cost d'Orange lancée suite à l'arrivée de Free sur le marché mobile -, et du forum Orange non officiel) ainsi que des sites de réseaux sociaux (ici Twitter). En parallèle, nous nous intéresserons à l'intervention spécifique, depuis septembre 2013, d'un webconseiller sur le blog des habitants d'un nouveau quartier en région parisienne, pour répondre aux demandes des habitants (pas seulement des clients) et à leurs problématiques (liées au départ à leur emménagement puis sur un champ plus large) sur l'ensemble du faisceau d'intervention de l'entreprise (qu'il s'agisse du domaine commercial ou du domaine technique). 
transaction, le webconseiller prend en charge des demandes d'assistance liées au suivi de la commande ou aux usages, qu'ils soient techniques ou commerciaux.

La posture du webconseiller entre le client et les segments de l'entreprise ainsi que sa double action de traduction, d'orientation et de suivi de la demande du client d'une part, et de représentation de l'entreprise, de son discours commercial et de la posture de service qu'elle veut promouvoir sur les plateformes numériques d'autre part, peut être comprise comme une posture d'interface. Cette posture s'apparente à celle d' " entrepreneur-frontière " développée par Henri Bergeron (2013), les deux jouant sur leur " multivocalité » (sa capacité à interpréter et lier plusieurs langages, ici celui des clients sur les sites de réseaux sociaux, et celui, très segmenté et spécialisé de l'organisation) et leur " multipositionnement ", en usant de leur appartenance à plusieurs mondes sociaux pour défendre leurs intérêts propres (la posture d'interface du webconseiller ${ }^{16}$ ) et ceux de leur entreprise (son résultat commercial).

Enfin, comme les community managers vis-à-vis de la culture descendante des publicitaires ou des ingénieurs, les webconseillers se définissent par opposition à leurs plus proches parents : les vendeurs. Les webconseillers expriment en effet une aversion, non pas à participer à la vente, mais à ce que leur activité soit associée à de la vente (et qu'euxmêmes soient assimilés à des vendeurs, ce qu'ils ont déjà été, pour une grande partie d'entre eux, durant leur parcours professionnel). Ils sont pourtant conscients que leur relation au client a une visée essentiellement commerciale et ne remettent pas cela en cause. En d'autres termes, ils acceptent que leur activité serve à faire vendre, mais pas de vendre euxmêmes. "Après, je ne critique pas : j'avais des collègues à qui cela allait très bien, les challenges, qui se retrouvaient parfaitement là-dedans. Mais pas moi »(Webconseiller Orange).

16 Contrairement au " traducteur » de Michel Callon (1986), l'entrepreneur-frontière, dans sa position d'interface, ne cherche pas à aligner les acteurs (les clients sur les plateformes et les conseillers dans l'organisation) sur une vision commune, mais plutôt à leur permettre de communiquer sans modifier leurs dispositions sociales, à la manière d'un « objet-frontière " (Star et Griesermer, 1989). 
Ainsi, de façon transverse aux différents processus organisationnels, des structures et des acteurs divers se sont établis à l'interface entre l'individu et l'entreprise, en suivant à chaque fois le même mécanisme de différenciation vis-à-vis des fonctions traditionnelles de l'entreprise, critiquées autant pour leur éloignement du client (communication descendante, R\&D cloisonnée) que pour leur exigence de rentabilité (la relation-client pour la vente). Nous allons voir désormais comment ces nouvelles structures et les acteurs qui les animent déploient de nouvelles conventions relationnelles avec les internautes, que l'on peut résumer par l'articulation paradoxale entre captation et familiarité.

\section{Captation et familiarité : de nouvelles conventions pour la relation-client}

\section{La pluralité des formes de la captation}

Dans son étude du travail relationnel des acteurs marchands, Franck Cochoy a porté une attention particulière à "l'ensemble des opérations qui s'efforcent d'avoir prise, d'attirer vers soi, de garder ce ou ceux que l'on a attiré(s) » (Cochoy, 2004), opérations qui relèvent d'une catégorie qu'il propose d'appeler la "captation ». Entendue dans ce sens et appliquée à l'univers marchand, la captation ne se réduit pas au sens spontané qu'on lui confère d'acquisition d'un nouveau client, ou, dans le langage autochtone des entreprises, de conversion d'un prospect en client, mais englobe les opérations de séduction propres aux dispositifs d'attraction tels que les campagnes publicitaires ou le packaging ainsi que l'ensemble des opérations et dispositifs de connaissance-client dont les départements de marketing peuvent avoir l'initiative et dont l'objectif est précisément, en s'appuyant sur l'analyse statistique des traces relatives au client (Benedetto-Meyer, 2011) d'" avoir prise" sur eux, en les segmentant plus finement, en connaissant mieux leurs pratiques, leurs goûts et leurs désirs.

La comparaison de plusieurs plateformes de bêta-tests de très grandes entreprises montre au contraire à quel point les enjeux de connaissanceclient sont cruciaux, prenant d'ailleurs toujours plus d'importance au fur et à mesure que les plateformes gagnent en nombre d'inscrits : 
«Un des moteurs, quelque part de Labo, c'est l'avant-première, c'est le test, c'est donner son avis, mais c'est de le faire de manière sympathique, donc en fait il y a tout un travail qui est intéressant sur l'ergonomie du Web, l'animation et la capacité à capter aussi des "insights clients", donc ça, c'est un travail qu'on a en cours; donc toujours pour rester aussi modernes, donc là on garde notre philosophie communautaire, par contre, il faut être clair : aujourd'hui, au niveau du panel, là je serai bientôt à 50000 clients, qualifiés, quantifiés, si tu veux aujourd'hui, c'est une communauté de la taille d'un panel ! C'est géré comme une communauté, mais en fait ça prend des formes de panel " (Responsable de la plateforme Labo, Orange).

On observe ainsi sur les plateformes de co-innovation les plus volumineuses (Ideastorm de Dell, Labo d'Orange et l'Atelier SFR de SFR) une tendance des entreprises à s'intéresser de plus en plus aux contributions des internautes non pour ce qu'elles valent comme "bonne idée ", comme information sur les usages, mais pour ce qu'elles valent relativement à un segment de marché, comme information sur le client. Ce glissement de l'usager au client dont les plateformes de co-innovation sont le théâtre consacre leur rôle dans le processus marketing de captation (de connaissance-client). Celle-ci s'incarne par le recours de plus en plus fréquent des plateformes d'innovation participative à des sondages et autres formes de questionnaires qui, étant donné le volume de la base de clients, peuvent être assimilés par les chefs de produit en bénéficiant à des études de marché, tout en étant beaucoup plus rapides à mettre en place et d'un coût deux à trois fois moindre.

Les plateformes de conseil et de marque, quant à elles, même si elles se définissent par opposition à la vente et à la publicité, ont un rôle très clair dans la démarche de captation des entreprises : elles servent a minima à établir une relation de sympathie et de confiance entre une marque et un individu, et au mieux à faire de ce dernier un " ambassadeur de la marque ". Le community manager doit ainsi savoir tirer profit de l'expressivité des internautes pour en faire les porteurs et prescripteurs du discours promotionnel de l'entreprise :

"On fait une différence entre des simples fans ou followers, et ce qu'on peut appeler des ambassadeurs de marque, ce qui correspond davantage à ce qu'on pourrait appeler une communauté, c'est-à-dire un système de 
porte-parole où on va identifier des personnes qui vont être très sensibles à notre discours, qui vont souvent être en affinité, bien apprécier la marque [...] et qui vont systématiquement la défendre " (Consultante influence, agence).

Du point de vue des acteurs, cette démarche est facilitée par la spontanéité et la dimension publique qui caractérisent les usages du Web social, suivant l'idée que les remerciements, au même titre que les plaintes, sont susceptibles d'influencer les autres internautes qui les voient :

"L'objectif, c'est comment rendre un client moins mécontent, voire content, voire un ambassadeur quand on a super bien réussi sa relation client. Ça marche quand la personne revient et que spontanément elle dit quelque chose de bien sur nous. Ça, c'est génial, hein ! Ça arrive avec les sites de réseaux sociaux. Par contre, c'est très rare qu'on vous rappelle sur la hotline en disant : "Ah je suis très content quand même, en fait." Ça, ça n’arrive jamais [Rire] " (Responsable communautés digitales, société de transports).

Pour les webconseillers, l'inscription dans la stratégie commerciale est à rechercher en amont de la transaction. Pour eux, l'absence de vente apparaît comme une condition d'établissement d'une relation avec les clients sur les plateformes numériques. Cela peut relever d'un accord implicite entre clients et webconseillers ou être beaucoup plus explicite : dans le cas du blog, l'absence de vente et de démarchage a fait l'objet d'un accord préalable avec les administrateurs et a été inscrite dans une charte que le premier conseiller à y être intervenu a diffusée sur le fil de discussion : "Ça paraîtrait étrange, je pense... Enfin, c'est implicite qu'on n'est pas là pour ça. C'est aux clients d'en faire la demande après..." (Webconseiller, Orange).

L'action des webconseillers sur les plateformes numériques s'inscrit bien dans ce que Franck Cochoy nous montre du marketing relationnel et des dispositifs pouvant générer et gérer du lien social, ou encore, par le biais de la relation, faire évoluer les dispositions des clients, les amener à transformer leurs comportements (Cochoy, 2008). Sur les plateformes, les webconseillers sont les plus à même d'identifier et de qualifier les "états du client " (Kessous et al., 2014), permettant par la suite à leurs collègues vendeurs de construire leur argumentaire de vente. 
Par ailleurs, les webconseillers établissent une continuité de relation, affichent une posture de service et, surtout, de désintéressement, afin de construire un lien de confiance avec le client susceptible de l'amener - et c'est le pari de l'entreprise - à faire lui-même une demande de prise en charge commerciale. Ici encore, le blog permet d'illustrer cette posture :

"Là où c'est difficile, les premières semaines, il ne fallait surtout pas que j'aille pousser de l'information commerciale. Maintenant, on peut plus le faire, ça y est, on est connus... Ils se disent : "S'ils nous en parlent, c'est que c'est intéressant." On sent bien qu'ils l'acceptent » (Premier webconseiller à être intervenu sur le blog, Orange).

Mais pour créer une relation de confiance avec les internautes, pour les séduire et s'adapter à leurs façons d'être et de parler, le discours normalisé de la grande organisation aux processus rationalisés trouve vite ses limites. C'est pourquoi les acteurs aux avant-postes de la relation-client numérique se doivent de mettre en place une autre forme de relation à la personne, plus à même d'aller au plus près de son intimité, de ses préoccupations singulières. Community managers et webconseillers s'engagent alors dans un régime familier de relationclient.

\section{Vers un client familier}

Parce que le Web rend publics des discours dont le registre était caractéristique de la sphère privée (Cardon, 2010), ses usages par les clients connectés poussent les organisations à personnaliser davantage leurs relations publiques. Prenons le cas d'Orange. Sur Twitter, les webconseillers possèdent des pseudos similaires à ceux utilisés par les clients tandis que, sur le principal forum non officiel de la marque, ils adoptent un profil similaire à celui de n'importe quel autre utilisateur. Sur la plateforme de co-innovation Labo, il arrive que les community managers souhaitent leur anniversaire aux membres inscrits ou qu'ils échangent avec les bêta-testeurs le dimanche, depuis leur domicile. Et voici comment un community manager de la page Facebook d'Orange France répond à une plainte exprimée dans un registre humoristique et affectif : 
Figure 2. Exemple de réponse personnalisée à la plainte d'un internaute

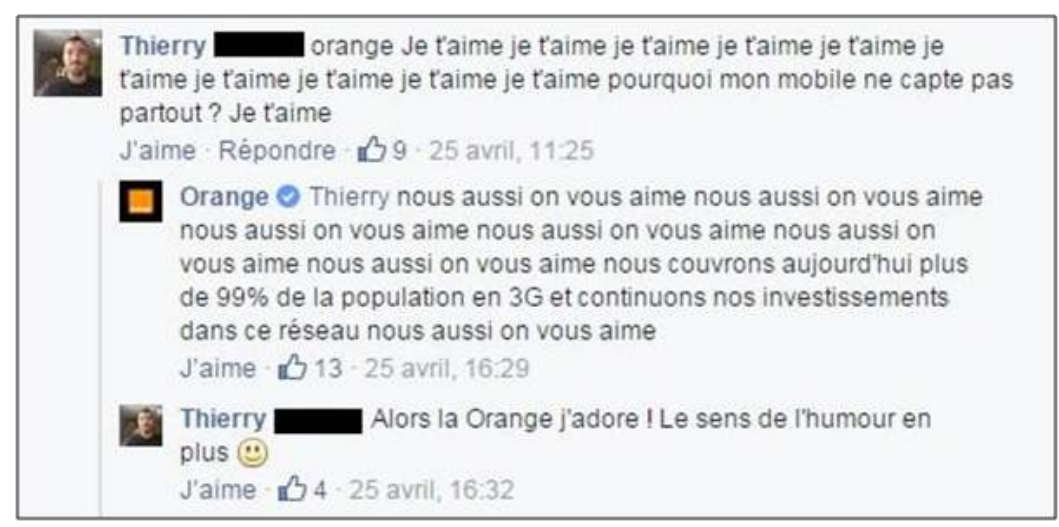

Source: Page Facebook Orange France, avril 2015.

Les webconseillers et les community managers adoptent les mêmes formes d'expression que les clients dans les interactions, celles conventionnellement utilisées sur les plateformes numériques et faisant une large place à un style relâché, à l'humour, aux onomatopées, émoticônes et autres signes véhiculant des émotions qui sont rarement exprimées dans une relation commerciale ordinaire ${ }^{17}$. Cette nouvelle convention communicationnelle à laquelle se plient les acteurs de la relation-client sur Internet, nous proposons de l'analyser au moyen de la théorie des " régimes d'engagement " développée par Laurent Thévenot dans son ouvrage L'action au pluriel ${ }^{18}$ (Thévenot, 2006).

17 Dans sa contribution, A. Mathieu-Fritz constate le même phénomène quant aux transformations qu'occasione l'usage de la télémédecine sur la relation médecinpatient. Il montre ainsi que la relation thérapeutique emprunte « dans le cadre des téléconsultations, le même type de dispositif de communication par Internet que les relations amicales, familiales, professionnelles ou commerciales [...] ; l'identité des dispositifs conduit, symboliquement, à assimiler les relations thérapeutiques par téléconsultation aux relations visiophoniques classiques et à les tenir éloignées de la relation thérapeutique traditionnelle. "

Thévenot distingue trois régimes d'engagement dans l'action. Le premier est tourné vers la contribution au bien commun et fondé sur une grandeur qualifiant pour le bien commun : il s'agit du "régime d'engagement justifiable ", développé dans De la justification (Boltanski et Thévenot, 1991). Mais en deçà de ce régime d'engagement public, il existe deux autres régimes, pour ainsi dire privés, dont le premier a vocation à assurer l'autonomie de l'individu dans la réalisation de ses intentions (c'est 
Il est évident que les community managers et les webconseillers, en tant que salariés d'entreprises fonctionnant par process, sont tributaires d'un " régime d'engagement en plan » qui implique " autonomie des acteurs ", capacité de " coordination » et mise en forme " fonctionnelle » de l'environnement. Pourtant, l'originalité de l'action de ces nouveaux intermédiaires de la relation-client est précisément de ne pas se réduire à l'exécution d'un plan, à la fourniture rapide d'informations pertinentes aux clients, à leur satisfaction. Il faut au contraire, pour que la captation soit complète, les séduire et les fidéliser, et pour ce faire sortir de l'action en plan qui ne reconnaît que des individus "autonomes " et les lie dans une forme d'engagement mutuel contractuelle (Thévenot, 2006, p. 248), pour se rapprocher des " personnes " et les reconnaître dans un engagement mutuel "attentionné " (ibid., p. 245). Nous retrouvons ainsi les constats de Julia Velkovska qui montre, dans un article qu'elle a consacré au "métier de webconseiller ", que les interactions de ceux-ci avec les clients peuvent aboutir à des formes de coopération intégrant des repères localisés (partage d'éléments de langage, identification réciproque...) permettant des rapports particularisés (Velkovska, 2015), lesquels sont tout à fait emblématiques du "régime d'engagement familier ".

\section{Conclusion}

$\mathrm{Au}$ terme de ces développements, nous pouvons revenir sur les troisième et cinquième critères retenus par Bowker pour caractériser une infrastructure informationnelle. Ces deux critères tenant respectivement à l'extension et à la transversalité des dispositifs et aux conventions et pratiques concrètes qui s'y développent nous avaient semblé mériter un examen comparatif approfondi des différentes plateformes numériques de relation-client. Cet examen permet d'établir que de telles plateformes se développent de façon transverse à trois grandes fonctions organisationnelles (l'innovation, le marketing, la vente), lesquelles sont d'ailleurs à chaque fois critiquées par les discours qui accompagnent

le " régime d'engagement en plan ") tandis que le second, plus intime encore et requérant "une attention de proximité " à la personne, vise à la mettre à son " aise ", à accommoder son environnement proche, humain et non humain (c'est le « régime d'engagement familier "). 
l'affirmation des nouveaux métiers de community manager et de webconseiller comme étant trop " cloisonnées ", trop « descendantes ", trop " mercantiles ». Ces nouveaux métiers, s'ils varient bien selon le type de plateforme considéré, ont en commun d'incarner une nouvelle posture d'interface entre l'entreprise et le client, comme un " postefrontière » de l'organisation dans le monde du Web.

Mais ce rapprochement des entreprises et de leurs publics est aussi l'occasion d'une transformation qualitative de la relation au client que l'on observe de façon transversale sur les trois types de plateformes et qui tente de concilier familiarité et captation, la première étant comme l'instrument de la seconde. Car il faut bien insister sur ce point : toutes les plateformes de relation-client entrent dans la catégorie des dispositifs de captation, et l'essor d'un régime d'engagement familier chez les animateurs de ces dispositifs ne peut être compris en dehors des contraintes marchandes de plus en plus grandes qui pèsent sur la connaissance, la conquête et la fidélisation des clients. Des travaux supplémentaires seraient requis pour déterminer si les pratiques et conventions relationnelles des community managers et des webconseillers chargés de mettre en application ce programme délicat de "captation en familiarité ", donnent lieu à une standardisation des compétences associées à ces métiers, et partant à une normalisation des fiches de poste.

Quoi qu'il en soit, il faut souligner le caractère paradoxal de cette avancée de l'entreprise vers une communication au plus proche des acteurs, une communication qui ne souhaite plus traiter seulement avec les consommateurs comme individus (avec leurs plans d'achat motivés rationnellement) mais qui les prend comme personnes (souhaitant par la consommation enrichir et modifier leur entour familier d'objets et d'usages). En effet, cette transformation peut s'interpréter de deux façons : d'un côté, elle porte au sein de l'entreprise des modes d'action et des conventions relationnelles propres à la sphère privée; de l'autre, elle participe d'un mouvement de métamorphose de cette même sphère privée, faisant davantage de place à la communication écrite, asynchrone et aux échanges marchands. Dans ce chapitre, nous avons surtout insisté sur le premier aspect de cette transformation : d'autres travaux devront s'enquérir du second. 
Finalement, sans nous avancer sur le degré d'achèvement de la dynamique de transformation organisationnelle observée, on peut bien affirmer qu'on assiste à l'essor de dispositifs et de pratiques de relation-client enchevêtrés dans un système de structures hétérogènes sur le Web, mais de plus en plus intégrés, transparents et transverses aux différentes composantes de l'entreprise. On s'achemine ainsi vers une infrastructure numérique de la relation-client s'appuyant sur des conventions relationnelles articulant familiarité dans l'échange marchand et captation du client.

\section{Références}

Benedetto-Meyer M. (2011), "Des statistiques au cœur de la relation clients : l'accès aux "données clients", leur effet sur l'organisation du travail et les relations clients/vendeurs en boutique ", Sociologies pratiques, $\mathrm{n}^{\circ} 22$, p. 49-61.

Benedetto-Meyer M., Klein N. (2015), "Entre pratique commerciale et travail réputationnel : l'invention d'une nouvelle forme relation client ? ", Terrains \& Travaux, vol. 26, $\mathrm{n}^{\circ}$ 1, p. 147-166.

Benedetto-Meyer M., Raimond E. (2011), "La relation client 2.0 : favoriser ou contraindre de nouveaux modes d'expression des clients et des salariés ? ", dans Benedetto-Meyer M. et al., L'emprise de la gestion, Paris, L'Harmattan, p. 245-273.

Benghozi P.-J. (1998), « De l'organisation scientifique du travail à l'organisation scientifique du client : l'orientation client, focalisation de nouvelles pratiques managériales ", Réseaux, vol. 16, n 91, p. 13-29.

Bergeron H., Castel P., Nouguez E. (2013), «Éléments pour une sociologie de l'entrepreneur-frontière. Genèse et diffusion d'un programme de prévention de l'obésité ", Revue française de sociologie, vol. 54, $\mathrm{n}^{\circ} 2$, p. 263-302.

Boltanski L., Thévenot L. (1991), De la justification. Les économies de la grandeur, Paris, Gallimard.

Bowker G., Noyer J.-M. (Trad.) (1997), « L'histoire des infrastructures informationnelles ", Solaris [en ligne], $\mathrm{n}^{\circ} 4$. 
Callon M. (1986), "Éléments pour une sociologie de la traduction. La domestication des coquilles Saint-Jacques et des marins-pêcheurs dans la baie de Saint-Brieuc ", L'Année sociologique, n 36, p. 170-208.

Cardon D. (2010), La démocratie Internet, Paris, Seuil, coll. « La République des idées".

Cardon D. (2011), «Sites de réseaux sociaux de l'Internet», Communications, $\mathrm{n}^{\circ} 88$, p. 141-148.

Caron F. (2010), La dynamique de l'innovation : changement technique et changement social, $X V I^{e}-X X^{e}$ siècle, Paris, Gallimard.

Carù A., Cova B. (2006), "Expériences de consommation et marketing expérientiel ", Revue française de gestion, vol. 162, n 3, p. 99-114.

Cochoy F. (2004), « La captation des publics entre dispositifs et dispositions, ou le petit chaperon rouge revisité ", dans Cochoy F. (éd.), La captation des publics. C'est pour mieux te séduire, mon client..., Toulouse, Presses universitaires du Mirail, p. 11-68.

Cochoy F. (2008), "Du lien au cœur de l'échange ", Sciences de la société, $\mathrm{n}^{\circ} 73$, p. $105-121$.

Coutant A., Stenger T. (2012), «Les sites de réseaux sociaux : une histoire de participation ", Le Temps des médias, vol. 18, n 1, p. 76-86.

Cova B., Cova V. (2002), «Tribal marketing: the tribalization of society and its impact on the conduct of marketing ", European Journal of Marketing, vol. 36, n' 5-6, p. 595-620.

Cova B., Cova V. (2009), "Les figures du nouveau consommateur : une genèse de la gouvernementalité du consommateur ", Recherche et applications en marketing, vol. 24, $\mathrm{n}^{\circ} 3$, p. 81-100.

Foray D. (2009), L'économie de la connaissance, Paris, La Découverte.

Hatchuel A., Weil B. (1995), Experts in Organization: A Knowledge-Based Perspective on Organizational Change, New York, De Gruyter.

Hatchuel A., Weil B. (1999), Pour une théorie unifiée de la conception. Axiomatiques et processus collectifs, Paris, CGS École des Mines / GIS cognition-CNRS.

Hatchuel A., Le Masson P., Weil B. (2002), « De la gestion des connaissances aux organisations orientées conception ", Revue internationale des sciences sociales, $\mathrm{n}^{\circ} 171$, p. $29-42$. 
Hetzel P. (2002), Planète conso. Marketing expérientiel et nouveaux univers de consommation, Paris, Éditions d'Organisation.

Kessous E., Mallard A., Mounier C. (2014), "À la découverte du client ", dans Kessous E., Mallard A. (éds), La Fabrique de la vente. Le travail commercial dans les télécommunications, Paris, Presses des Mines, p. 45-62.

Kessous E., Mellet K., Zouinar M. (2010), « L'économie de l'attention : entre protection des ressources cognitives et extraction de la valeur ", Sociologie du travail, $\mathrm{n}^{\circ}$ 52, p. 359-373.

Lefébure R., Venturi G. (2000), Gestion de la relation client, Paris, Eyrolles.

Licoppe C. (2002), "Le traitement des courriers électroniques dans les centres d'appels ", Sociologie du travail, vol. 44, n 3, p. 381-400.

Licoppe C. (2006), " La construction conversationnelle de l'activité commerciale. "Rebondir" au téléphone pour placer des services ", Réseaux, n 135-136, p. 125-159.

Star S. L., Ruhleder K. (1996), « Steps toward an ecology of infrastructure. Design and access for large information spaces ", Information Systems Research, vol. 7, $\mathrm{n}^{\circ}$ 1, p. 111-134.

Star S. L., Griesermer J. (1989), "Institutional Ecology, “Translations” and Boundary Objects: Amateurs and Professionals in Berkeley's Museum of Vertebrate Zoology, 1907-39 ", Social Studies of Science, vol. 19, n 3, p. 387-420.

Thévenot L. (2006), L'action au pluriel. Sociologie des régimes d'engagement, Paris, La Découverte, coll. "Textes à l'appui ».

Velkovska J. (2015), «Dans les coulisses des “communautés de marque”. Le métier du webconseiller ", Communication, vol. 33, n 2 .

Viale T. (1997), La communication d'entreprise. Pour une histoire des métiers et des écoles, Paris, L'Harmattan.

von Hippel E. (1986), "Lead Users: A source of novel product concepts ", Management Science, vol. 32, $\mathrm{n}^{\circ}$ 7, p. 791-805.

von Hippel E. (1994), "Sticky information and the locus of problem solving: implication for innovation ", Management Science, vol. ${ }^{4} 40, \mathrm{n}^{\circ} 4$, p. 429-439. 


\title{
Les infrastructures numériques comme problème professionnel
}

\author{
Denis Duplan \\ Pascal Ughetto
}

Le numérique a ses infrastructures et leur construction sociale est notamment une construction politique et professionnelle. C'est la perspective que ce chapitre se propose de développer.

Le 18 mai 2015, le Premier ministre introduit ce qu'il présente comme une seconde phase de la Nouvelle France Industrielle, un ambitieux programme annoncé le 12 septembre 2013 par le président de la République et le ministre de l'Économie, de l'Industrie et du Numérique. La Nouvelle France Industrielle y gagne des lettres de noblesse, et ses 34 "plans de reconquête pour la nouvelle France industrielle " sont refondus. Entre tous, un plan connaît une fortune particulière, celui de l'" Usine du futur ». Présenté comme source d'inspiration pour l' "Industrie du futur ", il alimente le projet phare de la seconde phase. Autour, sont " articulées " un ensemble de "solutions industrielles " qui " capitalisent » sur les autres plans.

C'est le dernier avatar d'une ambition jamais reniée, celle de développer l'industrie française, considérée en retard, puis sur le déclin, depuis la fin du « rattrapage » de l'après-guerre, qui aura successivement inspiré autant d'initiatives qu'Oséo, les pôles de compétitivité, l'Agence de l'innovation industrielle ${ }^{1}$, et la Banque publique d'investissement, pour ne citer que les plus récentes. Au regard de leur succession, il

Créée en 2005 par Jacques Chirac sur la recommandation de Jean-Louis Beffa, l'Agence de l'innovation industrielle (AII) a été supprimée par Nicolas Sarkozy. Elle était dotée de plus de deux milliards d'euros et devait notamment porter le très complexe programme Quaero, annoncé en grandes pompes par Jacques Chirac et Gérard Schröder un an plus tôt comme une riposte à Google (Duplan, 2007). 
pourrait sembler que ces initiatives, qui parfois se contredisent ${ }^{2}$, laissent sur le paysage industriel français aussi peu de traces que le vent sur la pierre. Ces initiatives ont invariablement mobilisé dans leur définition du progrès à viser une référence aux "nouvelles technologies ", dont elles ont très concrètement contribué à favoriser les usages. Au fil des années, ces technologies sont devenues les "technologies du numérique ", dont il faut rappeler que l'informatique et l'électronique en sont (encore) l'indispensable support.

Devenues omniprésentes (certains diront envahissantes), ces technologies facilitent la production et la transmission de données en permettant d'établir des infrastructures très complexes. Si de telles infrastructures ont toujours existé, leur conscience ne s'est sans doute affirmée dans l'esprit du public et/ou du décideur public, en dépit de leur caractère abscons, que depuis qu'Internet, qui en constitue une forme, est devenu pour ce public quelque chose de concret. Chacun est aujourd'hui facilement convaincu qu'il y a bien autre chose au-delà de l'ordinateur, tout un univers sous-jacent auquel il est relié et duquel il ne peut être coupé, même s'il n'en maîtrise pas tous les tenants et les aboutissants.

De fait, il est fréquent, chez les spécialistes de ces technologies, de se plaindre que les utilisateurs ne s'intéressent pas de suffisamment près à ces infrastructures. Par exemple, les jeunes générations, pourtant prétendues " nées avec ", aimeraient par-dessus tout se servir des médias sociaux, mais rechigneraient à s'intéresser à la technique sur laquelle ils reposent ; les programmes de l'école primaire devraient, quant à eux, intégrer une initiation au codage informatique dès le plus jeune âge. Bref, ces spécialistes jugent que l'utilisateur ordinaire se complaît trop facilement dans son identité de profane et ne se rend pas suffisamment conscient des besoins de création et de maintenance de ces infrastructures. La figure sociale du " geek » ne valide-t-elle pas l'existence d'une partition entre des utilisateurs investis, connaissant le fonctionnement technique

L'AII de Jean-Louis Beffa soutenue par Jacques Chirac s'inscrivait clairement en opposition avec les pôles de compétitivité de Christian Blanc soutenus par Nicolas Sarkozy, l'idée étant de renforcer les grands industriels plutôt que d'assister les startup (ibid.). 
et acteurs de ce fonctionnement, et une plus grande masse d'utilisateurs, surtout désireux que les infrastructures opèrent, de manière fluide, sans rupture, avec un débit rapide et fiable?

Les frontières entre amateurs et professionnels (Flichy, 2010) en ressortent à la fois révisées et consolidées. Autour du fonctionnement de l'infrastructure, des amateurs s'investissent comme de quasiprofessionnels et, en plus grand nombre, des utilisateurs utilisent chaque jour les outils numériques et ont recours aux infrastructures correspondantes tout en considérant leur fonctionnement comme un mystère épais ou, plus vraisemblablement, comme une donnée dont ils ne se soucient pas. L'important, pour eux, c'est que cela fonctionne, sans avoir à se préoccuper de la façon dont c'est le cas.

Dans tout cela, l'activité sous-jacente au développement et à l'entretien des infrastructures numériques demeure peu décrite et interrogée. Ce chapitre cherchera à montrer que celles-ci n'existent en partie que dans la mesure où du travail leur est consacré, un travail qui n'est pas simplement un travail de réalisation et de maintenance des équipements (Denis, Mongili et Pontille, 2015), mais aussi d'élaboration des doctrines de ce que doivent être ces infrastructures et les professions légitimes pour les prendre en charge. Tout technicien que ce travail puisse être, il est indissociablement un travail normatif. C'est aussi l'enjeu d'un débat. Des professions ou des segments professionnels (Strauss, 1992) débattent de ce qui est bien et de ce que sont les pratiques légitimes au bénéfice de quelles infrastructures numériques. Les trajectoires effectives d'infrastructures numériques doivent beaucoup à des formes de division du travail, répartissant les tâches et traduisant la façon dont ces segments professionnels envisagent le travail à effectuer pour les concevoir et les entretenir.

Pour cette raison, le chapitre proposera d'ouvrir la porte des directions de systèmes d'information, les DSI, que l'on trouve désormais obligatoirement dans toutes les grandes entreprises. C'est là que l'on verra le numérique s’imposer, non comme une substance déjà constituée, mais en actes, par l'intermédiaire de la construction de mots d'ordre, de pratiques professionnelles ordinaires, de normes de ce qui a de la valeur pour l'entreprise. Ces DSI ne sont pas des mondes sociaux homogènes, mais sont, en particulier, traversées par la cohabitation, toute en rivalité, des développeurs de logiciels et des gestionnaires de l'infrastructure 
matérielle - les devs et les ops, dans le vocabulaire indigène. Le territoire professionnel des uns et celui des autres ne sont pas donnés de façon stable et l'essor du "cloud » n'est pas loin de constituer une menace pour les seconds. Mais tout ne se joue pas au seul niveau des entreprises, ces enjeux professionnels étant en partie arbitrés par l'État et les politiques publiques.

\section{Ce que le numérique fait à l'informatique}

Le numérique est, au premier abord, le triomphe de l'informatique, mais se prétend autant son dépassement. À partir de 2013, sous l'impulsion du syndicat professionnel Syntec informatique, qui s'était déjà rebaptisé Syntec numérique plusieurs années auparavant, les sociétés de services en ingénierie informatique (SSII) se rebaptisent entreprises de services numériques $(\mathrm{ESN})^{3}$. Il s'agit clairement d'épouser une époque où le "numérique "s'est imposé comme un enjeu spécifique pour le décideur public auquel s'adresse avant tout un syndicat professionnel pour défendre sa vision des intérêts de ses adhérents.

De fait, le "numérique » est désormais inscrit à l’agenda public. Un secrétariat d'État notamment chargé du « développement de l'économie numérique " est apparu pour la première fois sous la présidence de Nicolas Sarkozy en 2009, détenu alors par Éric Besson, et les gouvernements successifs ont depuis toujours comporté un portefeuille qui le mentionne - depuis 2014, il n'est d'ailleurs plus question d' "économie numérique ", mais de "numérique " tout court, même si le rattachement à l'économie perdure. Tout cela est même en passe d'être supplanté par le " digital ", depuis que les dirigeants font, quel que soit leur secteur d'activité, le déplacement au Consumer Electronics Show de Las Vegas.

Même parmi les principaux intéressés par sa promotion, la notion de «numérique » a visiblement pris un certain temps à étendre sa portée au-delà de celles de l'" informatique ". Les dix propositions adressées par le Syntec numérique aux candidats à l'élection présidentielle de 2012 ne diffèrent pas grandement en nature de celles qu'il défend

Voir https://syntec-numerique.fr/ssii-esn/esn-ssii (consulté le 18 juillet 2017). 
depuis longtemps ${ }^{4}$ (Syntec numérique, 2012). Il s'agit toujours d'obtenir des aides de l'État, sous la forme d'allègement de charges, d'aménagement du droit du travail, de création de marchés publics, de prise en charge de la formation, toutes choses qui peuvent faciliter le recours à la main-d'œuvre et l'accès à des marchés où employer celle-ci. Les " infrastructures numériques " ne sont mentionnées qu'une fois, prises dans une acceptation la plus évidente, celle du très haut débit, non sans parenté avec les autoroutes de l'information de l'ère ClintonGore. Somme toute, le "numérique " peut alors encore passer pour un synonyme $\mathrm{d}^{\prime}$ " informatique ».

La situation a certainement évolué depuis. Mi-septembre 2014, le secrétaire d'État chargé de la Réforme de l'État et de la Simplification présente un projet de recours au numérique pour transformer l'État. Au-delà des mesures de simplification, il s'agit d'engager une "véritable stratégie numérique " qui doit conduire l'État à investir dans ses infrastructures, désignées comme "le système d'information de l'État " ${ }^{5}$. Ce n'est certainement pas la seule initiative récente des décideurs politiques en matière de "numérique " - nous avons évoqué notamment l'«Industrie du futur » en introduction -, mais celle-ci témoigne d'une particulière appropriation du "numérique » par l'État, puisqu'il prétend se l'appliquer.

Notons que cette appropriation du "numérique " témoigne d'un débordement vis-à-vis de l' "informatique ». À la limite, cette dernière n'apparaît plus que comme un moyen pour faire du "numérique ». On rejoint ici la question évoquée plus tôt d'une partition entre l'univers des spécialistes qui maîtrisent la technique sur laquelle l'outil repose et éventuellement l'outil -, et celui des profanes qui ne maîtrisent que l'outil. Sous l'influx de la généralisation du propos sur le «numérique», et tout particulièrement de l'action publique qui cherche à le promouvoir, cette partition qui renvoyait autrefois à celle des spécialistes et des profanes en « informatique » évolue vers une partition entre spécialistes de l'« informatique » et spécialistes du "numérique ».

4 Disponibles à l'adresse : https://syntec-numerique.fr/actu-informatique/10-propositions-syntec-numerique-candidats-election-presidentielle-2012 (consulté le 18 juillet 2017).

5 Compte rendu du Conseil des ministres du mercredi 17 septembre 2014. 
L’« informatique » ne disparaît certes pas avec le «numérique ", mais elle rejoint les coulisses. Les infrastructures évoquées par le secrétaire d'État sont bien techniques, puisqu'il évoque le système d'information. Toutefois, c'est de "numérique " dont il est question avant tout. L'appropriation de la notion par les pouvoirs publics contribue à la politiser, car dans le champ de l'action politicienne, il devient toujours plus difficile d'ignorer ce sur quoi une autre partie s'appuie pour fonder son action. Le "numérique " devient un sujet d'affrontement quand il entre dans son champ autre chose que du consensuel, par exemple la question très sensible du droit d'accès à l'information, comme l'ont illustré les débats sur le projet de loi pour une République numérique présenté fin 2015.

Tout ne se réduit pas, pour autant, à des stratégies sémantiques pour ennoblir le champ. À mesure que le Web s'est développé et a pris l'ascendant sur les enjeux et les usages de l'informatique, il a provoqué des déplacements, des déformations, voire des mutations. Ainsi, la traditionnelle opposition entre l'infrastructure (le hardware) et les logiciels (le software) semble remise en cause par l'essor du cloud et des logiciels en mode software as a service (SaaS). Au sein de ce mode, les entreprises n'achètent plus les licences des logiciels et n'implantent plus ceux-ci sur les équipements de leurs salariés, mais accèdent à distance à leurs données en louant un droit d'usage de l'application. Les entreprises y trouvent leur compte en s'épargnant la maintenance des logiciels. Mais, comme a pu le souligner Yannick Fondeur à propos des applications de ce modèle dans le domaine de la gestion du recrutement, "le mode SaaS déplace le curseur en faveur du standard " (Fondeur et Lhermitte, 2013, p. 12) : les entreprises clientes font nécessairement usage du même logiciel, les personnalisations sont réduites au minimum, de l'ordre du paramétrage. "Cette opération consiste à choisir des éléments (fonctionnalités, règles de gestion, processus...) parmi une gamme de possibilités définies a priori sans remise en cause du modèle de données du logiciel. Ainsi, les modalités d'adaptation du standard se limitent à celles prévues en amont par le dispositif technique : pour être utilisable, une fonctionnalité doit préalablement avoir été inscrite dans le standard. Le paramétrage se résume ainsi à l'activation/désactivation d'options " (ibid., p. 13). 
Dans son chapitre, dans cet ouvrage, Antoine Picon évoque une première couche des infrastructures, le substrat technique. Évidemment, dans l'informatique, qu'il y ait du hard et du soft ne signifie pas que le substrat technique ne soit que du premier côté. Hard et soft sont deux modalités du substrat technique. Ou encore, celui-ci est inextricablement constitué de ces deux modes d'existence. Néanmoins, les discours qui vantent l'entrée dans une nouvelle ère, numérique ou digitale, le font en suggérant que comptent désormais avant tout les données - notamment quand elles sont massives, les big data. Si le cloud est un support décisif de collecte massive de données, le cas très précis du SaaS suggère que les infrastructures numériques soulèvent des problèmes qui ne s'y limitent pas. Les infrastructures logicielles à distance entraînent volontiers de forts effets de standardisation et, notamment, engendrent des effets de standardisation des infrastructures de gestion telles qu'évoquées dans le chapitre de Gilles Jeannot et Pascal Ughetto (dans cet ouvrage). En termes théoriques, c'est, dans le vocabulaire d'A. Picon, la couche des régularités et des usages qui est ici concernée et cela conforte très sensiblement l'intérêt des sujets sur lesquels les infrastructure studies attirent notre attention : celui des standards, à la fois comme codage de l'information et comme normes de comportement.

Si l'on pénètre dans les organisations que sont les entreprises ou les administrations clientes de ces logiciels, on s'apercevra que la montée du numérique n'est absolument pas neutre pour certains acteurs en particulier : Y. Fondeur (2014) l'a montré à propos de la fonction ressources humaines, dont les pratiques de recrutement se trouvent structurées par des acteurs extérieurs à l'entreprise et à leur champ professionnel. C'est, on l'imagine, tout autant le cas pour les directions des systèmes d'informations et leurs membres. Le déplacement des infrastructures vers le cloud équivaut à des formes de dépossession et ne peut qu'avoir des répercussions sur les pratiques professionnelles. Mais, tout autant, et pour emprunter à nouveau à A. Picon, c'est l'imaginaire qui préside à ces pratiques professionnelles qui est interrogé.

$\mathrm{Au}$ cas du cloud pourraient être ajoutés d'autres exemples. Mentionnons simplement ce que certains en sont venus à dénommer, de façon légèrement marketing, le bring your own device (BYOD, pour les initiés). La capacité des salariés à se connecter grâce à leur smartphone à des applications qui leur sont usuelles dans leur vie personnelle équivaut 
à une mise en concurrence des outils que les DSI voulaient prescrire de manière exclusive. Une perte de contrôle se manifeste ici. Â différents niveaux, par conséquent, les infrastructures numériques sont des enjeux d'acteurs et des problèmes professionnels.

\section{Ce que le numérique fait aux DSI}

\section{La lutte des DSI pour faire reconnaître leur valeur}

Avant que la connectivité au domicile ne connaisse une subite accélération avec la démocratisation de l'accès à Internet et que le concept d'infrastructure ne se popularise dans la foulée, ce concept était déjà largement répandu dans l'entreprise, tout particulièrement la grande entreprise.

D'un point de vue formel, telle qu'elle se donne à voir au visiteur qui en franchit le seuil, une grande entreprise est invariablement organisée par directions, classées entre directions métier (ceux qui font) et directions support (ceux qui permettent de faire), l'ensemble étant coiffé par la Direction générale. Cette dernière est au-dessus des parties, dans les deux sens du terme. En effet, le visiteur comprend rapidement que chaque direction poursuit ses objectifs propres à l'intérieur de ceux de l'entreprise, et qu'elle peut à cette occasion entrer en conflit avec une autre direction dont les objectifs ne recoupent pas les siens. Si un tel conflit s'envenime, c'est la Direction générale qui, située au-dessus, tranche.

Dans ces conditions, avoir l'oreille de la Direction générale constitue toujours pour une direction un enjeu, qui se traduit très concrètement par la possibilité de siéger dans des instances que cette Direction organise pour élaborer ses décisions (Flamant, 2002). Il n’est jamais bon de passer pour une direction support, étant considérée comme l'intendance qui suivra, qu'on pourrait à ce titre écarter de l'élaboration des décisions, et comme un centre de coûts et non de profits, que l'on pourrait à ce titre toujours plus enjoindre à se restreindre.

À cet égard, la position de la Direction des systèmes d'information (DSI), direction qui a spécifiquement en charge ce qui ressortit au matériel et au logiciel informatique, paraît incertaine. La DSI, direction support ou direction métier? 
Force est de constater que la DSI est au fil du temps passée de la tour d'ivoire à la citadelle assiégée. Dans les années 1980, l'économiste Robert Solow énonçait son fameux paradoxe : "You can see the computer age everywhere but in the productivity statistics ${ }^{6}$. "Plus récemment, l'essayiste Nicholas Carr créait la polémique en publiant dans la Harvard Business Review "IT doesn't matter " (Carr, 2003)7. Contre de telles imprécations, en France, le combat de la DSI pour avoir son mot à dire sur la stratégie de l'entreprise est notamment porté par le Club informatique des grandes entreprises françaises (Cigref).

Sur ce point, on peut se reporter à une étude exemplaire conduite avec McKinsey, conseil de la Direction générale s'il en est (Cigref et McKinsey, 2008). La première conclusion est que pour gagner voix au chapitre, " être irréprochable dans l'exécution est en effet essentiel pour asseoir la légitimité de la DSI ». Voilà qui peut s'avérer décevant pour une direction qui souhaitait certainement être reconnue comme stratégique de droit, de ne pouvoir espérer l'être qu'en fonction de sa capacité à servir les autres. Toutefois, ce n'est pas tout. En effet, démontrer sa capacité à servir constituerait une injonction à laquelle la DSI pourrait facilement se plier si elle ne devait pas craindre d'être mise sur la touche par le recours à des acteurs concurrents. Confrontées à ce qu'elles estiment être une inertie de la DSI là où il ne s'agit bien souvent pour cette dernière que de répondre aux besoins de manière organisée, les directions peuvent développer leur informatique interne clandestine - shadow IT - en s'appuyant sur leurs propres ressources, voire en mobilisant des ressources externes (ZDNet.com, 2012). Ces comportements décrédibilisent doublement la DSI, non seulement parce qu'ils reviennent à la court-circuiter, mais aussi parce qu'ils lui compliquent sa tâche de fond, à savoir contenir l'entropie du système d'information (SI).

Le « numérique » constitue ici une nouvelle menace pour la DSI. Dans plusieurs entreprises où l'un des deux auteurs (D. Duplan) est intervenu en 2015, le "virage numérique " ou la «transformation numérique " était à l'ordre du jour. Affiché comme un nouvel eldorado, le "numérique »

$\overline{6}$ «On peut voir l'ère de l'informatique partout, sauf dans les statistiques de productivité" (nous traduisons).

7 Prenant acte des évolutions technologiques une dizaine d'années après, l'auteur a expliqué comment son point de vue a évolué dans une interview à Network World. 
constituait l'objet des attentions sinon de la Direction générale, du moins de la direction marketing, générant une pression sur la DSI pour s'emparer du sujet. Soulignons ce point : il est des organisations dans lesquelles le numérique est un projet autant porté par des directions du marketing ou de la communication que par les DSI. À partir de là, diverses configurations ont pu être constatées, de la refonte radicale de la DSI sur la base d'un projet de tout " numérique " à la création d'un laboratoire de l'innovation, ou « lab ", éventuellement sous contrôle de la DSI, procédant à du prototypage rapide. Ces observations font écho au discours relayé dans la presse professionnelle, appelant une énième fois le Directeur des systèmes d'information (DSI) à se transformer au risque d'être détrôné, cette fois, par un "Directeur du numérique " sorti du bois (ZDNet.com, 2014). On parle désormais de " chief digital officer " (CDO).

\section{Domestiquer le numérique en tant que DSI}

Qu'y a-t-il de nouveau dans le "numérique " pour la DSI ? De prime abord, il paraît singulier que la direction la plus en phase avec l'informatique soit menacée d'un débordement par un mouvement qui aurait trait à l'informatique. Il faudrait, pour cela, définir le " numérique ", mais pas plus que le "Web 2.0 ", la notion ne se prête facilement à l'exercice.

À lire l'étude McKinsey citée par ZDNet.com, ce serait «big data and advanced analytics, digital engagement of customers, digital engagement of employees and external partners, automation, and digital innovation " (McKinsey, 2013). On sent confusément qu'il est question d'une capacité d'ouvrir massivement le SI à des utilisateurs sur lesquels la DSI ne peut pas, ou ne peut plus, exercer le même contrôle qu'avant, et de le faire en s'inscrivant dans des usages innovants, tout particulièrement en valorisant les données collectées auprès de ces nouveaux utilisateurs.

Quoiqu'a priori moins surplombant, un document tel qu'Entreprise 2020 à l'ère du numérique produit par le Cigref ne permet pas de mieux cerner le sujet (Cigref, 2014) ${ }^{8}$. Il est question de relever une série de

8 Le Cigref semble avoir réitéré, avec sa réflexion sur la transformation numérique, l'effort qu'il avait déployé des années plus tôt pour réfléchir sur la création de valeur par le SI. 
" défis " qui s'imposeraient à l'entreprise du fait de phénomènes aussi variés que l'abaissement des barrières à l'entrée sur les marchés, la multiplication des " espaces de création de valeur ", la perte de monopole de l'entreprise sur l'innovation, le développement du cloud et du big data, etc., tout cela relevant de grandes tendances que sont la mondialisation, la "servicisation » et l'apparition de technologies de rupture. De là des préconisations tous azimuts, de "Réinventer le modèle d'affaires... ou disparaittre ? " à " Développer la culture numérique des femmes et des hommes » en passant par « Repenser l'organisation pour mieux innover $"$.

S'il ressort une certitude de tout cela pour la DSI, c'est la nécessité qui s'impose à elle de réagir au discours sur le "numérique " pour ne pas donner l'impression d'être dépassée par ce dernier. De ce fait, l'enjeu apparaît plus peser sur les épaules du DSI que sur celles de sa DSI. Au-delà de sa personne, il est possible que ce soit pour ses équipes business as usual : contenir l'entropie du SI tout en étendant ce dernier en répondant à de nouveaux besoins avec de nouvelles technologies.

\section{Ce que le numérique fait aux informaticiens}

\section{Devs et Ops : hiérarchies symboliques internes au monde des informaticiens autour de l'« infra »}

Dans le contexte d'une DSI, parler d'" infra " pour dire " infrastructure ", c'est évoquer un pan bien spécifique de la DSI qui regroupe les personnes s'occupant de la vie du matériel qui permet de faire fonctionner les logiciels, les $O p s$. Ce pan se distingue donc de celui qui regroupe les personnes s'occupant de la conception et de la production desdits logiciels, les Devs.

Devs et $O p$ s sont classiquement opposés, jusqu’à la caricature. Dans un épisode de la récente série Silicon Valley, l'Op Gilfoyle installe un ensemble de serveurs dans le garage de la start-up et repousse ironiquement la proposition du Dev Chugtai de s'en mêler ${ }^{9}$ : "You

Pour Gilfoyle, l'infrastructure est bien évidemment au-dessus de tout soupçon, la mauvaise performance ne pouvant tenir qu'au logiciel : "My servers can handle 10 times the traffic if they weren't busy apologizing for your shit codebase. " "Mes 
don't do this kind of work, remember? You make a living with your mind, not your hands. [...] Why don't you go inside and write some princess code? Leave the hardware up to us servants ${ }^{10}$."

D'ailleurs, quand Chugtai y mettra les mains malgré tout, il provoquera une coupure de courant générale, faute d'avoir compris ce qu'il faisait. C'est qu'à chacun son métier et, au-delà, des objectifs considérés antagonistes. Si les deux parties ont bien en charge le système d'information (SI), ce n'est pas de la même manière. Ainsi, l'exemple d'une DSI d'une banque où nous avons pu intervenir. D'un côté, les Devs doivent répondre à des demandes des autres directions, parfois sous l'effet de nouvelles exigences réglementaires (ex. : la mise en œuvre des nouvelles normes de prélèvement SEPA). Il leur faut pouvoir mettre en service des logiciels ou modifier des logiciels déjà mis en service, ce qui peut impliquer une transformation de l'infrastructure qui permet à ces logiciels de fonctionner. Leur mission première est donc d'assurer l'évolution du SI. De leur côté, les $O p s$ doivent répondre aux demandes des Devs en mettant à disposition des infrastructures pour permettre aux logiciels de fonctionner. Toutefois, devant assurer les premiers niveaux de support aux utilisateurs, ils doivent avant tout veiller à ce que les logiciels qui fonctionnent bien continuent de bien fonctionner. Leur mission première est donc d'assurer la stabilité du SI.

\section{DevOps : les infrastructures et la coopération en mode agile?}

Concilier ces visions divergentes fait l'objet d'un courant de pensée nommé DevOps (Programmez!, 2014). L'idée est de faciliter le travailler ensemble en renforçant la prise en compte des contraintes de l'autre. Le schéma honni est celui où les Devs ne sollicitent les $O p s$ qu'au terme du développement d'un logiciel, quand il s'agit de déployer ce dernier précipitamment pour tenir des délais sans avoir permis aux $O p s$ de l'anticiper, les infrastructures étant assimilées à une intendance qui devra

serveurs pourraient servir 10 fois le trafic s'ils n'étaient pas contraints de s'excuser pour ton code de merde.") (nous traduisons).

10 "C'est pas ton genre de boulot, tu te souviens? Tu vis de ton cerveau, pas de tes mains. [...] Pourquoi pas rentrer et écrire du code de princesse ? Laisse le matériel à nous, les gueux » (nous traduisons). 
suivre. À ce schéma, on souhaite substituer celui où les Devs échangent tôt et régulièrement avec les $O p s$, et s'appuient sur des outils pour faciliter le déploiement et des métriques pour en superviser la fiabilité, ce qui revient à « transformer le SI en self-service pour les développeurs, gérés par les $O p s »(i b i d$.

L'exigence d'une telle collaboration s'impose dans un contexte où les Devs fonctionnent toujours plus souvent sur un modèle " agile " (cf. encadré). Pour les Devs, cela se traduit par la nécessité de procéder à des déploiements plus fréquents, et donc pour les $O p s$, à d'autant plus de sollicitations.

\section{L'agilité en informatique... et au-delà}

Le terme fait référence à un principe dont se sont revendiqués, dans un Manifeste agile élaboré en 2001, un certain nombre de Devs auteurs de méthodes de conduite de projets d'un type particulier à partir de la fin des années 1990 (Agile, 2001).

Ces méthodes tranchent sur de nombreux points sur la manière dont la production d'un logiciel a initialement été approchée, c'est-à-dire en déroulant un processus de production inspiré du BTP, très segmenté et coupé du client - le modèle dit " de la cascade " : spécification, production, test, livraison.

L'objectif d'une méthode agile est de limiter la déception que crée l'écart entre les besoins exprimés et ceux auxquels le logiciel répond finalement (ou prétend répondre sans y parvenir car, n'ayant pas été assez testé, il dysfonctionne). Pour cela, la méthode prescrit de nombreuses pratiques, tout particulièrement le fait d'itérer des cycles de production brefs au terme desquels la demande est ajustée à l'aune des progrès accomplis dans la production de la réponse qu'on y apporte. Au-delà, il s'agit aussi de responsabiliser chacun en faisant en sorte qu'il s'attribue lui-même son travail, de prendre au sérieux l'estimation des délais requis par ceux qui développent, etc.

C'est l'adoption grandissante des méthodes agiles par les équipes en charge de projets informatiques qui est à l'origine de l'émergence du concept d'" agilité ", désormais érigé en principe de réorganisation de l'entreprise que le manager moderne se doit de mettre en ouvre. La fortune de la réappropriation s'opère indéniablement au prix d'une reformulation radicale, tout devant être " agile " désormais dans l'entreprise, jusqu'au contrat de travail, dans un contexte français où les employeurs apprécient toujours de trouver un substitut diplomatique au terme de flexibilité.

Il est clair qu'une DSI, et au sein d'une DSI des groupes de Devs et d'Ops bien dissociés, ne se rencontrent que dans certaines entreprises. Pour autant, il serait bien difficile de dégager les critères 
qui déterminent une telle structuration. Quelque part, il existe toujours dans une entreprise une personne responsable de l'informatique, et cette personne ainsi que celles qui éventuellement en dépendent ont toujours une prédilection pour tels ou tels aspects de l'informatique qui contribueraient à les classer comme Devs ou comme $O p s$.

\section{Les Ops sous la menace du cloud et d'une externalisation des infrastructures?}

Pour les $O p s$, il est intéressant de relever que l'émergence d'infrastructures numériques, pour le coup entendues dans le sens précis des plateformes matérielles et logicielles partagées auxquelles il est possible d'accéder via Internet et qui sont maintenues par un tiers - c'est-à-dire le cloud $^{11}$-, constitue sinon un problème, du moins un enjeu professionnel.

En mars 2012, Adrian Cockcroft, directeur de l'architecture des systèmes cloud de Netflix, une célèbre plateforme de diffusion de contenus vidéo sur Internet, a largement contribué à faire enfler une polémique initiée quelques mois plus tôt en parlant de "NoOps " à Netflix (Proffitt, 2012). NoOps, au sens strict auquel Cockcroft l'entend, renvoie à une organisation du travail où les Devs déploient eux-mêmes leurs logiciels sur des plateformes dans le cloud, s'appuyant sur des outils plutôt que sur des $O p s$.

Si NoOps ne semble pas avoir fait fortune, la polémique a eu l'intérêt de souligner combien les $O p s$ pouvaient se trouver sous la menace d'une externalisation des infrastructures qui ne remonte pas à hier, mais qui est certainement devenue plus pressante avec le développement de services permettant à qui n'est pas $O p$ de jouer lui-même de nombreux paramètres pour lesquels l'intervention d'un $O p$ était auparavant requise ${ }^{12}$.

11 On distingue dans le cloud plusieurs niveaux de services proposés, dont essentiellement : le matériel constituant l'infrastructure au sens premier du terme (Iaas, pour Infrastructure as a Service), l'infrastructure et le système d'exploitation (Paas, pour Platform as a Service), le logiciel fonctionnant sur ces bases (SaaS, pour Software as a Service).

12 On parle un peu de CloudOp sur Internet : présenté comme le futur de l'Op dans un article (http://www.evolven.com/blog/cloudops-the-reinvented-it-ops.html), mais le terme ne semble pas avoir fait fortune (du moins, pour l'heure). Dans Silicon 


\section{Conclusion}

À la base, des innovations technologiques suscitent de nouveaux usages et en remettent en cause d'anciens, ce qui incite à dresser le constat de l'entrée dans une nouvelle ère. Un terme est forgé pour caractériser le mouvement, c'est le "Web 2.0 " ou la "transformation numérique ", qui ne cesse d'être investi de sens par tous ceux qui y trouvent l'opportunité de faire valoir leurs intérêts en les présentant sous ce jour. Avec le temps, il n'est pas dit que ces termes ne perdent pas du lustre que leur confère leur nouveauté (ils sont d'ailleurs aujourd'hui volontiers supplantés par le " digital »), tandis que la mémoire de ce à quoi ils renvoient se fait plus incertaine. Le résidu de l'attracteur prend le sens caractérisant au mieux le mouvement que l'attracteur a engendré. Tout comme " 2.0 » est devenu aujourd'hui synonyme d'aggiornamento sous toutes ses formes, "numérique " semble bien en passe de devenir synonyme de connectivité tous azimuts. D'une certaine manière, la connectivité, c'est l'homme désormais relié aux infrastructures avec lesquelles il échange des données, au point que les frontières de l'homme sont remises en cause comme jamais. S'il fallait, parvenus à ce stade, retenir comme définition que le numérique, c'est la connectivité, alors l'infrastructure numérique serait ce qui permet cette connectivité sous toutes ses formes.

Au fond, quoi de neuf avec la transformation numérique ? L'informatique n'a-t-elle pas toujours été le lieu d'un progrès rapide, comme la loi de Moore ${ }^{13}$ à elle seule pourrait en témoigner ? N'est-elle pas non plus à ce titre un cimetière d'innovations dont le souvenir s'est perdu, même s'il en est bien resté quelque chose, comme la distance qui nous sépare de Napster ou de Second Life pourrait le rappeler ? Par ailleurs, le progrès de l'informatisation n'est-il pas marqué par un mouvement de contraction et de dilatation des infrastructures informatiques qui ne cessent à chaque itération de s'étendre : du centre

Valley, l'Op Gilfoyle prétend qu'aucune infrastructure dans le cloud ne peut rivaliser avec celle qu'il a bâtie de toutes pièces dans le garage... Et l'équipe doit recourir aux compétences d'un petit génie du cloud quand il est question d'y venir, parce que déployer sur le cloud n'est pas dans ses cordes.

13 Gordon E. Moore, alors directeur de la R\&D Fairchild Semiconductor et qui deviendra l'un des trois fondateurs d'Intel, est resté célèbre pour avoir prédit en 1965 que le nombre de transistors dans un circuit intégré doublerait chaque année. 
de calcul en temps partagé au mini auquel des terminaux sont connectés, au personal computer qui embarque la puissance du mini, au serveur si puissant qu'on ravive un temps l'idée du terminal, à l'Internet où tout objet comporte un ordinateur?

Il faut traiter ces modes comme il conviendrait de le faire pour les modes managériales. Tout d'abord, ce n'est pas parce que ce sont d'abord des paroles ou des actes de langage que ce n'est que du vent. Le numérique recouvre ou débouche bien sur des problèmes très réels à surmonter, par exemple pour les $O p s$. Au minimum, le problème est de ne pas perdre leur place, aussi bien leur emploi (dans l'hypothèse où l'imagination débordante de certains parviendrait à inventer réellement des solutions permettant de se passer des Ops dans les DSI) que leur position symbolique, déjà sans cesse contestée par des Devs volontiers portés à les faire passer pour des banals intervenants techniques. Mais le problème est aussi, face aux injonctions prononcées en Codir ou Comex - les stratégiques comités de direction ou comités exécutifs - de procéder à des réalisations faisant une place à ces réalités (cloud ou autre) dont, initialement, on ne pensait pas grand bien ou que l'on regardait avec un peu de distance. Le problème, c'est encore de devoir combattre pour s'assurer suffisamment de considération de la part d'autrui (collègues de la DSI, direction générale...) pour conserver une possibilité de travailler selon ses normes.

\section{Références}

Agile (2001), Manifeste pour le développement logiciel agile.

Carr N. (2003), "IT doesn't matter ", Harvard Business Review, May.

Cigref et McKinsey (2008), Dynamique de création de valeur par les Systèmes d'information: une responsabilité partagée au sein des Directions des Grandes Entreprises, juillet.

Cigref (2014), Entreprise 2020 à l’ère du numérique. Enjeux et défis, octobre.

Denis J., Mongili A. et Pontille D. (éds) (2015), « Maintenance and repair in STS ", Tecnoscienza, vol. 6, n² 2, p. 5-111. 
Duplan D. (2007), «Quaero, un objet encombrant. Analyse sociologique d'une initiative visant à mobiliser l'industrie et la recherche publique française dans le contexte européen ", Mémoire Master 2 de l'action : organisation, marché, régulation politique, dir. Pierre Veltz, Sciences Po Paris.

Flamant N. (2002), Une anthropologie des managers, Paris, PUF.

Flichy P. (2010), Le sacre de l'amateur, Paris, Le Seuil.

Fondeur Y. (2014), "La "professionnalisation du recrutement" au prisme des dispositifs de sélection ", Revue française de socio-économie, vol. 14, $\mathrm{n}^{\circ} 2$, p. $135-153$

Fondeur Y. et Lhermitte F. (2013), "Outils informatiques de gestion de recrutement et standardisation des façons de recruter ", Document de travail du CEE, $\mathrm{n}^{\circ} 165$, avril.

McKinsey (2013), Bullish on digital: McKinsey Global Survey results, août.

Proffitt B. (2012), "NoOps: Divining the Future of the Network », Linux Magazine, $\mathrm{n}^{\circ} 140$, juillet.

Programmez! (2014), "DevOps : développement \& production ", $\mathrm{n}^{\circ}$ 180, décembre, p. 37-41.

Strauss A. (1992), La trame de la négociation, Paris, L'Harmattan.

ZDNet.com (2012), "Cachez-moi cette informatique que je ne saurais voir ", 15 décembre.

ZDNet.com (2014), "La nouvelle mission numérique du DSI », 14 mai. 

Plateformes et infrastructures virtuelles 



\title{
Les plateformes, étude d'une infrastructure numérique
}

\author{
Patrice Flichy
}

Dans l'histoire des réseaux, Internet est un cas tout à fait spécifique, puisque le "réseau des réseaux " s'est créé comme une " noninfrastructure ». Pour l'essentiel, Internet a adopté la stratégie du coucou, il a utilisé les infrastructures téléphoniques et celle d'un réseau de données universitaires pour se développer et croître extrêmement rapidement. Plus exactement, Internet est une infrastructure virtuelle qui a défini des formats de données, des règles de transmission, des logiciels de navigation, des moteurs de recherche... Dans la perspective des pères fondateurs, c'est un espace d'échange qui fait disparaître les intermédiaires ${ }^{1}$. Sur la toile, chaque internaute peut entrer en contact avec n'importe quel autre, pour lui fournir de l'information, lui proposer un service... Mais à la désintermédiation initiale, succède une phase de ré-intermédiation. De nouveaux intermédiaires vont prendre la place de ceux qui ont été évincés. Ce ne sont pas, à proprement parler, des commerçants en ligne, ils fournissent simplement la connexion entre offreurs et demandeurs. À l'origine, on trouve des sites non marchands de petites annonces comme Craiglist fondé en 1995. Puis, au-delà de l'accès aisé à l'information sur les différentes offres et sur les prix associés, de nouveaux sites vont permettre de réduire les coûts de coordination et de recherche de partenaires. Pour l'internaute, ils vont jouer un rôle essentiel, ils facilitent le contournement des gatekeepers du commerce. Comme sur la place de marché traditionnel, chacun peut venir proposer ces produits à des utilisateurs qui peuvent être proches ou distants. La tradition démocratique d'Internet s'est étendue aux mondes des échanges aussi bien marchands que non marchands.

Sur les débuts d'Internet (Abbate, 1999 ; Flichy, 2001), sur la nouvelle économie (Malone et Laubacher, 1998). 
Car les plateformes n'organisent pas seulement les échanges matériels, mais aussi les immatériels. Dans l'utopie initiale d'Internet, chacun peut accéder à l'information et plus largement aux contenus qu'il veut, et publier ce qu'il souhaite en court-circuitant les autorités légitimes, les prescripteurs, les spécialistes attitrés, chacun peut produire de l'information, noter, évaluer et même recommander des biens culturels. Ce projet qui est devenu l'idéologie commune d'Internet est en phase avec cette volonté des individus de s'autonomiser.

Dans la tradition des travaux du LATTS sur les infrastructures techniques, je me propose d'étudier ces plateformes, qui sont souvent analysées en termes d'économie collaborative, d'économie du partage ou d'économie pair-à-pair, comme une infrastructure virtuelle. J'étudierai, tout particulièrement, le mode d'articulation entre l'offre et la demande, l'organisation des bases de données et les algorithmes (Flichy, 2013). Ces éléments infrastructurels sont à la base de cette économie des plateformes susceptible de modifier profondément le travail et la répartition de la plus-value (Kenney et Zysman, 2016).

Je ne me propose pas ici de présenter une théorie générale des plateformes comme peuvent le faire les économistes ${ }^{2}$, mais au contraire de décrire la diversité de ces dispositifs en étant attentif à leur genèse et à leur évolution. Je prendrai l'exemple d'une dizaine de plateformes dans le domaine des médias (YouTube), des petites annonces (Leboncoin), dans le champ de la rédaction d'avis sur les restaurants (Tripadvisor), de la vente d'objets d'artisanat (Etsy), du covoiturage (Blablacar), du transport des personnes (Uber), de la livraison par coursier (Deliveroo), de l'hébergement de tourisme (Couchsurfing, Airbnb), du repas chez l'habitant (VizEat), de la réparation à domicile (Youpijob, Lulu dans ma rue) et du crowdsourcing (Amazon Mechanical Turk, Upwork).

Le phénomène des plateformes est devenu tellement foisonnant qu'il est nécessaire d'en distinguer deux types : les plateformes de mise en visibilité de l'information ou de la production culturelle qui constituent en quelque sorte une nouvelle forme médiatique et les plateformes de mise en relation d'une offre et d'une demande de biens ou de services.

En économie, les plateformes constituent un exemple paradigmatique de la théorie des marchés bifaces. Voir Rochet et Tirole (2003). Voir également Evans et Schmalensee (2017). 
Après avoir présenté ces deux types de plateformes, j’examinerai plus spécifiquement le rôle de leurs algorithmes, comment elles se situent à la frontière du marché et du hors-marché, du travail permanent et du travail d'appoint, du local et du mondial et enfin comme elles remettent en cause la réglementation des professions existantes.

\section{Les nouveaux médias}

Internet a profondément modifié le système médiatique en donnant la possibilité à chaque internaute de s'exprimer. Là où l'information était contrôlée par les journalistes et les éditeurs, le réseau digital a fait sauter les gatekeepers (Cardon, 2010) et a donné la possibilité à tous de prendre la parole sur un blog, puis sur les réseaux sociaux. Les amateurs de nombreuses disciplines culturelles peuvent mettre leurs performances à la disposition de tous. YouTube permet de réaliser le vieux rêve de la radio au début du $\mathrm{XX}^{\mathrm{e}}$ siècle, puis de la télévision par câble trois quarts de siècle plus tard, permettre à chacun de diffuser une émission sonore ou une vidéo (pour une analyse de ces expériences au milieu des années 1970, voir Barbier Bouvet et al., 1979). Chaque minute, plus de 300 heures de vidéo sont mises en ligne. Plus d'un milliard d'utilisateurs uniques consultent YouTube chaque mois. La plateforme qui est un immense succès se finance par la publicité, comme la plupart des médias.

Les plateformes entrent également en concurrence avec d'autres activités supportées par les médias, la critique et les petites annonces. Comme dans le domaine de l'information, les jugements et avis sur les produits culturels (musique, cinéma) ou sur les hôtels et les restaurants ne sont plus rédigés exclusivement par des experts : critiques de musique ou de films ou rédacteurs de guide, mais par les internautes eux-mêmes qui notent, jugent, évaluent. L'évaluation ne concerne plus, comme auparavant, une partie de l'offre dite de qualité, mais l'intégralité : tous les films ou les livres qui sortent, les petits restaurants... Par ailleurs, l'évaluation est ouverte à tous. Elle est moins sujette à caution que les avis des experts qu'on peut toujours soupçonner d'être manipulés. Le premier slogan de la plateforme d'évaluation de restaurants Tripadvisor, Reviews you can trust, illustre bien cette défiance face aux jugements des experts. La participation est multiple : critiques argumentées, 
évaluations rapides, partage d'une émotion, dénonciation d'un service qui ne correspond pas à ce qui était attendu. Face à cette diversité d'avis, la plateforme met en place des dispositifs d'agrégation, elle produit une note de synthèse. Ce calcul réalisé par un algorithme est une nouveauté qui distingue fortement la plateforme d'avis, des rubriques critiques des médias. Si l'idée initiale de ces sites est de dénoncer la critique des experts et de promouvoir une critique faite par tous, in fine, on s'aperçoit que grâce à la magie de l'algorithme ces sites deviennent eux-mêmes de nouveaux leaders d'opinion.

Les petites annonces constituent également un service issu de la presse. Ce n'est d'ailleurs pas un hasard si Leboncoin.fr a été lancé par deux groupes de presse Ouest France et le groupe norvégien Schibsted. Comme dans le cas précédent, la plateforme modifie profondément le service précédent. L'informatique permet de trier les annonces et facilite le " matching " entre l'offre et la demande, ainsi les petites annonces peuvent être affichées dans un dispositif national, tout en conservant leur dimension locale. C'est ainsi que Leboncoin est devenu l'acteur dominant du secteur.

Les plateformes qui s'intègrent dans le monde des médias se sont d'abord présentées, selon la tradition des pères fondateurs d'Internet, comme une alternative à un système centralisé, contrôlé par les gatekeepers. Elles voulaient héberger une production amateur en pleine croissance, faciliter la vente et l'achat par des particuliers des biens multiples. Mais petit à petit, ces acteurs alternatifs sont devenus des acteurs dominants capables de concurrencer la presse et la télévision grâce à la puissance de leur dispositif d'hébergement, capables aussi de structurer les échanges de biens entre particuliers.

\section{Les plateformes de transaction}

L'autre famille de plateformes organise la rencontre de l'offre et la demande. Elles sont nées pour démocratiser les échanges, permettre à ceux qui réalisent pendant leur temps libre des objets faits à la main, de les vendre (Etsy), faciliter la rentabilisation de son capital domestique, en louant sa voiture ou son logement (Drivy, Airbnb), exploiter ses

$\overline{3}$ Ouest France s’est retiré par la suite. 
compétences ordinaires (enfourcher un vélo, conduire une voiture, faire la cuisine) en transportant des passagers, en effectuant des livraisons instantanées, ou en préparant un repas pour des visiteurs de passage (Uber, Deliveroo, VizEat). En examinant comment se sont organisées ces plateformes, les règles de l'échange, les principes de l'algorithme, le cadrage des offreurs et des demandeurs, nous verrons comment on est passé d'un site sympathique qui facilitait l'échange à un intermédiaire influent qui structure un secteur économique.

Prenons d'abord l'exemple d'Etsy. La plateforme permet à des productrices de bijoux, d'objets de décoration ou d'art graphique de proposer leur production à des clients, en court-circuitant les boutiques physiques qui prennent une marge très élevée (autour de $40 \%$ ) et en accédant à un large marché. Etsy prend une marge faible (7,5\%), assure la sécurisation du paiement et ouvre sur le marché mondial. Ainsi, de nombreuses vendeuses écoulent une forte part, voire la majorité de leur production à l'étranger. La plupart des productrices ne consacrent qu'une petite partie de leur temps à cette activité, seule une petite minorité se procure l'essentiel de ses revenus sur la plateforme.

Le modèle d'Etsy est celui d'une plateforme qui est là pour faciliter la vente, c'est une galerie virtuelle de boutiques en ligne. Lespace numérique permet à la plateforme d'accueillir sans limites les propositions des fabricantes d'objets faits main. À ce modèle qui s'appuie sur une offre déjà constituée, on peut opposer le modèle inverse qui est celui où il y a une demande spécifique qui cherche un offreur pour y répondre. Les champs couverts sont très divers. Ces plateformes de crowdsourcing peuvent proposer des tâches très peu qualifiées comme Amazon Mechanical Turk ou des taches plus créatives comme Upwork, ou du bricolage comme Youpijob ou Lulu dans ma rue. Amazon Mechanical Turk demande à ceux qu'on appelle souvent les travailleurs du clic d'effectuer des travaux très courts et répétitifs, comme transcrire les achats d'un panel de consommateurs qui envoient une photo de leur ticket de caisse, décrypter un enregistrement sonore, décrire succinctement une photo ou tout simplement répondre à une enquête. Sur Upwork, le travail est beaucoup plus qualifié, il s'agit de concevoir un site web, de réaliser des illustrations, de faire des traductions ou d'écrire du code. 
À côté de ces plateformes qui cherchent à faire correspondre à une offre ou à une demande déterminée une clientèle ou un fournisseur, on trouve enfin des plateformes dont le cœur de l'activité est d'imaginer un nouveau service. C'est dans le domaine du déplacement (Uber, Blablacar) ou de l'hébergement (Couchsurfing, Airbnb) que ces plateformes se sont d'abord développées, elles s'appuient sur des ressources (voitures, hébergements) proposées par les ménages. La plateforme doit assurer l'appariement d'offre et de demande qui sont localisées et situées dans le temps.

Quel que soit le cas de la figure, la caractéristique des plateformes est de se présenter comme un intermédiaire. Contrairement à un commerçant classique, elles n'achètent pas un bien pour le revendre, elles n'embauchent pas des professionnels qui vont fournir un service qu'elles commercialisent, elles n'ont pas à investir dans un parc de voitures ou d'hébergements. Le cœur de l'activité de la plateforme est de constituer deux bases de données, celle des offreurs, celle des demandeurs et d'organiser l'appariement à travers un algorithme. Les plateformes sont donc bien les infrastructures du commerce en ligne. Elles n'ont pas les équipements et la main-d'œuvre du commerce, mais elles fournissent les dispositifs qui rendent possible un commerce en ligne. Comme souvent les infrastructures, elles ne se contentent pas de rendre possible une activité, mais elles la formatent, de plus en plus l'intermédiaire devient l'acteur principal.

Le formatage est d'abord imposé par la nécessité de structurer la base de données. L'organise-t-on autour des acteurs, offreurs ou demandeurs, ou autour des biens ou services proposés ? Etsy prend comme premier critère de classement de l'offre, la figure de l'artisan. Son tour de main est ce qui le distingue de ses concurrents. Etsy est donc une galerie virtuelle de boutiques d'artisans. Upwork organise, au contraire, la demande des prestations à fournir, selon les caractéristiques du service à fournir et non en fonction de l'identité du demandeur. Sur Uber, le service est complètement homogénéisé, puisque le client ne choisit pas une voiture et un chauffeur spécifique.

L'autre élément de cadrage du service est la fixation du prix, qui peut être laissée à l'offreur (Etsy) ou au demandeur (Upwork), selon celui qui initie la relation commerciale, ou au contraire est orientée par la plateforme (BlablaCar propose, ainsi, un mode de calcul de prix 
du trajet - essence et péage - divisé par trois passagers et conseille de rester dans une modulation de plus ou moins $50 \%$ ) ou assurée par la plateforme, comme le font Uber ou Deliveroo qui imposent leurs tarifs.

\section{L'algorithme}

Lalgorithme est évidemment l'élément infrastructurel clé de la plateforme, puisque c'est lui qui propose et hiérarchise les appariements. Dans la plupart des plateformes, il fait des propositions et l'internaute peut ensuite regarder les notes qui ont été attribuées, lire les commentaires. Pour les activités de transport à la personne ou de livraison, la plateforme fait directement une proposition d'appariement qui peut éventuellement être refusée. L'algorithme fait son classement en fonction des notes données par les internautes, mais aussi des commentaires et du nombre de like. Pour les objets faits main, s'y ajoute un paramètre de nouveauté, pour l'hébergement ou le transport à la personne, la disponibilité de l'offre et la proximité géographique de l'offreur et du demandeur. Airbnb propose à l'usager des logements disponibles dans la ville ou le quartier choisi et Uber connecte les clients et les chauffeurs qui sont les plus proches.

Mais l'algorithme est une boîte noire, les plateformes ne dévoilent que quelques-uns des paramètres du calcul. D'autres variables ne sont pas indiquées. Etsy tient compte du nombre de ventes, de l'ancienneté, du nombre de produits que présente la boutique. Airbnb privilégie les pages qui sont mises à jour le plus régulièrement, c'est-à-dire celles des loueurs professionnels au détriment des loueurs particuliers, qui constituent l'image de marque du site. De même, Uber, dans une même zone de proximité géographique, hiérarchise les voitures proposées non seulement en fonction de la note des chauffeurs, mais surtout en fonction du modèle et de l'âge de la voiture. Ainsi, en formatant les bases de données et l'algorithme, les plateformes ne sont plus seulement ces intermédiaires qui permettent de développer le travail ouvert (Flichy, 2017), ce travail mené à l'extérieur de l'entreprise, par des travailleurs indépendants, elles deviennent de nouvelles entreprises de service innovantes qui offrent d'autres services : paiement, assurances, etc. 
Cette nouvelle économie des plateformes s'inscrit dans la filiation de l'Internet, elle est donc face à plusieurs dilemmes que le réseau des réseaux a eu à résoudre : l'activité est-elle marchande ou non marchande? S'agit-il d'un travail réalisé par des travailleurs à plein temps ou au contraire d'une activité amateur menée à côté dans la filiation des hackers ? Ces services sont-ils locaux comme les premiers services électroniques ou mondiaux comme le Net? Comment se positionnentils face aux services existants et aux professions en place?

\section{Gratuité ou paiement}

L'économie des plateformes est souvent appelée économie collaborative. Cette appellation insiste sur le fait qu'on est dans une économie où les individus cherchent à optimiser les ressources, en matière d'hébergement, de transport, mais aussi d'utilisation d'objets de seconde main. Ces ressources réemployées ne s'intègrent pas toujours dans l'économie marchande, elles relèvent aussi du don. C'est dans le domaine des services de logement et de mobilité que la frontière entre le don et le paiement est la plus ténue. On peut recevoir ou transporter quelqu'un gratuitement, en le faisant participer aux frais ou en le faisant payer. Il s'agit de service voisin, sachant néanmoins que le don s'inscrit dans la création d'un lien, ce qui n'est pas le cas du marché (Hyde, 2007 [1983]). Mais accueillir des dons et des ventes est finalement assez proche : le rôle essentiel de la plateforme, quelle que soit sa nature, est de fonctionner de façon rapide et efficace, de cadrer l'échange et de veiller à ce qu'à la fin les deux interlocuteurs soient quittes.

Dans l'autre type de plateformes, celles qui relèvent des médias, la gratuité est généralement la norme. Aussi, on accuse souvent (Cardon et Cassili, 2015) ces plateformes d'exploiter le travail gratuit. Pour l'essentiel des Youtubeurs, cette accusation n'a pas grand sens. Il s'agit de productions amateurs qui souhaitent simplement trouver un public qui dépasse celui des amis et des proches. Pour eux, la question de la rémunération ne se pose pas. La situation est différente pour les professionnels. Certes, un très petit nombre de nouveaux entrants obtiennent des revenus de leurs vidéos. Ces vidéos regroupées dans des chaînes doivent capter une audience importante pour dégager des revenus effectifs (un million de visionnements procurent un revenu 
d'environ 1000 euros). Seuls quelques types de vidéo, comme les sketches humoristiques, peuvent atteindre une telle audience (Beuscart, 2015). Aussi les chaînes plus spécialisées, comme les chaînes consacrées à l'histoire, adoptent un ton humoristique et malgré cela ne dépassent pas 400000 visionnements (Miller, 2016). Quant aux professionnels déjà en place, comme les chanteurs, ils se plaignent de recevoir de la plateforme une rémunération beaucoup plus faible que celle qu'ils reçoivent des éditeurs de $\mathrm{CD}$.

\section{Travail partiel ou permanent}

Le lien des plateformes avec le monde de l'Internet des origines fait qu'au démarrage, les offreurs, comme les amateurs ou les blogueurs, menaient cette activité en parallèle, ils prêtaient une chambre sur Couchsurfing, puis ensuite, ils l'ont loué sur Airbnb, ils faisaient $\mathrm{du}$ covoiturage ou transportaient des personnes dans leur voiture, moyennant finance. Cela reste majoritairement le cas aujourd'hui. Une enquête réalisée aux États-Unis en 2016 monte que $24 \%$ des adultes américains ont gagné de l'argent sur une plateforme en ligne durant l'année écoulée : vente en ligne, $18 \%$, dont $14 \%$ d'objets de seconde main ; services en ligne, $8 \%$; hébergement, $1 \%$ (Smith, 2016). En France, on estime que 18,5 millions de personnes ont acheté ou vendu un objet sur Leboncoin, pour un revenu annuel moyen d'environ 400 euros, hors vente immobilière et de voiture (Prudhomme, 2017).

Même Uber, qui est devenu l'exemple emblématique de l'économie des plateformes, utilise principalement des chauffeurs qui exercent cette activité à côté de leur métier principal. C'est notamment le cas aux ÉtatsUnis, où toute personne ayant une voiture et un permis de conduire peut transporter des passagers. Aussi, outre-Atlantique, les deux tiers des chauffeurs Uber ont une autre activité et $55 \%$ conduisent moins de quinze heures par semaine (Hallet et Krueger, 2016). En France, où l'activité équivalente intitulée UberPop a été interdite, suite à la pression des chauffeurs de taxi, Uber fait appel à des chauffeurs qui ont une licence de VTC, c'est dans $70 \%$ des cas leur activité principale. La moitié d'entre eux travaillaient préalablement dans le secteur des transports. La 
plateforme offre aussi une possibilité d'emploi aux chômeurs ( $25 \%$ des chauffeurs étaient au chômage avant de démarrer cette activité, dont $43 \%$ au chômage depuis plus d'un an) (Landier et al., 2016).

On semble voir une évolution voisine chez les livreurs à vélo de Deliveroo ou de Foodora. Si, à l'origine, il s'agissait le plus souvent d'étudiants passionnés de vélo, aujourd'hui, on trouve plus de livreurs dont c'est l'activité principale. Là aussi, ils sont largement issus des banlieues populaires (Dablanc, 2017). Sur la plateforme Amazon Mechanical Turk, qui fonctionne au niveau mondial, seul le quart des travailleurs du clic travaillent à plein temps (Hitlin, 2016).

\section{Local/Mondial}

L'activité des plateformes a souvent une forte dimension locale, acheter un bien de seconde main à petit prix, se faire conduire à la gare, faire faire une réparation chez soi... Et pourtant, les plateformes sont souvent organisées à une échelle géographique plus large. Le site du Boncoin est national. Parmi les plateformes de VTC, des sociétés françaises comme Marcel ou Le cab ont démarré à Paris et s'étendent dans quelques villes de province, mais Uber lui s'est lancé au niveau mondial. Dans les petits travaux à domicile, Youpijob a démarré en France et s'est développé dans d'autres pays européens, par contre Lulu dans ma rue a mis en place une organisation géographique par quartier parisien. Ce choix lui permet d'avoir des modes de communication multiples avec les demandeurs. La plateforme reçoit les demandes par téléphone ou dans un espace physique, un kiosque de quartier et choisit les prestataires (les Lulu) de façon semi-automatique, les recherches sur les tâches faciles à effectuer étant, en effet, dirigées vers des prestataires peu expérimentés. L'évaluation par les clients se fait la première fois par téléphone et ensuite par SMS. Ce dispositif qui associe le contact humain à l'informatique est une exception.

Quand les plateformes sont complètement informatisées, la tendance est, au contraire, de proposer les services de la plateforme à une échelle la plus large possible au niveau national, européen, comme Blablacar, ou mondial comme Uber. Un tel choix est justifié par le fait que les plateformes ont des coûts fixes informatiques et promotionnels importants, elles jouent donc au maximum sur le facteur taille. 


\section{Réglementation}

Les plateformes remettent en cause les professions existantes. Pour pouvoir développer leurs activités, elles sont souvent amenées à contourner les réglementations existantes. Les plateformes de livraison instantanée sont un bon exemple de ces stratégies. La réglementation française impose à tout livreur utilisant un véhicule à deux roues motorisé de s'inscrire au registre des transporteurs légers. Pour pouvoir bénéficier d'une large population de livreurs directement mobilisables, les plateformes, comme Deliveroo, ont fait appel à des cyclistes qui eux n'ont pas cette contrainte (Dablanc, 2017).

Ce travail de contournement de la réglementation est particulièrement visible dans le transport des personnes puisque les plateformes de VTC remettent en cause le numerus clausus des taxis. Dans le domaine de l'hébergement, c'est l'homologation qui est remise en cause. Alors qu'un gîte doit avoir un label, avec différents niveaux de classement, tout hôte peut proposer une chambre sur Airbnb. Dans le domaine de l'hôtellerie et de la restauration, les règles de sécurité et d'hygiène sont particulièrement importantes. Les hôtes qui offrent des logements ou des prestations de social dining, comme VizEat, peuvent s'en affranchir puisqu'ils reçoivent chez eux et non dans des lieux publics. Le site VizEat encadre cette pratique en faisant signer aux hôtes une charte dans laquelle ils s'engagent à « respecter la réglementation relative à l'hygiène et à la salubrité du lieu, des aliments et des boissons proposés ". Le site a également prévu une assurance pour couvrir les hôtes, comme les invités. Ainsi, les plateformes remplacent le règlement par le contrat et l'assurance. Elles sont donc des acteurs de la déréglementation, d'autant plus puissants qu'elles sont souvent mondialisées et peuvent s'appuyer sur les cadres juridiques les plus ouverts, pour les diffuser dans les pays aux réglementations plus rigides.

\section{Conclusion}

À l'origine, les plateformes n'étaient que des intermédiaires discrets permettant aux internautes de mettre leurs productions en ligne, de proposer des services, finalement de pratiquer le travail ouvert. Petit à petit, elles sont devenues des infrastructures puissantes qui cadrent ce 
nouveau secteur d'activités. L'économie des plateformes s'est d'abord développée dans le non marchand, puis s'est étendue au secteur marchand, sans perdre, pour autant, son rôle d'organisation du travail gratuit. Bien que des dispositifs locaux continuent à fonctionner, les plateformes se structurent de plus en plus au niveau mondial. Elles posent donc des questions non résolues en termes de politiques publiques. Elles contournent la loi et les États ont bien du mal à les réguler. Enfin, les plateformes ne sont pas des entreprises standards qui font des investissements matériels et embauchent pour rendre un service. Elles se contentent de manager des offreurs de service. Est-ce le signe d'une mutation majeure du capitalisme ? Dans ce cas, nous entrerions dans la fin du salariat, le travail serait, maintenant, effectué par des indépendants. Cette thèse se heurte, néanmoins, à l'un des constats que nous avons faits, précédemment, la plupart des offreurs de biens ou de services sur les plateformes n'exercent pas cette activité à plein temps, ils font cela " en plus ». Les données statistiques françaises sur l'emploi indiquent d'ailleurs que si le nombre d'emplois non salariés a augmenté de 2008 à 2013, de 9,1 \% à 10,4\%, cela vient essentiellement des auto-entrepreneurs qui pour une bonne partie d'entre eux mènent cette activité conjointement avec un autre travail ou pendant leur chômage (INSEE, 2016). Le salariat reste le statut de référence du marché de l'emploi. Si les plateformes ouvrent donc de nouvelles frontières à d'autres formes de travail, elles ne constituent pas pour autant la forme dominante du travail de demain.

\section{Références}

Abbate J. (1999), Inventing the Internet, Cambridge (Mass.), The MIT Press.

Barbier Bouvet J.-F., Beaud P., Flichy P. (1979), Communication et pouvoir : mass media et media communautaires au Québec, Paris, Anthropos.

Beuscart J.-S. (2015), « La conversion de la notoriété en ligne. Une étude des trajectoires de vidéastes pro-am ", Terrains \& travaux, n 26, p. 83-104.

Cardon D. (2010), La démocratie Internet. Promesses et limites, Paris, Seuil, coll. « La république des idées ». 
Cardon D., Cassili A. (2015), Qu'est-ce que le digital labor ?, Bry-surMarne, INA.

Dablanc L. (2017) «Le boom des livraisons instantanées ", TEC Mobilité intelligente, $\mathrm{n}^{\circ} 233$, avril.

Evans D., Schmalensee R. (2017), De précieux intermédiaires. Comment Blablacar, Facebook, Paypal ou Uber créent de la valeur, Paris, Odile Jacob.

Flichy P. (2001), L'imaginaire d'Internet, Paris, La Découverte.

Flichy P. (2013), « Rendre visible l'information. Une analyse sociotechnique du traitement de données ", Réseaux, vol. 31, n 178-179, p. 55-90.

Flichy P. (2017), Les nouvelles frontières du travail à l'ère numérique, Paris, Seuil.

Hallet J., Krueger A; (2016), «An Analysis of the Labor Market for Uber's Driver-Partners in the United States ", National Bureau of Economic Research Working Papers, $\mathrm{n}^{\circ} 22843$, novembre.

Hitlin P. (2016), « Research in the Crowdsourcing Age, a Case Study: How Scholars, Companies and Workers Are Using Mechanical Turk, a "Gig Economy” Platform, for Tasks Computers Can't Handle », PewInternet. org, 11 juillet.

Hyde L. (2007 [1983]), The Gift. Creativity and the Artist in the Modern World, New York, Vintage Books.

INSEE (2016), «Enquête emploi. Le revenu moyen d'un auto-entrepreneur est de $410 €$ », INSEE Première, $\mathrm{n}^{\circ} 1627$, décembre.

Kenney M., Zysman J. (2016), "The Rise of the Platform economy ", Issues in Science and Technology, Spring, p. 61-69.

Landier A., Szomoru D., Thesmar D. (2016), «Travailler sur une plateforme Internet. Une analyse des chauffeurs utilisant Uber en France », 4 mars, https://drive.google.com/file/d/0B1s08BdVqCgrTEZieTloQnRlazQ/ view (consulté le 27 juin 2017).

Malone T., Laubacher R. (1998), "The Dawn of the e-lance Economy ", Harvard Business Review, September-October, p. 147-148.

Miller M. (2016), « Professeurs, étudiants, amateurs... Ils refont l'histoire sur YouTube ", Le Monde Campus, 16 décembre.

Prudhomme C. (2017), «Comment Leboncoin s'est imposé dans notre vie quotidienne ", Le Monde, 25 mars. 
Rochet J.-C., Tirole J. (2003), «Platform Competition in Two-Sided Markets ", Journal of the European Economic Association, vol. 1, n 4.

Smith A. (2016), Gig Work: Online Selling and Home Sharing, PewInternet. org, 17 novembre. 


\title{
La gestion de crise : une infrastructure à part entière ?
}

\author{
Valérie November \\ Servane Gueben-Venière
}

Ce chapitre propose une lecture exploratoire de la gestion de crise. Plutôt que de considérer cette activité comme ad hoc, se révélant uniquement lors d'événements graves, nous proposons de la considérer comme une infrastructure en tant que telle. Pour ce faire, nous devrons élargir la conception habituelle de la gestion de crise, qui est pensée surtout comme une action à mener afin de faire en sorte que la crise reste sous contrôle.

L'action consistant à gérer, réguler une crise, est notamment permise grâce à l'existence d'infrastructures techniques et numériques (câbles électriques, connexions électroniques, ordinateurs, réseaux routiers pour n'en citer que quelques-unes). Toutefois, si la gestion de crise ne semble être activée - et avec elle les infrastructures techniques et numériques qui l'appuient - uniquement lorsqu'une crise surgit, son existence va au-delà de l'événement déclencheur de la crise. La préparation des acteurs, en amont de l'événement déclencheur de crise, de même que la gestion des conséquences à moyen et long termes d'une crise, témoignent d'une pérennité de la gestion de crise qui dépasse le seul moment de son activation visible. C'est pourquoi, en nous appuyant sur la littérature de la sociologie des sciences et techniques (STS), nous proposons de repenser la gestion de crise en l'analysant comme une infrastructure, au sens large du terme. Il ne s'agit donc pas tant d'examiner un processus d'infrastructuralisation de la gestion de crise, qui consisterait à dire que la gestion de crise subit une transformation requérant un nombre croissant d'infrastructures, mais plutôt de porter un nouveau regard sur la gestion de crise en la considérant comme une infrastructure à part entière, d'ores et déjà existante. Cet angle d'approche nous permet d'une part de mieux comprendre comment le temps constitue une 
caractéristique centrale de cette infrastructure globale qui se différencie ainsi d'autres formes d'infrastructures, et d'autre part, d'examiner le passage délicat de l'invisibilité à la visibilité. En d'autres termes, envisager la gestion de crise comme une infrastructure à part entière nous permet d'aller au-delà de la durée d'une situation de crise et de porter aussi notre regard sur ce qui reste en sommeil en dehors d'une crise, sur ce qui n'est pas rendu explicitement visible, mais qui, pourtant, structure et conditionne l'existence même de la gestion de crise.

La réflexion que nous proposons dans ce chapitre s'appuie sur un travail de terrain réalisé dans le cadre du programme de recherche EURIDICE ${ }^{1}$ monté en partenariat avec le Secrétariat Général de la Zone de Défense et de Sécurité de Paris (SGZDS), en charge de la gestion de tous types de crises survenant en Île-de-France. L'année 2015-2016 a consisté à observer le travail effectué en cellules de crise, pendant des crises réelles de différente ampleur (attaques terroristes de novembre 2015, crue de la Seine en juin 2016), des exercices de gestion de crise (EU Sequana, exercice européen de gestion d'une crise provoquée par une crue centennale de la Seine) ou encore le suivi de grands événements (COP21 et EURO 2016). Seule l'étude de deux exemples a été retenue ici : les attaques terroristes caractéristiques d'une crise à cinétique rapide et la crue de la Seine, caractéristique d'une crise à cinétique lente. Au total ces deux exemples ont permis de cumuler plus de 200 heures d'observation de la gestion de crise mise en œuvre à l'échelle zonale. Ces observations in situ ont été complétées par la conduite d'une trentaine d'entretiens semi-directifs, auprès des personnels de gestion de crise présents en cellules de crise pendant nos observations.

La première partie de ce papier fait un tour de la littérature et s'arrête sur une définition de l'infrastructure telle qu' elle a été développée en STS. La deuxième partie nourrit cette proposition conceptuelle des exemples tirés de nos observations en cellules de crises et des entretiens réalisés. Enfin, la troisième partie insistera sur les zones d'ombre, peu visibles ou invisibles pendant une crise et pourtant incontournables à prendre en compte pour comprendre la gestion de crise dans son entièreté et comme relevant d'un assemblage modulable, faisant infrastructure.

Équipe de recherche sur les risques, dispositifs de gestion de crise et événements majeurs, programme débuté en septembre 2015, coordonné par Valérie November. 


\section{Gestion de crise et infrastructure : une relation à repenser}

\section{De l'infrastructure critique pour appréhender la gestion des crises...}

De façon générale, le thème de la gestion des crises croise la littérature sur les infrastructures dans un domaine particulier, celui des infrastructures critiques. Cette littérature s'est essentiellement développée à partir des années 2000, suite à l'occurrence de plusieurs grands événements ayant marqué la mémoire collective : le World Trade Center à New York en 2001 (Mendonça et Wallace, 2006), l'ouragan Katrina à la Nouvelle Orléans en 2005 (Boin et McConnell, 2007), le tsunami en Indonésie en 2004, les attaques terroristes à Madrid en 2004 et Londres en 2005 (Boin et Smith, 2006). La tendance s'est renforcée suite aux événements plus récents, survenus dès 2010, notamment le triple accident au Japon en 2011, les attaques terroristes à Paris en 2015. Non pas que le thème soit ignoré avant - le terme apparaît vers le milieu des années 1990 dans les documents officiels (Galland, 2010) -, mais la discussion va porter de façon de plus en plus inquiète sur la vulnérabilité croissante des infrastructures, due à l'augmentation de leurs interconnexions (LaPorte, 2007). Ainsi, le recours aux infrastructures critiques pour caractériser la gestion des crises met l'accent sur les conséquences en chaîne initiées par une défaillance de l'infrastructure critique elle-même, pouvant mener à des crises de grande ampleur, qu'il s'agisse de crises à cinétique rapide telles des attaques terroristes (Boin et Smith, 2006) ou de crises à cinétique lente telle une crue majeure de la Seine. À cet égard, les recherches d'Arjen Boin et Allan McConnell sont les plus pertinentes, puisque ceux-ci examinent les ruptures dans le domaine des infrastructures critiques à partir de la littérature sur les crises et les catastrophes (Boin et McConnell, 2007).

Toutefois, si la notion de rupture du système permet de relier infrastructures critiques et crises (OCDE, 2003), il faut noter que la notion d'infrastructure critique elle-même a des contours flous (Galland, 2010) et qu'elle varie de pays en pays. L'Union européenne définit les infrastructures critiques comme "les installations physiques et les technologies de l'information, les réseaux, les services et les actifs, 
qui, en cas d'arrêt ou de destruction, peuvent avoir de graves incidences sur la santé, la sécurité »(Rostum et al., 2008). Cette définition est reprise par plusieurs pays tels l'Allemagne, le Royaume-Uni, la Suisse, les Pays-Bas, les États-Unis ou encore la Norvège (Galland, 2010). Toutefois, les États-Unis par exemple élargissent leur définition de l'infrastructure critique en y incluant les services d'urgence et de secours (Boin et McConnell, 2007). De son côté, la Norvège va même plus loin en considérant qu'une infrastructure critique :

" ... relève des actifs et des systèmes qui sont essentiels pour maintenir les fonctions critiques de la société, c'est-à-dire ses besoins de première nécessité ainsi qu'un sentiment de sécurité pour la population. L'infrastructure critique comprend les infrastructures suivantes : électricité, télécommunications, eau potable et eaux usées, transport, oléoducs et réseaux de gaz. Les fonctions critiques de la société comprennent la circulation fiduciaire, l'alimentation, les services sociaux et de santé, la police, les services de sécurité-sauvetage, la gestion de crise, le gouvernement, la cour de justice, la défense, le contrôle environnemental et la gestion des déchets " (Rostum et al., 2008).

Ainsi, à la lumière de ces définitions, le lien que l'on peut établir entre gestion de crise et infrastructures critiques semble pouvoir dépasser la seule notion de rupture et de défaillances en chaîne pouvant conduire à des crises. Ce constat se lit également lorsque, symétriquement, on considère les outils et structures de coordination qui permettent la gestion de crise tels une unité de coordination ou un groupe interorganisationnel ou encore un réseau informel d'intervenants, comme le souligne M.-C. Therrien (2010). La gestion de crise s'appuie sur d'autres infrastructures préexistantes, d'ordre technique certes, mais aussi matériel, organisationnel et décisionnel. En ce sens, et comme dans d'autres domaines tels la télémédecine (A. Mathieu-Fritz dans cet ouvrage) ou les plateformes numériques (P. Flichy dans cet ouvrage), elle ne se suffit pas à elle-même. Elle est au contraire en totale interdépendance avec les réseaux, câbles et autres services. C'est le cas, pour ne citer qu'un exemple, des cellules de crise, spécifiquement construites et imaginées pour permettre la gestion de crise et qui ne sauraient exister sans câbles électriques, connexions électroniques, ordinateurs et téléphones permettant une solide coordination entre les cellules de crise activées par des acteurs tant publics que privés. 
Pour dépasser ce lien entre infrastructure critique et gestion de crise, établi par la notion de rupture, nous proposons de nous concentrer désormais plus précisément sur une définition plus large de l'infrastructure et de voir s'il est possible et sous quelles conditions d'envisager la gestion de crise comme une infrastructure à part entière.

\section{... à la gestion de crise faisant infrastructure}

Les travaux de Susan Leigh Star, Geoffrey Bowker et Karen Ruhleder proposent de définir une infrastructure par 8 dimensions, ou plus précisément de considérer l'agencement de ces dimensions pour former une infrastructure sans frontières ni définition a priori (Star et Bowker, 2006) :

1) encastrement : l'infrastructure est contenue, comme si elle était «coulée » à l'intérieur d'autres structures, arrangements sociaux et technologies ;

2) transparence : l'infrastructure est transparente pour l'usager, c'est-à-dire qu'il n’y a besoin ni de la réinventer à chaque fois, ni de l'assembler pour chaque tâche, tout en étant un soutien invisible de ces tâches ;

3) portée ou étendue : elle peut être spatiale ou temporelle ; l'infrastructure va au-delà d'un événement isolé ou d'une pratique unique ;

4) apprentissage comme bénéfice de l'appartenance : prendre pour acquis des artefacts et des arrangements organisationnels est une condition sine qua non pour faire partie d'une communauté de pratiques (Lave et Wenger, 1999; Star, 1991). Les étrangers et ceux qui viennent de l'extérieur rencontrent l'infrastructure comme un objet qu'il faut apprendre à utiliser ;

6) incorporation des normes et des standards : l'infrastructure, modifiée par la portée et souvent par des conventions contradictoires, devient transparente en puisant dans d'autres infrastructures et outils de manière standardisée ;

7) construire sur une base installée : l'infrastructure ne vient pas de rien ; elle lutte contre l'inertie de la base installée et hérite des forces et limites de cette base ;

8) devient visible au moment d'une défaillance : une infrastructure invisible quand elle fonctionne devient visible quand elle est défaillante : le serveur plante, le pont s'effondre, l'électricité est coupée. Même lorsqu'il y a des 
mécanismes ou des procédures de secours, leur existence ne fait que mettre en lumière l'infrastructure devenue visible.

La question est donc double. D'une part, il s'agit de savoir si nos observations de situations de gestion de crise entrent en résonnance avec la description de ces huit dimensions. D'autre part, il faut savoir si leur agencement, faisant infrastructure, se retrouve pour la gestion de crise. Cet agencement est plus précisément qualifié par Janet Vertesi de "moments d'alignement " permettant de concilier l'hétérogénéité des dimensions caractéristiques de l'infrastructure (Vertesi, 2014). De la même manière, les travaux de Kathryn Furlong et de Jane Bennett considèrent comme primordiale la notion d'assemblage pour déterminer ce qui fait infrastructure. K. Furlong affirme ainsi qu'il est " nécessaire d'aller au-delà de la conception d'infrastructure comme une entité unique, physiquement statique, aux effets sociaux et environnementaux figés. Comprendre l'infrastructure comme un assemblage de petites technologies permet d'envisager la possibilité de faire de petits changements pour traiter de grands problèmes $»^{2}$ (Furlong, 2010, p. 18). De son côté, J. Bennett utilise également la notion d'assemblage pour analyser la panne électrique nord-américaine de 2003 en insistant sur les relations de pouvoir qui s'y exercent. Pour elle, un assemblage est un réseau à topographie inégale : certains points où les trajectoires des actants se croisent sont plus densément utilisés que d'autres, et donc le pouvoir n'est pas distribué de façon égale sur le périmètre de l'assemblage. Elle ajoute aussi qu'un assemblage n'est pas gouverné par un pouvoir central : aucun membre n'a de compétence suffisante à lui seul pour déterminer en totalité les conséquences des activités de l'assemblage (Bennett, 2005 ; Bennett, 2009).

Toutefois, avant de confronter nos propres observations de terrain à la conception d'infrastructure développée dans les travaux précités, il est nécessaire d'expliciter ce que l'on entend par gestion (de crise). Valérie Boussard, par exemple, définit la gestion comme un ensemble de dispositifs mobilisés pour conduire le fonctionnement des organisations

2 Notre traduction de la citation suivante : « ... the need to look beyond infrastructure as a single unit, static in its physical state and social and environmental effects. Breaking infrastructure down into assemblages of small technologies that matter enables one to see the possibility to employ small change to mediate large problems. " 
(Boussard, 2008). Par extension, cette manière d'organiser quelque chose peut être associée, au-delà de l'action elle-même, au processus de mise en œuvre de cette action. Ainsi, la deuxième partie de ce chapitre propose de décrire nos observations pour voir précisément comment ce processus de mise en œuvre de l'action, caractéristique de la gestion de crise, s'ajuste aux éléments et à la dynamique intrinsèques à la notion d'infrastructure.

\section{Une infrastructure qui met temporairement en lumière un nouveau paysage...}

Lorsqu'une crise survient, c'est, dans un premier temps, le déploiement de l'infrastructure de la gestion de crise, qui offre au regard un nouveau paysage, faisant ressortir des éléments structurants, existant en soi, mais rendus alors explicitement visibles. Elle révèle ainsi l'existence de tout un réseau de nœuds, de flux et de zones.

Dans le cas d'une crise à cinétique rapide, générée par une attaque terroriste par exemple, les nouds ou points de rassemblement prennent d'abord la forme de points d'impact localisés dans l'espace et dans le temps. Il s'agit de lieux bien identifiés sur lesquels ont été perpétrées des attaques à un moment $t$. Ces nœeuds d'impacts entraînent dans un premier temps une convergence des forces de sécurité publique et de sécurité civile qui vont très rapidement structurer l'espace autour de ces points d'impact en zones concentriques. Une disposition générale prévoit ainsi la délimitation de trois zones contrôlées dans lesquelles la circulation va être limitée, voire strictement interdite. La première zone correspond à la zone d'exclusion. Réservée aux secours, aux forces de police et au déminage, elle délimite le premier cercle autour du point d'impact et par conséquent la zone de danger immédiat. Dans son prolongement est simultanément mise en place une zone tampon ou zone dite contrôlée, interdite aux médias et au public et réservée à la mise en place de secteurs de décontamination en cas d'attaque NRBC par exemple, et de façon plus générale au déploiement des intervenants en zone d'exclusion. Enfin, une troisième zone encercle les deux premières : la zone de soutien. Cette zone est la plus excentrée du point central. La circulation y est limitée et contrôlée. Elle permet d'accueillir les différents postes mobiles de commandement des opérations, les 
autorités et les élus, le Centre d'Accueil des Impliqués (CAI) ainsi qu'un centre de presse permettant de canaliser et d'informer les journalistes. Pour le citoyen qui n'est pas directement impliqué ou concerné par la crise qui se joue, ces différentes zones, matérialisées par des périmètres bien définis, correspondent à des espaces interdits, dans lesquels il n'est momentanément plus possible de circuler et qui supposent, en pratique, d'être contournés.

Dans un second temps, d'autres points cruciaux ou nœuds de rassemblements ayant pour vocation d'accueillir les victimes vont surgir pour devenir des éléments incontournables de l'infrastructure de gestion de crise. Il s'agit par exemple des hôpitaux ou des centres de soin.

Pour relier les premiers points d'impact à ces nœuds centraux de prise en charge des victimes, plusieurs grands axes vont alors émerger. Ces grands axes de circulation - routière principalement - vont être déterminés de deux façons : d'une part, selon la recherche du trajet le plus court possible reliant les points d'impact et les points d'accueil des victimes ; d'autre part, selon leur praticabilité, c'est-à-dire de façon à ce que les camions des sapeurs-pompiers, les véhicules du SAMU ou encore de la police puissent circuler sans entraves, de la façon la plus fluide possible. Ces axes de circulation mettent alors en exergue un nouveau maillage de la ville, au sein duquel d'autres axes, plus étroits, desservant l'échelle du quartier seront alors rendus moins visibles et momentanément plongés l'ombre. Dans le cas d'une crise à cinétique lente comme celle qui peut être générée par une crue majeure de la Seine à Paris, certains axes routiers seront soustraits à la circulation, de même que certains tronçons de lignes souterraines de RER ou de métro. En juin dernier, cela a été le cas avec la fermeture de la ligne intra-muros du RER C pendant toute la durée de la crue. Si la crue avait été plus importante encore, d'autres lignes de métro auraient été coupées de la circulation, reportant un important flux de passagers à la surface de la ville.

Le déploiement de l'infrastructure de gestion de crise redessine ainsi la ville tout en lui imposant un nouveau rythme : un afflux de population en certains endroits et a contrario une sorte de "vide » en d'autres endroits, l'affichage de panneaux de déviation, de panneaux alphanumériques ou de messages comportementaux relayant l'information sur la situation en cours et orientant ainsi les déplacements des habitants. 
En élargissant les sens qui nous permettent de percevoir un paysage, il est alors possible d'enrichir la description de ce passage de l'invisibilité à la visibilité des éléments constituant l'infrastructure de gestion de crise. L'ouïe peut par exemple prendre part à cette description. Les grands axes de circulation ressortant en temps de crise et décrits précédemment sont aussi matérialisés par le bruit des sirènes dont la fréquence s'intensifie, que ce soit le jour ou la nuit. Ainsi, le passage de l'invisibilité à la visibilité se fait aussi par une sorte d'uniformisation des rythmes journaliers, quel que soit le moment de la journée ou de la nuit. Par ailleurs, d'autres bruits inhabituels peuvent surgir : le bruit des hélicoptères d'urgence qui se déplacent hors des couloirs aériens et qui définissent alors d'autres axes de circulation surgissant uniquement en temps de crise avant de s'effacer en phase post-crise. Ainsi, le paysage urbain, révélé par la mise en place de l'infrastructure de gestion de crise, est un paysage de contrastes, fait de " trop-pleins " et de "vides ", de nouveaux trajets très empruntés et d'itinéraires temporairement inaccessibles, de concentration de bruits en certains endroits et de zones devenues subitement silencieuses en d'autres.

Dans le cas d'une crise liée à une crue majeure de la Seine, il est également possible d'ajouter à nos sens détecteurs, l'odorat. En effet, une crue génère tout un amas de détritus, obstrue des canalisations d'eaux usées, etc. Par conséquent, après la phase de décrue et de pompage des eaux stagnantes, se met en place une autre phase de nettoyage et de remise en état des réseaux de canalisations, souvent accompagnée d'odeurs nauséabondes et persistantes qui nous rappellent que l'on se trouve dans un paysage qui n'est pas habituel, aussi rendu appréhendable par des odeurs non habituelles.

Enfin, c'est aussi la description d'une ambiance, d'une atmosphère qui doit être prise en compte. Une ambiance façonnée par les médias classiques (télévisions et radios), mais aussi par les réseaux sociaux qui vont maintenir une population en alerte, la rendre particulièrement vigilante le temps de la crise et qui vont conférer une dimension supplémentaire à ce nouveau paysage de crise émergent. Initiées par des messages comportementaux ou par les médias, sociaux ou non, de nouvelles pratiques vont surgir : modification des pratiques récréatives, des déplacements par effet de contournement ou d'évitement. 


\section{... mais qui doit aussi se chercher dans l'ombre}

Toutefois, pour saisir la gestion de crise comme une infrastructure à part entière, il faut aller au-delà de la description du déploiement de l'infrastructure supportant cette gestion de crise. En d'autres termes, il ne faut pas se restreindre à ce qui est rendu directement visible $a$ priori. Il faut aussi essayer de prendre en compte, d'une part les éléments constitutifs de cette infrastructure qui n'arrivent pas à devenir visibles ou audibles pendant la crise, et d'autre part la façon dont ces éléments parviennent à s'assembler ou non. Le paragraphe suivant fournit quatre exemples observés pendant la gestion des crises liées aux attentats perpétrés en novembre 2015 et à la crue de la Seine de juin 2016.

Le premier exemple reprend la problématique liée à la mise en place du numéro vert lors des attentats survenus en novembre dernier. Lorsqu'un événement grave engendre un certain nombre de victimes, un numéro vert est systématiquement mis en place de façon à renseigner les familles et proches des victimes. Le déclenchement de ce numéro est le résultat d'une réflexion d'anticipation qui peut être considérée comme un élément constitutif de l'infrastructure de gestion de crise. Or sa mise en œuvre nécessite l'assemblage ou l'alignement de plusieurs facteurs.

En fonction de la situation, c'est-à-dire de sa gravité et de l'ampleur du nombre de victimes, le travail d'anticipation consiste à trouver le bon dimensionnement à donner à l'infrastructure. Pendant les attentats qui se sont perpétrés à Saint-Denis et à Paris en novembre 2015, certaines autorités ont rapidement compris, au regard du caractère hors-norme de l'événement, qu'il fallait adapter cette modalité de l'infrastructure de gestion de crise, de façon à l'ajuster aux besoins des appelants. Une proposition, consistant à trier les appels via l'affichage d'un bandeau sous le numéro vert, précisant que ce dernier était uniquement réservé aux familles et amis à la recherche de leurs proches, a été soumise. Or cette proposition n'a pas été entendue ni comprise au moment opportun, c'est-à-dire dès le début de la nuit du 13 au 14 novembre. Ainsi, l'ajustement du dimensionnement de l'infrastructure est resté invisible au lieu de devenir visible puisqu'aucun bandeau n'a finalement été associé au numéro vert. Sur ce point, notre infrastructure diffère des infrastructures classiques. La $8^{\mathrm{e}}$ dimension de l'infrastructure décrite 
dans les travaux de S.L. Star $e$ t al. indique que l'infrastructure devient visible au moment d'une défaillance. Dans notre cas, c'est l'impossible passage de l'invisible au visible qui rend défaillant tout un pan de notre infrastructure de gestion de crise. En effet, cette invisibilité maintenue a rapidement provoqué une saturation de la ligne téléphonique. Des retours d'expérience ont évalué, à un instant $t$ au cours de la nuit du 13 au 14 novembre, que le nombre d'appels enregistrés, était environ 100 fois supérieur au nombre total in fine de victimes. Beaucoup d'appels ne présentaient pas de caractère urgent et ne concernaient pas directement la recherche d'un proche. Or un certain nombre d'entre eux auraient certainement pu être redirigés via l'information délivrée par le bandeau associé au numéro vert. Par conséquent, les premières personnes concernées par la mise en place de ce numéro (familles et proches de victimes) n'ont pas systématiquement pu obtenir d'information sur la situation de leurs proches. Cet épisode a eu des conséquences directes sur le terrain puisque certaines familles ont alors entrepris de rechercher des informations par elles-mêmes, en se rendant physiquement dans les hôpitaux et centres de soin d'urgence susceptibles d'avoir pu les accueillir. Au-delà de la douleur psychologique et du stress qu'une telle démarche peut générer pour les familles, ces déplacements et regroupements spontanés ont contribué à engorger les hôpitaux et ajouté à la complexité de la prise en charge de victimes particulièrement meurtries.

Ainsi, l'une des caractéristiques de la gestion de crise envisagée comme infrastructure est la gestion du temps et en particulier la gestion délicate du passage de l'invisibilité à la visibilité. Une telle infrastructure correspond donc à la manifestation progressive, tout au long de la crise, de l'invisible au visible. Par ailleurs, la défaillance décrite ici résulte bien d'un non-alignement ou d'un assemblage qui n'a pas pu se faire au moment adéquat. Des divergences quant à l'appréciation de l'ampleur de cet événement hors norme n'ont pas permis de donner le bon dimensionnement à l'infrastructure de gestion de crise, entrainnant le blocage de la décision et par conséquent des répercussions directes sur le terrain. En ce sens, notre infrastructure de gestion de crise remplit les conditions d'alignement énoncées par J. Vertesi ainsi que la notion d'assemblage intrinsèque à celle d'infrastructure, développée par K. Furlong et J. Bennett. 
Le deuxième exemple observé concerne cette fois la gestion de la crue survenue en juin dernier. Lors de cet épisode de crue, le SGZDS a mis en œuvre sa principale mission qui consiste à anticiper, en fonction des besoins émis par les départements franciliens, la coordination des moyens zonaux, extrazonaux ou encore des renforts nationaux, mis à disposition par le Centre Opérationnel de Gestion Interministérielle de Crise (COGIC). Ces besoins sont d'abord définis par le terrain. Il faut ainsi attendre et anticiper le bon moment pour déployer les moyens permettant un pompage efficace. Si les moyens sont envoyés trop tôt, c'est-à-dire lorsque les surfaces inondées ne sont pas isolées en poches d'eau, mais encore reliées à un cours d'eau ou aux nappes phréatiques, alors leur pompage serait rendu totalement inefficace. Toutefois, au-delà de l'aspect opérationnel, dicté par l'évolution de la situation sur le terrain, d'autres facteurs doivent être pris en compte, et notamment le facteur financier. Tous les départements ne sont pas financés de la même façon et ne sont pas aussi riches les uns que les autres. Par conséquent, la mise en application d'un plan d'anticipation et de redistribution des moyens, élaboré uniquement à partir de la situation sur le terrain, ne fonctionnera pas nécessairement de la même façon lorsque celui-ci est mis en œuvre pour tel ou tel département, plus ou moins riche. En effet, l'État, via le COGIC, met à disposition des moyens nationaux. Le rôle du SGZDS est de coordonner ces moyens pour savoir comment les distribuer au bon moment et en quantité suffisante. Lorsqu'un département accepte un renfort de moyens nationaux, il doit en contrepartie assurer l'hébergement et la restauration des hommes venus en renfort. Dans certains cas, cet aspect financier peut parfois entrer en ligne de compte au point de contourner les renforts nationaux proposés lorsque la situation n'est pas critique et peut être résolue par des moyens propres, quitte à accepter un certain décalage temporel dans la résolution du problème. Cet exemple montre que le déploiement de l'infrastructure soutenant la gestion de crise ne suffit pas. Il faut aussi tenir compte de facteurs préexistants - en l'occurrence ici du facteur financier - indépendants de la situation de crise elle-même, mais pourtant incontournables à prendre en compte dans l'infrastructure de gestion de crise. En ce sens, cet exemple répond à la dimension trois de S.L. Star et al., à savoir la portée ou l'étendue de l'infrastructure qui va au-delà d'un événement isolé ou d'une pratique unique. 
Le troisième exemple, moins évident toutefois à faire émerger nettement, doit pourtant certainement être pris en compte : il s'agit $\mathrm{du}$ facteur politique. Dans le cas d'une crise touchant un territoire circonscrit à une zone de défense et de sécurité, le préfet de zone coordonne et déploie les moyens départementaux, puis rend compte des décisions prises à l'échelon supérieur, c'est-à-dire au ministère de l'Intérieur, représenté par le COGIC. En revanche, si la crise s'étend au-delà de l'échelle zonale, alors l'un des volets du dispositif ORSEC (Organisation de la Réponse de Sécurité civile) prévoit la possibilité pour les départements sinistrés de demander des renforts supplémentaires. Cette demande de moyens est transmise à la Zone de Défense qui la répercute au COGIC, puis transmet aux départements demandeurs la réponse de ce dernier. Selon la gravité et l'ampleur de la crise, la chaîne de commandement peut également s'étirer à l'échelon interministériel en armant la CIC (Cellule Interministérielle de Crise).

Ainsi, quelle que soit l'ampleur de la crise, la Zone de Défense apparaît comme un maillon essentiel de la chaîne de commandement, situé à l'articulation entre gestion opérationnelle et gestion stratégique de la crise. La position du préfet de zone lui permet donc d'avoir à la fois une vision globale stratégique et une vision fine du terrain. Par conséquent, cette position intermédiaire confère à la Zone de Défense la capacité de prévoir et d'honorer des demandes en fonction de l'évolution de la situation. Pourtant, la pression de la volonté politique peut, à certains endroits, rompre cette chaîne de commandement. Dans son ouvrage Une catastrophe ordinaire, relatif à la tempête de 1999, François Dedieu mentionne ce facteur politique sous l'expression " connexion personnelle ". Autrement dit, lorsqu'un préfet de département " contacte le secrétaire d'État du ministère de l'Intérieur qu'il connaît personnellement pour l'avoir régulièrement côtoyé dans son précédent poste à la direction des routes du ministère de l'Équipement " (Dedieu, 2013, p. 135), la demande de moyens est très vite honorée, et ce sans passer par la Zone de Défense ni le COGIC. Si cette démarche de contournement a bien permis ici d'accélérer le déploiement de moyens, on ne peut pourtant pas s'empêcher d'imaginer la confusion provoquée pour la Zone de Défense si celle-ci comptait utiliser autrement les moyens octroyés d'office. Par ailleurs, dans le cas cité par F. Dedieu, la demande provenait du bas de la chaîne de commandement : alors 
qu'elle ne respectait pas la hiérarchie, elle correspondait néanmoins à un réel besoin émanant du terrain. Mais lorsque la demande de moyens provient directement de l'échelon interministériel, celle-ci est a priori déconnectée du terrain : elle ne correspond plus à un besoin réel, mais à une volonté politique soutenue éventuellement par une pression médiatique de s'assurer que tel département ou telle commune, choisie pour des raisons de " connexion personnelle ", ne manquera de rien. Là encore, outrepasser les compétences de la Zone de Défense peut provoquer une confusion conduisant à une action inefficace. En l'occurrence, déployer des moyens de pompage alors que le secteur inondé n'est pas encore isolé, mais toujours alimenté par une nappe phréatique ou un cours d'eau en débordement, est inefficace. Dans le cas présent, cette inefficacité a pu être mentionnée suffisamment tôt pour éviter de se fourvoyer totalement, mais la perte de temps liée à la gestion de cette demande non pertinente mobilise inutilement des forces de travail qui auraient pu être mises au service d'autres problèmes cruciaux à résoudre au même moment. Ainsi, alors qu'initialement l'objectif de cette pression politique était à l'évidence de faire accélérer les choses, la conséquence du contournement des échelons hiérarchisés de la chaîne de commandement a généré l'effet exactement inverse.

Enfin, le dernier exemple relève de la responsabilité juridique et concerne les protocoles de réintégration d'un bâtiment public après inondation. Lorsque cette question de responsabilité a été posée en entretien à froid, plusieurs semaines après la gestion de la crue de la Seine en juin dernier, le discours, côté partenaires ou côté Secrétariat Général de la Zone de Défense et de Sécurité, est apparu homogène et énoncé comme une évidence : si ce n'est pas le responsable d'établissement qui décide de l'évacuation, alors c'est le préfet de département qui en a la responsabilité. La décision de réintégration du bâtiment, après la crue, revient alors à celui qui a pris la décision de faire évacuer, de façon à maintenir une continuité de la décision. Pourtant, l'observation de la gestion de la crue depuis le Centre de Crise Zonal a montré une tout autre situation. À partir du moment où les services de Vigicrue ont été en mesure d'annoncer quand et à quelle hauteur le pic de crue serait atteint à Austerlitz, les objectifs des partenaires ont été simultanément redéfinis. Chacun a réaiguillé le cap pour atteindre un retour à la normale le plus rapide possible. Or, parallèlement à ce basculement vers la gestion de la 
décrue, la question de la responsabilité juridique a émergé nettement et est devenue prioritaire. Plus précisément, un partenaire a contacté la Zone de Défense pour savoir s'il existait des fiches types sur les mesures à mettre en œuvre pour réintégrer un bâtiment. Dans l'absolu, après une inondation, il faut d'abord pomper l'eau puis nettoyer les lieux avant de faire intervenir un expert en bâtiment à même de vérifier la solidité du bâtiment et d'assurer, en toute sécurité, sa remise en fonctionnement (réactivation des différents réseaux électriques, d'eau, etc.) puis sa réintégration. Or l'attribution de cette responsabilité juridique ne semble pas toujours évidente pour les différents acteurs de la gestion de crise, qui, en situation de stress peuvent avoir tendance à ne pas vouloir engager leur propre responsabilité. Qui est responsable juridiquement ? À quel expert faire appel ? Par qui est-il nommé ? Face à ces questions, la Zone de Défense n'a pas souhaité s'engager plus avant, partagée entre son rôle de soutien aux partenaires et le souci de se protéger juridiquement. Cette observation rejoint les propos de F. Dedieu sur la recherche d'une protection juridique par les organismes impliqués en cas de crise en prenant l'exemple de Météo France : « [...] en raison de son statut d'organisme public, Météo France est formellement assujetti à une obligation de "moyens" et non à une obligation de "résultat". Ce principe juridique, inscrit dans les statuts de Météo France, prévoit que "tous les moyens dont disposent les organismes de Météo France doivent être mis en œuvre" dans la production de la prévision " (Dedieu, 2013, p. 35). Autrement dit, lorsqu'un doute subsiste, l'organisme public va se retrancher derrière son obligation de moyens. C'est ce qui s'est passé pour la Zone de Défense, qui a joué un rôle de conseil en suggérant à ce partenaire de faire intervenir un architecte de leur service pour évaluer la possible réintégration du bâtiment, sans donner directement son accord. Cette attitude correspond à une projection vers l'après-crise et la peur d'être condamné, qui, selon F. Dedieu, est fréquente : " Après de grandes catastrophes, on observe souvent une tendance critique de la vulnérabilité, qui consiste à ne pas se contenter de révéler de manière neutre les limites des systèmes à appréhender certains risques, mais davantage à rechercher des manquements, des fautes ou encore des coupables " (Dedieu, 2013, p. 15). Ainsi, alors qu'en phase de gestion de la crue, c'est-à-dire avant la stabilisation de la situation de crise, les objectifs des uns et des autres convergent vers un même but, l'amorce de la phase de décrue et l'objectif pourtant partagé du retour à la normale, 
tend à diviser les forces : la volonté de se protéger juridiquement devient cruciale, prioritaire et nettement visible. Le facteur juridique apparaît donc comme un élément incontournable de l'infrastructure de gestion de crise. Néanmoins, les causes de sa manifestation posent question puisqu'elles tendent à entraver la réalisation d'un objectif pourtant commun à l'ensemble des acteurs impliqués dans la crise : le retour à la normale le plus rapide possible. Cet exemple confirme que l'infrastructure de gestion de crise dépasse la stricte durée de la crise. Ainsi, le besoin d'alignement ne peut se restreindre à un moment $t$, mais cet alignement doit aussi se faire de façon longitudinale, en tenant compte des conséquences à moyen et long termes d'une décision prise à un moment $t$, relevant de la gestion de crise. Sans cela, l'assemblage ne peut se faire et la gestion de crise se paralyse au point de pouvoir générer une " mini- "crise dans la crise. La non-réintégration en temps voulu de bâtiments de services publics, tels les hôpitaux par exemple, peut avoir de lourdes conséquences.

Ces quatre exemples, tirés d'observations directes, nécessitent d'être replacés dans une discussion plus large afin d'expliciter l'ensemble des facteurs à prendre en compte et leur enchevêtrement pour dessiner précisément les contours de l'infrastructure de gestion de crise et notamment la question de sa maintenance en dehors des situations de crise.

\section{Discussion et conclusion}

D'après les exemples évoqués supra, il est clair que la plupart des dimensions de l'infrastructure mises en évidence par S.L. Star et al., sont présentes dans la gestion de crise. Celle-ci est en effet totalement " coulée " à l'intérieur d'autres structures, arrangements sociaux et technologies (électricité, hôpitaux, chaîne de commandement, numéro $117 \ldots$ ) (dimension 1) ; elle est également transparente pour l'usager (dimension 2) car l'organisation des secours prend en charge la situation - dans la mesure de ses possibilités. La gestion de crise a une portée spatiale et temporelle liée à la situation (l'ampleur des moyens mis en ouvre et à disposition va varier constamment au cours de la crise) et n'est pas une pratique unique ni un événement isolé (dimension 3). Gérer les crises suppose de connaître les codes (la chaîne 
de commandement, ou encore la manipulation des outils collaboratifs de gestion de crise, par exemple) et développer un vocabulaire spécifique (rédaction de points de situation par exemple), ce qui correspond à la dimension 4. Ce dernier point est en lien aussi avec les nombreuses conventions pratiques et l'incorporation de normes et de standards qui existent dans la gestion de crise (dimensions 5 et 6). La dimension 7 est également présente en gestion de crise puisque l'infrastructure ne vient pas de rien. Elle est construite sur une base installée, à savoir l'organisation des premiers secours urbains, les sapeurs-pompiers au départ, puis la police très rapidement, qui ont été de tout temps les gardiens de l'ordre public, de la sécurité et de la salubrité. Toutefois, comme le mentionnent Star et al. (1996), une infrastructure nécessite un effort considérable pour lutter contre l'inertie de la base installée. La coordination de crise est à ce prix, elle est souvent insatisfaisante, toujours en perpétuelle amélioration. Finalement, on pourrait dire que l'effort de maintenance de la gestion de crise (donc de cette infrastructure) est très important. Elle s'entretient notamment à force d'exercices réguliers de plus ou moins grande ampleur (cf. l'exercice européen Sequana du mois de mars 2016), où divers partenaires, souvent hétérogènes et agissant à des échelles territoriales diverses, simulent des situations de crise et collaborent pour la résoudre au mieux. La question qui se pose est celle du (peu) d'importance que l'on accorde à cette maintenance en raison de son coût et de la mesure extrêmement difficile de son efficacité : une gestion de crise jugée efficace par les intervenants - c'est-à-dire se déroulant de façon fluide et sans heurts - n'est pas remise en cause par les autorités, mais n'est pas non plus valorisée. Or nous suivons en cela Steve Graham et de Nigel Thrift qui montrent à quel point la maintenance et the repair sont importants pour comprendre les sociétés modernes et en particulier les villes (Graham et Thrift, 2007). La maintenance est de fait un thème à valoriser, central pour notre objet, et pose la difficile question de savoir comment maintenir et améliorer un assemblage qui n'est activé que de façon discontinue.

Enfin - et c'est sans doute à cet endroit particulier (dimension 8) que notre infrastructure de gestion de crise diffère légèrement de l'infrastructure décrite par S.L. Star et al. -, notre infrastructure ne présente pas systématiquement de défaillance lorsqu' elle devient visible. 
Au contraire même, c'est lorsque le passage de l'invisible au visible ne se fait pas au moment opportun, que notre infrastructure révèle un dimensionnement inadéquat.

Un dernier point de discussion doit être soulevé, celui du lien entre infrastructure de gestion de crise et urbanité. Le caractère urbain d'un territoire peut se définir par sa densité et sa diversité (Lussault, in Lévy et Lussault, 2003), sources de complexité. Cette complexité se lit dans la notion d'assemblage observée dans nos exemples. Par conséquent, en considérant la façon dont les assemblages se font ou ne se font pas, il est possible de relier infrastructure de gestion de crise et urbanité. La complexité du territoire urbain renforce l'ampleur de la crise en décuplant ses conséquences directes et indirectes. Elle permet donc aussi de mieux mettre en exergue les éléments visibles du paysage urbain révélé en cas de crise. En ce sens, le milieu urbain exacerbe les caractéristiques ou dimensions de l'infrastructure pensée comme gestion de crise.

Par ailleurs, l'exemple de la métropole parisienne est particulier puisque c'est la seule partie du territoire français sur laquelle sont représentés tous les échelons territoriaux de la chaîne de commandement, depuis l'échelon de la commune jusqu'à l'échelon interministériel. Ce territoire concentre donc l'ensemble des niveaux de cellules qui peuvent être activés en cas de crise.

Ainsi, la question urbaine est bien présente dans notre conception de l'infrastructure puisqu'elle distingue des échelons territoriaux. En revanche, cette conception de l'infrastructure mériterait encore d'être développée plus avant pour savoir si elle peut être qualifiée de territorialisée. En somme, la spécificité du milieu urbain peut-elle/ doit-elle devenir une condition nécessaire à sa matérialisation ? Peutelle figurer comme dimension supplémentaire à celles énumérées par S.L. Star et K. Ruhleder (2010) pour savoir à partir de quand on est en présence d'une telle infrastructure?

\section{Références}

Bennett J. (2005), "The Agency of Assemblages and the North American Blackout », Public Culture, n 17 , p. 445-465. 
Bennett J. (2009), Vibrant matter: A political ecology of things, Durham, NC, Duke University Press.

Boin A., McConnell A. (2007), " Preparing for Critical Infrastructure Breakdowns: The Limits of Crisis Management and the Need for Resilience ", Journal of Contingencies and Crisis Management, $\mathrm{n}^{\circ} 15$, p. $50-59$.

Boin A., Smith D. (2006), "Terrorism and critical infrastructures: Implications for public-private crisis management ", Public Money and Management, $\mathrm{n}^{\circ}$ 26, p. 295-304.

Boussard V. (2008), Sociologie de la gestion. Les faiseurs de performances, Paris, Belin.

Dedieu F. (2013), Une catastrophe ordinaire. La tempête du 27 décembre 1999, Paris, EHESS.

Furlong K. (2010), " Small technologies, big change: Rethinking infrastructure through STS and geography ", Progress in Human Geography, p. 1-23.

Galland J.-P. (2010), "Critique de la notion d'infrastructure critique ", Flux, no 81, p. 6-18.

Graham S., Thrift N. (2007), "Out of Order: Understanding Repair and Maintenance ", Theory, Culture \& Society, n² 24, p. 1-25.

LaPorte T. R. (2007), "Critical Infrastructure in the Face of a Predatory Future: Preparing for Untoward Surprise ", Journal of Contingencies and Crisis Management, $\mathrm{n}^{\circ} 15$, p. 60-64.

Lave J., Wenger E. (1999), "Learning and pedagogy in communities of practice ", Learners and Pedagogy, p. 21-33.

Lévy J., Lussault M. (2003), Dictionnaire de la géographie et de l'espace des sociétés, Paris, Belin.

Mendonça D., Wallace W. A. (2006), "Impacts of the 2001 world trade center attack on New York City critical infrastructures ", Journal of Infrastructure Systems, $\mathrm{n}^{\circ}$ 12, p. 260-270.

OCDE (2003), Emerging Risks in the 21st Century, ed. O. I. F. Program, 16.

Rostum J., November V., Varn J. (2008), « Proactive Crisis Management of Urban Infrastructure ", COST 19 Report Urban Civil Engineering Bruxelles, European Science Foundation. 
Star S. L. (1991), "The sociology of the invisible: The primacy of work in the writings of Anselm Strauss ", dans Maines D. R. (éd.), Social organization and social process: Essays in honor of Anselm Strauss, New York, A. de Gruyter, p. 265-283.

Star S. L., Bowker G. C. (2006), "How to infrastructure ", dans Lievrouw L. A., Livingstone S. (éds), Handbook of new media: Social shaping and social consequences of ICTs, Londres, Sage, p. 230-245.

Star S. L., Ruhleder K. (1996), "Steps toward an ecology of infrastructure: Design and access for large information spaces ", Information Systems Research, $\mathrm{n}^{\circ}$ 7, p. 111-134.

Star S. L., Ruhleder K. (2010), "Vers une écologie de l'infrastructure ", Revue d'anthropologie des connaissances, vol. 4, n 1, p. 114-161.

Therrien M.-C. (2010), "Stratégies de résilience et infrastructures essentielles ", Télescope, vol. 16, n 2, p. 154-171.

Vertesi J. (2014), "Seamful spaces: Heterogeneous infrastructures in interaction ", Science, Technology \& Human Values, vol. 39, n² 2, p. 264284. 


\section{Le processus d'infrastructuralisation de la télémédecine et de la télésanté}

\section{Vers une endogénéisation des dispositifs dans les standards existants?}

\section{Alexandre Mathieu-Fritz}

Le recours à la notion d'infrastructure permet de penser sous un angle original certains des principaux changements qui sont aujourd'hui à l'œuvre au sein des mondes de la médecine et de la santé qui, à l'instar d'autres mondes techniques, sont marqués par l'avènement du numérique et l'intégration croissante des technologies de l'information et de la communication (TIC). Le développement de la télémédecine et de la télésanté ${ }^{1}$ constitue une expression particulière de ces mutations et repose sur diverses infrastructures, qu'elles soient institutionnelles ou techniques. Les dispositifs de télémédecine se caractérisent par un double phénomène d'infrastructuralisation : d'une part, divers processus concourent conjointement à leur stabilisation, leur pérennisation et leur extension en leur offrant une base solide de développement, un soubassement; d'autre part, un grand nombre de dispositifs de télémédecine et de télésanté vont jusqu’à se fondre dans les standards et les dispositifs des infrastructures existantes, dont la prise en compte nous permet de réinterroger les usages des dispositifs étudiés et de mieux mettre en lumière certaines de leurs particularités.

La télémédecine consiste à pratiquer la médecine à distance du patient grâce aux TIC. La téléconsultation et la téléexpertise constituent les deux principaux actes de télémédecine. La première consiste à réaliser avec le patient une consultation à distance et la seconde à demander un avis à un médecin spécialiste qui se prononcera sur la base d'un dossier contenant des informations cliniques. La télésanté englobe plus largement tous les sites Internet et les applications qui ont trait à la santé. 
Notre programme de recherche sur le développement de la télémédecine et de la télésanté s'inspire de la perspective interactionniste d'Everett C. Hughes (1996) et d'Anselm L. Strauss (1992). Il a pour objectif principal d'appréhender les effets des usages des dispositifs de téléconsultation sur les interactions entre professionnels de santé et usagers, sur les pratiques, les savoirs et les identités professionnels, ainsi que sur la division du travail et la dynamique des groupes professionnels. Ce programme de recherche repose sur l'exploitation de plusieurs terrains d'enquête. Le premier est celui du dispositif de télémédecine Télégéria qui met en lien deux structures de soins d'un même pôle hospitalier, un $\mathrm{CHU}$ et un hôpital gériatrique. Son objectif est de proposer des téléconsultations dans plusieurs spécialités médicales à des personnes âgées séjournant à l'hôpital gériatrique tout en leur évitant les déplacements en ambulance entre les deux sites. Le second travail de terrain a eu lieu dans le cadre d'un réseau de psychiatres et de psychologues cliniciens (Télé-med) proposant via un site Internet des téléconsultations à des patients français expatriés.

\section{Les dispositifs et les réseaux de télémédecine et de télésanté ne constituent pas des infrastructures}

Par " infrastructure ", nous faisons référence, en nous inspirant des travaux menés par Paul N. Edwards, Thomas P. Hughes, Geoffrey C. Bowker et Susan L. Star', aux grands systèmes sociotechniques stabilisés, durablement installés, dont les principales caractéristiques sont relativement pérennes et donc difficilement (ou coûteusement) réversibles et dont le degré d'extension territoriale est très important, touchant ainsi potentiellement, de façon directe ou indirecte, la plupart des acteurs sociaux. Les infrastructures peuvent être étudiées à différentes échelles, de façon combinée ou non. Elles constituent le nécessaire soubassement des dispositifs sociotechniques ${ }^{3}$ les plus répandus et ont pour caractéristique d'être invisibles lorsqu'elles fonctionnent normalement, c'est-à-dire sans défaillance.

2 Voir notamment Edwards (2003), Edwards et al. (2009), Hughes (1999), Bowker et Star (1999).

3 Les dispositifs, dont le fonctionnement dépend pour une large part des infrastructures existantes, "sont des ensembles de repères objectivés, stabilisés, qui prépar- 
Nous parlerons d'infrastructuralisation pour désigner le processus de développement de vastes dispositifs sociotechniques présentant, suivant des niveaux d'achèvement plus ou moins élevés, certains traits caractéristiques des infrastructures. Fondamentalement, ce terme désigne ainsi la constitution de ce qui sous-tend ce développement, c'est-à-dire un soubassement. Nous verrons aussi que la notion d'infrastructuralisation peut prendre un second sens et nous conduire à mettre en lumière un aspect particulier de nos objets de recherche, à savoir celui de l'endogénéisation d'innovations ou de nouveaux dispositifs techniques dans les infrastructures existantes (comme Internet ou le réseau de téléphonie mobile). L'endogénéisation doit être comprise comme le fait qu'un nouveau dispositif technique vient se fondre pour une part plus ou moins importante dans les standards des infrastructures existantes ${ }^{4}$.

Actuellement, les réseaux et les dispositifs de télémédecine et de télésanté observés ne constituent pas des infrastructures en raison de leur petite taille, du degré d'instabilité de leurs caractéristiques et de leur faible durabilité. Il y a en effet, tout d'abord, une question d'échelle à prendre en compte. En télémédecine, notamment, les réseaux qui fonctionnent sont généralement de taille réduite, c'est-à-dire qu'ils ne comptent pas plus d'une dizaine de " points "-i.e. de sites distants, équipés et reliés. Par ailleurs, environ la moitié des expérimentations qui sont financées ne sont pas fonctionnelles, en raison principalement de problèmes d'organisation et d'usage, les responsables des projets ayant tendance à sous-estimer le coût organisationnel de la mise en place des dispositifs de télémédecine et à occulter le travail réel des praticiens, qui connaît généralement d'importantes transformations à l'occasion

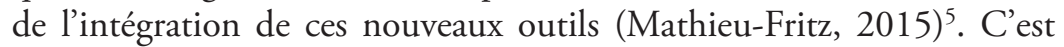
certainement pour cela que la plupart des pionniers de la télémédecine insistent sur la nécessité de " commencer petit ».

ent et guident les jugements. Ils sont disponibles sous forme de mots, de règles et d'objets" (Dodier, 1993).

4 Nous préférons ce terme à celui d'encastrement, qui est utilisé notamment par Susan L. Star, car la notion d'endogénéisation permet de mieux signifier qu'il s'agit d'une tendance générale (qui souffre donc des exceptions), mais aussi d'un processus dont il importe d'apprécier le degré de développement et d'extension.

5 Le cas de l'échec du dossier médical personnel français sur le plan de son utilisation illustre de façon éclatante ce problème (Mathieu-Fritz et Esterle, 2013). 
Un autre critère de définition d'une infrastructure n'est pas rempli : il s'agit de la stabilisation des caractéristiques des dispositifs. Ces derniers évoluent à la fois sur le plan technique et au niveau de leurs usages, notamment lors des phases expérimentales des projets de télémédecine où les coordonnateurs ont tendance à traduire dans les dispositifs les demandes des utilisateurs (potentiels) afin d'augmenter leurs chances de voir ces derniers s'y investir. Par exemple, le médecin coordonnateur de Télégéria (Mathieu-Fritz et Esterle, 2013a et 2013b), suivant la demande du professeur de cardiologie participant au projet, a décidé de procéder à des transformations du dispositif technique afin d'augmenter la fréquence de transmission des images issues de l'échographe pour pouvoir pratiquer des échographies cardiaques avec le même rendu visuel qu'en co-présence. Répondant à la demande d'autres utilisateurs, il a ajouté un robot permettant de faire bouger la caméra mobile du dispositif à l'aide d'un petit joystick - et donc sans recourir à l'aide d'une personne située aux côtés du patient - et a amélioré la restitution sonore, en faisant poser de nouveaux micros dans la salle de téléconsultation. Il a aussi ajouté un écran supplémentaire aux deux écrans existants, ce qui permet de voir le patient, d'observer l'image de la caméra mobile et de consulter le dossier médical de ce dernier sur des écrans distincts. D’un point de vue général, les dispositifs de télémédecine sont loin d'être standardisés. On observe en effet d'importantes variations sur le plan de la technologie utilisée, des objectifs initiaux des projets, c'est-à-dire de leur périmètre - ou champ d'action - médical (quelle(s) pathologie(s) traite-t-on à distance ?), du nombre des acteurs concernés et des catégories de professionnels impliquées, du nombre de sites distants et de leurs caractéristiques, ainsi que du temps consacré au pilotage.

Enfin, la plupart des dispositifs n'ont pas la pérennité d'une infrastructure et demeurent, aujourd'hui encore, très dépendants du degré d'engagement personnel des acteurs qui coordonnent les projets. La coordination des projets de télémédecine constitue une tâche protéiforme qui requiert de nombreuses compétences et qui est particulièrement chronophage (Mathieu-Fritz et al., 2011). Lorsque le porteur du projet interrompt son activité, c'est bien souvent le dispositif qui cesse de fonctionner. 
En bref, nous pourrons parler d'infrastructures en télémédecine lorsque ses réseaux et ses dispositifs couvriront l'ensemble des territoires de santé de façon pérenne, touchant ainsi potentiellement un grand nombre d'individus, en présentant des caractéristiques de fonctionnement et d'utilisation relativement stables et standardisées. Mais tel n'est pas encore le cas aujourd'hui, tant s'en faut ${ }^{6}$.

\section{L'infrastructuralisation de la télémédecine et de la télésanté par endogénéisation des dispositifs et des usages}

Les constatations précédentes ne doivent pas nous conduire à occulter la dimension infrastructurelle des dispositifs étudiés. Remarquons tout d'abord que le fonctionnement des dispositifs et des réseaux de télémédecine et de télésanté dépend des infrastructures existantes à divers titres. Le fonctionnement d'une grande partie d'entre eux repose en effet sur des infrastructures techniques " déjà là », comme le réseau électrique, Internet ou les réseaux Intranet, tels ceux qui relient plusieurs établissements d'un même pôle hospitalier. On peut ajouter à ces infrastructures celles de la téléphonie mobile, dont le réseau est utilisé pour développer tout un ensemble d'applications en télésanté et en télémédecine - e.g. en cardiologie pour la télésurveillance et en diabétologie pour un suivi quotidien adapté 7 . De manière analogue, les dispositifs de visiophonie et de téléprésence existaient avant d'être intégrés à ceux de la télémédecine ou de la télésanté.

De telles considérations appellent une remarque importante. C'est le caractère massif et la fréquence élevée d'utilisation, ainsi que la routinisation des usages et des modes de coopération qui les accompagnent - autant de phénomènes qui se développent de façon progressive - qui font qu'il est difficile de se passer des infrastructures, autrement dit que celles-ci se stabilisent de façon relativement durable. Nous ne cherchons donc pas à développer une perspective concevant un déterminisme technique où les infrastructures s'imposeraient de façon nécessaire.

7 Ainsi, dans un pays comme la Corée du Sud, la couverture du réseau 4G à l'échelle nationale rend possibles des projets de télésurveillance cardiaque dans le cadre desquels le téléphone portable joue le rôle de borne wi-fi, permettant de transmettre en temps réel les relevés cardiaques des usagers. 
La dépendance des dispositifs de télémédecine à l'égard des infrastructures semble mieux mise en lumière si l'on prend en compte le fait qu'un processus d'infrastructuralisation de la télémédecine est à l'œuvre. Dans un premier sens, cela signifie que les soubassements de ses dispositifs, qui concourent à leur stabilisation, leur pérennisation et leur extension, sont en cours de constitution. Ce processus d'infrastructuralisation est alimenté, depuis la première moitié des années 2000, par la mise sur l'agenda politique de la télémédecine, par le processus de rationalisation juridique dont elle fait l'objet, par le développement par des industriels de nouveaux dispositifs et outils communicants, et au sein de la profession médicale, par l'existence de pionniers développant - initialement, avec de modestes moyens - des projets de télémédecine et par les actions menées par la société française de télémédecine, la SFT-Antel, auprès de diverses instances étatiques.

Les fondements juridiques de la télémédecine sont en cours d'élaboration depuis la loi de 2004 relative à l'assurance maladie, qui reconnaît la pratique de la télémédecine sans toutefois lui donner une définition détaillée 8 . Cinq ans plus tard, cette reconnaissance se confirme et se précise par l'article 78 de la loi « Hôpital, patients, santé, territoires " (HPST) du 21 juillet 2009, dont les règles de mise en œuvre sont précisées dans le décret du 19 octobre 2010. Loi et décret sont désormais inscrits dans le Code de la santé publique ${ }^{9}$. Le développement de la télémédecine en France repose sur une stratégie nationale définie en conseil des ministres le 8 juin 2011 , lequel a arrêté 5 priorités nationales ${ }^{10}$ qui sont déclinées au plan local par les agences régionales de santé (ARS) dans un programme obligatoire régional de télémédecine. La stratégie nationale de déploiement de la télémédecine est pilotée par la Direction générale de l'offre de soins (DGOS). À l'échelle régionale, les ARS ont pour mission de contractualiser avec les promoteurs de projets afin de vérifier que ces derniers sont bien conformes aux conditions de mise

\footnotetext{
8 Nous faisons référence ici à la loi nº 2004-810 du 13 août 2004 relative à l'assurance maladie.

9 Article L. 6316-1 du Code de la santé publique.

10 Ces 5 priorités sont la permanence des soins en imagerie médicale, la prise en charge des accidents vasculaires cérébraux (AVC), la santé des personnes détenues, la prise en charge d'une maladie chronique comme l'insuffisance rénale chronique, l'insuffisance cardiaque, le diabète, etc., et, enfin, les soins en structure médicosociale ou en hospitalisation à domicile (HAD).
} 
en œuvre décrites dans le décret. Le développement de la télémédecine sur les territoires est désormais encadré juridiquement suivant un double niveau de contractualisation liant les différents acteurs des projets. Les ARS peuvent, si nécessaire, apporter un soutien financier, notamment pour favoriser le développement de la télémédecine dans le secteur ambulatoire, la télémédecine hospitalière étant généralement financée par la tarification à l'activité (T2A). Aujourd'hui, les actes de télémédecine constituent des actes médicaux et leur tarification est mise en place dans des régions-pilotes à titre expérimental avant d'être appliquée, vraisemblablement par disciplines médicales, à l'ensemble du territoire national. La tarification des actes de télémédecine actuellement en cours d'élaboration peut être envisagée pour une part comme un processus de conformation aux standards de l'Assurance-maladie l'objectif étant le remboursement des actes de télémédecine ${ }^{11}$.

L'infrastructuralisation de la télémédecine se caractérise ainsi par un processus de rationalisation juridique et administrative visant à définir et encadrer les pratiques ainsi que l'utilisation des ressources. Ce processus peut être défini comme une forme d'institutionnalisation, dans la mesure où des règles de droit sont établies et des organisations mises en place afin de remplir ces objectifs. L'infrastructuralisation de la télémédecine est ainsi à la fois de nature juridique, économique et organisationnelle (ou institutionnelle). Les ARS constituent l'espace institutionnel à partir duquel se négocient les projets de télémédecine et où les stratégies et les projets développés préalablement par diverses catégories d'acteurs du monde de la santé sont exprimés, se rencontrent, se heurtent, entrent en tension et en conflit, mais aussi se négocient et se combinent. Ces instances peuvent être envisagées comme un point de rencontre, où se cristallisent et se combinent des stratégies respectivement bottom-uptelles qu'elles sont portées, localement, par des médecins développant des projets de télémédecine reliant diverses structures de soins - et top-down - i.e. des stratégies concernant davantage les objectifs de la télémédecine tels qu'ils sont formulés au niveau politique national et surtout au niveau régional de l'ARS, qui peut contribuer à impulser des projets de télémédecine et à leur donner une forme spécifique.

11 Dans certains cas, le non-remboursement des actes de télémédecine constitue une entrave au développement de la télémédecine elle-même (notamment du côté de la médecine de ville). 
Au-delà de l'idée de constitution d'un soubassement, d'une base offrant la possibilité d'un développement à venir, la notion d'infrastructuralisation peut aussi prendre un second sens et désigner un processus qui consiste en une endogénéisation de nouveaux dispositifs dans des infrastructures existantes. La notion d'endogénéisation nous permet de mettre en relief le fait que de nouveaux dispositifs médicaux ou de santé, mais aussi de nouveaux usages et de nouvelles techniques professionnelles et profanes viennent se fondre pour une large part dans les standards des infrastructures existantes. Naguère, les activités médicales s'effectuaient principalement à l'aide d'outils médicaux ${ }^{12}$, c'est-à-dire d'outils spécifiquement consacrés à un usage médical ; aujourd'hui, des outils et des dispositifs techniques qui ne sont pas conçus initialement pour être utilisés dans le cadre de la pratique médicale donnent lieu à des usages médicaux. Un grand nombre de réseaux de télémédecine fonctionnent en effet, au plan technique, en mobilisant des technologies standards, destinées initialement au grand public, à savoir un ordinateur portable, une connexion Internet et une webcam offrant des conditions d'interaction à distance similaire à celle de la visiophonie par Internet médiée par des logiciels comme Skype. De façon analogue, des tablettes (tels les iPad) sont utilisées comme caméra mobile en télédermatologie. En pareil cas, le dispositif technique (tablette, réseau wi-fi, connexion Internet) est utilisé de la même façon que pour la relation visiophonique classique, mais dans un cadre médical. D’autres dispositifs, qui reposent également sur les technologies existantes, sont plus évolués sur le plan technique que la visiophonie traditionnelle et offrent un cadre d'interaction similaire à celui de la téléprésence où les conditions d'observabilité, d'audibilité et, finalement, d'interaction sont très proches de celles des relations face-à-face : on voit son interlocuteur en grandeur réelle ou presque, les restitutions visuelle et sonore s'effectuent en temps réel et garantissent la fluidité des échanges (Relieu, 2007 ; Bonu, 2007, p. 50).

La notion d'endogénéisation invite à regarder de près les usages et les modes d'appropriation des dispositifs existants, afin de voir dans quelle mesure les outils, en fonction de leurs caractéristiques propres, mais également selon le contexte d'utilisation, les savoirs et l'expérience

12 Les usages du téléphone constituent une exception notable à ce constat. 
détenus par les utilisateurs, présentent à la fois des contraintes et des opportunités, offrent des marges d'action à ces derniers tout en contribuant à transformer leurs pratiques.

Pour tenter d'éclairer ce dernier aspect, nous pouvons évoquer le cas d'une application récente en télédiabétologie mise en place en phase expérimentale par des industriels proposant de petits dispositifs connectés qui consistent en une application mobile à informer quotidiennement. Cette application propose un suivi personnalisé basé sur les données fournies par le patient (glycémie, quantité de glucides prévue pour le repas, activité physique) et calcule instantanément la dose d'insuline qui lui est nécessaire ${ }^{13}$. À cet égard, si l'expérience des maladies chroniques - comme le diabète - a tendance à faire des malades concernés des experts de leur propre prise en charge et si les diabétiques qui doivent s'injecter eux-mêmes quotidiennement leur dose d'insuline sont confrontés à l'usage systématique d'outils de mesure, c'est le patient qui, avec le développement de dispositifs connectés, est une nouvelle fois équipé et non pas le médecin (en tout cas, pas aussi directement). En l'occurrence, le rôle du médecin semble avoir tendance à s'effacer quelque peu, à devenir un peu plus secondaire, au profit de l'acteur non humain. Celui-ci - i.e. le dispositif de télédiabétologie et, plus fondamentalement, l'algorithme qui permet de calculer finement la dose d'insuline à s'injecter - tend à occuper une place plus centrale que dans les dispositifs antérieurs. Véritable " technologie réflexive" (Licoppe et al., 2013), il permet aux usagers d'élaborer un autre rapport à soi, plus resserré, plus calculé et plus instantané, et, de façon paradoxale - dans la mesure où les patients paraissent plus dépendants à l'égard de l'outil de calcul -, de gagner en autonomie dans le cadre de leur prise en charge quotidienne. Une analyse du fonctionnement et des usages des dispositifs de télémédecine et de télésanté doit ainsi prêter attention au degré de prééminence des acteurs non humains et à la façon dont ils contribuent à la reconfiguration de la prise en charge médicale (Oudshoorn, 2011).

13 Nous faisons référence ici au dispositif Diabéo mis en place par Sanofi en mars 2013 dans le cadre de la phase expérimentale de l'enquête Télésage. 
Nous trouvons, au-delà de la $m H_{\text {Health }}{ }^{14}$, d'autres expressions du phénomène d'endogénéisation : de nombreux sites Internet ont vu le jour, qui proposent des téléconsultations en santé mentale - par un psychologue et plus rarement un psychiatre. On voit ainsi apparaître aujourd'hui un nouveau marché des soins en ligne, avec des sites comme Tonpsy, Eutelmed, Goodpsy ou Doctair. Ces sites s'adressent en priorité à des patients de langue française qui peuvent résider en France ou à l'étranger. Leur fonctionnement repose sur une plateforme permettant, via Internet, de réaliser des téléconsultations en psychiatrie et en psychologie - suivant des modalités diverses selon que le professionnel est un médecin-psychiatre ou un psychologue clinicien. Si certains de ces sites offrent des relations visiophoniques sécurisées avec des professionnels qualifiés, ils empruntent tous leurs fonctionnalités aux sites Internet existants : l'inscription, le choix du thérapeute et la prise de rendez-vous se font en ligne, la relation thérapeutique prend la forme d'une relation visiophonique classique et le règlement s'effectue par un système sécurisé de paiement par carte bancaire en ligne-comme Paypal. $\mathrm{Si}$, du point de vue des pratiques professionnelles et interactionnelles, de nombreuses transformations sont observables, sur le plan technique, ces sites s'apparentent très largement aux sites traditionnels.

\section{Les effets du phénomène d'endogénéisation des dispositifs sur les usages}

Quelles relations existe-t-il entre le processus d'endogénéisation des dispositifs de la télémédecine et de la télésanté et les usages qui en sont faits ? Autrement dit, en quoi ce phénomène peut-il contribuer à configurer les usages des différents utilisateurs, qu'ils soient professionnels ou patients, et partant, à transformer la prise en charge elle-même et la façon dont les différents protagonistes définissent leur rôle (ou leur identité) dans ce cadre ? Un des enjeux est de saisir dans quelle mesure les standards adoptés constituent des contraintes sur le plan des usages et, inversement, en quoi ils demeurent éventuellement malléables, adaptables ou contournables, octroyant ainsi des marges de

14 La mHealth ou mSanté désigne l'ensemble des usages, dans le domaine de la santé, du téléphone portable et des technologies sans fil. 
manœuvre aux utilisateurs. Tout d'abord, rappelons que les dispositifs observés sont relativement instables. Pour une part d'entre eux, ils n'ont pas encore atteint le stade d'irréversibilité propre aux infrastructures - il semble même qu'ils ne l'atteindront jamais. Les acteurs peuvent ainsi façonner certaines de leurs caractéristiques principales. Et, comme nous allons le voir, même une fois que les dispositifs sont stabilisés - i.e. généralement après la phase expérimentale des projets et le passage en phase dite de routine -, leurs usages témoignent des marges d'action et des possibilités d'appropriation multiples dont bénéficient les usagers.

Parmi les effets du phénomène d'endogénéisation sur les usages, nous pouvons évoquer tout d'abord le fait que l'adoption des standards existants dans le cadre des nouveaux dispositifs de télémédecine et de télésanté semble faciliter pour une part les utilisations qui en sont faites. Ainsi, les patients français expatriés consultant un psychiatre ou un psychologue via le dispositif Télé-med sont de grands utilisateurs de logiciels visiophoniques comme Skype, ce qui contribue à rendre leurs échanges avec le thérapeute et leur élaboration (au sens psychologique du terme) plus aisés, les patients ayant l'habitude de verbaliser et de s'exprimer via ce type de dispositif de communication. Lorsque le site Internet ne fonctionne pas correctement, leur aisance dans les usages permet aussi de procéder à des remédiations techniques consistant à utiliser un logiciel de visiophonie classique. Dans certains cas, leur familiarité avec Skype les conduit à demander au thérapeute d'employer ce logiciel de façon systématique en invoquant sa souplesse d'utilisation. Lorsqu'une telle habitude est prise, le site Internet n'est alors plus utilisé comme artefact interactionnel et ses usages se limitent à ses fonctions de gestion, c'est-à-dire de programmation des séances et de paiement par carte bancaire, ce qui constitue un détournement des usages (Akrich, 1998), la fonction première du dispositif, qui est d'offrir une relation visiophonique sécurisée par Internet, ayant été abandonnée.

Les effets du phénomène d'endogénéisation sur les usages s'observent également dans l'adoption de pratiques caractérisant habituellement le e-commerce. Nous pouvons prendre l'exemple des professionnels de la relation psychothérapeutique, qui proposent leurs services au sein d'un marché des soins en ligne s'accompagnant de formes de concurrence inédites. Pour nombre de praticiens, la participation à ces dernières représente au premier abord un exercice souvent difficile, dans la mesure 
où elle suppose l'adoption d'une démarche commerciale jusqu'alors étrangère à leur pratique professionnelle. Auparavant, les modes de captation de la patientèle se fondaient sur des logiques de réputation, d'anciens patients ou des personnes de leur entourage proche, ou bien encore des professionnels de santé contribuaient à établir l'image de tel ou tel thérapeute concernant son efficacité, ses domaines de spécialité, voire d'autres caractéristiques comme sa disponibilité - pour obtenir un premier rendez-vous -, sa ponctualité, sa façon d'interagir (direct, doux, convivial, etc.). Dans le cadre de la pratique traditionnelle, le sentiment de concurrence vis-à-vis des confrères existe bel et bien, les psychothérapeutes sachant pertinemment qu'ils sont en concurrence avec certains de leurs confrères au sein d'une aire géographique plus ou moins clairement délimitée. Mais ce sentiment est plus diffus, dans la mesure où leur profil n'est pas directement mis en regard (et en concurrence) avec celui de tout un ensemble de confrères apparaissant au sein d'une liste figurant sur Internet. Désormais, les thérapeutes désireux de développer une activité professionnelle de téléconsultation doivent faire apparaître, de façon plus ou moins approfondie, dans une fiche de présentation mise en ligne sur le site du dispositif de téléconsultation, leur cursus, leurs domaines de spécialité, leur positionnement dans le champ psychothérapeutique - i.e. leur appartenance à un courant. Ils doivent aussi trouver des petites phrases accrocheuses et présenter une photo-portrait où ils sont à leur avantage afin de capter leurs futurs clients. Certains télépraticiens ironisent ainsi à propos de leur fiche de présentation en la comparant à celles qui apparaissent sur les sites Internet de rencontre. Dans le même ordre d'idées, ils insistent sur la part de banalisation, de "dé-spécification " de la relation thérapeutique qui, dans le cadre des téléconsultations, emprunte les mêmes voies de communication que les relations amicales, familiales, professionnelles ou commerciales. Selon eux, l'identité des dispositifs sur le plan technique conduit, symboliquement, à assimiler les relations thérapeutiques par téléconsultation aux échanges visiophoniques classiques et à les tenir éloignées de la relation psychothérapeutique traditionnelle qui se tient dans l'alcôve du cabinet, autrement dit, au sein d'un lieu spécifique constituant une composante essentielle du cadre thérapeutique - i.e. un espace-temps marqué par sa singularité, sa régularité et son caractère contenant au sens psychologique du terme. 
Une autre illustration - et non des moindres - de l'endogénéisation des usages et des dispositifs de la télémédecine dans les standards des infrastructures existantes est fournie par tout un ensemble de pratiques que nous pouvons désigner comme relevant de la " télémédecine informelle ". Il s'agit d'usages prenant corps en dehors ou à la frontière du cadre légal, ne donnant pas lieu officiellement à un acte de télémédecine et reposant sur les réseaux professionnels de relations, de coopération et de confiance des protagonistes. La télémédecine informelle s'apparente généralement à la télé-expertise ou à la pratique de la téléconsultation. Les canaux et dispositifs de communication employés sont similaires, sinon identiques à ceux utilisés dans le cadre de la télémédecine " officielle ", qui, elle, donne lieu à la production d'actes de télémédecine, apparaît dans les projets d'établissement, fait l'objet d'accords entre partenaires, nécessite d'obtenir l'aval des instances officielles comme l'ARS et, le cas échéant, le Conseil national de l'Ordre des médecins (CNOM). La télémédecine informelle, qui n’a pas donc d'existence officielle, constitue une forme d'endogénéisation complète d'un point de vue technologique - mais incomplète du point de vue juridique et institutionnel. Le plus souvent, la télémédecine informelle mobilise exactement les mêmes standards technologiques que la télémédecine officielle. Elle ne nécessite pas l'acquisition d'un nouvel objet technique ni une nouvelle technologie de l'information et de la communication, mais repose seulement sur l'utilisation des outils et des technologies existants et disponibles.

La télé-expertise informelle peut être observée notamment en radiologie ou en dermatologie (par l'envoi de clichés à un confrère via un e-mail, un MMS ou l'envoi de gros fichiers sur Internet). Elle peut aussi avoir lieu en cardiologie (avec l'envoi d'électrocardiogrammes à un confrère). Utilisée dans un souci d'efficacité, pour réduire l'incertitude du médecin face à un cas clinique, elle se caractérise par le recours à l'avis d'un confrère de son réseau de relations. En dermatologie, la télé-expertise informelle repose très souvent sur l'usage du smartphone qui permet de réaliser et d'envoyer très rapidement à un confrère des clichés d'une lésion cutanée à propos de laquelle l'expéditeur souhaiterait recueillir - de façon officeuse - son avis. De telles pratiques sont développées par un grand nombre de dermatologues depuis de nombreuses années - dermatologues, qui, dans la tradition hospitalière, avaient pour 
habitude d'utiliser des clichés photographiques pour étudier l'évolution des lésions cutanées. Le procédé de la télé-expertise en dermatologie est aussi jugé utile dans la mesure où il permet d'obtenir l'avis d'un confrère très rapidement.

En psychiatrie, la téléconsultation est utilisée de façon informelle en diverses occasions. Il arrive qu'un patient ne puisse pas se déplacer au cabinet du praticien, en raison d'un problème physique conduisant à une immobilisation temporaire - e.g. une jambe cassée - ou en raison de la manifestation d'un trouble psychique spécifique comme l'agoraphobie. D'un commun accord avec le patient, le praticien peut décider, en pareils cas, de réaliser une ou plusieurs séances par le biais d'un dispositif visiophonique, tel Skype. Cette pratique s'inscrit en dehors du cadre légal, dans la mesure où les séances ne relèvent pas officiellement de la télémédecine : elles sont comptabilisées comme des séances face-à-face et ne bénéficient pas des autorisations de l'Agence régionale de santé.

En télépsychiatrie cette fois, il arrive que les acteurs doivent procéder à des remédiations techniques en cas de défaillance de la connexion Internet ou de problèmes de fonctionnement du site utilisé habituellement pour réaliser les téléconsultations. Ainsi, les consultations à distance s'effectuent en mobilisant un autre moyen de communication, à commencer par les dispositifs visiophoniques classiques, qui offrent une connexion moins sécurisée, mais qui permettent à la téléconsultation d'avoir lieu malgré tout. Lorsque la connexion demeure médiocre en dépit du changement de dispositif, les protagonistes coupent l'image pour garantir un niveau de qualité sonore jugé satisfaisant, voire décident de terminer la séance par un autre moyen. Certaines téléconsultations ont ainsi lieu par téléphone (portable ou fixe), les utilisateurs passant d'une infrastructure (Internet) à l'autre (réseau téléphonique) ; parfois la messagerie électronique est utilisée, mais plutôt pour clore l'échange. Toujours dans le cadre de la télépsychiatrie, des formes de télé-expertise informelle viennent parfois se greffer aux téléconsultations " officielles". Il arrive en effet que le médecin (le plus souvent un généraliste) qui accueille le patient dans la salle de téléconsultation s'entretienne informellement avec le psychiatre à l'issue de la séance. Ce type d'échange non programmé s'établit dans le cadre même des séances de téléconsultation officielle 
mais pose des problèmes de catégorisation face aux standards adoptés par le législateur, dans la mesure où il ne s'intègre dans aucune des catégories officielles concernant les actes de télémédecine. Une telle pratique s'apparente à la télé-expertise (synchrone), mais ne s'y réduit pas, dans la mesure où les thèmes abordés ne sont pas liés seulement au cas d'un patient donné mais touchent au fonctionnement et aux usages individuels et collectifs du dispositif lui-même. L'expression " télé-staff» ou "session technique » est parfois employée par les utilisateurs pour désigner une pratique courante et informelle consistant, avant ou après la téléconsultation avec un ou plusieurs patients, en un échange entre les professionnels de santé participants (médecins et paramédicaux) situés de part et d'autre du dispositif.

\section{Conclusion}

La notion d'infrastructure présente des vertus heuristiques à plus d'un titre. Tout d'abord, elle permet d'articuler les échelles d'analyse micro et macro - et donc de ne pas se focaliser sur le seul niveau microsociologique, sans toutefois négliger cette entrée analytique, la question des usages et des utilisateurs constituant une des composantes de la perspective en termes d'infrastructures dont nous nous sommes inspiré. Nous avons ainsi été amené à prendre en considération l'infrastructure institutionnelle sur la base de laquelle les projets de télémédecine se développent aujourd'hui, tout en montrant que ce développement de la télémédecine officielle s'accompagne de celui de la télémédecine informelle. Nos observations empiriques nous conduisent à penser que ces deux formes de télémédecine se renforcent mutuellement, le fait de pratiquer la télémédecine informelle facilitant ou prédisposant à l'engagement dans des projets et des dispositifs de télémédecine officiels - et inversement. Nous faisons l'hypothèse que ces deux modes d'existence de la télémédecine auront vocation à coexister à l'avenir, comme semblent déjà le confirmer les usages de nombre de praticiens. Vraisemblablement, la pratique de la télémédecine informelle se perpétuera en raison de sa grande souplesse d'utilisation.

D’un point de vue général, le recours à la notion d'infrastructure permet de mieux prendre en considération le contexte sociotechnique, juridique et institutionnel dans lequel se développent la télémédecine 
et la télésanté. Tout l'enjeu des recherches empiriques est de déterminer en chaque cas jusqu'à quel point leurs dispositifs diffèrent de ceux des infrastructures existantes, mais aussi sont façonnés par elles. Le processus d'endogénéisation des dispositifs et des pratiques de la télémédecine et de la télésanté dans les standards des infrastructures existantes ne peut ainsi être perçu strictement sous l'angle de la contrainte, les pratiques des professionnels témoignant de l'existence d'importantes marges d'action dont bénéficient les usagers, comme l'illustrent notamment les situations de remédiation technique en cas de défaillance d'une infrastructure ou d'un dispositif, ainsi que la pratique informelle de la télémédecine. Sur ce point, l'utilisation des technologies de l'information et de la communication dans le monde de la médecine et de la santé pose, à nouveaux frais, la question de la dépendance des acteurs sociaux à l'égard des dispositifs et des infrastructures qui les sous-tendent. Si les infrastructures ont tendance à se faire oublier lorsqu' elles fonctionnent de façon fluide, nous pouvons a priori nous interroger sur l'importance, en termes de santé publique, de leurs éventuelles défaillances. Au-delà des infrastructures elles-mêmes, et à plus petite échelle, la question se pose également à l'égard des possibles dysfonctionnements des dispositifs locaux et des objets techniques qui les composent. Nous pensons ici aux objets connectés personnels comme la tablette ou le smartphone qui, vraisemblablement, seront amenés dans un avenir proche à jouer un rôle de plus en plus important dans la prise en charge quotidienne de diverses catégories de patients ou d'usagers des dispositifs de télémédecine et de télésanté, dans un contexte marqué par l'avènement des usages des outils numériques et le développement de la médecine ambulatoire, ainsi que par le vieillissement de la population et l'augmentation des maladies chroniques qui l'accompagne.

\section{Références}

Akrich M. (1998), "Les utilisateurs, acteurs de l'innovation ", Éducation permanente, $\mathrm{n}^{\circ} 134$, p. 79-89.

Bowker G. C., Star S. L. (1999), Sorting Things Out. Classification and its consequences, Cambridge (Mass.), The MIT Press.

Bonu B. (2007), "Connexion continue et interaction ouverte en réunion visiophonique ", Réseaux, n 144, p. 25-57. 
Dodier N. (1993), L'expertise médicale. Essai de sociologie sur l'exercice du jugement, Paris, Métailié, coll. «Leçons de choses ».

Edwards P. N. (2003), «Infrastructure and Modernity: Force, Time, and Social Organization in the History of Sociotechnical Systems ", dans Misa T. J., Brey P., Feenberg A. (éds), The History of Sociotechnical Systems: Modernity and Technology, Cambridge (Mass.), The MIT Press, p. 185225.

Edwards P. N., Bowker G. C., Jackson S. J., Williams R. (2009), "Introduction: An Agenda for Infrastructure Studies ", Journal of the Association for Information Systems, special issue, vol. 10, n 5, p. 364-374.

Hughes E. C. (1996), Le regard sociologique. Essais choisis, Paris, Éditions de l'EHESS.

Hughes T. P. (1999), "The Evolution of Large Technological Systems ", dans Biagioli M. (éd.), The Science Studies Reader, New York-Londres, Routledge, p. 202-223.

Licoppe C., Draetta L., Delanoë A. (2013), “ Des "smart grids" au "quantified self". Technologies réflexives et gouvernement par les traces, une étude de cas sur la consommation électrique en milieu domestique ", Intellectica, vol. 1, $\mathrm{n}^{\circ}$ 59, p. 267-290.

Mathieu-Fritz A. (2015), "Le travail réel, part oubliée des politiques publiques de santé ", Dsih, avril.

Mathieu-Fritz A., Esterle L. (2013), "Les médecins et le dossier santé informatisé communiquant. Analyse d'une expérimentation du dossier médical personnel (DMP) ", Réseaux, n 178-179, p. 209-242.

Mathieu-Fritz A., Esterle L. (2013a), "Les transformations des pratiques professionnelles lors des téléconsultations médicales. Coopération interprofessionnelle et délégation des tâches ", Revue française de sociologie, vol. 54, n², p. 303-329

Mathieu-Fritz A., Esterle L. (2013b), «Teleconsultation in geriatrics: impact on professional practice ", International Journal of Medical Informatics, $\mathrm{n}^{\circ}$ 82, p. 684-695.

Mathieu-Fritz A., Esterle L., Espinoza P. (2011), «L'impact des consultations à distance sur les pratiques médicales : vers un nouveau métier de médecin ? ", Revue française des affaires sociales, n 2-3, p. 105-122. 
Oudshoorn N. (2011), Telecare Technologies and the Transformation of Healthcare, Londres, Palgrave Macmillan.

Relieu M. (2007), "La téléprésence, ou l'autre visiophonie », Réseaux, $\mathrm{n}^{\circ} 144$, p. 183-223.

Star S. L. (2010), "Ceci n'est pas un objet-frontière ! Réflexions sur l'origine d'un concept ", Revue d'anthropologie des connaissances, vol. 4, $\mathrm{n}^{\circ} 1$, p. 18-35.

Strauss A. L. (1992), La trame de la négociation. Sociologie qualitative et interactionniste, Paris, L'Harmattan. 
INFRASTRUCTURES ET POLITIQUE 



\title{
Les communs et les infrastructures des villes
}

\author{
Gilles Jeannot
}

Les communs, ces biens matériels partagés dans une communauté apparaissent comme un point d'appui et une source de réflexion stimulante pour un réinvestissement du politique par l'aval. Dans les contextes urbains, cependant, d'autres biens matériels s'imposent pour toute réflexion concrète sur la constitution du politique : les infrastructures.

\section{Communs urbains et oubli des infrastructures}

La notion de communs trouve plutôt son ancrage dans des contextes agricoles ou de pêche (pâtures communes, forêts, étangs, mers...) (Bourjol, 1989 ; Ostrom, 2015), mais elle a également été mobilisée dans des contextes urbains. Les applications les plus immédiates de la notion de communs dans les villes se trouvent dans des réappropriations collectives à l'échelle du microquartier comme dans le cas de jardins partagés, d'habitat participatif, de boîtes d'échange entre voisins comme cela a été illustré dans le réseau "villes en (biens) communs ». Mais la notion est aussi investie à l'échelle de l'ensemble de la cité. Deux cas de figure contrastés illustrent cela : les mouvements pour la déprivatisation de services urbains d'une part et les initiatives pour une cartographie alternative ; le premier comme réappropriation de communs traditionnels et les seconds pour les communs de la connaissance.

Aqua bene commune, eau bien commun, c'est autour de ce slogan que s'est coalisé un mouvement italien contre la privatisation de l'eau. En 2011, en Italie, une loi de Berlusconi rendant contraignante la délégation de la gestion de l'eau à des prestataires privés a suscité l'émergence d'un forum original qui rassemblait militants, nouveaux venus à la politique 
et associations catholiques. Ils ont obtenu un référendum d'initiative populaire qui mobilisera 27 millions d'électeurs. La loi sera abrogée et dans la foulée des expériences innovantes de réappropriation de la distribution de l'eau ont été engagées. En Allemagne, un référendum d'initiative citoyenne à Berlin, en 2011, contre des clauses secrètes incluses dans le contrat de concession de l'eau a conduit indirectement à un retrait de l'opérateur privé. À Hambourg, c'est aussi un mouvement citoyen qui a imposé la remunicipalisation du service de distribution d'électricité. En France, Paris a repris en régie en 2010 la distribution de son eau confiée à des opérateurs privés en mettant en avant l'intérêt d'une gestion intégrée du bien commun de l'eau de la production au retraitement. Plusieurs villes françaises suivent cette voie, ce qui conforte le pôle public de l'eau. Dans ces quelques expériences européennes, les déprivatisations ont été d'abord pensées par leurs acteurs comme réappropriation de communs. Les préoccupations écologiques et la recherche de préservation de biens publics par des formes alternatives de production ou la quête d'une maîtrise démocratique renforcée expliquent tout autant ces mouvements que les préoccupations économiques (Jeannot et Coutard, 2015 ; Blanchet et Herzberg, 2017).

Le courant des creative commons a également un volet territorial et urbain avec le mouvement de l'information géographique volontaire. L'ensemble des pratiques repose sur la possibilité offerte d'une géolocalisation des smartphones par GPS. Des volontaires peuvent enrichir une base donnée sur leur territoire, faire connaître des aménités urbaines ou relever des dysfonctionnements en les localisant avec les fonds de cartes proposés par Google. Ces pratiques ont pu être tout particulièrement mobilisées dans des pays des Suds dans lesquels l'offre publique en matière de services urbains est défaillante et permettre une appropriation par la population de ces questions. C'est ainsi qu'au Liban les citoyens se sont mobilisés pour faire face à une crise sans précédent dans le ramassage des déchets en localisant les décharges abusives, ou qu'en Afrique des cartes des services sont établies de manière collaborative face à l'absence d'offre publique. De manière plus radicale, le mouvement openstreetmap créé au Royaume-Uni en 2004 ambitionne de créer des fonds de cartes en open source. Les militants parcourent alors les itinéraires de transports en commun en mémorisant à un rythme 
régulier leur position, permettant de créer de manière autonome un fond de carte libre de droits dans la lignée directe du courant pour le logiciel libre.

La quête d'une refondation de ce qui est public à partir des communs, cependant, laisse dans l'ombre une notion tout aussi essentielle : celle d'infrastructure. La référence aux communs est bien légitime pour ce qui concerne l'eau en ce que celle-ci est puisée et retournera à la nappe qui est bien un " common ». Elle peut aussi se justifier en partie pour ce qui concerne l'électricité en ce que la production d'énergie interagit avec divers biens communs de la planète. Mais cette référence n'épuise pas la spécificité de ces biens et services. Pas de robinet sans canalisations, stations de pompage et d'épuration, pas de courant dans les ampoules sans câbles, pas de service sans infrastructures. La présence des infrastructures dans le cas des cartographies est moins visible, elle n'en est pas moins essentielle. Les données utilisées circulent dans des réseaux Internet puis sont stockées dans des fermes d'ordinateurs qui sont des infrastructures bien matérielles. Les données GPS ne « tombent pas du ciel ", elles sont offertes par l'infrastructure lourde que représente le maillage de satellites mis à disposition par l'armée américaine.

Mettre en avant les infrastructures plutôt que les communs n'est toutefois pas laisser de côté la question du public pour des dimensions plus pratiques et fonctionnelles. En effet, un lien tout aussi essentiel s'est tissé au cours de l'histoire entre les infrastructures et ce qui est public. Pour aller plus loin dans la réflexion, un parallèle peut être établi entre la notion de communs et celle d'infrastructure dans ce qu'elles participent à la constitution du public dans la perspective pragmatique d'un John Dewey (2010).

\section{L'infrastructure et les communs, deux fondements parallèles de ce qui est public}

Dans les deux cas, le point de départ est une réalité matérielle. C'est autour de pâtures partagées, d'étangs ouverts à la pêche, ou de forêts, que s'est construite la réflexion sur les communs. De la même manière, les infrastructures sont des tuyaux, des voiries des rails, des bâtiments et des machines. La réflexion contemporaine sur les communs tend à 
minimiser l'importance de cette matérialité, Dardot et Laval (2014) en particulier se sont efforcés de construire une théorie du commun qui s'affranchisse de cette matérialité et d'un certain économicisme. Et on peut tout à fait justement remarquer que cette matérialité, hors de tout contexte institutionnel, ne produit pas de fait public à elle seule. Les terres anglaises qui étaient des communs ont cessé de l'être lorsqu'elles ont été encloses. Ou, comme le remarque Allan Greer (2015), les terres partagées ont pu dans des contextes coloniaux s'avérer une ressource pour les plus forts. Néanmoins, dans le cas des communs, la spécificité de l'approche par les communs s'évanouirait si on abandonnait toute référence à cette matérialité et que l'on en revenait aux questions plus classiques du lien social ou de l'égalité. Il en va de même pour les infrastructures.

Dans les deux cas, cette réalité matérielle est considérée par les économistes comme singulière, et d'une certaine manière réfractaire au marché non organisé. On connaît la distinction établie par les économistes entre les biens publics et les biens privés et les catégories différentes de biens publics dégagées. La notion de " monopole naturel » est moins connue, elle recouvre deux arguments. John Suart Mill notait en 1848 : «Il est évident qu'on aurait pu économiser beaucoup de travail si Londres était approvisionnée par une seule compagnie d'eau ou de gaz plutôt que par la pluralité existante. " Le monopole apparaît dans ces situations comme l'optimal économique. C'est une perte d'efficacité que d'installer deux réseaux en parallèle alors qu'un seul peut offrir le service à tout le monde. On ne connaît pas de cas où deux compagnies d'eau installeraient deux réseaux d'eau parallèles qui aboutiraient sur deux robinets à chaque domicile et de même, à de rares exceptions près (dont les deux compagnies qui ont desservi à l'origine en concurrence Versailles Rive Gauche et Versailles chantier), une seule infrastructure ferroviaire a été établie entre deux villes, même quand le marché était privé. Le partage du marché s'est fait par grands monopoles territoriaux dont les gares parisiennes étaient les têtes de pont. Le second argument que l'on peut rapporter à Léon Walras n'est plus lié à l'efficacité, mais à la dynamique économique. Lorsqu'il y a de coûteuses infrastructures, le premier arrivé, dont une partie du capital est amorti, se trouve avantagé par rapport à ses concurrents, il peut les conduire à la faillite, les racheter, s'agrandir et renforcer encore son avantage. On observe alors selon 
les conditions initiales l'émergence d'un monopole ou d'un duopole. Cela explique qu'au-delà du monopole local sur un microterritoire, ce sont les mêmes compagnies qui pourront progressivement rassembler ces réseaux locaux. Cette explication vaut largement pour le secteur électrique et pour les entreprises du secteur de l'eau.

Dans les deux cas, la notion peut s'appliquer avec quelques ajustements aux dimensions immatérielles plus contemporaines de la connaissance. Le renouveau de la notion de commun est indissociable de son application aux creative commons (logiciels libre, wikis) (Coriat, 2015). L'idée, plus étonnante, $d$ ' « infrastructure de la connaissance » a été introduite en particulier par Paul Edwards et al. (2012). Le point de départ est la remarque que, derrière tous les développements de l'Internet qui nous paraissent largement immatériels, se trouvent de manière non métaphorique et toute matérielle des infrastructures lourdes. Ce sont les câbles sous-marins qui transportent les flux de données entre continents, les fermes d'ordinateurs qui permettent de les stocker. Ce sont des systèmes d'ordinateurs interconnectés qui permettent des calculs scientifiques entre universités dans le projet Terragrid. Les infrastructures de la connaissance et les infrastructures traditionnelles ne cessent en outre aujourd'hui également de s'entremêler dans le champ urbain. La plupart des infrastructures traditionnelles sont aujourd'hui recouvertes d'une peau digitale, de la multiplication de capteurs et de bases de données, ce que l'on appelle smart city. Les services de transports deviennent indissociables de masses de données collectées centralement par les pouvoirs publics qui orientent le trafic avec des panneaux à messages variables ou par des applications privées comme Waze, qui orientent les conducteurs en redistribuant les informations apportées par les membres.

Dans les deux cas, cette spécificité joue un rôle dans les rapports de classe. On connaît, depuis Karl Polanyi (1983), la fable sur les " enclosures " (la pose de clôtures autour des terrains communaux partagés) comme captation par les riches des communaux appropriés par les pauvres qui se trouveront rejetés sur les routes. On sait aussi comment cela a été généralisé autour des nouvelles enclosures liées aux communs de la connaissance et théorisé comme une actualisation du slogan de Proudhon "la propriété c'est le vol " (comme le pointent malicieusement Dardot et Laval, 2014). Mais on peut aussi à la suite 
d'Abram de Swann (1995) décrire le développement des réseaux d'infrastructure comme un vol des riches par les pauvres. Les premiers réseaux d'eau, d'électricité, de téléphone ont été établis pour des hôtels de luxe ou par un groupe réduit de bourgeois urbains pour leur propre usage et étendus de proche en proche à d'autres familles capables de payer le service. Cependant, l'universalisation du service et son extension aux banlieues populaires puis au milieu rural ne peuvent se faire par la simple diffusion de proche en proche et supposent une réappropriation du réseau initial par la collectivité publique au profit de tous : riches et pauvres, urbains et ruraux. Ce ne sont bien sûr dans les deux cas que des fables simplificatrices. Le récit sur les enclosures vaut plus pour le cas britannique que pour le cas français pour lequel, comme le rappelle Bourjol (1989), c'est une appropriation publique des communs plus progressive qui a été accomplie. De même, le récit d'Abram de Swann correspond à la réalité historique pour le cas de l'eau (avec en sus un enjeu d'hygiène), pour l'électricité et le téléphone des origines, l'intervention publique dans d'autres réseaux comme la route ou la poste sera plutôt motivée par des visées régaliennes. Cependant, quelle que soit la fiabilité historique de ces fables, elles attirent notre attention sur les rapports sociaux engagés dans le déploiement de ces objets singuliers.

Dans les deux cas, ces "échecs du marché " ont conduit à des interventions publiques spécifiques : la création d'institutions particulières de propriété et de gestion partagée pour les biens communs tels que formalisés par Elinor Ostrom (2015), les municipalisations ou nationalisations pour les infrastructures de réseau. Les nationalisations sont ainsi à la fois les conséquences d'évolutions économiques et de choix politiques. L'histoire de la nationalisation de l'électricité et des chemins de fer au sortir de la Seconde Guerre mondiale illustre bien cette double dimension. La nationalisation comme rationalisation de l'offre d'infrastructure et la péréquation tarifaire promue par les ingénieurs d'une part et la nationalisation comme projet politique portée par le Conseil national de la résistance. Ces deux perspectives se différencient cependant fortement par les conséquences sur les modalités de l'intervention publique. Les modalités de gestion des communs associent diverses parties prenantes pouvant inclure ou non les collectivités publiques; la prise en charge des monopoles naturels s'est construite pour l'essentiel autour des collectivités publiques, municipalités ou 
État, et a conduit de fait à une mise à distance des usagers. Ces derniers acceptent à la fois d'être exclus de la décision (comme cela avait été envisagé dans des projets de cogestion de l'après-guerre) et de ne pas réclamer de payer le coût de leur propre consommation.

\section{Composer communs et infrastructures pour construire le public}

Le succès de la notion de commun doit se comprendre à partir de sa dimension concrète : partir d'objets singuliers, des relations sociales qui se nouent autour, inventer des formes de coordination démocratique pour la gestion de ces objets et en retour revivifier le lien politique. Poussant l'avantage, les promoteurs des biens communs sont tentés d'opposer ce caractère concret à une représentation plus abstraite de l'État. Pour le cas de l'eau, c'est ainsi en s'appuyant sur la notion de communs que Benjamin Coriat (2015) met, par exemple, en avant l'expérience de cogestion de l'eau à Naples contre la remunicipalisation portée par les élus à Paris.

Cependant, les infrastructures ne sont pas moins une dimension concrètedu publicquenelesontles communs. C'estcommepourlagestion partagée d'une pêcherie, un condensé historique de rapports sociaux qui se trouvent dans notre branchement électrique, même si la gestion en est publique. Cet oubli de l'épaisseur publique des infrastructures est aussi un produit historique, celui de leur invisibilisation. Alors que les communs sont par nature l'objet de conflits d'usages et se rappellent de ce fait à tout moment à l'attention, les infrastructures, lorsqu'elles sont installées et fonctionnent sans heurts, disparaissent même quand elles ne sont pas enterrées, comme les réseaux d'eau, d'électricité ou de téléphone. Cette invisibilisation des rapports sociaux a d'ailleurs été une force du déploiement de ces infrastructures, passer de la fontaine commune au robinet à domicile est bien évidemment plus pratique, mais c'est également le moyen d'éviter tous les conflits d'usage autour de ce point d'eau. Mais, cette invisibilisation devient aujourd'hui un handicap lorsqu'il s'agit de trouver un soutien dans la population à la défense de leur gestion publique, celle-ci ne semblant plus portée que par les syndicats et agents publics soupçonnés de défendre leurs intérêts plus que les biens publics. 
C'est ainsi seulement par une composition de ces deux notions que l'on peut contribuer à enrichir une approche matérielle permettant de réancrer le lien au public. Communs et infrastructure comme catégories d'analyse des réalités urbaines offrent en effet souvent moins une partition des cas que des angles différents pour appréhender des objets à facettes multiples. On peut rappeler au passage que le terme "usager » qui était utilisé au Moyen Âge pour désigner les " commoners " l'a été à partir du début du siècle pour désigner les bénéficiaires des nouvelles infrastructures publiques.

C'est un reproche justifié à la prise en charge par l'État ou les municipalités de ces services d'avoir fait oublier la dimension de biens communs qui se trouve attachée à ces services qui arrivent dans nos maisons et de déresponsabiliser l'usager. Des formes de renouvellement de l'attachement des citoyens au service public et d'ouverture de la participation à la décision et à la gestion peuvent permettre de contrecarrer cette tendance. Les actions concernant la propreté des rues ou les déchets, qui mettent en évidence la complémentarité d'une responsabilité des habitants (ne pas jeter de papiers gras, trier ses déchets) et l'offre publique des services de la voirie, illustrent cela. Cela peut se traduire aussi par les cartographies « fix my street " qui associent gestion publique des infrastructures et responsabilité des citoyens qui signalent des trous dans les trottoirs ou par une réactivation de la démocratie participative autour de ces questions d'infrastructure qui apparaissent souvent techniques et rébarbatives comme dans le débat citoyen ouvert à Paris sur le maintien du réseau d'eau parallèle permettant de nettoyer les rues.

Mais en retour l'isolement de la notion de commun soit limite très fortement ce qui est public, comme dans le cas des jardins partagés, soit conduit à une réappropriation très partielle par les " commoners " des biens publics considérés. L'exemple le plus patent, de ce point de vue, concerne les alternatives à Google map. Open street map défie Google avec l'aimable service de l'armée américaine. Ne pas s'interroger en parallèle de la critique de Google sur les infrastructures alternatives au GPS comme le projet européen Galileo (un rare nouveau service public promu par l'Europe, même si le financement se fait par un partenariat public privé) conduit à restreindre fortement l'ampleur de la réappropriation collective. Cet exemple peut être étendu à 
l'ensemble des initiatives urbaines de créations de communs tant elles supposent en arrière-plan le bon fonctionnement des infrastructures : approvisionnement en eau et retraitement, téléphone, électricité, traitement des déchets. Au-delà du " ni marché ni État " qui fédère le mouvement pro-communs, la préservation d'une gestion publique de ces infrastructures apparaît complémentaire des initiatives communautaires de gestion partagée qu'elles supportent. À l'exemple emblématique pour les promoteurs des communs du mouvement des citoyens napolitains qui ont isolé une gestion collective de l'eau bien commun, on peut alors opposer le cas des services publics urbains allemands. Le mouvement pour les déprivatisations s'est en effet développé à l'articulation d'un mouvement citoyen orienté vers les communs et d'un réseau des entreprises publiques multisectorielles de service public (Stadtwerke) qui ont porté avec succès devant les instances européennes leurs revendications concernant la préservation face aux règles de la concurrence d'un modèle original d'entreprise publique de services urbains.

\section{Références}

Blanchet T., Herzberg C. (2017), «Étude sur la gouvernance et l'organisation des services d'eau potable retournés en gestion publique en France (20002016) ", Working Paper du LATTS, n 17-09, mai.

Bourjol M. (1989), Les biens communaux : voyage au centre de la propriété collective Paris, Librairie générale de droit et de jurisprudence.

Coriat B. (éd.) (2015), Le retour des communs : la crise de lidéologie propriétaire, Paris, Les Liens qui libèrent.

Dardot P., Laval C. (2014), Commun : essai sur la révolution au XXI siècle, Paris, La Découverte.

Dewey. (2010), Le public et ses problèmes, traduit de l'anglais et préfacé par J. Zasc, Tours, Farrago. Rééd. Paris, Gallimard, coll. « Folio ».

Edwards P. N., Jackson S. J., Chalmers M. K., Bowker G. C., Borgman C. L., Ribes D., Burton M., Calvert S. (2012), " Knowledge Infrastructures: Intellectual Frameworks and Research Challenges ", Report of a workshop sponsored by the National Science Foundation and the Sloan 
Foundation, University of Michigan School of Information, 25-28 May, http://pne.people.si.umich.edu/PDF/Edwards_etal_2013_Knowledge_ Infrastructures.pdf (consulté le 26 juin 2017).

Greer A. (2015), "Confusion sur les communs ", La Vie des idées, 31 mars.

Jeannot G., Coutard O. (2015), Revenir au service public ?, Paris, La Documentation française.

Ostrom E. (2015), Governing the commons : the evolution of institutions for collective action, Cambridge, Cambridge University Press.

Polanyi K. (1983), La grande transformation : aux origines politiques et économiques de notre temps, traduit de l'anglais par C. Malamoud et M. Angeno, Paris, Gallimard.

De Swaan A. (1995), Sous l'aile protectrice de l'État, Paris, Presses universitaires de France. 


\title{
Infrastructures numériques et production d'environnements personnalisés
}

\author{
Dominique Cardon
}

La dimension matérielle des infrastructures numériques a été récemment soulignée par une série de travaux qui est venue lester l'économie immatérielle d'un poids considérable d'appareillages de transmission, de routeurs, de câbles sous-marins et d'équipements dont le fonctionnement requiert une ingénierie complexe et de solides réseaux organisationnels. Le rôle des tuyaux de l'Internet (Blum, 2012), l'empreinte environnementale des fermes de serveurs (Maxwell, 2012), la recentralisation géographique du réseau (Beaude, 2014) ou la gouvernance des différentes couches de protocoles permettant au réseau des réseaux de fonctionner ont été réintroduits dans le débat scientifique (DeNardis, 2014). Cette exploration des formes équipées de l'infrastructure numérique nourrit des réflexions mettant en avant les dimensions géographiques, géologiques et écologiques des processus de numérisation (Bratton, 2015), les enjeux de classification et d'organisation technique des informations dans un univers où les nomenclatures catégorielles ont été bouleversées (Kitchin, 2014), le rôle systémique de phénomènes comme le spam dans l'architecture d'Internet (Burton, 2016) ou bien la généralisation de la forme média à des artefacts de plus en plus divers (Durham, 2015). Elle invite à enraciner les dimensions immatérielles des échanges entre utilisateurs dans les différentes strates matérielles qui les rendent possibles. Aussi, dans une très ambitieuse construction, Benjamin Bratton (2015) propose-t-il une cartographie de cet "empilement" (stack) en six " couches " qui façonne le nouvel assemblage numérique. Au-dessus de la terre qui lui fournit des ressources, le cloud installe l'armature des serveurs et des bases de données sur laquelle vient se poser la ville qui concentre les activités et les échanges à distance. Au-dessus de ces trois premières " couches ", Benjamin Bratton situe les adresses qui individualisent à la 
fois les humains et les objets connectés puis les interfaces qui structurent les interactions possibles entre les agents et leur environnement. Enfin, au-dessus de ces cinq strates empilées de l'infrastructure numérique s'active l'utilisateur. "Chacun de nos clics ", résume Yves Citton, " envoie [...] un courant électrique qui traverse l'empilement de haut en bas, avant de remonter pour faire surface auprès d'une série d'autres utilisateurs, qui enverront sans doute alors à leur tour une série d'autres signaux électriques - traversant et agitant à chaque fois, à la vitesse de la lumière, certaines Interfaces localisables à certaines Adresses, connectant certaines Villes au sein des vastes plateformes du Cloud, consommant toujours davantage de ressources de notre Terre au fur et à mesure de l'étirement, de l'accélération et de l'intensification des frissons qui y circulent "(Citton, 2016). Ces perspectives invitent à penser ensemble les incessantes articulations entre le hardware et le software, le code et la loi ${ }^{1}$. Elles attirent l'attention sur la complexification des interdépendances entre les enjeux environnementaux, la structuration matérielle des systèmes sociotechniques, la métrologie des standards techniques, le développement économique de puissantes plateformes de services, la régulation étatique et la diversification des usages. C'est dans une direction parallèle, mais légèrement différente que l'on voudrait proposer un cadre de réflexion sur l'" infrastructuralisation " grandissante des formes prises par le calcul algorithmique dans les mondes numériques. Comme y invite l'introduction de cet ouvrage, les dispositifs numériques associent les individus à l'infrastructure technique par l'intermédiaire de nouvelles techniques de calcul. Avec le développement de la calculabilité algorithmique (Rouvroy et Berns, 2010), les systèmes techniques (capteurs, interfaces d'information et de communication, bases de données, etc.) ont de plus en plus pour ambition non tellement de mesurer et d'organiser l'espace pour tous, mais de fabriquer un environnement calculé pour chacun qui se manifeste sous la forme de listes, de recommandations, de guidages géographiques ou de notifications. Bien qu'elle soit encore loin d'être réalisée en pratique, c'est cette ambition que l'on voudrait caractériser dans ce texte. Beaucoup des débats entourant les big data portent sur le volume et l'hétérogénéité des nouvelles données numériques, mais sans

Très influente, une première approche de la matérialité contraignante du code informatique avait été initiée par Lawrence Lessig (2006). 
doute l'aspect le plus original de cette promesse technologique réside$\mathrm{t}$-il dans la volonté d'instaurer en temps réel une boucle de rétroaction permettant de modifier l'environnement de l'utilisateur en fonction de ses comportements ${ }^{2}$.

Beaucoup de réflexions sur le développement de la calculabilité dans les mondes numériques opposent, d'une part, les entreprises qui construisent les grandes plateformes des services numériques et, derrière elles, les infrastructures sociotechniques leur permettant de collecter les données des utilisateurs et, d'autre part, les individus qui se trouvent soumis au pouvoir invisible et secret des calculs. Le spectre orwellien d'une surveillance totale, la domestication des internautes enfermés dans les rets des plateformes, le traçage intime et tatillon de la "vie elle-même " constituent autant de motifs constamment associés à la digitalisation accélérée de sphères toujours nouvelles de la vie sociale (Sadin, 2015). L'opposition frontale entre les individus et les infrastructures technicoéconomiques des grands acteurs du numérique conduit à interpréter le pouvoir de ces derniers en termes de rationalisation, de disciplinarisation ou de contrôle des subjectivités en empruntant le vocabulaire notionnel des approches critiques des technosciences. Même si elle n'est en rien infondée, cette perspective rate en grande partie sa cible en projetant sur les nouvelles formes de calcul des individus des résistances et des refus qui ont été construits en opposition à un état ancien de la domination par les chiffres qui n'est plus exactement celui qui se met en place aujourd'hui. Entre l'infrastructure et les utilisateurs, il importe en effet d'ajouter un autre actant : l'environnement informationnel de l'utilisateur qui s'est introduit comme un tiers entre la plateforme et l'individu pour devenir la principale cible du calcul numérique. Les interfaces numériques ne cessent de fabriquer des univers informationnels qui présentent et hiérarchisent l'information différemment en fonction des caractéristiques de l'utilisateur. Ce qui est visé est donc moins l'individu en tant que tel que la représentation numérique de l'environnement dans lequel il circule. Alors que les grandes infrastructures techniques ont souvent été dédiées à la production d'un espace organisé et régulé pour l'ensemble des bénéficiaires du service, les infrastructures numériques cherchent

\footnotetext{
$\overline{2}$ À côté de la variété et du volume, cette dimension de vélocité constitue le troisième "V $\mathrm{V}$ des discours tenus sur les big data. Voir, par exemple, Mayer-Schönberger et Cukier (2013).
} 
à reconfigurer l'espace de façon personnalisée pour chacun d'eux. La spécificité des infrastructures numériques auxquelles nous souhaitons prêter attention ici est de calculer des environnements différenciés plutôt que de produire un espace commun.

\section{Infrastructures de calcul}

Afin de donner un contour au genre d'environnement calculé visé dans ce texte, il est utile d'en donner d'abord quelques exemples sous forme stylisée. Les trois situations présentées ici se caractérisent par trois traits. En premier lieu, le calcul opéré au sein de l'infrastructure numérique personnalise ses opérations en fonction de chacun. En second lieu, cette personnalisation se sert de l'enregistrement du comportement de l'utilisateur par l'infrastructure numérique comme moyen pour vérifier la pertinence des prédictions qui lui ont été faites. Aussi est-il nécessaire de déployer une grille de saisie des activités de l'utilisateur qui soit intimement liée aux opérations statistiques effectuées par le calculateur afin de redessiner, en temps réel, l'environnement dans lequel de nouvelles actions sont possibles. Enfin, le dispositif exerce son contrôle sur l'environnement de l'utilisateur tout en préservant l'autonomie de ce dernier.

\section{Premier exemple}

Les algorithmes de Google, le PageRank, et de Facebook, le EdgeRank, ont été construits autour de principes, des règles (relativement) explicites enfermant un principe général, uniforme et intelligible. Le PageRank mesure l'autorité des sites en considérant que le fait d'avoir été cité par des pairs ayant eux-mêmes été beaucoup cités constitue un signe de reconnaissance (Cardon, 2012). Mobilisant un principe d'affinité, le EdgeRank donne une visibilité supérieure aux informations qui ont été publiées par les " amis » avec lesquels l'utilisateur a des interactions les plus fréquentes. Autorité et affinité, même si l'on peut discuter de la technique d'approximation qu'instaure un dispositif automatisé de calcul, constituent des principes qui enferment des valeurs identifiables et générales. Ces principes d'ordonnancement fonctionnent comme des règles explicites donnant au fonctionnement de l'algorithme une 
intelligibilité qui peut être partagée avec l'utilisateur. Même si ces principes sont toujours actifs dans le fonctionnement de ces deux algorithmes, le calcul qu'ils effectuent est cependant de plus en plus commandé par des méthodes de personnalisation qui peuvent altérer le principe initial en fonction des traces comportementales des internautes (Granka, 2010). De plus en plus, l'information qui enrichit l'algorithme $\mathrm{du}$ moteur de recherche consiste à savoir sur quel lien l'utilisateur a cliqué afin de réviser l'ordre des classements qui lui sont proposés. Si, sur Google, l'internaute ne clique pas sur les premiers liens d'autorité que lui propose le service, mais sur des liens plutôt commerciaux, universitaires ou institutionnels, l'algorithme va progressivement amender son classement afin de donner à l'internaute des listes de sites qui correspondent à ses clics réguliers. Si, sur Facebook, l'utilisateur ne clique pas sur les informations publiées par ses amis, mais sur les liens très populaires qui ont été poussés dans son newsfeed par la célérité de leur diffusion virale, l'algorithme va donner plus d'importance à ces informations qui n'ont pas été portées dans son fil d'actualité par les amis avec lesquels il a une affinité forte, mais par des amis à faible affinité (Gerlitz et Helmond, 2013). Désormais, l'environnement numérique dans lequel interagit l'utilisateur est de plus en plus construit, de façon ad hoc, à partir de la trace calculée de ses actions antécédentes.

\section{Deuxième exemple}

Dans le domaine du marketing numérique, le marché publicitaire "programmatique ", qui représente approximativement $20 \%$ du marché de la publicité en ligne, est désormais confié aux automates de real time bidding (RTB) des ad exchanges - technique aussi appelée "publicité programmatique ». L'opération de calcul ne dure pas plus des deux cents millisecondes qui séparent le clic appelant une page web et l'affichage de la bannière publicitaire sur l'écran de l'utilisateur. Pendant cette infime durée, le cookie de l'utilisateur déposé dans son navigateur par un data provider (Exelate, Bluekai, Weborama...) envoie ses traces de navigation passée à un système d'enchère automatique qui permet aux annonceurs en compétition de se battre pour proposer un prix d'affichage de leur bandeau publicitaire (Beauvisage et Mellet, 2016). Les annonceurs estiment à partir de leur propre base de données de comportements et de profils d'autres utilisateurs, les chances statistiques de voir cet 
utilisateur cliquer sur telle ou telle bannière publicitaire afin de fixer un prix d'enchère. Le gagnant pourra ensuite afficher sa publicité sur la page que l'utilisateur est en train de charger. Lors de cette opération, les annonceurs privilégient de plus en plus les traces de comportements de navigation des utilisateurs, plutôt qu'une connaissance client sous la forme de catégories (sexe, âge, niveau de revenu, segment marketing), informations dont ils disposent rarement à travers les cookies. Le choix de l'environnement publicitaire qui va être poussé vers l'utilisateur est ainsi confectionné en temps réel à partir d'une analyse comportementale des traces de ses actions passées.

\section{Troisième exemple}

Certains dispositifs mis en place par le secteur de l'assurance automobile proposent un service ajustant la prime au nombre de kilomètre parcouru (" pay as you drive »). Profitant du développement des objets connectés, les assureurs expérimentent désormais un service appelé " pay how you drive ». Il ne s'agit plus d'indexer la tarification à une catégorie homogène et continue, le nombre de kilomètres effectué indépendamment de la manière dont ils ont été parcourus, mais au comportement du conducteur : vitesse de conduite, usage des freins, rythme des accélérations, etc. Même si ces dispositifs restent encore expérimentaux, les offreurs de service se préparent à réaliser un couplage de plus en plus fin entre les données de conduites individuelles et un ensemble de données géolocalisées relatives à l'environnement de la conduite (cartographie, état des routes, du trafic, météo, etc.). La solution tarifaire proposée à l'utilisateur devient ainsi une conséquence modulable de ses comportements enregistrés de façon continue et précise par les nouvelles infrastructures numériques.

Ces situations renvoient à des contextes très variés, mais elles présentent une propriété commune. Ces dispositifs ne cherchent pas à contraindre l'utilisateur en lui imposant un calcul qui viendrait de l'extérieur lui imposer un programme qui aurait été décidé par d'autres. Ils prétendent calculer l'utilisateur, en quelque sorte, de l'intérieur de sa propre conduite. Les calculs qui servent à fabriquer l'environnement guidant l'utilisateur prennent forme depuis les traces du choix de ses actions passées. Il y a quelque chose de singulier dans cette manière de lier intimement l'autonomie de l'individu à la fabrication d'un 
environnement façonné par ses choix. Deux traits peuvent caractériser ce type d'infrastructure de calcul. Le premier tient à l'internalisation de la production de la règle de calcul dans les données elles-mêmes - ce qui est en jeu, tendanciellement, dans ce processus est la minimisation $\mathrm{du}$ poids des hypothèses ex ante au profit d'un réglage ex post des recommandations en fonction des données comportementales. Un tel processus contribue à faire des données, et donc de l'infrastructure d'enregistrement qui les rend possibles, le support même des calculs. Le second est que ce mécanisme d'internalisation peut se lire de deux façons. Une première lecture, politique, souligne le fait que, dans une logique néolibérale, l'individu est rendu responsable des choix qui le calculent. Une seconde lecture, attachée à la transformation des régimes de calculabilité, s'attache au fait que l'horizon visé par ces dispositifs est une sorte d'inductivisme radical couplant directement la production des choix à venir des individus au calcul sans hypothèse de leurs choix passés. C'est en s'efforçant de lier ensemble ces deux types de lecture que l'on voudrait caractériser la forme de ces nouvelles infrastructures de calcul des conduites.

\section{Gouverner l'environnement numérique de l'utilisateur}

Beaucoup de travaux scrutent la manière dont les grands acteurs du Web mettent en œuvre des mécanismes de contrôle, de manipulation et d'assujettissement des internautes. Ces critiques rendent cependant mal compte de l'expérience concrète des utilisateurs qui, s'ils partagent ces craintes, ne ressentent pas en revanche les effets de mécanismes de contrôle ou de limitation dans leurs propres usages. Le décalage qui s'accuse entre les représentations et les pratiques des internautes est particulièrement évident dans le cas du paradoxe de la privacy qui voit l'augmentation des représentations négatives face à la surveillance numérique n'avoir aucun effet limitant sur les pratiques d'exposition de soi en ligne (Kaplan, 2010)3. Aussi l'analyse du type de pouvoir qui s'exerce au sein des plateformes numériques doit-elle parvenir

Pour une interprétation soulignant le fait que le " paradoxe de la vie privée " n'est pas un arbitrage " choisi " entre vie privée et utilité des services, mais une obligation contrainte et résignée à céder ses données aux infrastructures numériques, voir Turow et al. (2015). 
à penser ensemble l'autonomie des individus et le contrôle de leur environnement ${ }^{4}$. "Comment ne pas être trop gouvernés ? " interrogeait Michel Foucault dans les cours qu'il a donnés au Collège de France entre 1977 et 1979 (Foucault, 2004a et b). Le type de pouvoir qui s'exerce au sein de la gouvernementalité néolibérale, suggère-t-il, ne se laisse pas décrire dans le vocabulaire du couple contrôle/discipline, mais dans un autre format qu'il faudrait appeler environnement/utilité. "Plutôt que de demander à des sujets idéaux ce qu'ils ont pu céder d'eux-mêmes ou de leurs pouvoirs pour se laisser assujettir ", invite Michel Foucault, " il faut chercher comment les relations d'assujettissement peuvent fabriquer des sujets " (Foucault, 2004a, p. 239). Le type de " conduite des conduites " qu'instaurent les nouvelles infrastructures numériques a besoin de l'autonomie des internautes. Celles-ci ne cessent en effet d'encourager et d'intensifier chez les individus la liberté de choix et d'expression. Les plateformes numériques se présentent comme un espace de procédures agnostiques dans lequel les internautes sont libres de choisir les formes et le contenu de leurs activités. Elles épousent étroitement les dynamiques d'individuation de nos sociétés en proposant un nouveau contrat social qui déplace, court-circuite et érode les mécanismes de confiance et de loyauté adressés à la régulation de l'État, aux formes marchandes de l'économie industrielle et, plus généralement, aux techniques de représentation traditionnelles (médias, associations, syndicats, partis, etc.). De plus en plus - et avec une adhésion qui ne se laisse pas desceller par la connaissance des risques pris dans ce nouveau pacte, notamment en termes de vie privée -, l'expressive "société des individus " déplie de nouvelles capacités d'agir dans l'architecture des plateformes des GAFA (Google, Apple, Facebook, Amazon). L'ouverture et la tolérance accordée par les plateformes à la diversité des choix, même les plus minoritaires, des internautes invitent donc à déplacer le regard vers une autre cible sur laquelle elles exercent leur pouvoir en intervenant " non pas sur les joueurs du jeu, mais sur les règles du jeu ». Cette manière de conduire les conduites, "qui ne serait pas du type de l'assujettissement interne des individus, mais une intervention de type environnemental " (Foucault, 2004b, p. 265), offre une perspective très éclairante pour

Sur l'impossibilité d'utiliser le modèle coercitif de la domination et de la contrainte pour décrire les expériences expressives, multiples, curieuses et diverses des internautes, voir Harcourt (2015). 
analyser les nouvelles infrastructures numériques. Si ces dernières refusent de contraindre les internautes, elles fabriquent en revanche l'environnement informationnel dans lequel ils circulent en agençant une gamme variée d'artefacts destinés à lister, guider, recommander, géolocaliser ou notifier les opportunités qui leur sont offertes. C'est à travers la production calculée d'une "architecture de choix ", un espace d'opportunités permettant à chacun d'explorer de nouvelles possibilités d'activités sans que celles-ci ne semblent prescrites ou commandées, que se manifeste " cette intervention de type environnemental ". Le développement des politiques de "paternalisme libertaire " du nudge en constitue une illustration exemplaire (Sunstein, 2014). Les dispositifs de " coup de pouce " guident sans imposer et orientent sans contraindre. Ils déplacent vers l'environnement des contraintes que l'on ne souhaite pas appliquer directement aux personnes sous la forme d'interdits, de règles ou de disciplines. Les techniques calculatoires des mondes numériques déploient clairement leurs ambitions dans cette perspective : architecturer l'environnement des choix des internautes sans pour autant leur imposer la discipline d'une contrainte directe.

Comme le soulignait Michel Foucault, ce qui caractérise cette forme de gouvernementalité par l'environnement, c'est que le point de contact entre les motivations des individus et l'architecture dans laquelle ils les déploient est un simple rapport d'utilité. C'est ce nœud utilitariste qui permet aux plateformes d'attacher le désir d'autonomie des individus à leurs dispositifs procéduraux. Utilité économique pour les architectes des environnements numériques, mais aussi utilité pour les praticiens des services rendus par les plateformes (personnalisation des informations, repérage cognitif, praticité, réduction des prix, simplification des transactions, etc.). Ce qui attache les usagers aux plateformes qui les exploitent sans les aliéner, c'est que le service qu' elles rendent est utile et que les utilisateurs ne semblent guère disposés à négocier ou sacrifier cette utilité . Aussi les industries numériques cherchent-elles sans doute moins à domestiquer, contrôler ou formater leurs utilisateurs qu’à orchestrer

Dans les enquêtes, ceux qui s'inquiètent le plus de la personnalisation algorithmique sont aussi ceux dont la vie, de mille et une manières, est soutenue par des activités de services, des exigences et des habitudes qui règlent "par défaut " l'environnement sur leur style de vie. Comme le souligne Cass Sunstein, il existe une inégalité sociale peu perçue entre ceux dont la vie est soumise à l'exigence continue de faire de " petits choix " de subsistance et ceux qui ont suffisamment de ressources pour 
les nouvelles utilités qui ne cessent d'apparaittre avec l'intensification et l'individualisation des formes de la vie sociale : accélération temporelle et désynchronisation des rythmes sociaux, multiplication des activités, enrichissement des expériences, accroissement de la mobilité, diversification des cycles de vie, etc. L'augmentation continue des interdépendances sociales et des opportunités d'expérience de vie constitue sans doute la racine la plus profonde de l'adhérence que les plateformes exercent sur les formes contemporaines de la vie sociale. Mais c'est aussi cet impératif d'efficacité visant à maximiser la satisfaction des utilisateurs qui conduit les techniques statistiques destinées à calculer l'environnement de l'utilisateur à prendre appui de façon toujours plus pressante sur l'enregistrement de ses comportements.

\section{Calculer la société par les comportements}

Les techniques de calcul mobilisées dans les nouvelles infrastructures numériques font en effet apparaitre une transformation du « style de raisonnement statistique " ${ }^{6}$ qui épouse les singularités de cette forme de conduite des conduites. Il est difficile de réduire à une seule direction les multiples déploiements des méthodes algorithmiques contemporaines. Cependant, il ne fait pas de doute quel'une des principales caractéristiques des formes calculatoires des big data est la généralisation des techniques d'apprentissage (machine learning). Comme dans notre premier exemple où les clics de l'internaute contribuent à personnaliser le calcul de ses prochaines requêtes, les modèles apprenants révisent les règles du calcul à partir de l'évaluation de l'efficacité de leurs prédictions qu'ils mesurent à travers les traces comportementales de l'utilisateur. Si les techniques d'apprentissage ont une histoire ancienne ${ }^{7}$, leur développement contemporain appelle une rapide caractérisation afin de faire apparaître le déplacement qu'elles opèrent à l'égard des formes traditionnelles de la statistique sociale. Les calculateurs des infrastructures numériques

personnaliser "par défaut " leur environnement afin de se réserver le privilège libéral de faire de "grands choix ». Voir Sunstein (2015).

6 Sur l'usage de cette notion par Ian Hacking pour caractériser l'émergence du paradigme probabiliste, voir Hacking (1990, p. 6).

Sur l'histoire de la statistique "bayésienne " dans son opposition au modèle "fréquentiste », voir McGrayne (2011). 
proposent en effet de calculer la société d'une manière qui ne correspond plus aux exigences de centralité, d'univocité et de généralité des méthodes statistiques distribuant les individus autour de la moyenne selon une loi normale. Si les mesures d'audience ordonnées autour de l'idée de popularité et le classement des moteurs de recherche (lorsque la personnalisation n'y est pas prépondérante) organisé autour de la valeur d'autorité répondent à ces exigences classiques, les nouvelles métriques de réputation des réseaux sociaux produisent une valeur différente selon la position dans le réseau de ceux qui, avec une sensibilité différente, sont exposés à ses effets. Plus encore, les techniques de prédiction personnalisée affichent une rupture franche avec les statistiques traditionnelles. Elles ne cherchent plus à construire une mesure centrale, mais à mesurer depuis l'individu. Elles ne fabriquent pas une représentation univoque, mais la modifient selon la position de chacun ${ }^{8}$. Elles ne visent pas une généralité commune à l'ensemble des individus statistiques, mais anticipent des vérités locales (Mackenzie, 2013).

Comme l'a montré l'histoire de la statistique, le déploiement de vastes dispositifs de mesure de la société a accompagné l'idée probabiliste que si les phénomènes sociaux n'étaient pas régis par des lois déterministes, il était cependant possible d'interpréter la société à partir de régularités observables (Porter, 1986 ; Gigerenzer et al., 1989). Comme le souligne Ian Hacking, le développement des statistiques est inséparable de l'existence de sociétés démocratiques et libérales où la reconnaissance du principe d'indétermination des actions individuelles est, en quelque sorte, récupérée par le haut, à travers l'objectivation de régularités globales (Hacking, 2002). Le paradigme probabiliste est ainsi venu substituer à la causalité des lois naturelles une technique de réduction de l'incertitude qui, au terme d'une vaste entreprise de catégorisation des populations, a permis de distribuer les comportements selon une loi normale. Aussi l'immense travail destiné à enregistrer, quantifier et mesurer la société qui s'est mis en place au XIX $\mathrm{X}^{\mathrm{e}}$ siècle a-t-il permis à la statistique sociale d'établir la confiance dans les nombres (Porter, 1995). La statistique «fréquentiste » de «l'homme moyen » de Quetelet généralise l'idée que les phénomènes sociaux peuvent être corrélés à des

8 Sur cette typologie de quatre familles de calcul algorithmique valorisant tour à tour la popularité, l'autorité, la réputation et la prédiction personnalisée, voir Cardon (2015). 
causes constantes. "Une distribution de forme normale de mesures réparties autour d'une valeur modale permet ainsi d'inférer l'existence d'une cause constante, ou d'un ensemble de causes dont la combinaison produit des effets constants pendant la série de ces observations ", explique Alain Desrosières. "Cette forme permet de construire une classe d'équivalence entre ces événements, liés entre eux par le fait qu'ils sont déterminés en partie par une cause commune à tous, mais qu'une autre partie de ces déterminations relève de causes accidentelles différentes pour chacun d'entre eux " (Desrosières, 2000). Aussi est-ce l'existence d'une distribution en loi normale qui permet, en dépit des " causes accidentelles ", de faire des " causes constantes " le socle fiable des variables explicatives qui vont nourrir la production d'une connaissance de la société par la démographie ou la sociologie. De façon à la fois pratique, à travers l'investissement et l'entretien d'un système codifié d'enregistrements réguliers, et épistémologique, en distribuant les événements statistiques autour de valeurs moyennes, la méthode " fréquentiste " de la statistique sociale a ainsi contribué à solidifier des " causes constantes". Encastrées dans des dispositifs institutionnels et techniques réguliers et fiables, elles ont acquis une sorte d'extériorité qui en font un appui solide pour établir des corrélations avec de nombreux phénomènes sociaux et en inférer une interprétation causaliste. La chosification des combinaisons de causes constantes qui a permis de dresser des tableaux de plus en plus extensifs de la société est ainsi très intimement liée à la production méticuleuse de conventions catégorielles permettant d'enregistrer des distributions d'événements statistiques selon une loi normale. Or c'est un modèle différent, celui de la probabilité des causes, que les techniques dites "bayésiennes " longtemps refoulées dans la pensée statistique proposent de rouvrir en introduisant la possibilité que des "causes accidentelles " qui ne sont pas distribuées selon une loi normale puissent devenir le support de corrélations statistiques d'un autre genre.

Si l'on voulait caractériser succinctement le tournant pris par ce nouveau style de raisonnement statistique, on pourrait dire qu'il substitue la matrice creuse à la loi normale. Dans les environnements numériques, la multiplication des enregistrements contribue à une augmentation considérable du nombre des variables disponibles au calcul. Même si les matrices qui les recueillent sont souvent « creuses ", les calculs mis 
en ouvre n'abandonnent pas l'idée que, dans certains contextes, des variables rares et improbables puissent avoir un effet sur certains types de corrélations. Renouant avec les techniques inductives de l'analyse des données, les calculateurs numériques ne cherchent pas à réduire et à stabiliser l'espace des variables pertinentes. Les causes deviennent inconstantes et sont associées par le calculateur dans des assemblages changeants en fonction des objectifs locaux requis par la prédiction de l'environnement des différents utilisateurs. Trois traits typiques de la fabrication de ces nouveaux calculs peuvent être soulignés (Shmueli, 2010). Le premier est l'obsession de " descendre " sous les catégories trop globales pour chercher à enregistrer les données au niveau le plus fin et le plus élémentaire possible. Les techniques de calcul des big data font l'hypothèse qu'il est possible de produire de meilleures prédictions en s'affranchissant du périmètre balisé des variables qui fournissent des causes constantes et que, en augmentant le nombre de signaux par l'ajout de variables irrégulières ou rares, il serait possible d'augmenter la qualité des prédictions lorsqu'elles s'adressent à un individu statistique en particulier. C'est la raison pour laquelle un flux d'enregistrement de comportements est préféré à une identification catégorielle générique comme dans notre deuxième exemple de la publicité programmatique en real time bidding. À la différence de la mesure des opinions ou des expressions subjectives sur les réseaux sociaux, les infrastructures de calcul orientées vers la prédiction personnalisée s'intéressent principalement à des traces de conduites : cliquer, acheter, lire, se déplacer, etc. Souvent défendue par des chercheurs issus de la physique, l'idée que les enregistrements numériques pourraient nourrir une nouvelle sociologie quantitative est d'inspiration principalement behavioriste'. Le second trait de ce style de raisonnement statistique est le remplacement des modèles ex ante comportant des règles symboliques fixes au profit de modèles mouvants et distribués tels qu'ils sont mis en place dans les techniques d'apprentissage des forêts aléatoires, des supports vector machine ou des réseaux connexionniste (dits " de neurones ") ${ }^{10}$. La substitution progressive d'algorithmes à règles symboliques par des techniques massivement statistiques mettant en compétition une

$9 \quad$ Par exemple, dans cet état d'esprit : Pentland (2014).

10 Pour une présentation pédagogique des différentes techniques d'apprentissage, voir Domingos (2015). 
multiplicité de modèles construits et testés à la volée accompagne aussi dans les techniques d'apprentissage un principe inductif de " rasoir d'Occam " visant à diminuer systématiquement et autant que faire se peut les modélisations préalables (Shmueli, 2010). Ce faisant, les modèles algorithmiques deviennent opaques et ininterprétables par un humain. Le calcul ne cherche plus à comprendre ou à expliquer, mais à prédire efficacement. Un troisième trait de ces calculs tient à la généralisation du raisonnement bayésien de la probabilité des causes : ce qui, constantes et modèles, est défini a priori doit pouvoir être révisé par l'observation d'événements postérieurs. Le raisonnement bayésien permet la variabilité des causes en remontant " des effets vers une évaluation du degré de certitude des causes de ceux-ci " (Desrosières, 2000, p. 100). Cette variabilité est rendue possible par l'assouplissement des modèles et par l'hétérogénéité des jeux de données permettant de mettre en concurrence des gammes de corrélations entre les données qui varient selon les contextes. Dans notre troisième exemple, l'indexation de la prime d'assurance au nombre de kilomètres parcourus propose classiquement de faire une corrélation uniforme et stable distribuant de la même façon la valeur de la prime par conducteur en fonction d'une variable ayant la même consistance pour tous : le kilométrage. En revanche, lorsque la voiture enregistre des traces comportementales indiquant la manière dont le conducteur a effectivement parcouru ces kilomètres, les variables pouvant inférer la qualité d'une bonne conduite deviennent soudainement plus nombreuses, plus diverses et sont distribuées de façon très hétérogène. Elles sont soumises à des variations contextuelles extrêmement fortes. Ne pouvant être modélisées a priori, ce n'est que sous forme d'apprentissage qu'il va être possible de « choisir " le meilleur couplage entre le type de conduite de ce conducteur particulier et le calcul de risque d'accident ou d'endommagement de son véhicule. Les variables catégorielles, dont la qualité première était de fabriquer un espace commun de mesure, sont l'objet d'une décomposition multifactorielle qui permet de préciser et d'affiner le résultat pour produire une connaissance qui n'est plus globale, mais distribuée en chaque point de l'espace statistique.

Ces transformations des formats calculatoires témoignent d'une modification de la relation logique entre les hypothèses et les données. L'indétermination de plus en plus forte de la structure des grandes bases 
de données, leur indécidabilité structurelle, invite à substituer l'idée de causes efficientes à celle de causes premières. En quelque sorte, c'est le devenir-inattendu des individus, lorsque leur comportement est enregistré dans des espaces de mesure qui multiplient les dimensions, qui pousse les nouvelles techniques de calcul à réclamer toujours plus d'enregistrements comportementaux. Il n'est pas encore bien établi que ces méthodes donnent des résultats pertinents, notamment parce qu'en réalité les données qu'elles doivent recueillir sont si nombreuses que les capteurs actuels manquent de pertinence, mais le développement de "predictive analytics " constitue l'horizon calculatoire du déploiement des nouvelles infrastructures numériques (Siegel, 2013).

\section{Conclusion}

La transformation du raisonnement statistique mis en œuvre par ces dispositifs permet de faire retour sur les caractéristiques matérielles des nouvelles emprises que l'infrastructure numérique étend sur le monde social. Si les traces de comportements deviennent le signal permettant de régler les algorithmes sur un principe d'efficacité, il est alors nécessaire de capturer toujours plus d'informations au plus près de l'utilisateur. Le développement de la connaissance et du pouvoir statistique au XIX ${ }^{e}$ siècle est inséparable d'un travail extensif d'enregistrement et de codification d'un "espace de communes mesures " (Desrosières, 2000, p. 131). À travers les questionnaires standardisés, les nomenclatures, la mise en échelles et les techniques d'échantillonnage, il s'agissait alors de " faire tenir " des catégories suffisamment stables pour devenir des « causes constantes » de la connaissance des comportements. La manière dont le calcul numérique relance actuellement ce processus d'extension de l'espace des réalités statistiques procède moins de l'abstraction catégorielle que d'une grille d'enregistrement comportementaliste toujours plus étendue. Le développement des objets connectés, le souci d'une capture et d'une transmission en temps réel des informations, la recentralisation des calculs accompagnent le projet de ne pas calculer les comportements depuis des catégories identifiant durablement les individus, mais depuis la trace continue et changeante de leurs comportements. 


\section{Références}

Beaude B. (2014), Les fins d'Internet, Paris, FYP Éditions.

Beauvisage T., Mellet K. (2016), « Datassets: assetizing and marketizing personal data ", 4S/EASST Conference, Barcelona.

Blum A. (2012), Tubes. A Journey to the Centre of the Internet, New York, HarperCollins.

Bratton B. (2015), The Stack. On Software and Sovereignty, Londres, The MIT Press.

Burton F. (2016), « Une histoire du spam. Le revers de la communauté en ligne ", Réseaux, vol. 4, n 197-198, p. 33-67.

Cardon D. (2012), "Dans l'esprit du PageRank. Une enquête sur l'algorithme de Google ", Réseaux, vol. 31, n 177, p. 63-95.

Cardon D. (2015), A quoi rêvent les algorithmes. Nos vies à l'heure des big data, Paris, Seuil, coll. "La république des idées".

Citton Y. (2016), " Notre inconscient numérique. Comment les infrastructures du Web transforment nos esprits ", Revue du crieur, n ${ }^{\circ} 4$, juin, p. 147-148.

DeNardis L. (2014), The Global War for Internet Governance, New Haven (Conn.), Yale University Press.

Desrosières A. (2000), La politique des grands nombres. Histoire de la raison statistique, Paris, La Découverte.

Domingos P. (2015), The Master Algorithm. How the question for the ultimate machine will remake our world, Londres, Penguin Random House UK.

Durham Peters J. (2015), The Marvellous Clouds. Towards a Philosophy of Elemental Media, Chicago (Ill.), University of Chicago Press.

Foucault M. (2004a), Sécurité, territoire, population. Cours au Collège de France. 1977-1978, Paris, Gallimard/Seuil.

Foucault M. (2004b), Naissance de la biopolitique. Cours au Collège de France, 1978-79, Paris, Gallimard/Seuil, coll. «Hautes études ».

Gerlitz C., Helmond A. (2013), "The Like Economy: Social Buttons and the Data-intensive Web ", New Media \& Society, vol. 15, n 8, p. 13661373. 
Gigerenzer G., Swijtnik Z., Porter T., Daston L., Beatty J, Krüger L. (1989), The Empire of Chance. How Probability Changed Science and Everyday Life, Cambridge, Cambridge University Press.

Granka L. A. (2010), "The Politics of Search: A Decade Retrospective ", The Information Society, $\mathrm{n}^{\circ}$ 26, p. 364-374.

Hacking I. (1990), The Taming of Chance, Cambridge, Cambridge University Press.

Hacking I. (2002), L'émergence de la probabilité, Paris, Seuil.

Harcourt B. (2015), Exposed. Desire and Disobedience in the Digital Age, Cambridge (Mass.), Harvard University Press.

Kaplan D. (2010), Informatique, libertés, identités, Paris, FYP.

Kitchin R. (2014), The Data Revolution. Big Data, Data Infrastructures \& Their Consequences, Londres, Sage.

Lessig L. (2006), Code. Version 2.0, New York, Basic Books.

Mackenzie A. (2013), " Programming Subjects in the Regime of Anticipation: Software Studies and Subjectivity ", Subjectivity, vol. 6, $\mathrm{n}^{\circ}$ 4, p. 391-405.

Maxwell R., Miller S. (2012), Greening the Media, Oxford, Oxford University Press.

Mayer-Schönberger V., Cukier K. (2013), Big data. A Revolution that Will Transform How We Live, Work and Think, Boston (Mass.), An Eamon Dolan Book.

McGrayne S. B. (2011), The Theory that would not Die. How Bayes rule cracked the enigma code, heated down russian submarine \& emerge triumphant from two centuries of controversy, New Haven (Conn.), Yale University Press.

Pentland A. (2014), Social Physics. How Good Ideas Spread. The Lessons from a New Science, New York, The Penguin Press.

Porter T. M. (1986), The Rise of Statistical Thinking 1820-1900, Princeton (NJ), Princeton University Press.

Porter T. M. (1995), Trust in Numbers. The Pursuit of Objectivity in Science and Public Life, Princeton (NJ), Princeton University Press. 
Rouvroy A. Berns T. (2010), «Le nouveau pouvoir statistique. Ou quand le contrôle s'exerce sur un réel normé, docile et sans événement car constitué de corps "numériques" ", Multitudes, vol. 1, n 40, p. 88-103.

Sadin E. (2015), La vie algorithmique. Critique de la raison numérique, Paris, L'Échappée.

Shmueli G. (2010), «To Explain or to Predict? », Statistical Science, vol. 25, $\mathrm{n}^{\circ} 3$, p. 289-310.

Siegel E. (2013), Predictive Analytics. The power to predict who will click, buy, lie or die, Hoboken (NJ), John Wiley \& Sons.

Sunstein C. (2014), Why Nudge? The Politics of Libertarian Paternalism, New Haven (Conn.), Yale University Press.

Sunstein C. (2015), Choosing Not to Choose. Understanding the Value of Choice, Oxford, Oxford University Press.

Turow J., Hennessy M., Draper N. (2015), The Tradeoff Fallacy. How Marketers Are Misrepresenting American Consumers and Opening Them up to Exploitation, Philadelphia (Penn.), University of Pennsylvania. 
www.peterlang.com 